\title{
International Union of Basic and Clinical Pharmacology. CXI. Pharmacology, Signaling, and Physiology of Metabotropic Glutamate Receptors
}

\author{
Karen J. Gregory and Cyril Goudet
}

Drug Discovery Biology, Monash Institute of Pharmaceutical Sciences and Department of Pharmacology, Monash University, Parkville, Victoria, Australia (K.J.G.) and Institut de Génomique Fonctionnelle (IGF), University of Montpellier, Centre National de la Recherche Scientifique (CNRS), Institut National de la Sante et de la Recherche Medicale (INSERM), Montpellier, France (C.G.)

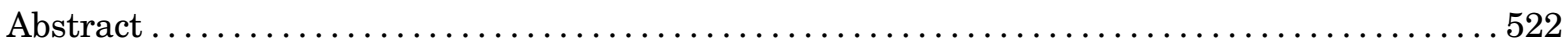

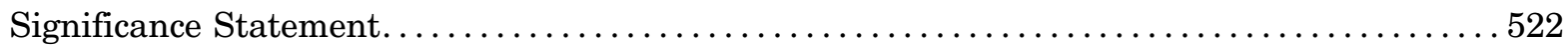

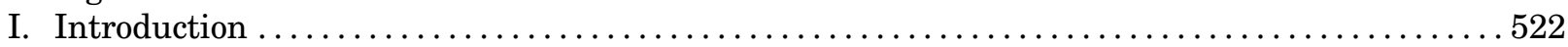

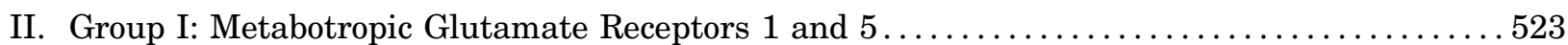

A. Receptor Subtypes and Splice Variants ................................ 523

B. Localization and Signal Transduction .................................... 524

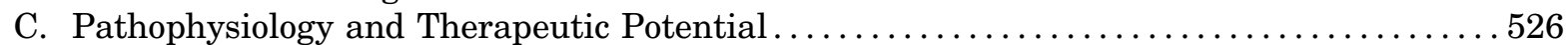

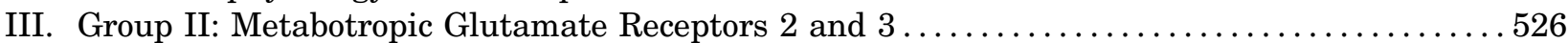

A. Receptor Subtypes and Splice Variants . . . . . . . . . . . . . . . . . . . . . . . . . 526

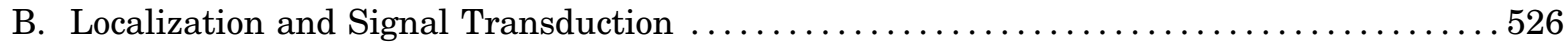

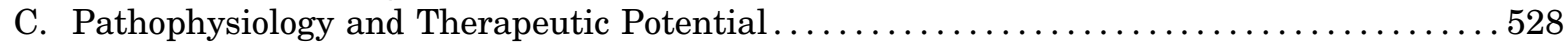

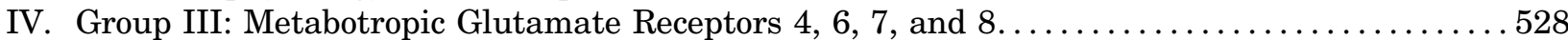

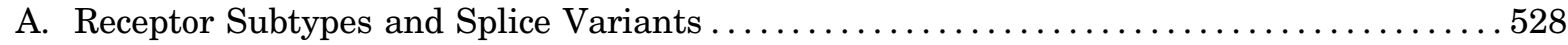

B. Localization and Signal Transduction ................................ 529

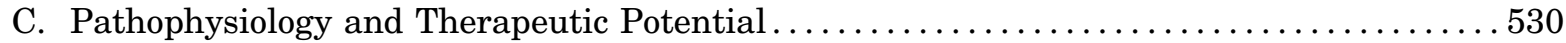

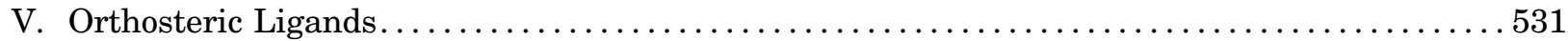

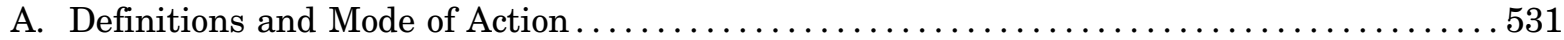

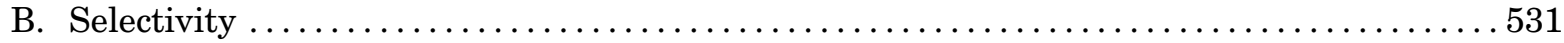

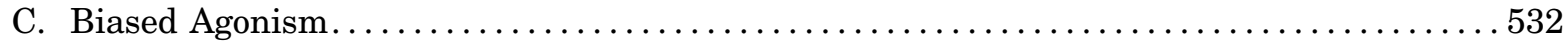

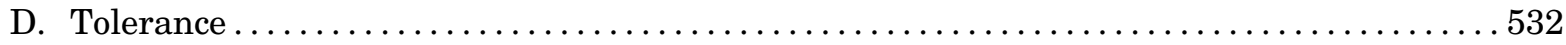

E. Orthosteric Ligands in the Clinic: Success and Failure ..................... 534

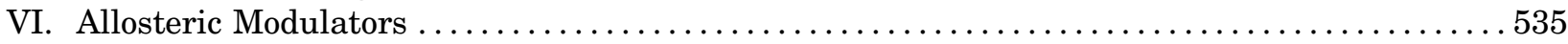

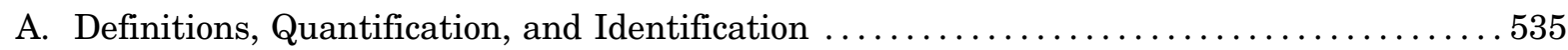

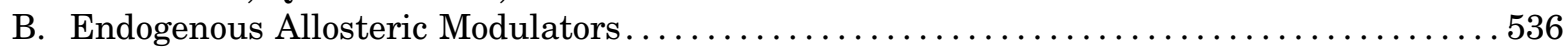

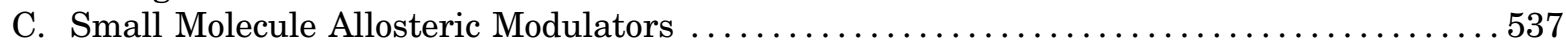

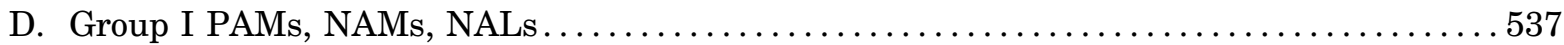

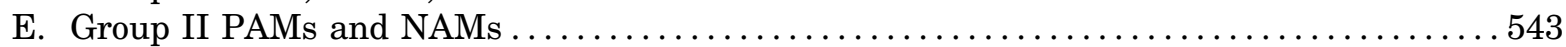

F. Group III PAMs and NAMs . . . . . . . . . . . . . . . . . . . . . . . . . . . . . . . 545

G. Allosteric Modulators Progressing to Clinical Trials. . . . . . . . . . . . . . . . . . . . . . . 546

H. Secondary Allosteric Sites within the VFT and 7TM Domains . . . . . . . . . . . . . . 546

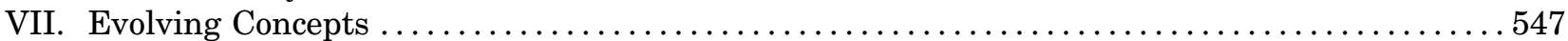

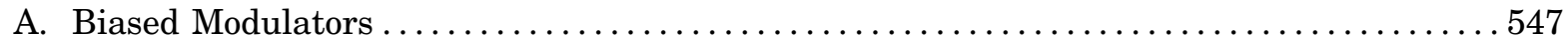

B. Location- and Context-Dependent Pharmacology ........................ 548

Address correspondence to: Karen J. Gregory, Drug Discovery Biology, Monash Institute of Pharmaceutical Sciences and Department of Pharmacology, Monash University, Parkville, Victoria, Australia. E-mail: karen.gregory@monash.edu; orCyril Goudet, Institut de Génomique Fonctionnelle, University of Montpellier, CNRS, INSERM, 141 rue de la cardonille, Montpellier 5, F34094, France. E-mail: cyril.goudet@ igf.cnrs.fr

C.G. was supported by grants from the Agence Nationale de la Recherche [Grants ANR-16-CE16-0010-01 and ANR-17-NEU3-0001-01 under the frame of Neuron Cofund]. K.J.G. was supported by Australian Research Council [Grant FT170100392] and National Health and Medical Research Council (Australia) [Grants APP1084775 and APP1123722].

https://doi.org/10.1124/pr.119.019133. 


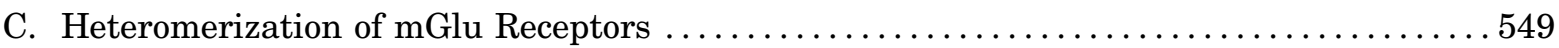

D. Optical Tools to Probe and Control mGlu Receptors.......................... 550

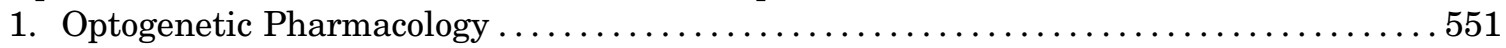

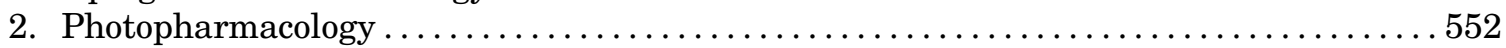

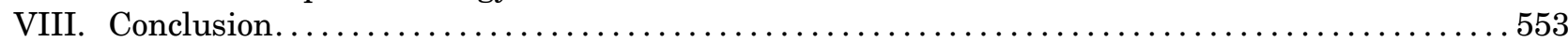

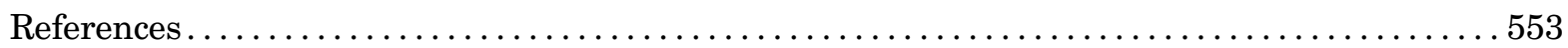

\begin{abstract}
Metabotropic glutamate (mGlu) receptors respond to glutamate, the major excitatory neurotransmitter in the mammalian brain, mediating a modulatory role that is critical for higher-order brain functions such as learning and memory. Since the first mGlu receptor was cloned in 1992, eight subtypes have been identified along with many isoforms and splice variants. The mGlu receptors are transmembranespanning proteins belonging to the class $C G$ protein-coupled receptor family and represent attractive targets for a multitude of central nervous system disorders. Concerted drug discovery efforts over the past three decades have yielded a wealth of pharmacological tools including subtype-selective agents that competitively block or mimic the actions of glutamate or act allosterically via distinct sites to enhance or inhibit receptor activity. Herein, we review the physiologic and pathophysiological roles for individual mGlu receptor subtypes including the pleiotropic nature of intracellular signal transduction arising from each. We provide a comprehensive analysis of the in vitro
\end{abstract}

and in vivo pharmacological properties of prototypical and commercially available orthosteric agonists and antagonists as well as allosteric modulators, including ligands that have entered clinical trials. Finally, we highlight emerging areas of research that hold promise to facilitate rational design of highly selective mGlu receptor-targeting therapeutics in the future.

Significance Statement-The metabotropic glutamate receptors are attractive therapeutic targets for a range of psychiatric and neurological disorders. Over the past three decades, intense discovery efforts have yielded diverse pharmacological tools acting either competitively or allosterically, which have enabled dissection of fundamental biological process modulated by metabotropic glutamate receptors and established proof of concept for many therapeutic indications. We review metabotropic glutamate receptor molecular pharmacology and highlight emerging areas that are offering new avenues to selectively modulate neurotransmission.

\section{Introduction}

Glutamate is the major excitatory neurotransmitter in the human brain mediating its effects via two distinct receptor classes. Ionotropic glutamate receptors are ligand-gated ion channels that rapidly cause membrane depolarization in response to glutamate. On the other hand, metabotropic glutamate (mGlu) receptors have a modulatory role exerted over a longer time scale including influencing neuronal excitability and synaptic plasticity as well as activity of nonneuronal cells.

The mGlu receptors are a family of eight class $\mathrm{C} \mathrm{G}$ protein-coupled receptors (Acher et al., 2019; Alexander et al., 2019). They comprise a large extracellular N-terminal domain where glutamate binds, termed the Venus flytrap (VFT) domain, link to 7 transmembrane (7TM) alpha-helical

ABBREVIATIONS: ADX71743, 6-(2,4-dimethylphenyl)-2-ethyl-4,5,6,7-tetrahydro-1,3-benzoxazol-4-one; Akt, RAC-alpha serine/threonineprotein kinase; AMN082, N,N'-dibenzhydrylethane-1,2-diamine dihydrochloride; AMPA, $\alpha$-amino-3-hydroxy-5-methyl-4-isoxazolepropionic acid; ATCM, allosteric ternary complex model; AZ12216052, 2-[[(4-bromophenyl)methyl]sulfanyl]-N-[4-(butan-2-yl)phenyl]acetamide; BINA, 4[3-[(2-cyclopentyl-6,7-dimethyl-1-oxo-2,3-dihydroinden-5-yl)oxymethyl]phenyl]benzoic acid; CDPPB, 3-cyano- $N$-(1,3-diphenyl-1H-pyrazol5-yl)benzamide; CNS, central nervous system; CPCCOEt, ethyl (7Z)-7-hydroxyimino-1,7a-dihydrocyclopropa[b]chromene-1a-carboxylate; CPPHA, $N$-[4-chloro-2-(phthalimidomethyl)phenyl]salicylamide; CRD, cysteine-rich domain; DCG-IV, (2S,2'R,3'R)-2-(2',3'-dicarboxycyclopropyl) glycine; DHPG, dihydroxyphenylglycine; DPFE, 1-(4-(2,4-difluorophenyl)piperazin-1-yl)-2-((4-fluorobenzyl)oxy)ethan-1-one; EAAT, excitatory amino acid transporter; ERK1/2, extracellular signal-regulated kinases 1 and 2; GIRK, G protein-coupled inwardly rectifying potassium channel; GPCR, G protein-coupled receptor; GSK, glycogen synthase kinase; $\mathrm{iCa}^{2+}$, intracellular $\mathrm{Ca}^{2+} ; \mathrm{IP}_{1}$, inositol monophosphate; $\mathrm{K}_{\mathrm{B}}$, equilibrium dissociation constant; L-AP4, L-(+)-2-amino-4-phosphonobutyric acid; LID, levodopa-induced dyskinesia; LSP4-2022, (2S)-2-amino-4(\{[4-(carboxymethoxy)phenyl](hydroxy)methyl\}(hydroxy)phosphoryl)butanoic acid; LTD, long-term depression; LY354740, (1S,2S,5R,6S)-2amino-bicyclo[3.1.0] hexane-2,6-dicarboxylic acid; LY379268, (1R,4R,5S,6R)-4-amino-2-oxabicyclo[3.1.0]hexane-4,6-dicarboxylic acid; LY487379, 2,2,2-trifluoro- $N$-[4-(2-methoxyphenoxy)phenyl]- $N$-(3-pyridinylmethyl)-ethanesulfonamide; mGlu, metabotropic glutamate; M-5MPEP, 2-[2(3-methoxyphenyl)ethynyl]-5-methylpyridine; ML337, [2-fluoro-4-[2-(4-methoxyphenyl)ethynyl]phenyl][(3R)-3-hydroxy-1-piperidinyl]methanone; MMPIP, 6-(4-methoxyphenyl)-5-methyl-3-(4-pyridinyl)-isoxazolo[4,5-c]pyridin-4(5H)-one; MNI-137, 4-(8-bromo-5-oxo-3,4,5,6-tetrahydro-1,6-benzodiazocin-2-yl)pyridine-2-carbonitrile; MPEP, 2-methyl-6-(phenylethynyl)-pyridine; MTEP, 3-((2-methyl-4-thiazolyl)ethynyl) pyridine; NAL, neutral allosteric ligand; NAM, negative allosteric modulator; NMDA, $N$-methyl-D-aspartate; PAM, positive allosteric modulator; PD, Parkinson's disease; PHCCC, $N$-phenyl-7-(hydroxyimino)cyclopropa[b]chromen-1a-carboxamide; PI3K, phosphoinositide-3-kinase; PKC, protein kinase C; PLC, phospholipase C; PORTL, photoswitchable orthogonal remotely tethered ligands; RO 67-7476, 2-(4-fluorophenyl)-1-(4-methylphenyl)sulfonylpyrrolidine; 7TM, 7 transmembrane; VFT, Venus flytrap; VU0155041, (1R,2S)-2-[(3,5-dichlorophenyl) carbamoyl]cyclohexane-1-carboxylic acid; VU0360172, N-cyclobutyl-6-[2-(3-fluorophenyl)ethynyl]-3-pyridinecarboxamide hydrochloride; VU0409551, [6,7-dihydro-2-(phenoxymethyl)oxazolo[5,4-c]pyridin-5(4H)-yl](fluorophenyl)methanone; VU0422288, $N$-[3-chloro-4-[(5-chloro-2pyridinyl)oxy]phenyl]-2-pyridinecarboxamide; VU0483605, 3-chloro-N-[3-chloro-4-(4-chloro-1,3-dihydro-1,3-dioxo-2H-isoindol-2-yl)phenyl]-2pyridinecarboxamide; VU29, $N$-(1,3-diphenyl-1H-pyrazolo-5-yl)-4-nitrobenzamide; XAP044, 7-hydroxy-3-(4-iodophenoxy)-4H-chromen-4-one; $5-\mathrm{HT}_{2 \mathrm{~A}}$, serotonin receptors $2 \mathrm{~A}$. 
domains via a cysteine-rich domain (CRD) (Fig. 1). The mGlu receptors are obligate dimers mediated by an interprotomer disulfide bond at the top of the VFT domains. Structural studies indicate that the bilobed VFT domains adopt a closed conformation upon agonist binding (Kunishima et al., 2000; Tsuchiya et al., 2002; Muto et al., 2007; Monn et al., 2015a,b; Koehl et al., 2019). The CRD transmits the active VFT conformation to the 7TM via interactions with the second extracellular loop of the 7TM (Koehl et al., 2019). When activated the 7TM domains come into closer proximity, with transmembrane domain 6 mediating dimerization between the 7TM domains of the two protomers (El Moustaine et al., 2012; Doumazane et al., 2013; Xue et al., 2015; Koehl et al., 2019; reviewed in Pin and Bettler, 2016). Ultimately, the active 7TM domains couple to intracellular transducers to elicit a cellular response.

The eight mGlu receptor subtypes are commonly divided into three groups based on sequence identity,

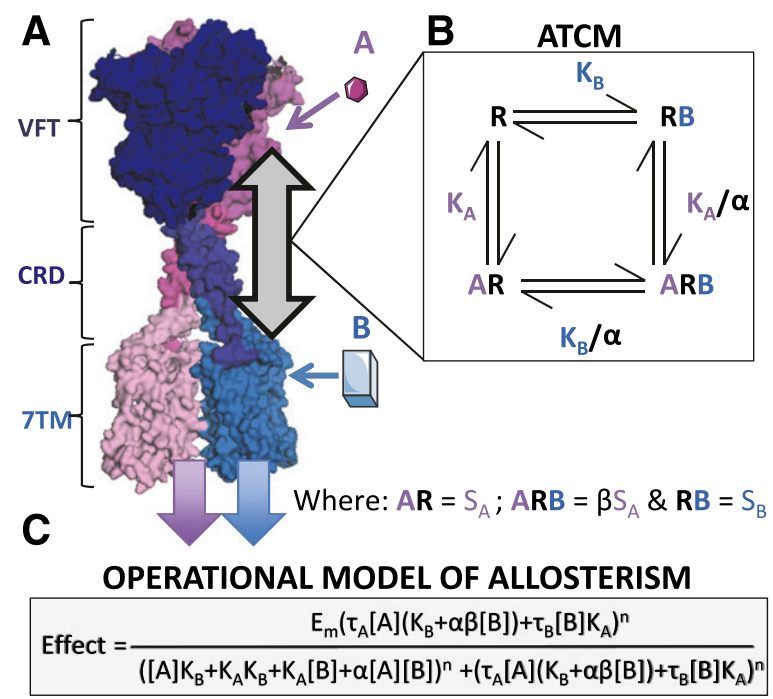

Fig. 1. Dimeric structure of full-length mGlu receptors and the relationships between different binding pockets. (Panel A) Metabotropic glutamate receptors are constitutive dimers mediated by extensive interactions between the VFT domains including an interprotomer disulfide bond at the top of the VFTs. In this surface representation of full-length mGlu $_{5}$ structure (Protein Data Bank identifier: 6N51) (Koehl et al., 2019), the two protomers are colored blue and purple, with the three major domains in different shades. The orthosteric agonist (A), glutamate, binds in the cleft between the two lobes of the VFT. When the VFT is bound to agonist, the CRD and 7TM become closer in proximity. The majority of small molecule allosteric modulators (B) are thought to interact with the 7TM. (Panel B) The simultaneous binding of an allosteric modulator and orthosteric agonist to the receptor can alter the affinity of the receptor for each ligand in a reciprocal fashion. The simplest model to describe this interaction and quantify the cooperativity $(\alpha)$ between these sites is the ATCM. (Panel C) Allosteric ligands can modulate receptor activity in response to orthosteric agonist $(\beta)$ or act as agonists (positive or inverse). Such that the stimulus $\left(\mathrm{S}_{\mathrm{A}}\right)$ arising from the agonist occupied receptor (AR) is different when simultaneously occupied by an allosteric modulator (ARB) and the allosteric modulator bound receptor $(\mathrm{RB})$ can engender a distinct response $\left(\mathrm{S}_{\mathrm{B}}\right)$. To account for functional effects, the most commonly applied framework is the operational model of allosterism (Leach et al., 2007; Gregory et al., 2012), where the ATCM has been incorporated into an operational model of agonism (Black and Leff, 1983).
G protein coupling preferences, and pharmacology. In addition to forming constitutive homodimers, heteromers have been observed among group I members and between group II and III subtypes (Doumazane et al., 2011). The various mGlu receptor subtypes are ubiquitously expressed throughout the brain in neurons and glia, with the exception of $\mathrm{mGlu}_{6}$ receptor, for which expression is restricted to the retina (reviewed in Ferraguti and Shigemoto, 2006). Peripheral mGlu receptors (reviewed in detail by Julio-Pieper et al., 2011) are found in tissues that receive glutamatergic innervation (e.g., heart, gastrointestinal tract, pain circuitry; Pereira and Goudet, 2019) but are also present in some nonexcitatory tissues and organs (e.g., immune cells, liver, kidney). Herein we provide a brief overview of the fundamental biology of the different mGlu subtypes and intracellular signaling, followed by an in-depth discussion of pharmacological agents and therapeutic indications with a focus on central nervous system (CNS) disorders.

\section{Group I: Metabotropic Glutamate Receptors 1 and 5}

\section{A. Receptor Subtypes and Splice Variants}

The group I mGlu receptors include $\mathrm{mGlu}_{1}$ and $\mathrm{mGlu}_{5}$. The $\mathrm{mGlu}_{1}$ receptor gene (GRM1) and its first three splice variants were cloned in rat in 1992 (Pin et al., 1992; Tanabe et al., 1992). In humans, there are seven $\mathrm{mGlu}_{1}$ splice variants $(\mathrm{a}, \mathrm{b}, \mathrm{d}, \mathrm{f}, \mathrm{g}, \mathrm{h})$ that differ in $\mathrm{C}$ terminus length (Sugiyama et al., 1987; Tanabe et al., 1992; Laurie et al., 1996; Makoff et al., 1997; Soloviev et al., 1999; DiRaddo et al., 2013) [Ensembl gene identifier: ENSG00000152822]. In addition, 12 single nucleotide polymorphisms within the GRM1 coding region have been identified in patients with schizophrenia (Frank et al., 2011; Ayoub et al., 2012), suggesting $\mathrm{mGlu}_{1}$ may be a viable therapeutic target for psychosis (Cho et al., 2014b). Spontaneous mutations in GRM1 are also associated with ataxia (Watson et al., 2017). The mGlu $_{5}$ receptor is encoded by the GRM5 gene [ENSG00000168959], localized in human chromosome 11, and was first cloned in rat in 1992 (Abe et al., 1992) and in human in 1994 (Minakami et al., 1994). Alternative splicing of GRM5 in humans gives rise to two major isoforms that also differ in C-terminal length; the longer of the two, human mGlu 5 a (equivalent to rat $\mathrm{mGlu}_{5} \mathrm{~b}$ ) has a 32-amino acid insertion after residue 876 but is otherwise identical to human $\mathrm{mGlu}_{5} \mathrm{~b}$ (equivalent to rat $\mathrm{mGlu}_{5}$ a) (Minakami et al., 1993, 1995). Variations in C-terminal length due to alternative splicing of group I receptors influences surface expression, subcellular localization, dimerization, interactions with intracellular proteins, and ultimately cellular responses (Joly et al., 1995; Mion et al., 2001; Francesconi and Duvoisin, 2002; Kumpost et al., 2008; Tateyama and Kubo, 2008; Francesconi et al., 2009a; Techlovská et al., 2014). 


\section{B. Localization and Signal Transduction}

The group I mGlu receptors are predominantly found in postsynaptic neurons within the CNS (Fig. 2), increasing neuronal excitability and membrane depolarization when activated. In certain circuits, group I mGlu receptors can be found on presynaptic terminals, acting as autoreceptors to modulate neurotransmitter release (reviewed in Pittaluga, 2016). Furthermore, group I mGlu receptors are also expressed in glial cells (reviewed in Spampinato et al., 2018). The cellular responses resulting from group I mGlu receptor activation are highly complex and context dependent.

Group I mGlu receptors preferentially couple to the $\mathrm{G}_{\mathrm{q} / 11}$ family of $\mathrm{G}$ proteins, which activate phospholipase $\mathrm{C}$ (PLC) $\beta$, which hydrolyses phosphatidylinositol 4,5bisphosphate in the membrane to yield the second messengers: diacylglycerol and inositol 1,4,5-trisphosphate, mobilizing intracellular $\mathrm{Ca}^{2+}\left(\mathrm{iCa}^{2+}\right)$ stores

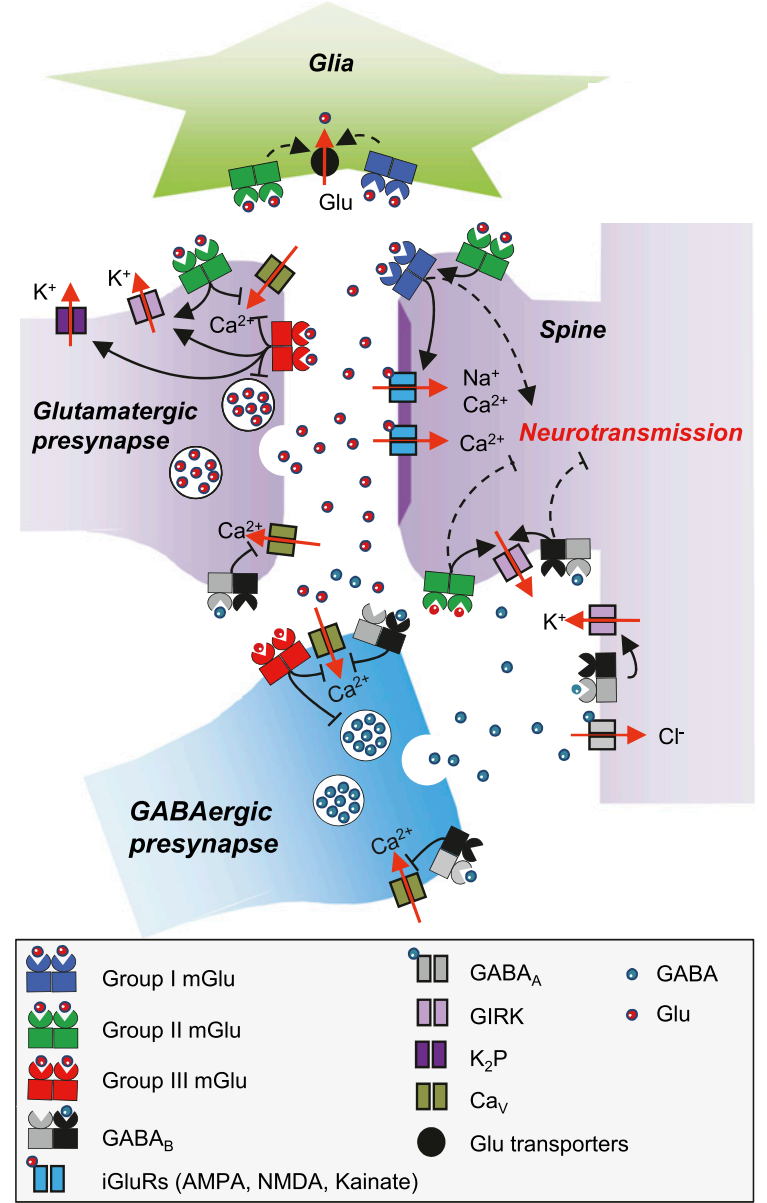

Fig. 2. Synaptic and nonneuronal localization of mGlu receptor subtypes. General overview of metabotropic glutamate receptor neuronal and glial cell localization within glutamatergic and GABAergic synapses. Relationships with other key receptors and transporters that recognize glutamate and GABA as well as ion channels involved in neurotransmission are also shown. Glutamate transporters represent multiple types, namely, excitatory amino acid transporters (EAATs): EAAT1 (also referred to as glutamate aspartate transporter) and EAAT2 (also known as glutamate transporter 1 or solute carrier family 1 member 2 ). The following abbreviations are used: $\mathrm{Ca}_{\mathrm{V}}$, voltage-gated $\mathrm{Ca}^{2+}$ channel; iGluRs, ionotropic glutamate receptors; K2P, two-pore domain $\mathrm{K}^{+}$channel.
(Sugiyama et al., 1987) (Fig. 3). Potentiation of $\mathrm{mGlu}_{5}$ receptors increases phosphoinositide hydrolysis in vivo in different mouse brain areas, such as prefrontal cortex, cerebellum, hypothalamus, hippocampus, and striatum (Zuena et al., 2018). Interestingly, endogenous activation of $\mathrm{mGlu}_{5}$ receptors largely accounts for basal phosphoinositide hydrolysis particularly in the prefrontal cortex. Downstream of these second messengers, activation of protein kinase $\mathrm{C}$ (PKC) and calmodulin triggers signaling cascades that ultimately phosphorylate and activate extracellular signal-regulated kinases 1 and $2($ ERK1/2), which regulate gene transcription associated with synaptic plasticity (long-term depression or long-term potentiation) (Servitja et al., 1999, 2003; Kanumilli et al., 2002; Page et al., 2006; Jin et al., 2013a; Hong et al., 2016). Diacylglycerol can be further broken down by diacylglycerol lipase to yield endogenous cannabinoid, 2-arachidonoylglycerol (Jung et al., 2005; Gregg et al., 2012). Beyond $\mathrm{G} \alpha_{\mathrm{q} / 11}$-mediated signaling, $\beta \gamma$ subunits can enhance or inhibit $\mathrm{Ca}^{2+}$ and $\mathrm{K}^{+}$channels, including ionotropic glutamate receptors, to modulate neuronal excitability and membrane potential (reviewed in Valenti et al., 2002), through physical interactions mediated by scaffolding proteins (Tu et al., 1999) or indirect mechanisms driven by intracellular effectors (Fig. 3). The $\beta \gamma$ subunits can also activate phosphoinositide-3-kinase (PI3K), which in turn activates RAC-alpha serine/threonine-protein kinase (Akt)-dependent signaling cascades implicated in protein synthesis-dependent long-term depression and cell survival (Hou and Klann, 2004; Page et al., 2006; Hullinger et al., 2015; Zhu et al., 2018). In recombinant systems $\mathrm{mGlu}_{1}$ and $\mathrm{mGlu}_{5}$ receptors also couple to $\mathrm{G} \alpha_{\mathrm{s}}$, stimulating adenylyl cyclases (ACs) and increasing cAMP production (Aramori and Nakanishi, 1992; Joly et al., 1995; Francesconi and Duvoisin, 1998, 2000; Nasrallah et al., 2018). In addition, group I receptors signal via $G$ protein-independent mechanisms (e.g., Homer, src kinases, arrestins, transactivation of tyrosine kinases) to activate different kinase cascades that contribute to synaptic plasticity (Iacovelli et al., 2003; Yang et al., 2006; Emery et al., 2010; Kubota et al., 2014; Eng et al., 2016). Downstream of group I receptor activation diverse transcription factors are activated, including cAMP response element-binding protein (Mao and Wang, 2003b), Elk-1 (Mao and Wang, 2003a; Jong et al., 2005, 2009), c-Jun (Jong et al., 2009), and serum response factor (Kumar et al., 2012).

The mechanisms that regulate group I mGlu receptor activity are equally complex (Fig. 3). The C termini of group I receptors contain binding sites for diverse scaffolding proteins that regulate receptor localization and recycling in addition to directly linking group I mGlu receptors to other receptors and channels within the postsynaptic density (Roche et al., 1999; Kitano et al., 2002; Lee et al., 2008; Wang et al., 2009; Hu et al., 2012; Wagner et al., 2015; 


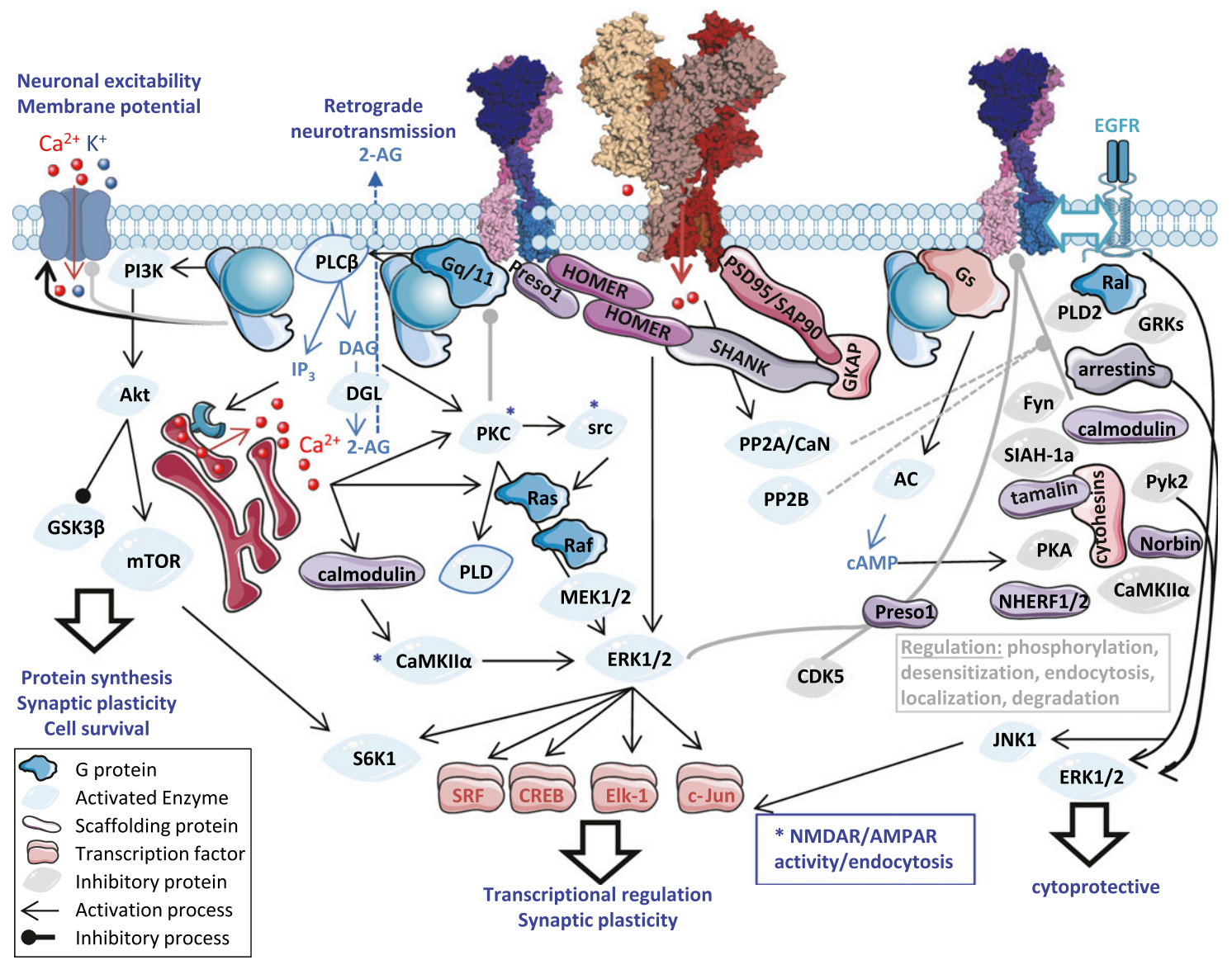

Fig. 3. Signal transduction and regulation of group I mGlu receptors. Overview of group I mGlu receptor scaffolding partners, transducers, downstream effectors, and regulatory proteins; refer to main text for associated primary references. Blue bolded text indicates physiologic consequences linked to specific intracellular responses. The following abbreviations are used: AC, adenylyl cyclase; 2-AG, 2-arachidonoylglycerol; $\mathrm{CaMK}, \mathrm{Ca}^{2+} /$ calmodulin-dependent protein kinase; CREB, cAMP response element-binding protein; DAG, diacylglycerol; DGL, diacylglycerol lipase; EGFR, epidermal growth factor receptor; GKAP, guanylate kinase-associated protein; GRK, G protein-coupled receptor kinase; IP ${ }_{3}$, inositol 1,4,5trisphosphate; JNK, c-Jun N-terminal kinase; MEK1/2, mitogen-activated protein kinase kinases 1 and 2; mTOR, mammalian target of rapamycin; NHERF1/2, $\mathrm{Na}^{+} / \mathrm{H}^{+}$exchange regulatory cofactors 1 and 2; PKA, protein kinase A; PLD, phospholipase D; PSD-95, postsynaptic density protein 95 (also known as SAP-90 for synapse-associated protein 90); PP2B, protein phosphatase 2B; PP2A/CaN, protein phosphatase 2A/calcineurin; S6K1, ribosomal protein S6 kinase $\beta$-1; SHANK, SH3 and multiple ankyrin repeat domains protein; SIAH-1a, E3 ubiquitin-protein ligase SIAH1A; SRF, serum response factor.

Eng et al., 2016; Gulia et al., 2017). Second messengeractivated kinases also provide negative feedback regulating cellular responses ( $\mathrm{iCa}^{2+}$ oscillations or receptor desensitization) through phosphorylation of intracellular loops and/or the C terminus (Kawabata et al., 1996; Gereau and Heinemann, 1998; Bhattacharya et al., 2004; Mundell et al., 2004; Kim et al., 2005; Bradley and Challiss, 2011; Ko et al., 2012; Jin et al., 2013a,b, 2018; Raka et al., 2015; Uematsu et al., 2015; Vergouts et al., 2017; Yang et al., 2017; Marks et al., 2018). However, not all functional responses are equally influenced. For example, PKC phosphorylation of $\mathrm{mGlu}_{1}$ receptors desensitizes signaling to accumulation of inositol phosphate but not cAMP (Francesconi and Duvoisin, 2000). Select kinases bind the $\mathrm{C}$ terminus and/or phosphorylate the receptor altering effector coupling, ability to bind other proteins, or receptor endocytosis (Dale et al., 2000; Sallese et al., 2000; Iacovelli et al., 2003; Pula et al., 2004; Nicodemo et al., 2010; Jin et al., 2017). By example, Preso1 enhances cyclin-dependent kinase 5 and ERK1/2 phosphorylation of the Homer binding site within the $\mathrm{C}$ terminus ( $\mathrm{Hu}$ et al., 2012), whereas calmodulin and E3 ubiquitin-protein ligase SIAH-1a recognize overlapping sites in the $\mathrm{C}$ tail (Ishikawa et al., 1999), with PKC phosphorylation of this site enhancing SIAH-1a but inhibiting calmodulin binding (Ko et al., 2012). Second messenger-dependent kinases are critical for group I mGlu receptor-dependent long-term depression and potentiation by modulating the activity or promoting endocytosis of ionotropic glutamate receptors including $N$-methyl-D-aspartate (NMDA) and $\alpha$-amino-3-hydroxy-5-methyl-4-isoxazolepropionic acid (AMPA) receptor subtypes (Jia et al., 1998; Snyder et al., 2001; Benquet et al., 2002; Moult et al., 2006; Jin et al., 2013b, 2015; Xu et al., 2013), although PKC-independent mechanisms for NMDA receptor potentiation by group I mGlu receptors have also been reported (Harvey and Collingridge, 1993; Kinney and Slater, 1993; Rahman and Neuman, 1996). In a reciprocal fashion, NMDA receptor-mediated 
stimulation of protein phosphatase $2 \mathrm{~A} /$ calcineurin acts to regulate recycling of group I mGlu receptors (Alagarsamy et al., 2005; Pandey et al., 2014), with protein phosphatase $2 \mathrm{~B}$ playing a similar role (Mahato et al., 2015). It is clear that activation of group I mGlu receptors can trigger a complex network of intracellular effectors that encode the cellular responses that give rise to complex physiologic effects from synaptic plasticity to cell survival.

When coexpressed within the same cell population, activation of $\mathrm{mGlu}_{1}$ versus $\mathrm{mGlu}_{5}$ receptors can lead to distinct cellular outcomes (Valenti et al., 2002). Moreover, postsynaptic group I mGlu receptor activation can result in modulation of neurotransmitter release from presynaptic cells via retrograde signaling, for example, endocannabinoids or nitric oxide (Fig. 3) (Maejima et al., 2001; Robbe et al., 2002; Sergeeva et al., 2007; Gregg et al., 2012; Aubrey et al., 2017; Xiang et al., 2019). As a further layer of complexity, both group I mGlu receptors form heteromers and/or larger-order oligomers with other G protein-coupled receptors (GPCRs) (discussed in further detail later), which alters intracellular signaling profiles.

\section{Pathophysiology and Therapeutic Potential}

A number of reviews provide in depth coverage of the distribution, physiology, and pathophysiological roles of group I mGlu receptors (Ferraguti and Shigemoto, 2006; Niswender and Conn, 2010; Golubeva et al., 2016; Crupi et al., 2019; Pereira and Goudet, 2019). Inhibitors and activators of the individual subtypes are being pursued for a myriad of different psychiatric and neurologic disorders. Briefly, and of relevance to the pharmacological agents reviewed in depth below, distribution of $\mathrm{mGlu}_{1}$ receptors in regions associated with pain

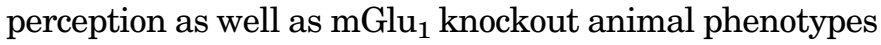
suggests $\mathrm{mGlu}_{1}$ inhibitors are potential therapeutic agents for neuropathic pain (Neugebauer, 2002; Schkeryantz et al., 2007). Preclinical studies also indicate $\mathrm{mGlu}_{1}$ receptor inhibitors may have therapeutic benefit in treating seizures, addiction, anxiety, and certain cancers (Namkoong et al., 2006; Dravolina et al., 2017). $\mathrm{mGlu}_{5}^{-1-}$ mice have deficits in prepulse inhibition (Brody et al., 2004), impaired learning and memory (Xu et al., 2009; Zeleznikow-Johnston et al., 2018), and reduced propensity for addiction/abuse (Chiamulera et al., 2001) and reverse the majority of fragile $\mathrm{X}$ syndrome phenotypes in preclinical models (Dölen and Bear, 2008). As such, selective $\mathrm{mGlu}_{5}$ receptor inhibitors are desired in the setting of depression (Chaki and Fukumoto, 2018) and anxiety (Ferraguti, 2018) as well as neurodevelopmental disorders such as fragile $\mathrm{X}$ syndrome (Yamasue et al., 2019). In this respect multiple $\mathrm{mGlu}_{5}$ receptor inhibitors have entered phase 2 clinical trials as discussed in detail later; however, to date none have reached market. Potentiation or activation of both group I receptors offers the promise for treating the positive and cognitive symptoms associated with schizophrenia (Walker and Conn, 2015; Nicoletti et al., 2019).

Beyond neurologic and psychiatric disorders, inhibition of $\mathrm{mGlu}_{1}$ receptors is neuroprotective (in vitro and in vivo) after oxygen-glucose deprivation or ischemic insult (Henrich-Noack et al., 1998; Pellegrini-Giampietro et al., 1999) and may therefore offer a novel intervention for stroke. For multiple preclinical models of neurodegenerative diseases, genetic ablation or pharmacological inhibition of $\mathrm{mGlu}_{5}$ receptors is neuroprotective and treats associated symptoms, for example, acting procognitively in Alzheimer's disease or Huntington's disease (reviewed in Ribeiro et al., 2017) or improving motor deficits in amyotrophic lateral sclerosis (reviewed in Battaglia and Bruno, 2018) or Parkinson's disease (Mazur, 1995; Battaglia et al., 2004; Armentero et al., 2006; Ambrosi et al., 2010; Black et al., 2010; Masilamoni et al., 2011; Fuzzati-Armentero et al., 2015), although mGlu ${ }_{5}$ receptor activators/potentiators may also treat cognitive symptoms associated with Huntington's disease (Doria et al., 2013, 2015, 2018). Inhibition of $\mathrm{mGlu}_{5}$ receptors is also indicated for treating neurodegeneration associated with drugs of abuse (Battaglia et al., 2002).

\section{Group II: Metabotropic Glutamate Receptors 2 and 3}

\section{A. Receptor Subtypes and Splice Variants}

The group II members, $\mathrm{mGlu}_{2}$ and $\mathrm{mGlu}_{3}$ receptors, were first cloned in rat in 1992 (Tanabe et al., 1992) and a few years later in human (Flor et al., 1995a; Emile et al., 1996). Encoded by the GRM2 gene [ENSG00000164082] and localized in human chromosome 3 and rat chromosome 8 , no splice variants have been described for $\mathrm{mGlu}_{2}$ receptor subtype (Sartorius et al., 2006). In human, the GRM3 gene [ENSG00000198822] encodes the $\mathrm{mGlu}_{3}$ receptor, for which three splice variants are known (Sartorius et al., 2006). The most abundant GRM3 variant lacks exon 4 (GRM3Delta4), encoding a truncated membraneassociated protein that retains the extracellular VFT but lacks the 7TM, which is replaced with a unique 96-amino acid $\mathrm{C}$ terminus. mGlu $\mathrm{Bdelta}_{4}$ can bind orthosteric ligands and interact with the full-length protein and may thus have a dominant negative effect (GarcíaBea et al., 2017). Spontaneous mutations in $\mathrm{mGlu}_{3}$ are associated with melanoma (Prickett et al., 2011; Neto and Ceol, 2018), whereas single nucleotide polymorphisms in GRM3 are linked to cognitive performance in individuals with schizophrenia and are postulated to influence pharmacotherapy (reviewed in Maj et al., 2016 and Saini et al., 2017).

\section{B. Localization and Signal Transduction}

The group II mGlu receptors are located both pre- and postsynaptically, with $\mathrm{mGlu}_{3}$ receptors also found in 
glial cells throughout the brain (Fotuhi et al., 1994; Testa et al., 1994) (Fig. 2). Using a radiolabeled orthosteric agonist selective for group II receptors, $(1 R, 2 S, 4 R, 5 R, 6 R)$-2-amino-4-fluorobicyclo[3.1.0] hexane-2,6-dicarboxylic acid, in conjunction with knockout mice for either subtype, $\mathrm{mGlu}_{3}$ receptor levels were found to be generally higher than $\mathrm{mGlu}_{2}$ receptors in forebrain regions but similar within the striatum (Wright et al., 2013). In the thalamus and hippocampus, the two subtypes exhibit distinct and, for some nuclei/circuits, segregated expression patterns (Wright et al., 2013). Dissecting the relative contribution of $\mathrm{mGlu}_{2}$ versus $\mathrm{mGlu}_{3}$ receptor subtypes has presented a major challenge due to the lack of subtype-selective pharmacological tools (discussed in further detail below).

Group II mGlu receptors preferentially couple to $\mathrm{G}_{\mathrm{i} / \mathrm{o}}$ proteins, inhibiting adenylyl cyclase and cAMP production as well as inhibiting guanylate cyclase and cGMP production (Wroblewska et al., 2006) (Fig. 4). On presynaptic terminals, $\beta \gamma$ subunits modulate ion channel function, inhibiting $\mathrm{N}$-type $\mathrm{Ca}^{2+}$ channels (McCool et al., 1996) and activating G protein-coupled inwardly rectifying potassium channel (GIRK) channels (Knoflach and Kemp, 1998; Sharon et al., 1997), thereby decreasing exocytosis of vesicles containing glutamate (Macek et al., 1998; Flavin et al., 2000; Olivero et al., 2017), GABA (Hayashi et al., 1993; Gereau and Conn, 1995; Salt and Eaton, 1995; Schaffhauser et al., 1998) and dopamine (Feenstra et al., 1998; Verma and Moghaddam, 1998; Johnson et al., 2017). The $\beta \gamma$ subunits can also activate PI3Kdependent activation of Akt with downstream effectors regulating protein synthesis and gene transcription involved in cytoprotection and synaptic plasticity (Aronica et al., 2003; Ciccarelli et al., 2007; Durand et al., 2011; Li et al., 2015; Ritter-Makinson et al., 2017; Xing et al., 2018). Furthermore, group II receptor activation can trigger transactivation of insulin growth factor-1 receptors via $\beta \gamma$ subunit activation of PLC and focal adhesion kinase, leading to ERK1/2 phosphorylation (Hu et al., 2019), a key integrator of multiple convergent pathways that shapes the overall cellular response (Aronica et al., 2003; Ciccarelli et al., 2007). In postsynaptic membranes, group II receptor activation regulates trafficking of NMDA or AMPA receptors with different mechanisms implicated: PKC, soluble $\mathrm{N}$-éthylmaleimide-sensitive-factor attachment protein receptor complexes, Akt/glycogen synthase kinase (GSK) $3 \beta$ (Tyszkiewicz et al., 2004; Xi et al., 2011; Cheng et al., 2013). Furthermore, $\mathrm{mGlu}_{3}$ receptordependent long-term depression (LTD) in the prefrontal cortex is mediated by functional cross-talk between $\mathrm{mGlu}_{3}$ and $\mathrm{mGlu}_{5}$ receptors on postsynaptic neurons (Fig. 2). Activation of $\mathrm{mGlu}_{3}$ receptors releases $\beta \gamma$ subunits to enhance mGlu $_{5}$ receptor coupling to $\mathrm{G}_{\mathrm{q}}(\mathrm{Di}$ Menna et al., 2018). Cross-talk between $\mathrm{mGlu}_{3}$ and
$\mathrm{mGlu}_{5}$ receptors is postulated to account for the reported dependence on PLC and PKC for $\mathrm{mGlu}_{3}$ receptor-dependent LTD (Otani et al., 2002; Huang et al., 2007). Recently, however, $\mathrm{mGlu}_{3}$ receptordependent LTD was shown to be modulated by mGlu receptor interactions with homer and signaling via PI3K, Akt, and GSK3 $\beta$ to result in AMPA receptor internalization, a mechanism disrupted by acute stress (Joffe et al., 2019). Indeed, the cellular context is an important contributor to shaping the cellular responses instigated by group II receptor activation. For example, in astrocytes, $\mathrm{mGlu}_{3}$ receptor activation results in both decreases and increases in cAMP, whereas in neurons the effect on cAMP levels is purely inhibitory (Moldrich et al., 2002). Astrocytic $\mathrm{mGlu}_{3}$-mediated increases in cAMP levels are dependent on $\mathrm{iCa}^{2+}$ levels (mobilization of stores and extracellular influx) and local release of adenosine, which acts at colocated $\mathrm{G}_{\mathrm{s}}$-coupled adenosine $A_{2 A}$ receptors (Moldrich et al., 2002).

With respect to regulation of group II mGlu receptor activity, the cellular context is also a major contributor. Phosphorylation of intracellular loops and/or the C-terminal tail by protein kinase A, PKC, and G protein-coupled receptor kinases have a central role in receptor regulation (Kamiya and Yamamoto, 1997; Macek et al., 1998; Schaffhauser et al., 2000; Cai et al., 2001), uncoupling receptors from $G$ proteins, and promoting interactions with scaffolding proteins such as arrestins, which mediate receptor endocytosis (Iacovelli et al., 2009). The C-terminal tail of group II mGlu receptors binds to multiple scaffolding proteins including protein interacting with $\mathrm{C}$ kinase, glutamate receptor-interacting protein, tamalin, $\mathrm{Na}^{+} / \mathrm{H}^{+}$exchange regulatory cofactors 1 and 2 , and Ran-binding protein microtubule-organizing center (Hirbec et al., 2002; Kitano et al., 2002; Seebahn et al., 2008; RitterMakinson et al., 2017), as well as protein phosphatase $2 \mathrm{C}$, which dephosphorylates $\mathrm{mGlu}_{3}$ receptors (Flajolet et al., 2003). Interactions between group II mGlu receptors with diverse intracellular scaffolding proteins regulates receptor localization and functional responses, which likely governs differences observed between cell types or for neurons from different brain regions. Interestingly, $\mathrm{mGlu}_{2}$, but not $\mathrm{mGlu}_{3}$, receptors are reportedly resistant to homologous desensitization by $\mathrm{G}$ protein-coupled receptor kinases with respect to cAMP signaling (Iacovelli et al., 2009), although heterologous mechanisms, for example, due to PKC activation by colocated adenosine $\mathrm{A}_{3}$ receptors, affect both subtypes (Macek et al., 1998; Lennon et al., 2010). Functional cross-talk between $\mathrm{mGlu}_{2}$ receptors and colocated serotonin receptors $2 \mathrm{~A}\left(5-\mathrm{HT}_{2 \mathrm{~A}}\right)$ can also modulate cellular responses to activation of either receptor (Marek et al., 2000; Molinaro et al., 2009; Murat et al., 2019). The interplay of intracellular effectors stimulated by group II mGlu receptors, coupled with regulatory proteins as well as coexpression of 


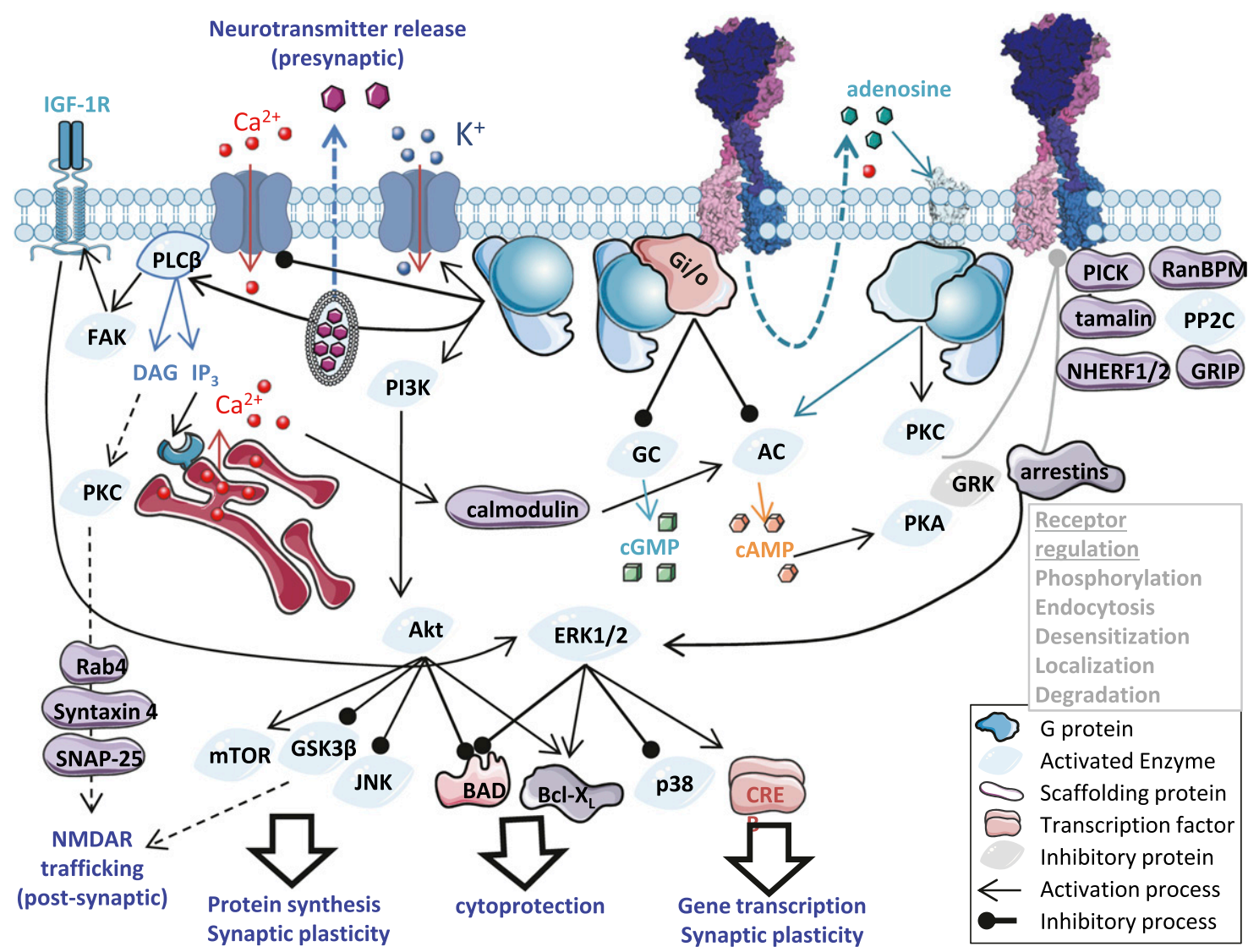

Fig. 4. Signal transduction and regulation of group II mGlu receptors. Overview of group II mGlu receptor scaffolding partners, transducers, downstream effectors, and regulatory proteins; refer to main text for associated primary references. Blue bolded text indicates physiologic consequences linked to specific intracellular responses. The following abbreviations are used: AC, adenylyl cyclase; CREB, cAMP response elementbinding protein; DAG, diacylglycerol; FAK, focal adhesion kinase; GC, guanylate cyclase; GRIP, glutamate-receptor interacting protein; GRK, G protein-coupled receptor kinase; IGF-1R, insulin growth factor-1 receptor; $\mathrm{IP}_{3}$, inositol 1,4,5-trisphosphate; JNK, c-Jun N-terminal kinase; mTOR, mammalian target of rapamycin; NHERF $1 / 2, \mathrm{Na}^{+} / \mathrm{H}^{+}$exchange regulatory cofactors 1 and 2 ; NMDAR, NMDA receptor; PICK1, protein interacting with C kinase; PKA, protein kinase A; PP2C, protein phosphatase 2C; RanBPM, Ran-binding protein microtubule-organizing center; SNAP-25, synaptosomal-associated protein $25 \mathrm{kDa}$.

other cell surface receptors, gives rise to cell typespecific roles for group II mGlu receptors.

\section{Pathophysiology and Therapeutic Potential}

The expression patterns of group II mGlu receptors (reviewed in Ferraguti and Shigemoto, 2006) coupled with phenotypes of knockout animals suggest that group II receptors are attractive therapeutic targets for psychosis, cognition, anxiety, pain, and addiction (Cross et al., 2018; Mazzitelli et al., 2018; Pereira and Goudet, 2019), although for many indications establishing whether $\mathrm{mGlu}_{2}$ or $\mathrm{mGlu}_{3}$ receptors are the best target in preclinical models of disease has been challenging due to a lack of subtype-selective agents. Despite this shortcoming, both agonists and potentiators of group II receptors have been actively pursued, with multiple agents entering phase 2 or 3 for treating schizophrenia and addiction disorders (Nicoletti et al., 2019). Conversely, group II receptors inhibitors are promising interventions for depression and anxiety and as neuroprotective agents in the setting of ischemia (Celanire et al., 2015; Motolese et al., 2015). However, activation of group II receptors is neuroprotective after excitotoxic insults (Battaglia et al., 2003). In particular, activation of astrocytic $\mathrm{mGlu}_{3}$ receptors confers neuroprotection to various insults (in vitro and in vivo) via paracrine mechanisms (Bruno et al., 1997; Corti et al., 2007; Cippitelli et al., 2010; Caraci et al., 2011), offering a potential therapeutic target for neurodegenerative diseases and psychiatric conditions associated with neuronal death.

\section{Group III: Metabotropic Glutamate Receptors $4,6,7$, and 8}

\section{A. Receptor Subtypes and Splice Variants}

Before molecular cloning, group III mGlu receptors were termed L-(+)-2-amino-4-phosphonobutyric acid (L-AP4) receptors due to high sensitivity to this ligand, which depresses synaptic transmission in the brain and in retina (Thomsen, 1997). In the 1990s, molecular cloning revealed that four different receptor subtypes mediated the biologic effects of L-AP4: $\mathrm{mGlu}_{4}, \mathrm{mGlu}_{6}$, $\mathrm{mGlu}_{7}$, and $\mathrm{mGlu}_{8}$ receptors. The $\mathrm{mGlu}_{4}$ receptor is 
encoded by the GRM4 gene [ENSG00000124493], which is localized in human chromosome 6 , rat chromosome 20, and mouse chromosome 17 (Tanabe et al., 1992; Flor et al., 1995b). Two main splice variants were predicted, termed $\mathrm{mGlu}_{4 \mathrm{a}}$ and ${ }_{4 \mathrm{~b}}$ (Thomsen et al., 1997), but further studies failed to detect $\mathrm{mGlu}_{4 \mathrm{~b}}$ in different rat brain areas, and no splice site consensus sequences that could support its existence were found in human genomic sequence containing the whole GRM4 gene, suggesting $\mathrm{mGlu}_{4 \mathrm{~b}}$ corresponds to a recombination artifact (Corti et al., 2002). Another variant lacking the first 128 base pairs, termed taste $\mathrm{mGlu}_{4}$, is found in rat taste buds. The corresponding protein is predicted to lack approximately half the extracellular domain, including a large portion of the glutamate-binding domain (Chaudhari et al., 2000). First cloned in 1993, the mGlu 6 receptor is encoded by GRM6 gene [ENSG00000113262] localized in human chromosome 5 (Nakajima et al., 1993). Two splice variants, termed $\mathrm{mGlu}_{6 \mathrm{~b}}$ and $\mathrm{mGlu}_{6 \mathrm{c}}$, are found in both human and rats, which correspond to truncated $\mathrm{mGlu}_{6}$ receptors lacking the transmembrane domain and intracellular portions of the receptor (Valerio et al., 2001a,b). The $\mathrm{mGlu}_{7}$ receptor is encoded by the GRM7 gene, localized in rat chromosome 4 and in human chromosome 3 (Okamoto et al., 1994; Saugstad et al., 1994; Makoff et al., 1996). Two main variants, $\mathrm{mGlu}_{7} \mathrm{a}$ and $\mathrm{mGlu}_{7} \mathrm{~b}$, in rats and humans have different C-terminal tails. The last 16 residues of mGlu $_{7 a}$ are substituted by 23 different residues in mGlu $_{7 b}$ due to the insertion of an out-of-frame 92-base pair exon (Flor et al., 1997; Corti et al., 1998). Three other isoforms have been described, named v3, v4, and v5 (Schulz et al., 2002). Although $\mathrm{mGlu}_{7 \mathrm{a}}$ and $\mathrm{mGlu}_{7 \mathrm{~b}}$ receptor variants are primarily expressed in the CNS, the expression of v3 and v4 isoforms appears to be restricted in nonneuronal tissues (Schulz et al., 2002). The mGlu receptor, encoded by the GRM8 gene [ENSG00000179603] found in human chromosome 7, was originally cloned in mouse in 1995 (Duvoisin et al., 1995) followed by human (Scherer et al., 1997; Wu et al., 1998) and rat (Saugstad et al., 1997). Three splice variants have been described: $\mathrm{mGlu}_{8 \mathrm{a}}$, mGlu $8 \mathrm{~b}$, and $\mathrm{mGlu}_{8 \mathrm{c}}$ (Corti et al., 1998; Malherbe et al., 1999). The last 16 residues of the C-terminal tails of the $\mathrm{mGlu}_{8 \mathrm{a}}$ and $\mathrm{mGlu}_{8 \mathrm{~b}}$ receptors are different, whereas the $\mathrm{mGlu}_{8 \mathrm{c}}$ variant is a truncated receptor lacking the transmembrane domains and intracellular C tail. For each of the group III mGlu receptors the different splice variants show distinct tissue distribution and/or changes in intracellular portions of the receptor, which have the potential to alter signal transduction pathways triggered in response to receptor activation.

\section{B. Localization and Signal Transduction}

Most group III mGlu receptors are widely expressed throughout the CNS, with the exception of $\mathrm{mGlu}_{6}$, which is mostly restricted to the retina (reviewed in
Ferraguti and Shigemoto, 2006). Group III mGlu receptors are also expressed in glial cell types in the brain, with the exception of $\mathrm{mGlu}_{7}$ (reviewed in Spampinato et al., 2018). Outside the CNS, group III mGlu receptor expression has been reported in kidney, pancreas, liver, cells from the immune system, and bones, for example (see Julio-Pieper et al., 2011, for review).

In the $\mathrm{CNS}, \mathrm{mGlu}_{4}, \mathrm{mGlu}_{7}$, and $\mathrm{mGlu}_{8}$ receptors are mainly expressed in the active zone of presynaptic glutamatergic and GABAergic neurons (Kinoshita et al., 1996a; Shigemoto et al., 1997; Wada et al., 1998; Corti et al., 2002; Ferraguti et al., 2005; Ferraguti and Shigemoto, 2006) (Fig. 2). Group III receptors can act as autoreceptors reducing glutamate release in the synaptic cleft and as heteroreceptors reducing the release of GABA (Schoepp, 2001). $\mathrm{mGlu}_{4}, \mathrm{mGlu}_{7}$, and $\mathrm{mGlu}_{8}$ receptors are preferentially coupled to heterotrimeric $\mathrm{G}_{\mathrm{i} / \mathrm{o}}$ proteins, leading to the inhibition adenylyl cyclase production of cAMP via the $\mathrm{G} \alpha$ subunit (Fig. 5). On presynaptic terminals, $\mathrm{mGlu}_{4}, \mathrm{mGlu}_{7}$, or $\mathrm{mGlu}_{8}$ receptors are well documented to inhibit neurotransmitter release through a direct $\beta \gamma$ subunit-mediated inhibition of $\mathrm{N}$ or $\mathrm{P} / \mathrm{Q}$ type of voltage-gated $\mathrm{Ca}^{2+}$ channels (Anwyl, 1999; Perroy et al., 2000; Millán et al., 2002a,b; Capogna, 2004; Martín et al., 2007). In addition, $\mathrm{mGlu}_{4}$ and $\mathrm{mGlu}_{7}$ receptors also inhibit voltage-gated $\mathrm{Ca}^{2+}$ channels via a PKC-dependent mechanism (Perroy et al., 2000; Abitbol et al., 2012), involving interactions between calmodulin and the scaffolding protein, protein interacting with $\mathrm{C}$ kinase, for $\mathrm{mGlu}_{7}$ receptors (Perroy et al., 2002; Suh et al., 2013). Activation of $\mathrm{mGlu}_{4}, \mathrm{mGlu}_{7}$, or $\mathrm{mGlu}_{8}$ receptors can also decrease neuronal excitability by the released $\beta \gamma$ subunits acting on GIRK channels (Dutar et al., 1999; Saugstad et al., 1996). Other studies suggest that group III mGlu receptors may activate background $\mathrm{K}^{+}$ channels such as TREK1 and TREK2 (potassium channel subfamily $\mathrm{K}$ member 2 and 10), thereby further decreasing neuronal activity (Lesage et al., 2000; Cain et al., 2008). Group III mGlu receptors are also proposed to inhibit neurotransmitter vesicle exocytosis through direct interactions with the release machinery (Chavis et al., 1998; Erdmann et al., 2012). Coupling of group III receptors to PI3K, probably through $\mathrm{G} \beta \gamma$ subunits, and mitogen-activated protein kinase is implicated in neuroprotection mechanisms (Iacovelli et al., 2002).

Among group III mGlu receptors, the $\mathrm{mGlu}_{6}$ receptor is distinct as its expression is mostly restricted to postsynaptic bipolar ON neurons in the retina (Nomura et al., 1994; Vardi et al., 2000), with no strong expression detected in the brain (Nakajima et al., 1993). The mGlu receptor is critical for glutamate-induced signaling in ON-bipolar cells in the retina during darkness (Nomura et al., 1994; Vardi et al., 2000). Glutamate, released from rod photoreceptors in the dark, activates postsynaptic $\mathrm{mGlu}_{6}$ receptors in bipolar ON cells that lead to the closure of a nonselective ion channel, TRPM1-L 
[a long form transcript of transient receptor potential cation channel subfamily $M$ member 1 (TRPM1) expressed solely in the dendritic tip of bipolar ON neurons] (Koike et al., 2010). TRPM1 inhibition results in hyperpolarization of bipolar ON neurons, thus inhibiting the ON pathway into darkness. The signaling cascade involves Go $\alpha$ (Koike et al., 2010), $\mathrm{G} \beta \gamma$ (Shen et al., 2012), and other proteins such as the orphan GPCR GPR179 or the interacting protein Nyctalopin (Zeitz et al., 2015) (Fig. 5). Collectively, the intracellular signal transduction and regulatory pathways engaged by group III receptor subtypes have not been as well elucidated as group I and group II counterparts.

\section{Pathophysiology and Therapeutic Potential}

The phenotypes of mice lacking the group III mGlu receptors have revealed physiologic roles and potential as therapeutic targets in several neurologic disorders. Mice lacking the $\mathrm{mGlu}_{4}$ receptor present deficits in motor performance, spatial memory, and learning of complex motor tasks (Pekhletski et al., 1996; Gerlai et al., 1998), in accordance with its particularly high expression in the cerebellum (Kinoshita et al., 1996b; Corti et al., 2002). The $\mathrm{mGlu}_{4}^{-1-}$ mice also have enhanced seizure-associated vulnerability (Pitsch et al., 2007) and lack the motor stimulant effect of ethanol (Blednov et al., 2004). The sensitivity to strong noxious stimuli of $\mathrm{mGlu}_{4}^{-1-}$ mice is altered, and nociceptive behavior in the inflammatory phase of the formalin test is accelerated (Vilar et al., 2013). Knockout phenotypes together with preclinical studies highlight $\mathrm{mGlu}_{4}$ receptor as a potential therapeutic target in anxiety and depression (Kalinichev et al., 2014), schizophrenia (Wierońska et al., 2012a), epilepsy (Pitsch et al., 2007; Ngomba et al., 2008), neuroinflammation (Fallarino et al., 2010), autism spectrum disorder (Becker et al., 2014), and chronic pain (reviewed in Pereira and Goudet, 2019). In particular, targeting $\mathrm{mGlu}_{4}$ receptor for the treatment of Parkinson's disease (PD) has attracted much attention (Célanire and Campo, 2012; Amalric et al., 2013; Charvin, 2018; Volpi et al., 2018). Preclinical studies showed that $\mathrm{mGlu}_{4}$ receptor activation corrects the imbalance of neurotransmission among the basal ganglia circuitry that is associated with PD (Charvin et al., 2018b), as shown primarily with $\mathrm{mGlu}_{4}$ potentiation (Marino et al., 2003b) or later with selective agonists (Marino et al., 2003b; Beurrier et al., 2009). Activation or potentiation of $\mathrm{mGlu}_{4}$ receptors also has neuroprotective effects (Copani et al., 1995; Battaglia et al., 2006). Unfortunately, despite promising preclinical results (Charvin et al., 2017, 2018a), the $\mathrm{mGlu}_{4}$ receptor potentiator, foliglurax, recently failed to show sufficient efficacy in a phase II clinical trial for PD.

Comparing the phenotypes of $\mathrm{mGlu}_{4}, \mathrm{mGlu}_{7}$, and $\mathrm{mGlu}_{8}$ knockout mice indicates the $\mathrm{mGlu}_{4}$ receptor is most clearly involved in startle and motivational processes, whereas $\mathrm{mGlu}_{7}$ receptor is involved in hippocampusdependent spatial learning and fear-related behaviors, and $\mathrm{mGlu}_{8}$ receptor deletion yields more subtle behavioral changes and influences body weight (Goddyn et al., 2015). The role of $\mathrm{mGlu}_{7}$ receptors in learning and memory is confirmed by behavioral pharmacology studies (Hikichi et al., 2010a; Klakotskaia et al., 2013). Also, $\mathrm{mGlu}_{7}^{-1-}$ mice and mice lacking functional $\mathrm{mGlu}_{7}$ receptors present an increased susceptibility to seizures
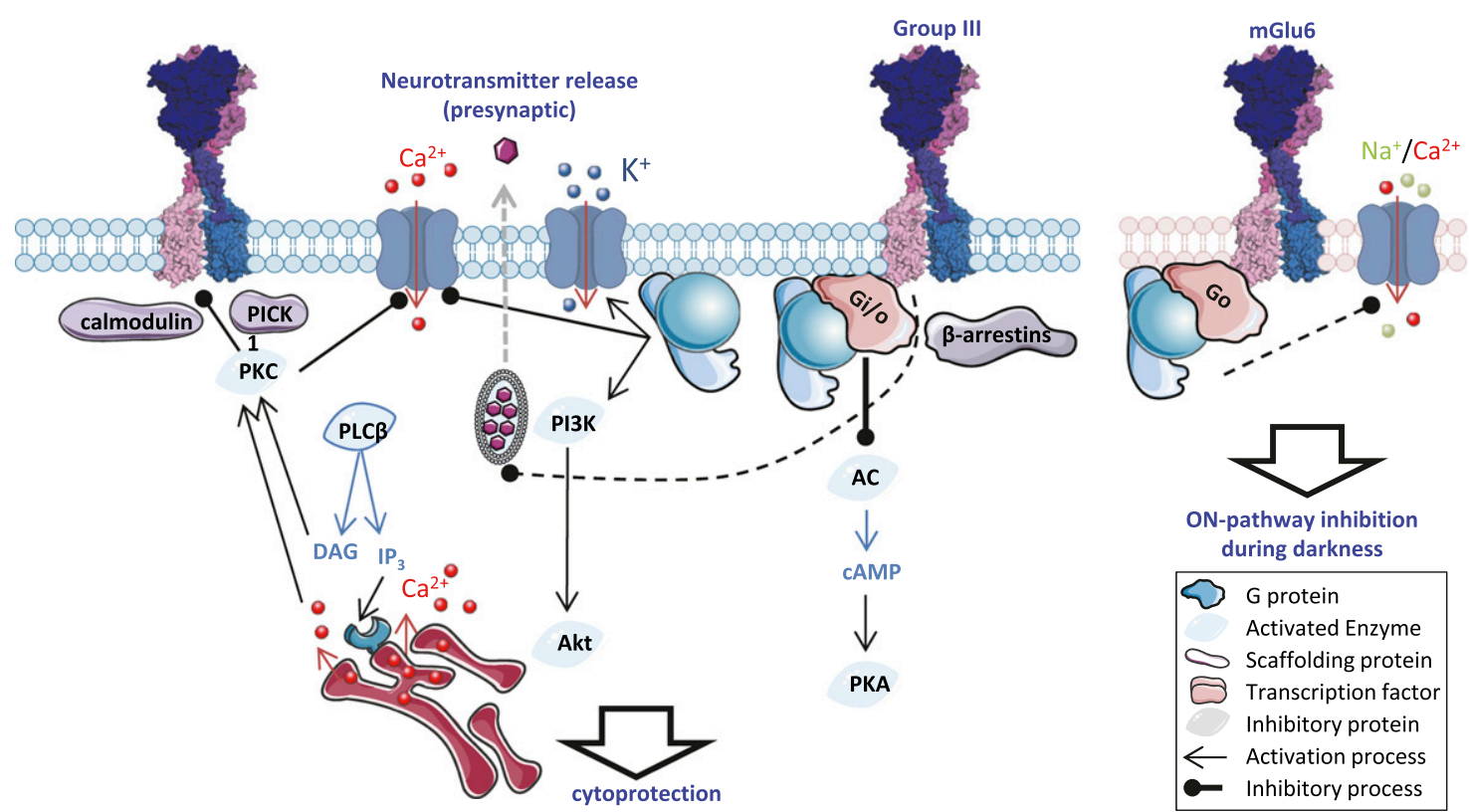

Fig. 5. Signal transduction and regulation of group III mGlu receptors. Overview of group III mGlu receptor scaffolding partners, transducers, downstream effectors, and regulatory proteins; refer to main text for associated primary references. Blue bolded text indicates physiologic consequences linked to specific intracellular responses. The following abbreviations are used: AC, adenylyl cyclase; DAG, diacylglycerol; IP $\mathrm{P}_{3}$, inositol 1,4,5-trisphosphate; PICK1, protein interacting with C kinase; PKA, protein kinase A. 
(Sansig et al., 2001; Bertaso et al., 2008). Accordingly, absence seizures can be induced by the pharmacological blockade of the $\mathrm{mGlu}_{7}$ receptor (Tassin et al., 2016), whereas $\mathrm{mGlu}_{7}$ receptor activation protects against epileptogenesis and epileptic seizures (Girard et al., 2019). Widely expressed in the CNS (Kinzie et al., 1997; Shigemoto et al., 1997; Corti et al., 1998; Kinoshita et al., 1998), the $\mathrm{mGlu}_{7}$ receptor is also considered as a potential therapeutic target for anxiety and depression (Cryan et al., 2003) and neurodevelopmental disorders (O'Connor et al., 2010; Palazzo et al., 2016; Fisher et al., 2018). Genetic disruptions in GRM7 are evident in patients with autism spectrum disorders (Liu et al., 2012; Sanders et al., 2012). Furthermore, the potentiation of $\mathrm{mGlu}_{7}$ receptor activity improves cognitive and social deficits, as well as respiratory impairments, in a mouse model of Rett syndrome (Gogliotti et al., 2017).

Interestingly, depending on the brain structure, $\mathrm{mGlu}_{7}$ and $\mathrm{mGlu}_{8}$ receptors play opposing roles in pain (Boccella et al., 2020). For example, $\mathrm{mGlu}_{7}$ receptor activation in periaqueductal gray and amygdala is pronociceptive, whereas $\mathrm{mGlu}_{8}$ receptor activation is antinociceptive (Marabese et al., 2007; Palazzo et al., 2008). Indeed, for $\mathrm{mGlu}_{7}$ receptors, proalgic or analgesic activity depends on the brain structure and circuits in which the receptor is expressed (see Pereira and Goudet, 2018, for review). For example, activation of $\mathrm{mGlu}_{7}$ receptors in the nucleus accumbens has an antinociceptive effect (Kahl and Fendt, 2016), opposite to the pronociceptive effect when periaqueductal gray $\mathrm{mGlu}_{7}$ receptors are activated (Palazzo et al., 2016). Interestingly, $\mathrm{mGlu}_{7}$ receptor activation also prevents the development of morphine tolerance (Gawel et al., 2018). Additional studies confirmed $\mathrm{mGlu}_{8}$ receptor modulates sensory symptoms associated to neuropathic pain (Rossi et al., 2014). Furthermore, $\mathrm{mGlu}_{8}$ receptor activation in the nucleus tractus solitarius enhances cardiac nociception (Liu et al., 2012). Distinguishing the different physiologic and pathophysiological roles for $\mathrm{mGlu}_{7}$ and $\mathrm{mGlu}_{8}$ receptors has been hampered by a lack of selective pharmacological tools; however, discovery of new pharmacological tools (discussed in further detail later) have aided dissection of different roles and therapeutic indications.

The $\mathrm{mGlu}_{8}$ receptor is one of the least studied mGlu receptor family members, due notably to the lack of selective pharmacological tools. Investigations on the phenotype of genetically modified mice lacking the $\mathrm{mGlu}_{8}$ receptor are thus particularly informative. In various studies, $\mathrm{mGlu}_{8}^{-1-}$ mice exhibit anxiety-related phenotypes. However, some studies report an anxiogeniclike phenotype (Linden et al., 2003; Duvoisin et al., 2005, 2011), whereas others find an anxiolytic-like one (Gerlai et al., 2002; Fendt et al., 2010, 2013). Also, mGlu ${ }^{-1-}$ mice present robust deficits in contextual fear conditioning, novel object recognition, extinction of operant conditioning, and acoustic startle response (Fendt et al., 2010, 2013). $\mathrm{mGlu}_{8}^{-1-}$ mice also show enhanced social interaction; however, enhancing $\mathrm{mGlu}_{8}$ receptor activity does not affect social interaction in wild-type mice (Duvoisin et al., 2011). Further studies are required to better understand the role of $\mathrm{mGlu}_{8}$ and to clarify its therapeutic potential.

Outside of the CNS, the $\mathrm{mGlu}_{6}$ receptor plays an important role in visual discrimination in low light conditions (Nomura et al., 1994; Vardi et al., 2000), supported by the $\mathrm{mGlu}_{6}^{-1-}$ phenotype (Masu et al., 1995). Mutations in proteins involved in the transmission of the signal between rod photoreceptors and bipolar ON cells have been found in patients suffering from congenital stationary night blindness; these include more than 20 loss-of-function mutations in GRM6 (Dryja et al., 2005; O'Connor et al., 2006; Zeitz et al., 2007,2015 ). These mutations affect the normal mGlu 6 receptor response to the glutamate released from the photoreceptors, thus impairing signal transmission. The use of an optogenetic tool consisting of a chimera between $\mathrm{mGlu}_{6}$ receptor and melanopsin receptor has been suggested as a potential approach to restore light sensitivity (van Wyk et al., 2015).

\section{Orthosteric Ligands}

\section{A. Definitions and Mode of Action}

By definition, orthosteric ligands act in the same binding pocket as the endogenous ligand, competing to either activate or inhibit mGlu receptor activity. Glutamate and surrogate orthosteric agonists bind in the cleft between the two VFT lobes (Fig. 1). Upon binding, orthosteric agonists stabilize the closed state of the VFT, leading to a change in the relative orientation such that the extracellular domain dimer changes from a "resting" (R) to an active (A) state (Bessis et al., 2002). On the contrary, orthosteric antagonists prevent the full closure of the VFT (Bessis et al., 2000, 2002; Kunishima et al., 2000; Tsuchiya et al., 2002). Based on crystal structures, the main conformations that define the inactive and active states of mGlu receptors are the resting state Roo where both VFTs are open, and the active states Aco or Acc where one or both VFTs are closed, respectively. The two lobes are distant in the resting state and become closer in the active state (Kunishima et al., 2000; Tsuchiya et al., 2002). The closure of one VFT (Aco) is sufficient to induce a functional response from the receptor, but the closure of both VFTs (Acc) is necessary to achieve full activation (Kniazeff et al., 2004).

\section{B. Selectivity}

The L-glutamate binding site is highly conserved among the mGlu receptor family, resulting in difficulties in identifying compounds with subtype selectivity. Indeed, glutamate binds to all mGlu receptors under 
a similar conformation, where the residues participating in direct interactions with its amino acid moiety are fully conserved as well as two residues that interact with the carboxylate moiety (Bertrand et al., 2002; Acher and Bertrand, 2005; Wellendorph and BraunerOsborne, 2009). Within the orthosteric pocket, several residues that do not directly interact with glutamate are different between the three groups of mGlu receptors, enabling identification of group I, group II, and group III selective ligands (Table 1).

The most commonly used agonists of group I mGlu receptors are 3,5-dihydroxyphenylglycine (DHPG) and quisqualic acid, which are somewhat selective for group I over group II and III receptors (Table 1). Concerning group II mGlu receptors, the classic agonists are ( $2 S, 2^{\prime} R, 3^{\prime} R$ )-2-(2',3'-dicarboxycyclopropyl)glycine (DCG-IV) (Brabet et al., 1998) and (1S,2S,5R,6S)-2-amino-bicyclo [3.1.0]hexane-2,6-dicarboxylic acid (LY354740) (Monn et al., 1997), whereas L-AP4 and ACPT-I (Acher et al., 1997) are most commonly used agonists for group III mGlu receptors (Fig. 6). LY341495 is the most used competitive antagonist, which antagonizes all the subtypes, with higher potency at $\mathrm{mGlu}_{2 / 3}$ receptors over other subtypes (Kingston et al., 1998) (Fig. 6).

For subtype-selective orthosteric ligands, drug designers have to circumvent the highly conserved binding pocket. One way is to design compounds able to interact with proximal residues to the glutamate binding pocket that differ between subtypes. By example, newly discovered orthosteric ligands can discriminate between $\mathrm{mGlu}_{2}$ and $\mathrm{mGlu}_{3}$ receptors, for example, $(1 S, 2 S, 4 R, 5 R, 6 S)-2$ amino-4-methylbicyclo[3.1.0] hexane2,6-dicarboxylic acid and $(1 R, 2 S, 4 R, 5 R, 6 R)$-2-amino-4-(1H-1,2,4-triazol-3ylsulfanyl)bicyclo[3.1.0] hexane-2,6-dicarboxylic acid, are two $\mathrm{mGlu}_{2}$ receptor-selective agonists (Monn et al., $2015 \mathrm{a}, \mathrm{b})$, and (1S,2S,4S,5R,6S)-2-amino-4-[(3-methoxybenzoyl)amino]bicyclo[3.1.0]hexane-2,6-dicarboxylic acid is an $\mathrm{mGlu}_{3}$ receptor-selective agonist (Monn et al., 2018). Cocrystallization of the VFT with each of these ligands, coupled with mutagenesis and molecular modeling, revealed that selectivity is due to interactions with amino acids residing at the periphery of the glutamate binding site (Monn et al., 2015a,b, 2018). In a similar fashion, (2S)-2-amino-4-(\{[4-(carboxymethoxy)phenyl](hydroxy)methyl)(hydroxy)phosphoryl)butanoic acid (LSP42022), an $\mathrm{mGlu}_{4}$ receptor-selective orthosteric agonist, binds both to the glutamate binding site and to an adjacent pocket (Goudet et al., 2012) (Fig. 6). This adjacent pocket is thought to be one of the sites of action of $\mathrm{Cl}^{-}$ions, which are potent positive allosteric modulators of mGlu receptors (Acher et al., 2011; Tora et al., 2015). Therefore, LSP4-2022 and related derivatives constitute bitopic ligands that simultaneously target an orthosteric and an allosteric binding site (Selvam et al., 2018). Targeting these two sites in tandem provides the means to overcome the difficulty of designing selective orthosteric drugs.

\section{Biased Agonism}

Across the GPCR superfamily, it is becoming increasingly appreciated that the cellular response to receptor activation can differ depending on the ligand used, a phenomenon referred to as biased agonism. Biased agonism is thought to originate from the stabilization of different active conformations by distinct ligands, the balance of which is sampled by measuring different downstream measures of receptor activity. For any definition of biased GPCR agonism, it is critical that biased agonism is quantified relative to both a reference agonist and a reference pathway; this is because the relative efficacy of GPCR agonists is influenced by the stimulus-response coupling of the system. The most commonly applied method to quantify GPCR biased agonism is based on the operational model of agonism (Black and Leff, 1983) and subsequent derivation of transduction ratios (Kenakin et al., 2012). For metabotropic glutamate receptor orthosteric agonists, to date observations of biased agonism are limited to group I receptors. For example, relative to glutamate activation of $\mathrm{mGlu}_{1}$-mediated cytoprotective signaling, quisqualate is biased toward inositol monophosphate $\left(\mathrm{IP}_{1}\right)$ accumulation in recombinant and native cells (Emery

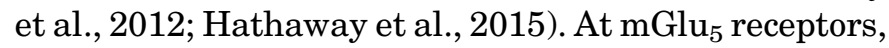
biased agonism for DHPG relative to glutamate arises due to "location bias" as DHPG is impermeable and not actively transported across cell membranes. DHPG is therefore unable to stimulate $\mathrm{mGlu}_{5}$ receptors located on intracellular membranes (Jong et al., 2005). Within different subcellular compartments, mGlu $_{5}$ receptors interact with a different complement of transducers, giving rise to different forms of synaptic plasticity (Kumar et al., 2012). It remains to be determined whether other mGlu receptors and associated selective ligands also exhibit location bias that contributes to pharmacological differences.

\section{Tolerance}

Another layer of complexity with regard to orthosteric agonist drug development is the potential for tolerance development. Under normal conditions glutamate is released transiently into the synapse, briefly activating mGlu receptors before active uptake mechanisms, for example, into astrocytes, reduce synaptic glutamate concentrations. However, these clearance mechanisms are not operative for surrogate orthosteric agonists, resulting in sustained receptor activation, which can lead to tolerance development. The potential for tolerance can be exacerbated for neurologic targets given the need for repeated chronic dosing to achieve a therapeutic effect. Tolerance has been noted for group II orthosteric agonists, where LY354740 efficacy for modulating rapid eye movement sleep wanes with repeated dosing (Ahnaou et al., 2015). Similarly, repeated daily dosing of (1R,4R,5S,6R)-4-amino-2-oxabicyclo[3.1.0] 
TABLE 1

Pharmacology of orthosteric metabotropic glutamate receptor agonists and antagonists

Commercially available agents. For a complete list refer to guidetopharmacology.org.

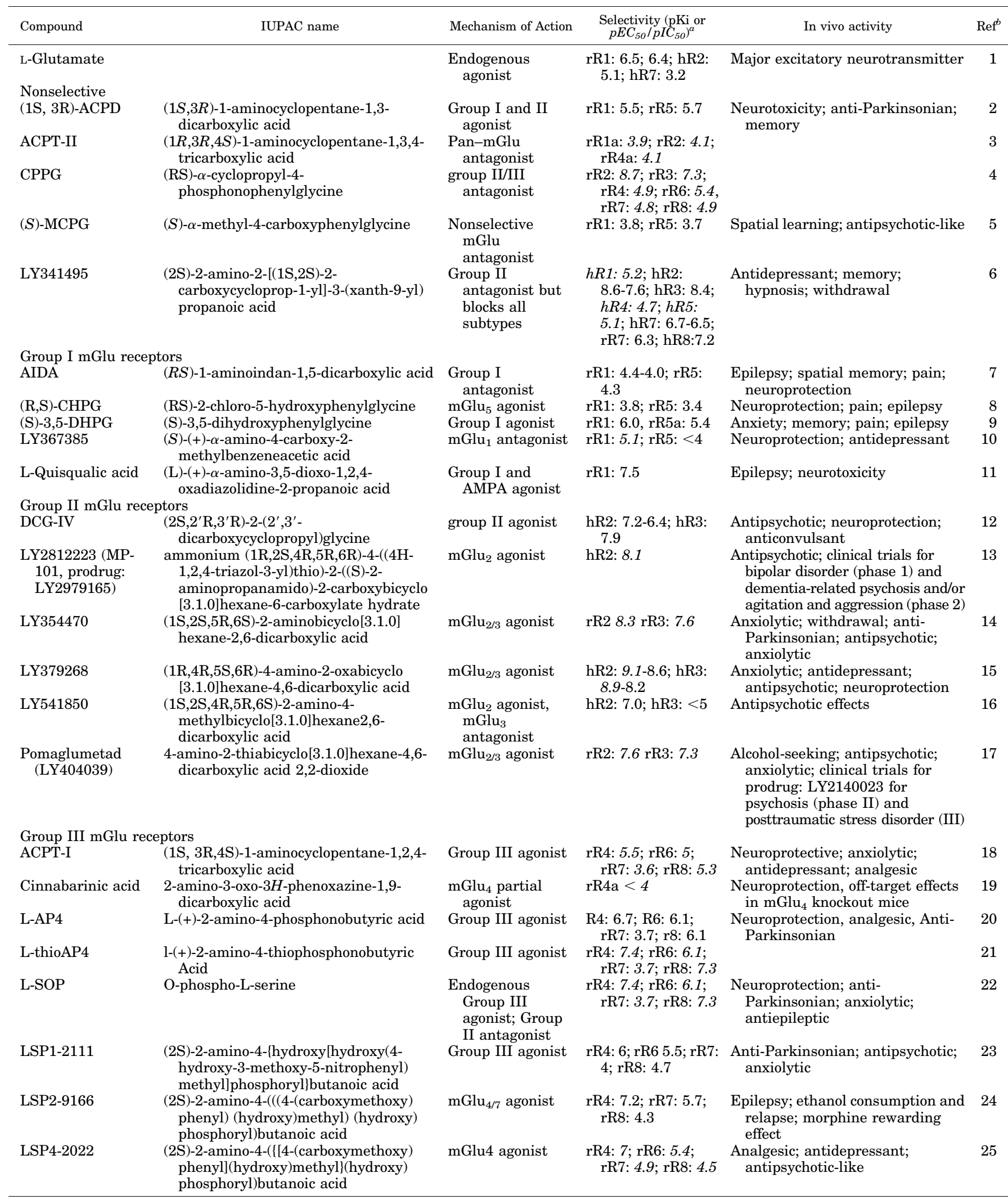


TABLE 1-Continued

\begin{tabular}{|c|c|c|c|c|c|}
\hline Compound & IUPAC name & Mechanism of Action & $\begin{array}{l}\text { Selectivity (pKi or } \\
\left.p E C_{50} / p I C_{50}\right)^{a}\end{array}$ & In vivo activity & $\operatorname{Ref}^{b}$ \\
\hline
\end{tabular}

IUPAC, International Union of Pure and Applied Chemistry; $\mathrm{pK}_{\mathrm{i}}$, negative logarithm of the equilibrium dissociation constant.

${ }^{a}$ Where affinity estimates were unavailable $\mathrm{pIC}_{50}$ (antagonists) or $\mathrm{pEC}_{50}$ (agonists) values are reported indicated by italics.

${ }^{b}$ 1) Pin et al., 1999; Meldrum, 2000; Mutel et al., 2000. 2) Zalewska and Wiśniewski, 1997; Henrich-Noack and Reymann, 1999; Mutel et al., 2000; Conti et al., 2002. 3 Acher

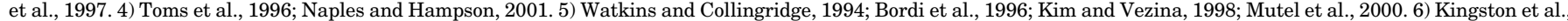

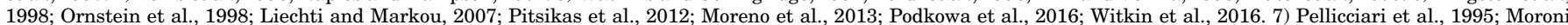

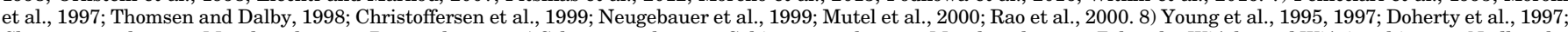

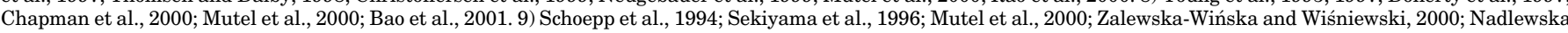

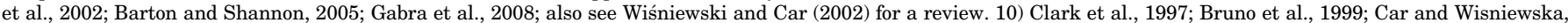

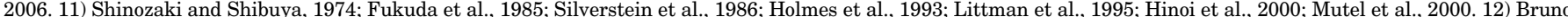

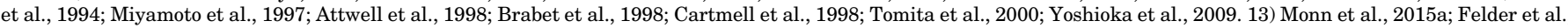

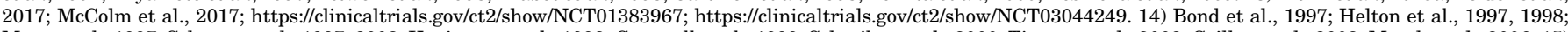

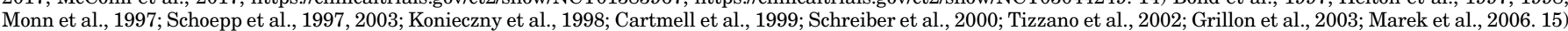

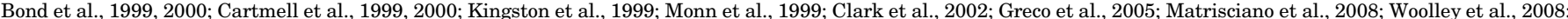

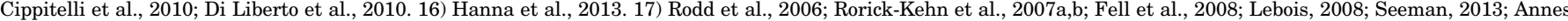

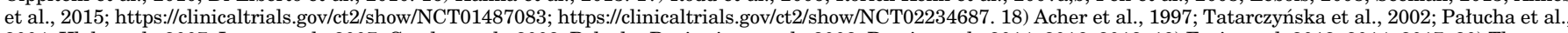

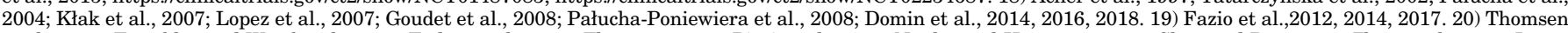

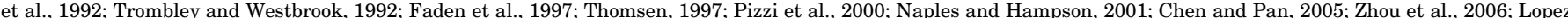

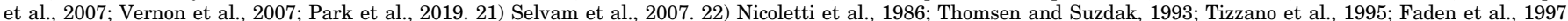

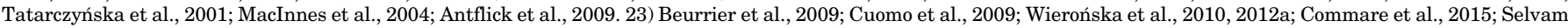

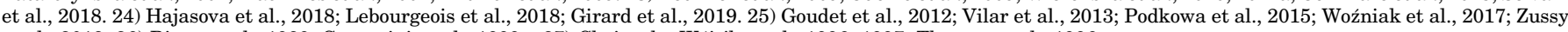
et al., 2018. 26) Bigge et al., 1989; Gasparini et al., 1999a. 27) Chojnacka-Wójcik et al., 1996, 1997; Thomas et al., 1996.

hexane-4,6-dicarboxylic acid (LY379268) results in loss of efficacy as an analgesic (Jones et al., 2005) and antipsychotic-like activity to inhibit phencyclidine- or amphetamine-induced hyperlocomotion (Galici et al., 2005). However, tolerance is not consistently observed with chronic LY379268 dosing and can differ between behavioral paradigms (Cartmell et al., 2000; Anderson et al., 2014; Battaglia et al., 2015; Halberstadt et al.,
2019). Whether tolerance development will prove to limit therapeutic efficacy of mGlu receptor orthosteric agonists remains to be seen.

\section{E. Orthosteric Ligands in the Clinic: Success and Failure}

The most successful mGlu receptor discovery campaigns focused on orthosteric ligands targeting group II<smiles>N[C@@H](CCC(=O)O)C(=O)O</smiles>

Glutamate<smiles>N[C@@H](C(=O)O)c1cc(O)cc(O)c1</smiles>

(S)-3,5-DHPG<smiles>N[C@@H](C(=O)O)n1oc(=O)[nH]c1=O</smiles>

Quisqualate<smiles>N[C@@H](C(=O)O)C1C(C(=O)O)[C@H]1C(=O)O</smiles>

DCG-IV<smiles>N[C@@H](CC[P+](=O)O)C(=O)O</smiles>

L-AP4<smiles>N[C@@]1(C(=O)O)CCC2C(C(=O)O)[C@H]21</smiles>

LY354740<smiles>NC1(C(=O)O)CC(C(=O)O)C(C(=O)O)C1</smiles>

ACPT-I<smiles>NC(CC1c2ccccc2Oc2ccccc21)(C(=O)O)C1CC1C(=O)O</smiles>

LY341495<smiles>CC(C)(C)S(C)(C)(C)(C)C</smiles>

LSP4-2022

Fig. 6. Structures of select orthosteric ligands of mGlu receptors. Representative orthosteric ligands for mGlu receptors. Antagonists are labeled with black text, and agonists are in blue. Detailed in vitro and in vivo pharmacological profiles are listed in Table 1. 
receptors. Multiple group II orthosteric agonists have reached phase II or phase III trials for psychiatric indications. LY354740/eglumegad was well tolerated and showed anxiolytic efficacy in humans (Grillon et al., 2003; Schoepp et al., 2003), with further development focused on a prodrug formulation (LY544344) to improve bioavailability (Rorick-Kehn et al., 2006). However, trials for generalized anxiety disorder were discontinued due to concerns regarding convulsions in animals (Dunayevich et al., 2008). Another group II mGlu receptor-selective agonist, LY2140023/pomaglumetad [prodrug for 4-amino-2-thiabicyclo[3.1.0] hexane-4,6-dicarboxylic acid 2,2-dioxide], improved both negative and positive symptoms in patients with schizophrenia in a randomized phase II clinical trial (Patil et al., 2007). Subsequent phase III trials failed to report significant antipsychotic efficacy for all the patients involved, terminating further development (Kinon et al., 2011; Adams et al., 2013, 2014; Downing et al., 2014). Interestingly, exploratory analyses of multiple phase II and III revealed that some subgroups of patients exhibited improvement after treatment with pomaglumetad, particularly early-indisease patients or individuals previously treated with $\mathrm{D}_{2}$ dopamine receptor-targeting drugs but not $5-\mathrm{HT}_{2}$ receptor antagonists (Kinon et al., 2015). Findings consistent with preclinical studies showing that $\mathrm{mGlu}_{2}$ and $5-\mathrm{HT}_{2 \mathrm{~A}}$ receptors form functional complexes in cortex and that atypical antipsychotic treatment downregulates $\mathrm{mGlu}_{2}$ receptor expression (González-Maeso et al., 2008; Kurita et al., 2012) (reviewed in Shah and Gonzalez-Maeso, 2019). Trials are ongoing for pomaglumetad for methamphetamine abuse [ClinicalTrials.gov identifier NCT03106571] and psychosis [NCT03321617]. Fasoracetam (also known as NS-105 or NFC-1), which is structurally unrelated to eglumegad or pomaglumegad, has agonist activity at group II and III receptors and showed efficacy for attention deficit hyperactivity disorder in a small cohort of adolescents (Elia et al., 2018), but failed to distinguish from placebo in a subsequent trial [NCT02777931]. In addition to orthosteric agonists, the group II selective orthosteric antagonist (1R,2R,3R,5R,6R)-2-amino-3-((3,4-dichlorobenzyl)oxy)6-fluorobicyclo[3.1.0] hexane-2,6-dicarboxylic acid (BCI-838; also known as MGS0210), a prodrug of (1R,2R,3R,5R,6R)2-amino-3-((3,4-dichlorobenzyl)oxy)-6-fluorobicyclo[3.1.0] hexane-2,6-dicarboxylic acid (BCI-632; also known as MGS0039) (Nakamura et al., 2006), completed phase I in healthy volunteers [NCT01546051], although plans for subsequent phase II trial in treatment resistant depression have not eventuated. This same agent has shown preclinical efficacy in models for Alzheimer's disease (Kim et al., 2014) and posttraumatic stress disorder related to traumatic brain injury (Perez-Garcia et al., 2018). Despite successful discovery efforts for subtype-selective and drug-like orthosteric ligands for the group II mGlu receptors in particular, the majority of mGlu receptor discovery programs are pursuing allosteric modulators as reviewed below.

\section{Allosteric Modulators}

\section{A. Definitions, Quantification, and Identification}

Since glutamate is recognized by two different receptor families (ionotropic and metabotropic receptors) as well as transporters, there remains a concern that orthosteric compounds will suffer from lack of selectivity due to high conservation of glutamate binding sites across different proteins. As such, many discovery programs have focused efforts on identification and development of allosteric modulators. Allosteric modulators interact with sites that are topographically distinct from the orthosteric site, such that a receptor may be simultaneously bound by both an orthosteric and an allosteric ligand (Fig. 1A). For the most part, allosteric sites are located in region of receptors that show greater sequence divergence across subtypes and therefore offer greater selectivity. An allosteric modulator may enhance or inhibit the binding and/or efficacy of an orthosteric ligand, with the magnitude and direction described as "cooperativity." An allosteric modulator that enhances orthosteric ligand affinity or efficacy is referred to as a positive allosteric modulator (PAM), whereas an inhibitor is a negative allosteric modulator (NAM). In addition, allosteric ligands may also bind to a receptor but have no net effect on either affinity or efficacy of an orthosteric ligand; referred to as neutral allosteric ligands (NALs). Furthermore, allosteric ligands may also possess intrinsic efficacy as either positive or inverse agonists in addition to, or exclusive of, cooperativity with an orthosteric ligand. By example, a PAM with intrinsic agonist activity is referred to as a PAM agonist or ago-PAM.

In addition to potential for increased subtype selectivity, allosteric modulators offer a number of advantages over their orthosteric counterparts. Cooperativity between two ligands is saturable, offering the potential for greater safety in an overdose. Allosteric modulators that have no intrinsic efficacy and are quiescent in the absence of endogenous ligand also provide scope to fine-tune receptor activity in a spatiotemporal fashion, exerting potentiation or inhibition only where, and when, the endogenous ligand is present. For these reasons, discovery programs in industry and academia alike have sought allosteric modulators of mGlu receptors as potential novel therapeutics for a wide array of CNS disorders. However, discovery of allosteric modulators can be associated with considerable challenges with respect to quantification and validation.

Allosteric modulator binding is defined by the law of mass action, where the equilibrium dissociation constant, commonly defined as $\mathrm{K}_{\mathrm{B}}$, describes the affinity of 
an allosteric modulator for its site. However, the simultaneous binding of an allosteric modulator and orthosteric ligand gives rise to different receptor conformations than can be achieved by the binding of each ligand individually, altering ligand affinity as defined by cooperativity. To quantify cooperativity, the simplest scheme is the allosteric ternary complex model (ATCM) (Fig. 1B; Gregory et al., 2010b), which describes the reciprocal change in ligand affinity when a receptor is simultaneously bound by both an allosteric and orthosteric ligand, defined by the cooperativity factor $\alpha$. The ATCM is limited to describing allosteric interactions at the level of receptor binding, and for many mGlu allosteric modulators it is apparent these ligands have effects on receptor activity in addition to, or independent of, affinity.

To quantify the full scope of effects an allosteric ligand may have on receptor activity, multiple alternative pharmacological models have been proposed that can accommodate allosteric ligand intrinsic efficacy and efficacy modulation (Slack and Hall, 2012; Hall, 2013; Roche et al., 2014; Hall and Giraldo, 2018; Gregory et al., 2020). A challenge in applying these models is the inclusion of many parameters, which can prohibit fitting to experimental data. In this respect, the most widely adopted framework for quantification of pharmacological activity is an operational model of allosterism (Fig. 1C), which combines the Black and Leff operational model of agonism with the ATCM (Leach et al., 2007; Gregory et al., 2012). Within this framework, the influence of an allosteric modulator on orthosteric agonist efficacy is accounted for by $\beta$, an experimentally derived scaling factor. Application of this model therefore allows for delineation of the influence of an allosteric modulator on affinity independently of efficacy. This provides an important distinction given that allosteric modulators can have differential effects, which may be in opposing directions, on affinity versus efficacy. The operational model of allosterism also allows for intrinsic allosteric agonism, defined by $\tau$, but cannot account for inverse agonism.

Accurate quantification of allosteric ligand pharmacology requires appropriately designed experimental paradigms. The definitive experiment to unambiguously demonstrate an allosteric mechanism of action is a kinetic binding paradigm. The simultaneous binding of an allosteric ligand may enhance or slow the dissociation rate $\left(\mathrm{K}_{\mathrm{off}}\right)$ of the orthosteric radioligand from the receptor, or vice versa. Interaction studies using radiolabeled orthosteric ligands can be used to quantify modulation of affinity $(\alpha)$ as well as ligand affinity for the free receptor (Gregory et al., 2010b). However, it is important to note that the magnitude and direction of cooperativity between two ligands depends on the chemotypes present, a phenomenon known as "probe dependence." This is an important consideration when extrapolating pharmacological profiles of allosteric ligands based on interactions with a radiolabeled orthosteric antagonist or from a surrogate orthosteric agonist, which is often required in native cells/tissues. Radiolabeled allosteric ligands have been described for multiple subtypes, which can also be used to quantify affinity for the receptor for unlabeled ligands at a common allosteric site (Cosford et al., 2003; Lavreysen et al., 2003; O'Brien et al., 2018) or provide evidence for additional allosteric sites that are conformationally linked such that there is cooperativity between the two allosteric sites.

The vast majority of allosteric ligands for mGlu receptors have been identified and validated using functional assays. The most commonly used approach involves generating modulator titration curves in the presence of either an $\sim \mathrm{EC}_{20}$ agonist concentration for PAM identification, or an $\mathrm{EC}_{80}$ to identify NAMs. The potencies and relative maximum response (for PAMs) or inhibitory effect (for NAMs) from these titration curves are routinely used to drive discovery programs (Lindsley et al., 2016). However, these parameters represent composite values encompassing $\alpha, \beta, \mathrm{K}_{\mathrm{B}}$, and $\tau$ and are also influenced by the concentrations of orthosteric agonist used, orthosteric agonist intrinsic efficacy, and the stimulus-response coupling of the system under investigation (Lindsley et al., 2016). Modulator potencies curves can be analyzed in parallel with an agonist concentration-response curve to estimate $\mathrm{K}_{\mathrm{B}}$ and a composite $\alpha \beta$ value where the maximum degree of potentiation or inhibition does not reach the limit of the system (Gregory et al., 2012, 2019). However, to quantify the interaction between a modulator and orthosteric agonist, the most robust approach is to perform full agonist concentration-response curves in the absence and presence of increasing concentrations of modulator. Despite the limitations in the most commonly applied screening approaches, drug discovery programs for small molecule synthetic allosteric ligands of mGlu receptors have been largely successful. In addition, there is increasing evidence for endogenous allosteric modulators for mGlu receptor family members.

\section{B. Endogenous Allosteric Modulators}

The greater class C GPCR family also includes the calcium-sensing receptor and GPRC6A, two receptors that are known to respond to multiple endogenous ligands including amino acids and cations (Leach and Gregory, 2017). It is perhaps therefore not surprising that divalent and trivalent cations, including $\mathrm{Ca}^{2+}$, can directly activate $\mathrm{mGlu}_{1}, \mathrm{mGlu}_{3}$, and $\mathrm{mGlu}_{5}$ receptors (Kubo et al., 1998; Miyashita and Kubo, 2000a,b; Jiang et al., 2014). Furthermore, extracellular $\mathrm{Ca}^{2+}$ potentiates binding/function of orthosteric ligands at $\mathrm{mGlu}_{1}$ (Saunders et al., 1998; Jiang et al., 2014). In addition, negatively charged chloride ions activate $\mathrm{mGlu}_{3}, \mathrm{mGlu}_{4}$, $\mathrm{mGlu}_{6}$, and $\mathrm{mGlu}_{8}$ receptors and potentiate glutamate 
efficacy at $\mathrm{mGlu}_{1}, \mathrm{mGlu}_{2}, \mathrm{mGlu}_{4}, \mathrm{mGlu}_{5}$, and $\mathrm{mGlu}_{6}$ receptors (DiRaddo et al., 2015; Tora et al., 2015, 2018). Both cations and anions are thought to mediate activation and/or modulation via interactions with the VFT domain. The extracellular membrane associated cellular prion protein interacts with the $\mathrm{mGlu}_{5}$ receptor acting as a coreceptor for amyloid $\beta$ oligomers, although the precise binding interactions within mGlu $_{5}$ receptors are unknown (Um et al., 2013). Beyond the extracellular domains, molecular dynamics studies have proposed that lipids can interact with $\mathrm{mGlu}_{5}$ 7TM (Dalton et al., 2017). Furthermore, cholesterol membrane content enhances $\mathrm{mGlu}_{1}$ signaling to ERK1/2 phosphorylation mediated via a cholesterol recognition/interaction amino acid consensus motif in transmembrane domain 5 (Kumari et al., 2013). The existence of endogenous allosteric modulators for the mGlu receptors is often overlooked during discovery and validation of synthetic small molecule allosteric modulators.

\section{Small Molecule Allosteric Modulators}

Concerted discovery efforts from both industrial and academic researchers have yielded a wealth of chemically and pharmacologically diverse small molecule allosteric modulators for the mGlu receptor family (Tables 2-5). The majority of small molecule mGlu receptor allosteric modulators identified to date interact with a common pocket within the 7TM domains. This binding pocket is in a location analogous to the biogenic amine orthosteric site of class A GPCRs, largely lined by residues in transmembrane domains $3,5,6$, and 7 . To date, six X-ray crystal structures of the $\mathrm{mGlu}_{1}$ or $\mathrm{mGlu}_{5}$ receptor 7TM domains have been solved with NAMs occupying this common allosteric site (Doré et al., 2014; Wu et al., 2014; Christopher et al., 2015, 2019). A wealth of previous mutagenesis data indicate that this pocket is shared across the mGlu receptor family, and indeed for all class C GPCRs, and can be engaged by both NAMs and PAMs (see Leach and Gregory, 2017, for review). Here we focus on the pharmacological profiles of prototypical and well validated commercially available allosteric modulators for mGlu receptors.

\section{Group I PAMs, NAMs, NALs}

The first disclosed mGlu receptor allosteric modulator was ethyl (7Z)-7-hydroxyimino-1,7a-dihydrocyclopropa[b]chromene-1a-carboxylate (CPCCOEt) (Annoura et al., 1996; Litschig et al., 1999), a negative allosteric modulator of $\mathrm{mGlu}_{1}$ receptor. CPCCOEt has low micromolar affinity for $\mathrm{mGlu}_{1}$ receptors (Lavreysen et al., 2003) and negatively modulates glutamate efficacy but has neutral cooperativity with respect to $\left[{ }^{3} \mathrm{H}\right]$ glutamate affinity (Litschig et al., 1999). Moreover, CPCCOEt has poor selectivity between group I mGlu receptors (Table 2), negatively modulating $\mathrm{mGlu}_{5}$ receptor activation with a similar apparent $\mathrm{K}_{\mathrm{B}}$ (Hellyer et al., 2018).
The discovery of CPCCOEt was followed by EM-TBPC and BAY-36-7620, which showed species differences in $\mathrm{mGlu}_{1}$ receptor NAM activity with considerably higher affinity for the rat versus human receptor (Malherbe et al., 2003; Cho et al., 2014a). Similar to CPCCOEt, BAY-36-7620 has neutral cooperativity with respect to $\left[{ }^{3} \mathrm{H}\right]$ quisqualate affinity but inhibits orthosteric agonist efficacy (Carroll et al., 2001; Lavreysen et al., 2003). Since the discovery of these early tool compounds, a wealth of structurally diverse mGlu receptor NAMs have been disclosed that have therapeutic efficacy in preclinical models for analgesia, antipsychotic-like activity, anxiety, addiction, and cancer and as anticonvulsants (Table 2). For diverse scaffolds [Fig. 7, e.g., A-841720 and 1-(3,4-dihydro-2H-pyrano[2,3-b] quinolin-7-yl)-2-phenylethanone], the higher affinity for rat over human (>10-fold) persisted (Cho et al., 2014a). Breakthrough chemotypes represented by FTIDC and JNJ16259685 have similar nanomolar affinities for the rat and human receptors and $>100$-fold selectivity as NAMs for $\mathrm{mGlu}_{1}$ over $\mathrm{mGlu}_{5}$ receptors (Lavreysen et al., 2003, 2004; Suzuki et al., 2007a). Despite ultimate identification of high affinity, in vivo efficacious $\mathrm{mGlu}_{1}$ receptor NAMs, further development has stalled due to on-target mediated adverse effects such as cognitive impairments from multiple scaffolds (Steckler et al., 2005b; Schröder et al., 2008).

On the other hand, $\mathrm{mGlu}_{1}$ receptor PAMs have been relatively unexplored, although they may be a promising therapeutic strategy for schizophrenia by restoring function of naturally occurring mutations (GarciaBarrantes et al., 2015b). The first $\mathrm{mGlu}_{1}$ receptor PAMs included diverse chemotypes, for example, 2-(4-fluorophenyl)-1-(4-methylphenyl)sulfonylpyrrolidine (RO 677476) and ethyl $\mathrm{N}$-[2,2-di(phenyl)acetyl]carbamate, identified from high-throughput screening, which enhanced orthosteric agonist affinity and functional responses at rat $\mathrm{mGlu}_{1}$ receptor without intrinsic agonist activity (Knoflach et al., 2001) but were not suitable for in vivo studies. Similar to multiple $\mathrm{mGlu}_{1}$ receptor NAM scaffolds, RO 67-7476 lacks the ability to potentiate glutamate at human $\mathrm{mGlu}_{1}$ receptors (Knoflach et al., 2001). A subsequent study suggested these $\mathrm{mGlu}_{1}$ receptor PAMs may have intrinsic efficacy for ERK1/2 and cAMP accumulation; however, this agonist activity could be blocked by both orthosteric and allosteric antagonists, raising the possibility that the apparent intrinsic agonism may be attributable to potentiation of ambient glutamate (Sheffler and Conn, 2008). Of note, both RO 67-7476 and ethyl N-[2,2-di(phenyl) acetyl]carbamate were unable to completely displace binding of the radiolabeled $m G u_{1}$ receptor NAM $\left[{ }^{3} \mathrm{H}\right] 1$ (3,4-dihydro-2H-pyrano[2,3-b]quinolin-7-yl)-2-phenylethanone (Hemstapat et al., 2006), suggesting these compounds recognize a different site within the 7TM domain. Subsequent discovery efforts identified 3-chloro-N-[3chloro-4-(4-chloro-1,3-dihydro-1,3-dioxo-2H-isoindol-2-yl) 
TABLE 2

Pharmacology of commercially available $\mathrm{mGlu}_{1}$ allosteric ligands

For a complete list refer to guidetopharmacology.org.

\begin{tabular}{|c|c|c|c|c|}
\hline Compound & IUPAC name & $\begin{array}{l}\text { Mechanism of } \\
\text { Action }\end{array}$ & $\begin{array}{l}\text { Selectivity }^{a}\left(\mathrm{pK}_{\mathrm{B}} \text { or }\right. \\
\left.\quad p E C_{50} / p I C_{50}\right)\end{array}$ & In vivo activity \\
\hline A-841720 & $\begin{array}{l}\text { 3-(azepan-1-yl)-9-(dimethylamino)pyrido } \\
\text { [1,2] thieno[3,4-d]pyrimidin-4-one }\end{array}$ & NAM & $\begin{array}{l}\text { rR1: } 9.0 ; \text { hR1: } 8.0 \\
\quad \text { rR5: } 6.7\end{array}$ & Analgesic; disrupts locomotion and cognition \\
\hline BAY-36-7620 & $\begin{array}{l}\text { [(3aS,6aS)- 6a-naphtalen-2-ylmethyl-5- } \\
\text { methyliden-hexahydro-cyclopental[c] } \\
\text { furan-1-on] }\end{array}$ & NAM & rR1: $8.0 ; \mathrm{hR} 1:<5$ & Anticonvulsive, cognitive impairments \\
\hline CFMTI & $\begin{array}{l}\text { 2-cyclopropyl-5-[1-(2-fluoropyridin-3-yl)- } \\
\text { 5-methyltriazol-4-yl]-3H-isoindol-1-one }\end{array}$ & NAM & $\begin{array}{l}\text { hR1: } 8.6 ; \text { rR1: } 8.6 \\
\text { hR5: } 5.3\end{array}$ & Antipsychotic-like; no motor effects \\
\hline CPCCOEt & $\begin{array}{l}\text { ethyl (7Z)-7-hydroxyimino-1,7a- } \\
\text { dihydrocyclopropa[b]chromene-1a- } \\
\text { carboxylate }\end{array}$ & NAM & $\begin{array}{l}\text { rR1: } 5.3-4.9 ; \text { hR1: } \\
\text { 4.8; rR5: } 4.9 ; \\
\text { hR5: } 4.4 ; \mathrm{R} 4:<4\end{array}$ & $\begin{array}{l}\text { Antitumorigenic (melanoma), analgesia, } \\
\text { memory impairment, reverses morphine } \\
\text { tolerance; neuroprotective (trauma) }\end{array}$ \\
\hline $\begin{array}{l}\text { DFMTI (MK- } \\
\text { 5435) }\end{array}$ & $\begin{array}{l}\text { 5-(1-(2,4-difluorophenyl)-5-methyl- } 1 H \text { - } \\
\text { 1,2,3-triazol-4-yl)-2- } \\
\text { isopropylisoindolin-1-one }\end{array}$ & NAM & $\begin{array}{l}\text { rR1: 8.4-8.1; hR1: } \\
\quad \text { 8.4-7.5; hR5: } 5.8\end{array}$ & \\
\hline DM-PPP & $\begin{array}{l}\text { 4-O-[(2S)-3,3-dimethylbutan-2-yl] 2-O- } \\
\text { propyl 3,5-dimethyl-1H-pyrrole-2,4- } \\
\text { dicarboxylate }\end{array}$ & NAM & rR1: 7.8 & Analgesic \\
\hline EM-TBPC & $\begin{array}{l}\text { 1-ethyl-2-methyl-6-oxo-4-(1,2,4,5- } \\
\text { tetrahydro-3-benzazepin-3-yl) } \\
\text { pyrimidine-5-carbonitrile }\end{array}$ & NAM & rR1: 8.2; hR1: low & Not suitable for in vivo dosing \\
\hline $\begin{array}{l}\text { FITM, }\left[{ }^{18} \mathrm{~F}\right] \\
\text { FITM }\end{array}$ & $\begin{array}{l}\text { 4-fluoro-N-methyl-N-[4-[6-(propan-2- } \\
\text { ylamino)pyrimidin-4-yl]-1,3-thiazol-2- } \\
\text { yl]benzamide }\end{array}$ & NAM & $\begin{array}{l}\text { hR1: } 6.6 ; \mathrm{rR} 1: 8.7 \\
\quad \text { R5: } 5.2\end{array}$ & \\
\hline FPTQ & $\begin{array}{l}\text { 6-(1-(2-fluoropyridin-3-yl)-5-methyl- } 1 H \text { - } \\
\text { 1,2,3-triazol-4-yl)quinoline }\end{array}$ & NAM & hR1: 7.9 & \\
\hline FTIDC & $\begin{array}{l}\text { 4-[1-(2-fluoropyridin-3-yl)-5- } \\
\text { methyltriazol-4-yl]-N-methyl-N- } \\
\text { propan-2-yl-3,6-dihydro-2H-pyridine- } \\
\text { 1-carboxamide }\end{array}$ & $\begin{array}{l}\text { NAM } \\
\text { inverse } \\
\text { agonist }\end{array}$ & $\begin{array}{l}\mathrm{hR} 1: 8.2-8 ; \mathrm{mR} 1: \\
\quad 8.5 ; \mathrm{hR} 5: 5.2 \\
\quad \text { rR5: } 5\end{array}$ & \\
\hline JNJ16259685 & $\begin{array}{l}\text { 3,4-dihydro-2H-pyrano[2,3-b]quinolin-7- } \\
\text { yl-(4-methoxycyclohexyl)methanone }\end{array}$ & $\begin{array}{l}\text { NAM } \\
\text { inverse } \\
\text { agonist }\end{array}$ & $\begin{array}{l}\text { hR1: } 7.7-8.9 ; \mathrm{rR} 1: \\
\quad 8.3 ; \text { hR5: } 5.8-4.5\end{array}$ & $\begin{array}{l}\text { Antipsychotic-like, antiabuse/addiction, } \\
\text { anxiolytic, cognitive impairment, no } \\
\text { tolerance with repeat dosing, protective in } \\
\text { retinal neurodegeneration }\end{array}$ \\
\hline LY456066 & $\begin{array}{l}\text { 2-[[4-(2,3-dihydro-1H-inden-2-ylamino)- } \\
\text { 5,6,7,8-tetrahydroquinazolin-2-yl] } \\
\text { sulfanyl]ethanol }\end{array}$ & NAM & hR1: $7.7 ;$ hR5: < 5 & \\
\hline LY456236 & $\begin{array}{l}\text { 6-methoxy-N-(4-methoxyphenyl) } \\
\text { quinazolin-4-amine hydrochloride }\end{array}$ & NAM & $\begin{array}{l}\mathrm{rR} 1: 5.9 ; \mathrm{hR} 1: 6.9- \\
\quad 5.5\end{array}$ & Analgesic, anticonvulsant \\
\hline $\begin{array}{l}\mathrm{R} 214127,\left[{ }^{3} \mathrm{H}\right] \\
\quad \mathrm{R} 214127\end{array}$ & $\begin{array}{l}\text { 1-(3,4-dihydro-2H-pyrano[2,3-b] } \\
\text { quinolin-7-yl)-2-phenylethanone }\end{array}$ & NAM & $\begin{array}{l}\mathrm{rR} 1: 8.9-8.6 ; \mathrm{hR} 1: \\
\quad 7.5\end{array}$ & \\
\hline RO $67-7476$ & $\begin{array}{l}\text { 2-(4-fluorophenyl)-1-(4-methylphenyl) } \\
\text { sulfonylpyrrolidine }\end{array}$ & PAM & $\begin{array}{l}\text { rR1: } 6.8-6.7 ; \text { hR1: } \\
\quad<5\end{array}$ & \\
\hline RO 01-6128 & ethyl N-[2,2-di(phenyl)acetyl]carbamate & PAM & $\begin{array}{l}\text { rR1: } 6.7-6.6 ; \text { hR1: } \\
\quad<5\end{array}$ & \\
\hline VU0483605 & $\begin{array}{l}\text { 3-chloro-N-[3-chloro-4-(4-chloro-1,3- } \\
\text { dihydro-1,3-dioxo-2H-isoindol-2-yl) } \\
\text { phenyl]-2-pyridinecarboxamide }\end{array}$ & PAM & $\begin{array}{l}\text { hR1: } 6.4 ; \mathrm{rR} 1: 6.0 \\
\text { hR4: }<5 ; \\
\text { rR5(NAL): } 6.5\end{array}$ & \\
\hline YM298198 & $\begin{array}{l}\text { 6-amino-N-cyclohexyl-N,3- } \\
\text { dimethylthiazolo[3,2-a]benzimidazole- } \\
\text { 2-carboxamide }\end{array}$ & NAM & $\begin{array}{l}\text { hR1: } 6.9 ; \text { rR1: } 7.7 \\
\quad \text { hR5: }<5.2\end{array}$ & $\begin{array}{l}\text { Antipsychotic-like; analgesic, no motor } \\
\text { impairments }\end{array}$ \\
\hline
\end{tabular}

IUPAC, International Union of Pure and Applied Chemistry; MoA, mechanism of action; $\mathrm{pK}_{\mathrm{B}}$, negative logarithm of the equilbrium dissociation constant for an allosteric

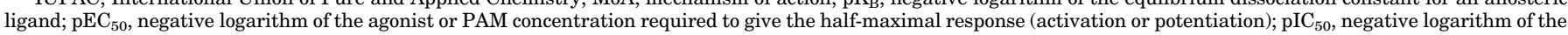
NAM concentration required to give the half-maximal inhibition.

${ }^{a}$ Where affinity estimates were unavailable $\mathrm{pIC}_{50}$ (NAMs) or $\mathrm{pEC}_{50}(\mathrm{PAMs})$ values are reported indicated by italics.

${ }^{b}$ 1) Zheng et al., 2005; El-Kouhen et al., 2006; Morè et al., 2007; Zhu et al., 2008. 2) Carroll et al., 2001; De Vry et al., 2001; Lavreysen et al., 2003; Schröder et al., 2008; Cho

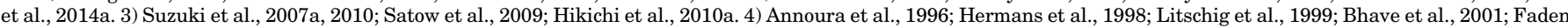

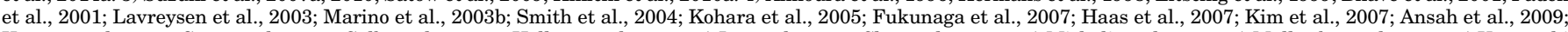

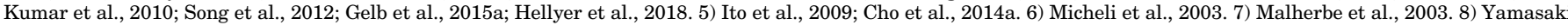

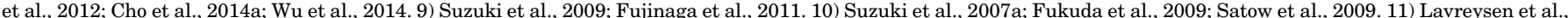

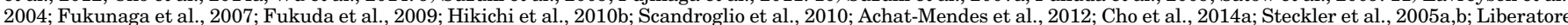

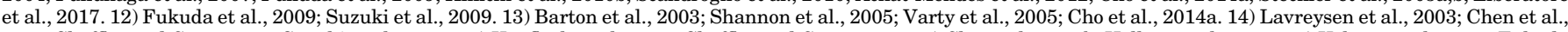

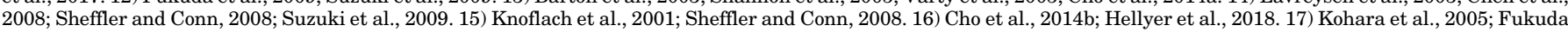
et al., 2009; Suzuki et al., 2009; Hikichi et al., 2010b; Scandroglio et al., 2010.

phenyl]-2-pyridinecarboxamide (VU0483605) based on a scaffold hop from an $\mathrm{mGlu}_{4} \mathrm{PAM} / \mathrm{mGlu}_{1}$ NAM chemotype (Cho et al., 2014b); however, the selectivity of VU0483605 as an mGlu ${ }_{1}$ PAM is based on cooperativity, as it has similar affinity for $\mathrm{mGlu}_{5}$ receptors, albeit with neutral cooperativity with $\mathrm{mGlu}_{5}$ receptor orthosteric agonist efficacy (Hellyer et al., 2018). Recent medicinal chemistry efforts have yielded the first CNS penetrant $\mathrm{mGlu}_{1}$ receptor PAMs (Garcia-Barrantes et al., 2015a, 2016a,b; Yohn et al., 2018), paving the way forward to establish therapeutic potential for schizophrenia and beyond.

Although CPCCOEt was the first mGlu receptor NAM disclosed, the $\mathrm{mGlu}_{5}$ receptor NAM fenobam was discovered earlier (Itil et al., 1978), but its mechanism 
TABLE 3

Pharmacology of commercially available $\mathrm{mGlu}_{5}$ allosteric ligands

For a complete list refer to guidetopharmacology.org.

\begin{tabular}{|c|c|c|c|c|c|}
\hline Compound & IUPAC name & $\begin{array}{l}\text { Mechanism of } \\
\text { Action }\end{array}$ & $\begin{array}{l}\text { Selectivity }{ }^{a}\left(\mathrm{pK}_{\mathrm{B}} \text { or }\right. \\
\left.\quad p E C_{50} / p I C_{50}\right)\end{array}$ & In vivo activity (clinical data) & $\operatorname{Ref}^{b}$ \\
\hline ADX47273 & $\begin{array}{l}\text { (S)-(4-fluorophenyl)-(3-[3-(4-fluoro- } \\
\text { phenyl)-[1,2,4]-oxadiazol-5-yl] } \\
\text { piperidin-1-yl)methanone }\end{array}$ & PAM & rR5: 5.5-5.2; $h R 5: 7.1$ & Cognition enhancement, antipsychotic-like & 1 \\
\hline CDPPB & $\begin{array}{l}\text { 3-cyano- } N \text {-(1,3-diphenyl- } 1 H \text {-pyrazol-5- } \\
\text { yl)benzamide }\end{array}$ & PAM & rR5: 7.4-5.9; $h R 5: 7.1$ & $\begin{array}{l}\text { Antipsychotic-like, neuroprotective in } \\
\text { Alzheimer's disease and Huntington's } \\
\text { disease models, cognition enhancement, } \\
\text { promote addiction recovery, tolerance } \\
\text { development }\end{array}$ & 2 \\
\hline CPPHA & $\begin{array}{l}\mathrm{N} \text {-[4-chloro-2-(phthalimidomethyl) } \\
\text { phenyl]salicylamide }\end{array}$ & PAM & $\begin{array}{l}\mathrm{rR} 1: 5.5 ; \mathrm{rR} 5: 6.9-5.5 \\
\text { hR5: } 6.5-6.3 ; \text { NAM } \\
\text { at } h R 4: 4.9 ; \text { and } \\
\text { rR8: } 5.1\end{array}$ & Not suitable for in vivo use & 3 \\
\hline CTEP & $\begin{array}{l}\text { 2-chloro-4-((2,5-dimethyl-1-(4- } \\
\text { (trifluoromethoxy)phenyl)- } \\
1 H \text {-imidazol-4-yl)ethynyl)pyridine }\end{array}$ & $\begin{array}{l}\text { NAM } \\
\text { inverse } \\
\text { agonist }\end{array}$ & $\begin{array}{l}\text { hR5: } 7.8 ; \text { rR5: } 8.0 \\
\text { mR5: } 7.9 ; \mathrm{A}_{3} \mathrm{AR}: \\
\text { 5.6; L-type Ca } \\
\text { channel: } 5.6\end{array}$ & $\begin{array}{l}\text { Huntington's disease, chronic stress, } \\
\text { Alzheimer's disease, deficits in } \mathrm{FMR}^{-1-} \text {; } \\
\text { anxiolytic }\end{array}$ & 4 \\
\hline DFB & 3,3'-difluorobenzaldazine & PAM & hR5: 5.6 ; rR5: 5.5-5.3 & Cognition enhancement & 5 \\
\hline $\begin{array}{l}\text { Dipraglurant } \\
\text { (ADX48621) }\end{array}$ & $\begin{array}{l}\text { 6-fluoro-2-[4-(2-pyridinyl)-3-butyn-1- } \\
\text { yl]imidazo[1,2-a]pyridine }\end{array}$ & NAM & R5: 7.5 & Antidyskinesia; PD-LID & 6 \\
\hline $\begin{array}{l}\text { Fenobam }\left[{ }^{3} \mathrm{H}\right] \\
\text { fenobam }\end{array}$ & $\begin{array}{l}\text { [N-(3-chlorophenyl)-N'-(4,5-dihydro-1- } \\
\text { methyl-4-oxo-1H-imidazole-2-yl) } \\
\text { urea] }\end{array}$ & $\begin{array}{l}\text { NAM } \\
\text { inverse } \\
\text { agonist }\end{array}$ & $\begin{array}{l}\text { hR5: 7.4-7.3; rR5: } \\
\text { 7.5-7.2; } \mathrm{A}_{3} \mathrm{AR} \\
\text { MAO-B }\end{array}$ & $\begin{array}{l}\text { Analgesia, antiabuse/addiction (cocaine, } \\
\text { methamphetamine) but appetite/sucrose } \\
\text { effects, anxiolytic; autism spectrum } \\
\text { disorder behaviors (FMR } 1^{-7-} \text { ); cognitive } \\
\text { deficits in wild-type mice; psychoactive/ } \\
\text { stimulant }\end{array}$ & 7 \\
\hline LSN2463359 & $\begin{array}{l}\text { N-(1-methylethyl)-5-(pyridin-4- } \\
\text { ylethynyl)pyridine-2-carboxamide }\end{array}$ & PAM & rR5: $6.4 ; h R 5: 7.5$ & $\begin{array}{l}\text { Antipsychotic-like, wake- promoting, } \\
\text { cognition enhancement }\end{array}$ & 8 \\
\hline $\begin{array}{l}\text { Mavoglurant } \\
\quad(\mathrm{AFQ} 056)\end{array}$ & $\begin{array}{l}\text { methyl (3aR,4S,7aR)-4-hydroxy-4-[2- } \\
\text { (3-methylphenyl)ethynyl]- } \\
\text { 3,3a,5,6,7,7a-hexahydro-2H-indole- } \\
\text { 1-carboxylate }\end{array}$ & NAM & hR5: 8.3-7.6; rR5: 7.2 & $\begin{array}{l}\text { Autism spectrum disorder behaviors } \\
\text { (FMR1 } 1^{-/-} \text {), sleep-wake modulation, } \\
\text { GERD, PD-LID, chorea in Huntington's } \\
\text { disease (Reilmann) }\end{array}$ & 9 \\
\hline 5-MPEP & 5-methyl-2-(phenylethynyl)pyridine & NAL & rR5: $6.7-6.0$ & Not suitable for in vivo use & 10 \\
\hline M-5MPEP & $\begin{array}{l}\text { 2-[2-(3-methoxyphenyl)ethynyl]-5- } \\
\text { methylpyridine }\end{array}$ & NAM & rR5: 7.0-6.2 & $\begin{array}{l}\text { Antiaddiction/abuse (cocaine), anxiolytic, } \\
\text { antidepressive, no psychotomimetic-like } \\
\text { effects }\end{array}$ & 11 \\
\hline $\begin{array}{l}\mathrm{MPEP} /\left[{ }^{3} \mathrm{H}\right] \\
\mathrm{MPEP}\end{array}$ & 2-methyl-6-(phenylethynyl)-pyridine & $\begin{array}{l}\text { NAM } \\
\text { inverse } \\
\text { agonist }\end{array}$ & $\begin{array}{l}\text { hR5: 8.8-8.2; rR5: } \\
\quad 8.8-8.0\end{array}$ & $\begin{array}{l}\text { Autism spectrum disorder behaviors (VPA, } \\
\text { BTBR, ENU2 mouse models); cognitive } \\
\text { impairment; psychostimulant, analgesia, } \\
\text { enhances alcohol sedation/hypnosis; anti- } \\
\text { Parkinsonian; antiaddiction (alcohol, } \\
\text { cocaine), reverse morphine tolerance, PD- } \\
\text { LID, antiepileptic (as an adjunct), sleep } \\
\text { modulation }\end{array}$ & 12 \\
\hline MTEP & $\begin{array}{l}\text { 3-((2-methyl-4-thiazolyl)ethynyl) } \\
\text { pyridine }\end{array}$ & $\begin{array}{l}\text { NAM } \\
\text { inverse } \\
\text { agonist }\end{array}$ & $\begin{array}{l}\text { hR5: } 8.3-7.9 ; \text { rR5: } \\
\quad 8.3-7.6\end{array}$ & $\begin{array}{l}\text { Anti-obsessive compulsive disorder } \\
\left.\text { (Sapap } 3^{-1-}\right) \text {, anti-Parkinsonian and } \\
\text { neuroprotective; psychostimulant; } \\
\text { antiaddiction (methamphetamine, alcohol, } \\
\text { cocaine) }\end{array}$ & 13 \\
\hline $\begin{array}{l}\text { Raseglurant } \\
(\text { ADX10059) }\end{array}$ & $\begin{array}{l}\text { 2-[(3-fluorophenyl)ethynyl]-4,6- } \\
\text { dimethyl-3-pyridinamine }\end{array}$ & NAM & $\mathrm{R} 5: \sim 8.0$ & GERD, migraine & 14 \\
\hline VU29 & $\begin{array}{l}N \text {-(1,3-diphenyl-1 } H \text {-pyrazolo-5-yl)-4- } \\
\text { nitrobenzamide }\end{array}$ & PAM & $r R 5: 7.6-6.2$ & Cognition enhancement & 15 \\
\hline VU0357121 & $\begin{array}{l}\text { 4-butoxy- } N \text {-(2,4-difluorophenyl) } \\
\text { benzamide }\end{array}$ & PAM & rR5: 5.7 & Not suitable for in vivo dosing & 16 \\
\hline VU0360172 & $\begin{array}{l}N \text {-cyclobutyl-6-[2-(3-fluorophenyl) } \\
\text { ethynyl]-3-pyridinecarboxamide } \\
\text { hydrochloride }\end{array}$ & PAM & rR5: 7.0-6.6 & $\begin{array}{l}\text { Antiepileptic; neuroprotective; antipsychotic- } \\
\text { like }\end{array}$ & 17 \\
\hline
\end{tabular}


TABLE 3-Continued

\begin{tabular}{|c|c|c|c|c|c|}
\hline Compound & IUPAC name & $\begin{array}{l}\text { Mechanism of } \\
\text { Action }\end{array}$ & $\begin{array}{l}\text { Selectivity }^{a}\left(\mathrm{pK}_{\mathrm{B}} \text { or }\right. \\
\left.\quad p E C_{50} / p I C_{50}\right)\end{array}$ & In vivo activity (clinical data) & $\operatorname{Ref}^{b}$ \\
\hline $\begin{array}{l}\text { VU0409551 } \\
\text { (JNJ- } \\
\text { 46778212) }\end{array}$ & $\begin{array}{l}\text { [6,7-dihydro-2-(phenoxymethyl) } \\
\text { oxazolo[5,4-c]pyridin-5(4H)- } \\
\text { yl](fluorophenyl)methanone }\end{array}$ & PAM & $\begin{array}{l}\text { hR5: } 6.6-5.4 ; \text { rR5: } \\
\text { 7.1; MAO-B: } 5.2\end{array}$ & $\begin{array}{l}\text { Cognition in Huntington's disease context; } \\
\text { reverse deficits in serine racemase }-/- \\
\text { antipsychotic-like; cognition enhancement }\end{array}$ & 18 \\
\hline VU0409106 & $\begin{array}{l}\text { 3-fluoro- } N \text {-(4-methyl-2-thiazolyl)-5-(5- } \\
\text { pyrimidinyloxy)benzamide }\end{array}$ & $\begin{array}{l}\text { NAM } \\
\text { inverse } \\
\text { agonist }\end{array}$ & $R 5: 7.6-6.9$ & Anxiolytic & 19 \\
\hline
\end{tabular}

IUPAC, International Union of Pure and Applied Chemistry; MAO-B, monoamine oxidase B; $\mathrm{pK}_{\mathrm{B}}$, negative logarithm of the equilbrium dissociation constant for an

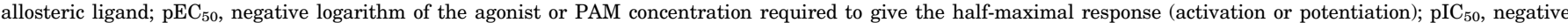
logarithm of the NAM concentration required to give the half-maximal inhibition.

${ }^{a}$ Where affinity estimates were unavailable $\mathrm{pIC}_{50}$ (NAMs) or $\mathrm{pEC}_{50}$ (PAMs) values are reported indicated by italics.

${ }^{b}$ 1) Xu et al., 2004; Liu et al., 2008; Bradley et al., 2011; Clifton et al., 2013; Gilmour et al., 2013; Ahnaou et al., 2015; Marszalek-Grabska et al., 2018. 2) Lindsley et al.,

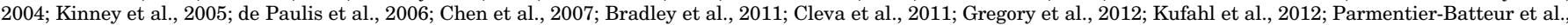

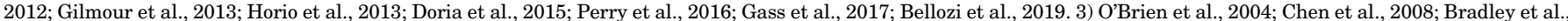

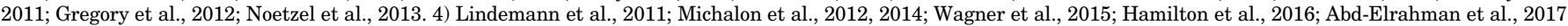

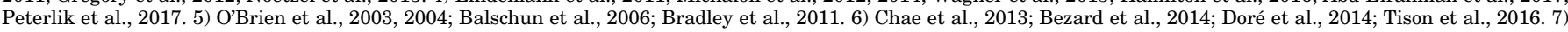

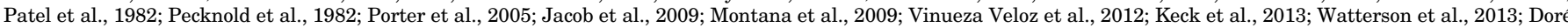

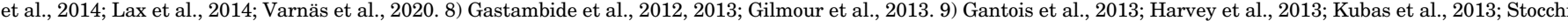

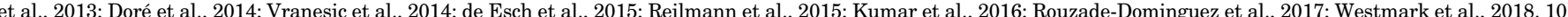

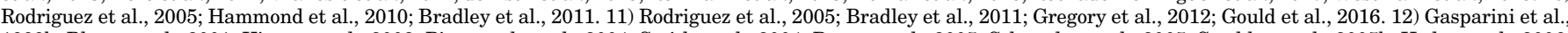

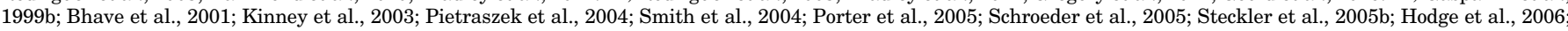

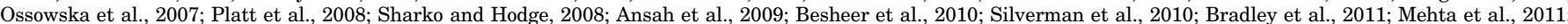

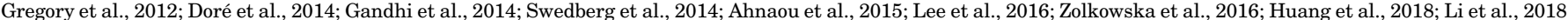

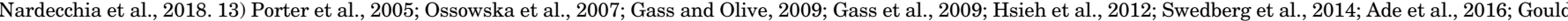

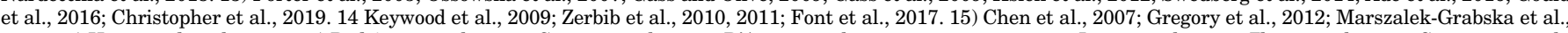

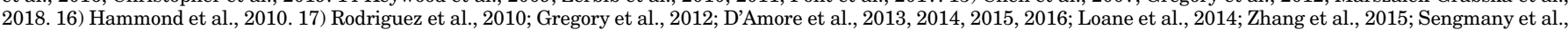
2017; Hanak et al., 2019. 18) Conde-Ceide et al., 2015; Rook et al., 2015b; Balu et al., 2016; Doria et al., 2018. 19) Felts et al., 2013; Rook et al., 2015a.

of action was not elucidated until over 20 years later (Porter et al., 2005). Indeed, of all the subtypes, allosteric ligand discovery against $\mathrm{mGlu}_{5}$ receptors has proven to be the most fruitful with a wealth of pharmacologically and structurally diverse ligands identified including NAMs, PAMs, and NALs (Table 3). Prototypical mGlu receptor $^{2}$ NAMs based on an acetylene core, 2-methyl-6-(phenylethynyl)-pyridine (MPEP) and 3-((2-methyl-4-thiazolyl) ethynyl)pyridine (MTEP), as well as fenobam, have demonstrated the therapeutic potential for $\mathrm{mGlu}_{5}$ receptor inhibition for addiction, depression, anxiety, neurodegenerative disorders, and autism spectrum disorders (Table 3). Often referred to as "full NAMs," these ligands have high negative cooperativity with respect to orthosteric agonist efficacy, completely abolishing agonist responses at saturating concentrations, but are neutral with respect to glutamate affinity (Gregory et al., 2012; Sengmany et al., 2019). The relatively high affinity of MPEP and fenobam presented the opportunity to generate radiolabeled versions (Cosford et al., 2003; Porter et al., 2005), which facilitated discovery and validation of novel $\mathrm{mGlu}_{5}$ receptor NAMs. Of note, many full NAMs have inverse agonist activity (Porter et al., 2005; Sengmany et al., 2019). It has been postulated that the combination of high negative cooperativity and inverse agonism contributes to on-target adverse effect liability of

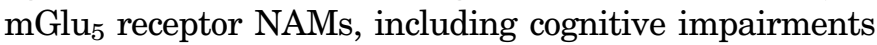
and psychotomimetic-like properties (Dekundy et al., 2011; Hughes et al., 2013; Abou Farha et al., 2014; Swedberg et al., 2014; Swedberg and Raboisson, 2014). Repeated exposure to both MTEP and fenobam is associated with tolerance development for reward behaviors (Cleva et al., 2012), but not for fenobam analgesic efficacy (Montana et al., 2011). The limitations associated with $\mathrm{mGlu}_{5}$ receptor full NAMs stimulated discovery efforts for
NAMs with lower negative cooperativity, also referred to as "partial NAMs," which have limited ability to inhibit glutamate efficacy. Two recent proof-of-concept studies demonstrated that partial NAMs, for example, 2-[2-(3methoxyphenyl)ethynyl]-5-methylpyridine (M-5MPEP) and N,N-diethyl-5-((3-fluorophenyl)ethynyl)picolinamide, which have limited negative cooperativity with glutamate, elicited anxiolytic, antidepressant activity and reduced cocaine self-administration with comparable efficacy to MTEP (Gould et al., 2016; Nickols et al., 2016). Importantly, unlike MTEP, M-5MPEP did not show psychotomimeticlike effects (Gould et al., 2016); therefore, $\mathrm{mGlu}_{5}$ receptor NAMs with limited cooperativity may offer improved therapeutic windows.

An inherent challenge for $\mathrm{mGlu}_{5}$ receptor allosteric ligand discovery has been the prevalence of "molecular switches" where minor substitutions give rise to ligands with reduced or opposing cooperativity (Wood et al., 2011). Although they pose a challenge with respect to structureactivity relationship interpretation, these molecular switches have also offered invaluable tools to dissect $\mathrm{mGlu}_{5}$ receptor biology, with the MPEP scaffold giving rise to NALs and PAMs. By example, 5MPEP is a neutral $\mathrm{mGlu}_{5}$ receptor allosteric ligand, which occupies the allosteric site in a competitive manner with MPEP but does not influence orthosteric agonist activity (Rodriguez et al., 2005). Subsequent efforts have identified high affinity $\mathrm{mGlu}_{5}$ receptor NALs [e.g., 3-azabicyclo[3.1.0] hexan-3yl-[5-[2-(3-fluorophenyl)ethynyl]pyridin-2-yl]methanone, (4R,5R)-rel-5-(2-chlorophenyl)-4-(5-(phenylethynyl)pyridin3 -yl)oxazolidin-2-one] with suitable properties for in vivo studies (Gregory et al., 2010a; Haas et al., 2017).

Molecular switches within mGlu $_{5}$ receptor NAM scaffolds (Fig. 7) have also yielded PAMs and PAM agonists, with advanced compounds from the biaryl 
TABLE 4

Pharmacology of commercially available group II mGlu allosteric ligands

For a complete list refer to guidetopharmacology.org.

\begin{tabular}{|c|c|c|c|c|c|}
\hline Compound & IUPAC name & $\begin{array}{l}\text { Mechanism of } \\
\text { Action }\end{array}$ & $\begin{array}{l}\text { Selectivity }^{a}\left(\mathrm{pK}_{\mathrm{B}} \text { or }\right. \\
\left.p E C_{50} / p I C_{50}\right)\end{array}$ & In vivo activity & $\operatorname{Ref}^{b}$ \\
\hline AZD8529 & $\begin{array}{l}\text { 7-methyl-5-[3-(piperazin-1-ylmethyl)- } \\
\text { 1,2,4-oxadiazol-5-yl]-2-[[4- } \\
\text { (trifluoromethoxy)phenyl]methyl]- } \\
\text { 3H-isoindol-1-one }\end{array}$ & R2 PAM & hR2: 6.4 & $\begin{array}{l}\text { Addiction (alcohol, nicotine, } \\
\text { methamphetamine) }\end{array}$ & 1 \\
\hline BINA & $\begin{array}{l}\text { 4-[3-[(2-cyclopentyl-6,7-dimethyl-1-oxo- } \\
\text { 2,3-dihydroinden-5-yl)oxymethyl] } \\
\text { phenyl]benzoic acid }\end{array}$ & $\begin{array}{l}\mathrm{R} 2 \mathrm{PAM} \\
\text { agonist }\end{array}$ & $\begin{array}{l}\text { hR2: } 8.4-6.2 ; r R 2: 7 \\
\quad \text { rR5(NAL): } 4.7\end{array}$ & $\begin{array}{l}\text { Anxiolytic, antipsychotic-like, sleep- } \\
\text { wake modulation, addiction (cocaine), } \\
\text { cognition, Sz negative symptoms, mania }\end{array}$ & 2 \\
\hline CBiPES & $\begin{array}{l}\mathrm{N} \text {-[4'-cyano-biphenyl-3-yl)-N-(3- } \\
\text { pyridinylmethyl)-ethanesulfonamide } \\
\text { hydrochloride) }\end{array}$ & R2 PAM & hR2: 7.0 & $\begin{array}{l}\text { Antipsychotic-like, locomotor effects, } \\
\text { antipanic }\end{array}$ & 3 \\
\hline $\begin{array}{l}\text { JNJ-46281222/ } \\
\quad\left[{ }^{3} \mathrm{H}\right]\end{array}$ & $\begin{array}{l}\text { 3-(cyclopropylmethyl)-7-[(4- } \\
\text { phenylpiperidin-1-yl)methyl]-8- } \\
\text { (trifluoromethyl)-[1,2,4]triazolo[4,3-a] } \\
\text { pyridine }\end{array}$ & R2 PAM & hR2: 8.8-8.3 & No reported in vivo activity & 4 \\
\hline LY2389575 & $\begin{array}{l}\text { (3S)-1-(5-bromopyrimidin-2-yl)-N-(2,4- } \\
\text { dichlorobenzyl) pyrrolidin-3-amine } \\
\text { methanesulfonate hydrate }\end{array}$ & R3 NAM & hR3: $6.7-5.4 ;$ hR2: 4.8 & No reported in vivo activity & 5 \\
\hline JNJ-42153605 & $\begin{array}{l}\text { 3-cyclopropylmethyl-7-(4- } \\
\text { phenylpiperidin-1-yl)-8- } \\
\text { trifluoromethyl[1,2,4]triazolo[4,3-a] } \\
\text { pyridine }\end{array}$ & $\begin{array}{l}\text { R2 PAM } \\
\text { agonist }\end{array}$ & hR2: $7.8-6.6$ & $\begin{array}{l}\text { Sleep/wake modulation, } \\
\text { antipsychotic-like, locomotor } \\
\text { effects, anticonvulsant }\end{array}$ & 6 \\
\hline $\begin{array}{l}\text { JNJ-40411813 } \\
\quad(\mathrm{ADX} 71149)\end{array}$ & $\begin{array}{l}\text { 1-butyl-3-chloro-4-(4-phenyl-1- } \\
\text { piperidinyl)-2(1H)-pyridinone }\end{array}$ & R2 PAM & $\begin{array}{l}\text { hR2: } 7.2-6.8 ; 5-\mathrm{HT}_{2 \mathrm{~A}} \\
\quad \text { (antag): } 6 \text { *metabolite } \\
\text { 5- } \mathrm{HT}_{2 \mathrm{~A}} \text { (antag): } 7\end{array}$ & $\begin{array}{l}\text { Antipsychotic-like, locomotor effects, } \\
\text { anticonvulsant, sleep modulation }\end{array}$ & 7 \\
\hline $\begin{array}{l}\text { LY487379 (4- } \\
\text { MPPTS) }\end{array}$ & $\begin{array}{l}\text { 2,2,2-trifluoro- } N \text {-[4-(2-methoxyphenoxy) } \\
\text { phenyl]- } N \text {-(3-pyridinylmethyl)- } \\
\text { ethanesulfonamide }\end{array}$ & R2 PAM & hR2: $7-6.3$ & $\begin{array}{l}\text { Anxiolytic, antipsychotic-like, } \\
\text { cognition }\end{array}$ & 8 \\
\hline $\begin{array}{l}\text { ML289 } \\
\quad \text { (VU0463597) }\end{array}$ & $\begin{array}{l}\text { [(3R)-3-(hydroxymethyl)-1-pipridinyl }][4- \\
{[2-(4 \text {-methoxyphenyl)ethynyl]phenyl] }} \\
\text { methanone }\end{array}$ & R3 NAM & hR3: $6.2-5.8$ & $\begin{array}{l}\text { Centrally active, no reported in vivo } \\
\text { efficacy }\end{array}$ & 9 \\
\hline ML337 & $\begin{array}{l}\text { [2-fluoro-4-[2-(4-methoxyphenyl) } \\
\text { ethynyl]phenyl][(3R)-3-hydroxy-1- } \\
\text { piperidinyl]methanone }\end{array}$ & R3 NAM & hR3: 7.1; rR5(NAL): 5.7 & No reported in vivo activity & 10 \\
\hline MNI-137 & $\begin{array}{l}\text { 4-(8-bromo-5-oxo-3,4,5,6-tetrahydro-1,6- } \\
\text { benzodiazocin-2-yl)pyridine-2- } \\
\text { carbonitrile }\end{array}$ & R2/3 NAM & $\begin{array}{l}\text { rR2: } 7.5-6.2 ; \text { hR2: } 8.1-7.1 \\
\text { rR3: } 7.7\end{array}$ & No reported in vivo activity & 11 \\
\hline Ro64-5229 & $\begin{array}{l}\text { (Z)-1-[2-cycloheptyloxy-2-(2,6- } \\
\text { dichlorophenyl)ethenyl]-1H-1,2,4- } \\
\text { triazole }\end{array}$ & $\begin{array}{r}\text { R2 NAM } \\
\text { inverse } \\
\text { agonist }\end{array}$ & rR2: 7.0 & No reported in vivo activity & 12 \\
\hline TASP0433864 & $\begin{array}{l}\text { (2S)-2-[[4-(1,1-dimethylethyl)phenoxy] } \\
\text { methyl]-2,3-dihydro-5-methylimidazo } \\
\text { [2,1-b]oxazole-6-carboxamide }\end{array}$ & R2 PAM & $\begin{array}{l}\text { rR2: } 7.1-6.7 ; \text { hR2: } 6.6 \\
\text { hR3: }<5 ; 5-\mathrm{HT}_{2 \mathrm{~B}}: 5.4 \\
\text { MAO-B: } 6.2\end{array}$ & Antipsychotic-like & 13 \\
\hline
\end{tabular}

IUPAC, International Union of Pure and Applied Chemistry; MAO-B, monoamine oxidase B; $\mathrm{pK}_{\mathrm{B}}$, negative logarithm of the equilbrium dissociation constant for an

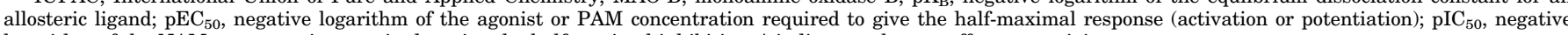
logarithm of the NAM concentration required to give the half-maximal inhibition; * indicates a known off target activity.

${ }^{a}$ Where affinity estimates were unavailable $\mathrm{pIC}_{50}(\mathrm{NAMs})$ or $\mathrm{pEC}_{50}(\mathrm{PAMs})$ values are reported indicated by italics.

${ }^{b}$ 1) Caprioli et al., 2015; Justinova et al., 2015; Augier et al., 2016; Li et al., 2016; Doornbos et al., 2017. 2) Galici et al., 2006; Benneyworth et al., 2007; Ahnaou et al., 2009;

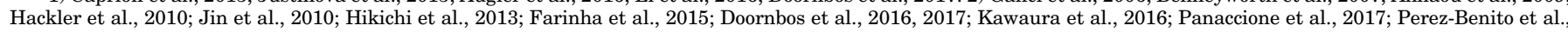

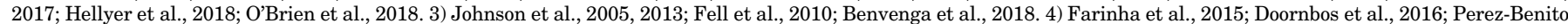

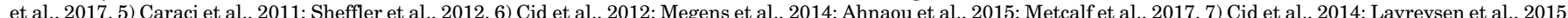

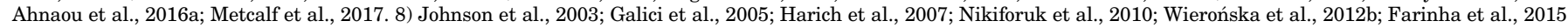

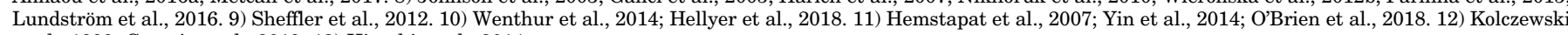
et al., 1999; Gutzeit et al., 2019. 13) Hiyoshi et al., 2014.

acetylene scaffold, such as $N$-cyclobutyl-6-[2-(3-fluorophenyl)ethynyl]-3-pyridinecarboxamide hydrochloride (VU0360172) and N-(1-methylethyl)-5-(pyridin-4-ylethynyl)pyridine-2-carboxamide, showing high affinity

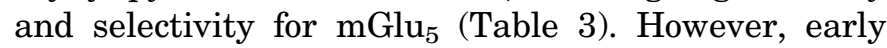
$\mathrm{mGlu}_{5}$ receptor PAMs [DFB, ADX47273, 3-cyano- $N$ (1,3-diphenyl- $1 H$-pyrazol-5-yl)benzamide (CDPPB), $N$ [4-chloro-2-(phthalimidomethyl)phenyl] salicylamide (CPPHA)] were identified from high-throughput screening using functional assays (O'Brien et al., 2003, 2004; Lindsley et al., 2004; Liu et al., 2008). Structurally diverse $\mathrm{mGlu}_{5}$ receptor PAM scaffolds compete for the common allosteric site within the 7TM used by MPEP (Gregory et al., 2013b, 2014); however, select PAMs [e.g., CPPHA and 4-butoxy- $N$-(2,4-difluorophenyl)benzamide] are thought to interact with distinct but as yet unknown site(s) within the 7TM (O'Brien et al., 2004; Chen et al., 2008; Hammond et al., 2010; Noetzel et al., 2013). Mechanistically, GGlu $_{5}$ receptor PAMs are largely considered to potentiate $\mathrm{mGlu}_{5}$ receptor activity in response to glutamate via efficacy modulation (Gregory et al., 2012); however, probe dependence can dictate the nature of these allosteric interactions with multiple PAMs reported to enhance $\left[{ }^{3} \mathrm{H}\right]$ quisqualate binding (Bradley et al., 2011; Koehl et al., 2019) as well as show different magnitudes of cooperativity depending on the orthosteric agonist used (Sengmany et al., 2017). In this respect the largest magnitude of potentiation observed 
TABLE 5

Pharmacology of commercially available group III mGlu allosteric ligands

For a complete list refer to guidetopharmacology.org.

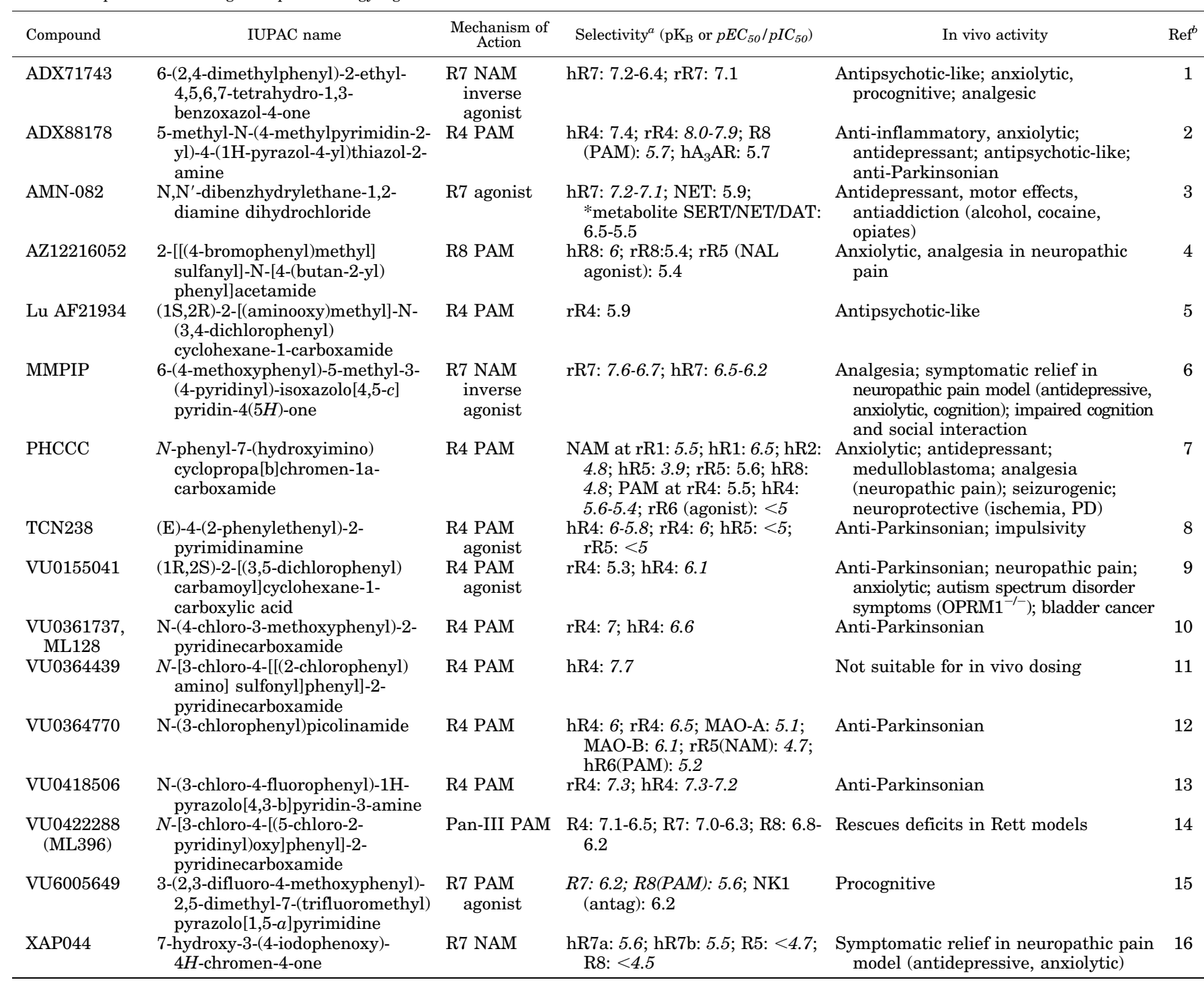

DAT, dopamine transporter; IUPAC, International Union of Pure and Applied Chemistry; MAO-A, monoamine oxydase A; MAO-B, monoamine oxidase B; NET,

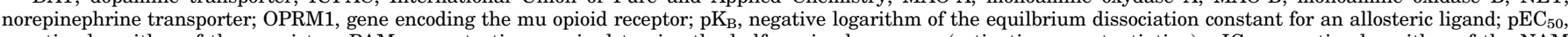

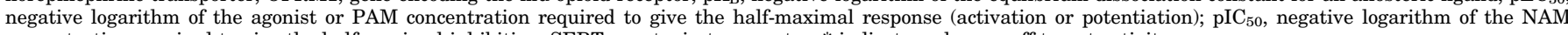
concentration required to give the half-maximal inhibition; SERT, serotonin transporter. * indicates a known off target activity.

${ }^{a}$ Where affinity estimates were unavailable $\mathrm{pIC}_{50}(\mathrm{NAMs})$ or $\mathrm{pEC}_{50}(\mathrm{PAMs})$ values are reported indicated by italics.

${ }^{b}$ 1) Kalinichev et al., 2013; Moloney et al., 2015; Cieślik et al., 2018. 2) Le Poul et al., 2012; Yin et al., 2013; Kalinichev et al., 2014; Ponnazhagan et al., 2016; Volpi et al.,

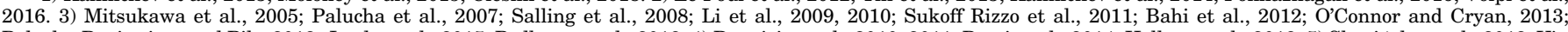

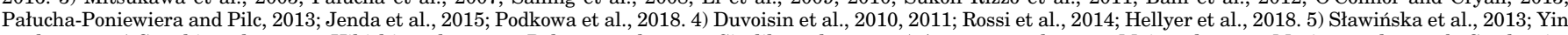

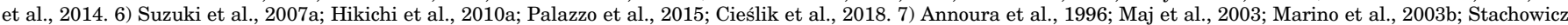

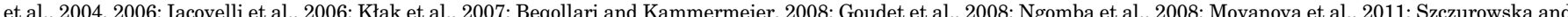

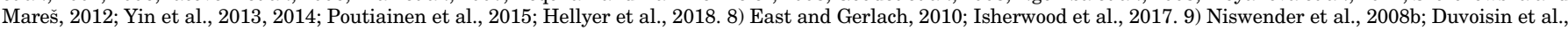

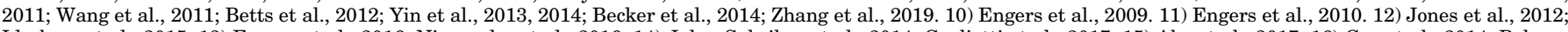

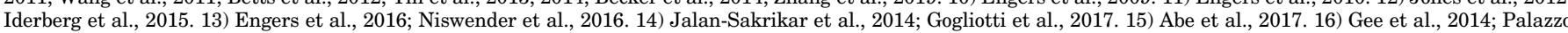
et al., 2015.

in $\mathrm{mGlu}_{5}$ receptor functional assays is $\sim 20$-fold shift in glutamate potency for 1-(4-(2,4-difluorophenyl) piperazin-1-yl)-2-((4-fluorobenzyl)oxy)ethan-1-one (DPFE) and 5-fluoro-2-\{3-[(3S,6R)-1-[(4-fluorophenyl)carbonyl]6-methylpiperidin-3-yl]-1,2,4-oxadiazol-5-yl\}pyridine (Gregory et al., 2013a; Parmentier-Batteur et al., 2014). Indeed, the magnitude of cooperativity was the best predictor of in vivo efficacy of an $\mathrm{mGlu}_{5}$ receptor PAM series using the amphetamine-induced hyperlocomotion assay, when total and free brain and plasma concentrations were determined from the same rats (Gregory et al., 2019). Select $\mathrm{mGlu}_{5}$ receptor PAMs have intrinsic agonist efficacy; however, in some cases this is linked to high receptor reserve in recombinant systems (Noetzel et al., 2012). However, this is not always the case, with some compounds, for example, DPFE, showing intrinsic efficacy in low expression and native cell systems (Gregory et al., 2013a; Sengmany et al., 2017), and may 
A<smiles>C/C=C\C(=O)OC(=O)[C@]12C[C@@H]1/C(=N\O)c1ccccc1OC2(C(=O)OCC)C(=O)OCC</smiles><smiles>[R6][R6]#[R6]c1ccc(F)cc1C1CCCN1S(=O)(=O)c1ccc(C)cc1</smiles>

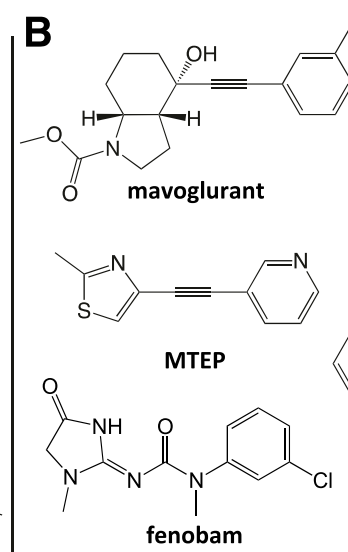

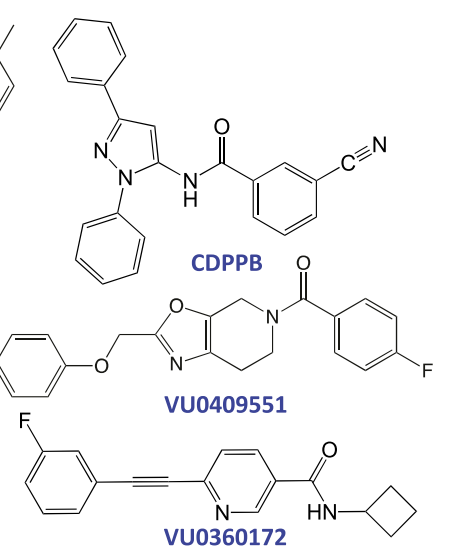

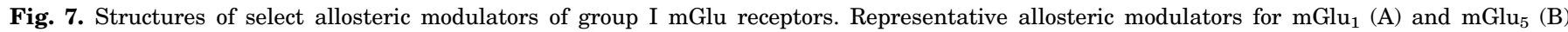

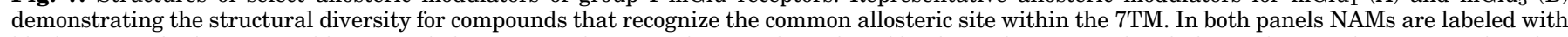

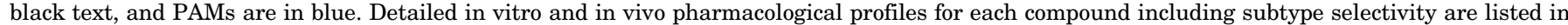
Tables 2 and 3. The following abbreviation is used: RO 01-6128, ethyl N-[2,2-di(phenyl)acetyl]carbamate.

also be dependent on measure of receptor activation, where

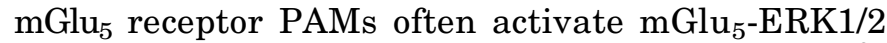
phosphorylation at concentrations that do not elicit $\mathrm{iCa}^{2+}$ mobilization responses (Gregory et al., 2012; Rook et al., 2013). Despite the complexity in pharmacology, successful discovery efforts for multiple centrally active $\mathrm{mGlu}_{5}$ receptor PAMs have established proof-of-concept for procognitive and antipsychotic efficacy of $\mathrm{mGlu}_{5}$ potentiators (Table 3). However, on-target adverse effect liability has been associated with multiple scaffolds, which has been attributed in part to intrinsic agonist activity and/or magnitude of cooperativity (Rook et al., 2013; ParmentierBatteur et al., 2014). Recent studies have challenged these conclusions (Rook et al., 2015b; Sengmany et al., 2017), suggesting that biased pharmacology of $\mathrm{mGlu}_{5}$ receptor PAMs may be linked to adverse versus therapeutic effects (discussed in detail below).

\section{E. Group II PAMs and NAMs}

Discovery and validation of group II mGlu receptor allosteric ligands has benefited from the availability of radiolabeled orthosteric agonists and antagonists owing to the generally higher affinity of orthosteric ligands for group II mGlu receptors (Table 1). Indeed, multiple different $\mathrm{mGlu}_{2}$ receptor-selective PAMs have been disclosed and established proof-of-concept for $\mathrm{mGlu}_{2}$ receptor potentiation as a viable therapeutic intervention for anxiety, psychosis, and addiction (Table 4). The first $\mathrm{mGlu}_{2}$ receptor-selective PAM was 2,2,2-trifluoro$\mathrm{N}$-[4-(2-methoxyphenoxy)phenyl]- $N$-(3-pyridinylmethyl)ethanesulfonamide (LY487379; also referred to as 4-MPPTS) (Johnson et al., 2003), which enhances radiolabeled orthosteric agonist $\left(\left[{ }^{3} \mathrm{H}\right] \mathrm{LY} 354740\right.$ and $\left.\left[{ }^{3} \mathrm{H}\right] D C G-I V\right)$ binding (Schaffhauser et al., 2003; Lundström et al., 2016), orthosteric agonist affinity (Johnson et al., 2005), and functional activity, although LY487379 shows probe dependence as it is neutral with respect to affinity of LY379268 (a high affinity orthosteric agonist) (Johnson et al., 2005). Furthermore, LY487379 has neutral cooperativity with respect to orthosteric antagonist binding (Schaffhauser et al., 2003; Johnson et al., 2005), suggesting LY487379 preferentially interacts with the active

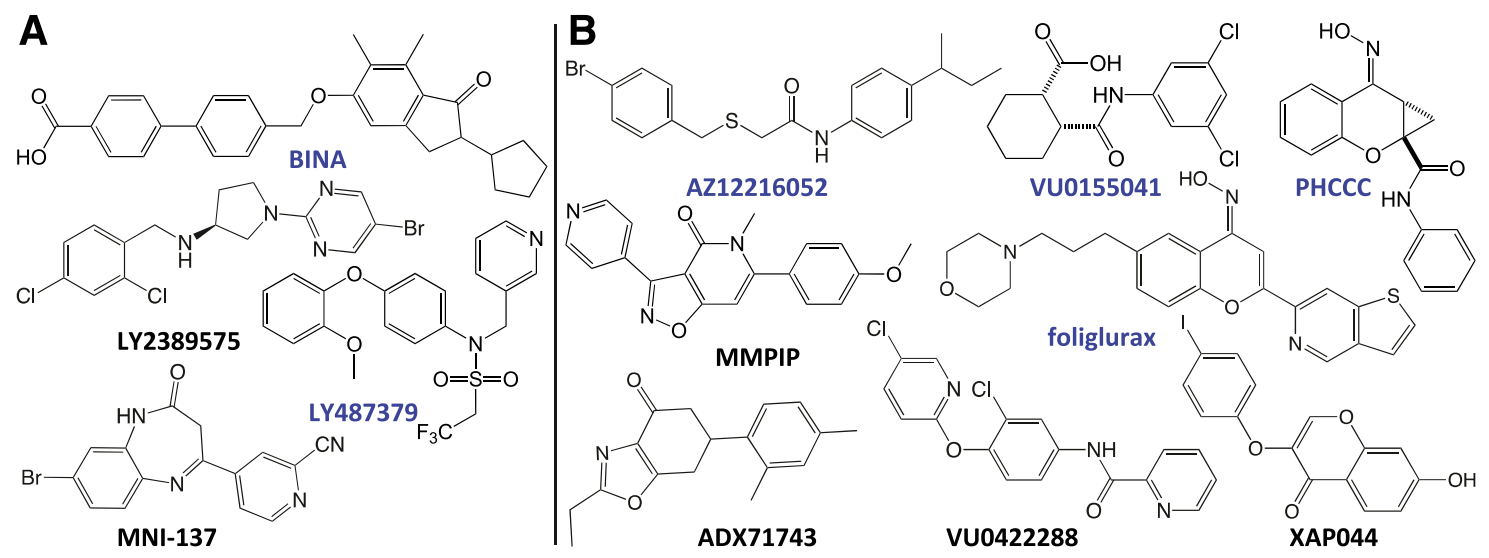

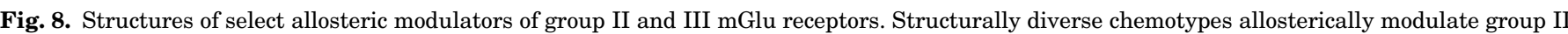

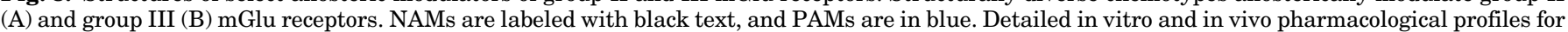

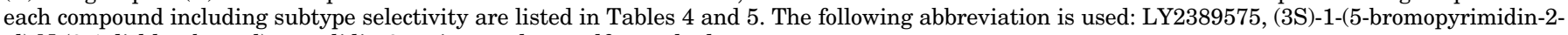
yl)-N-(2,4-dichlorobenzyl) pyrrolidin-3-amine methanesulfonate hydrate. 
receptor state. A similar pharmacological profile has been noted for $\mathrm{mGlu}_{2}$ receptor-selective PAMs from different chemotypes [including 4-[3-[(2-cyclopentyl-6,7-dimethyl1-oxo-2,3-dihydroinden-5-yl)oxymethyl]phenyl]benzoic acid (BINA), 5-(7-chloro-2-((S)-1-cyclopropyl-ethyl)-1oxo-2,3-dihydro-1H-isoindol-5-yl)-isoxazole-3-carboxylic acid dimethylamide, and 8-chloro-3-(cyclopropylmethyl)7-(4-(3,6-difluoro-2-methoxyphenyl)piperidin-1-yl)-[1,2,4] triazolo[4,3- $a$ ]pyridine], which also potentiate glutamate binding and efficacy but are neutral with respect to orthosteric antagonist binding (O'Brien et al., 2018), although the recently disclosed $\mathrm{mGlu}_{2}$ receptor modulator, (S)-2-(1,1-dimethyl-indan-5-yloxymethyl)-2,3-dihydrooxazolo[3,2-a]pyrimidin-7-one, has even more pronounced probe dependence, potentiating glutamate affinity but inhibiting $\left[{ }^{3} \mathrm{H}\right] \mathrm{LY} 341495$ binding (Griebel et al., 2016). Further insights into the mechanism of action of $\mathrm{mGlu}_{2}$ receptor PAMs have been elucidated using radiolabeled $\mathrm{mGlu}_{2}$ receptor PAMs [2,2,2-trifluoro-N-(3-pentan-2yloxyphenyl)-N-(pyridin-3-ylmethyl)ethanesulfonamide, JNJ-46281222, and 2-[[(4-bromophenyl)methyl]sulfanyl]$\mathrm{N}$-[4-(butan-2-yl)phenyl]acetamide]. Compared with orthosteric radioligands, radiolabeled $\mathrm{mGlu}_{2}$ receptor PAMs recognize fewer binding sites, which is thought to indicate occupation of a single 7TM domain within the dimeric receptor as well as preferential binding to active receptor conformations since orthosteric agonists can increase the number of $\mathrm{mGlu}_{2}$ receptor PAM binding sites (Lundström et al., 2009, 2011, 2016; Lavreysen et al., 2013; Doornbos et al., 2016; O’Brien et al., 2018). Most $\mathrm{mGlu}_{2}$ receptor PAMs have intrinsic agonist efficacy (Table 4) with the maximal degree of potentiation observed for glutamate potency of between 10- and 30-fold (Johnson et al., 2005; Galici et al., 2006; Lavreysen et al., 2015; O'Brien et al., 2018). In addition to increased selectivity over $\mathrm{mGlu}_{3}$ relative to orthosteric agents, $\mathrm{mGlu}_{2}$ receptor PAMs may also provide improved therapeutic efficacy owing to reduced capacity for induction of tolerance compared with group II receptor orthosteric agonists (Ahnaou et al., 2015). In contrast to successful $\mathrm{mGlu}_{2}$ receptor PAM discovery campaigns, to date, $\mathrm{mGlu}_{3}$ receptor-selective PAMs have remained elusive.

Discovery efforts for group II mGlu receptor NAMs have yielded both $\mathrm{mGlu}_{2}$ and $\mathrm{mGlu}_{3}$ receptor subtypeselective ligands, although there is less structural diversity available when compared with $\mathrm{mGlu}_{2}$ receptor PAMs (Fig. 8; Table 4). Negative allosteric modulators of group II mGlu receptors have demonstrated efficacy for improving cognitive deficits and reversing behaviors in preclinical models for depression and anxiety (Woltering et al., 2010; Campo et al., 2011; Goeldner et al., 2013; Engers et al., 2015, 2017) and are neuroprotective under ischemic insult (Motolese et al., 2015). Mechanistically, group II receptor NAMs [including 4-(8-bromo-5-oxo-3,4,5,6tetrahydro-1,6-benzodiazocin-2-yl)pyridine-2-carbonitrile (MNI-137), 4-[3-(2,6-dimethylpyridin-4-yl)phenyl]-7-methyl8-(trifluoromethyl)-1,3-dihydro-1,5-benzodiazepin-2-one, 5-[2-[7-trifluoromethyl)-5-[4-(trifluoromethyl)phenyl] pyrazolo[1,5-a]pyrimidin-3-yl]ethynyl]pyridin-2-amine (decoglurant), and related compounds] are neutral with respect to glutamate affinity, primarily acting as negative modulators of glutamate efficacy (Hemstapat et al., 2007; Campo et al., 2011; O'Brien et al., 2018). Akin to observations with group II receptor PAMs, select NAMs have demonstrated probe dependence with respect to modulation of orthosteric agonist affinity, where ligands related to 4-[3-(2,6-dimethylpyridin-4-yl)phenyl]-7-methyl8-(trifluoromethyl)-1,3-dihydro-1,5-benzodiazepin-2-one or decoglurant are NAMs with respect to $\left[{ }^{3} \mathrm{H}\right] \mathrm{LY} 354740$ binding (Woltering et al., 2008; Lundström et al., 2011). For the majority of pan-group II, mGlu receptor NAMs, including [2-fluoro-4-[2-(4-methoxyphenyl)ethynyl]phenyl] [(3R)-3-hydroxy-1-piperidinyl]methanone (ML337), MNI-137, and decoglurant, are full NAMs, completely abolishing the functional response to orthosteric agonists at both $\mathrm{mGlu}_{2}$ and $\mathrm{mGlu}_{3}$ receptors (Caraci et al., 2011; Wenthur et al., 2014; O'Brien et al., 2018). However, MNI-137 has differing degrees of negative cooperativity in functional assays of $\mathrm{mGlu}_{2}$ receptor activity, in some instances showing full blockade but in others limited negative cooperativity (Hemstapat et al., 2007; Yin et al., 2014; O’Brien et al., 2018). Furthermore, both the reported selectivity and inhibitory activity of $\mathrm{mGlu}_{2 / 3}$ receptor NAM (3S)-1-(5-bromopyrimidin-2-yl)-N-(2,4-dichlorobenzyl) pyrrolidin-3-amine methanesulfonate hydrate differs depending on the response measured (Caraci et al., 2011; Sheffler et al., 2012). In this respect, it is worth noting that for many ligands and series there has been limited pharmacological profiling to fully discern mechanism of action. Subtype-selective $\mathrm{mGlu}_{2}$ receptor [e.g., 6-(((2S,6R)-2,6-dimethylmorpholino)methyl)-1-(4-fluorophenyl)-4-oxo-1,4-dihydroquinoline-3-carboxamide, 4-(2-fluoro-4-methoxyphenyl)-7-(2(2-methylpyrimidin-5-yl)ethyl)quinoline-2-carboxamide, 4-(8-bromo-5-oxo-3,4,5,6-tetrahydro-1,6-benzodiazocin-2-yl) pyridine-2-carbonitrile] or $\mathrm{mGlu}_{3}$ receptor $[[(3 R)-3-$ (hydroxymethyl)-1-pipridinyl] [4-[2-(4-methoxyphenyl) ethynyl]phenyl]methanone, (S)-1-(4-fluorophenyl)-4-(2phenoxypropoxy)pyridin-2 $(1 \mathrm{H})$-one] NAMs have been reported (Kolczewski et al., 1999; Sheffler et al., 2012; Felts et al., 2015; Walker et al., 2015; Engers et al., 2017). However, the group II receptor selectivity of ML337 was recently demonstrated to be in part attributable to cooperativity, as this ligand is an NAL at $\mathrm{mGlu}_{5}$ (Hellyer et al., 2018). Whether other reportedly subtype-selective NAMs are also due to cooperativity rather than affinity remains to be elucidated.

Both selective and pan-group II receptor NAMs from diverse scaffolds interact with a common or overlapping site with that used by $\mathrm{mGlu}_{2}$ receptor PAMs within the 7TM domain (Schaffhauser et al., 2003; Rowe et al., 2008; Lundström et al., 2011, 2016; O'Brien et al., 2018). Select amino acid residues within this common site can differentially influence group II receptor NAM versus 
PAM activity (Hemstapat et al., 2007; Lundström et al., 2011; Perez-Benito et al., 2017). These differential effects may be attributable to differential effects on cooperativity or affinity whereby distinct ligand-receptor interactions may contribute to active versus inactive receptor conformations. However, for some scaffolds [e.g., 8-chloro3-(cyclopropylmethyl)-7-(4-(3,6-difluoro-2-methoxyphenyl) piperidin-1-yl)-[1,2,4]triazolo[4,3- $a]$ pyridine, decoglurant, 6-(((2S,6R)-2,6-dimethylmorpholino)methyl)-1-(4-fluorophenyl)-4-oxo-1,4-dihydroquinoline-3-carboxamide, 4-(2-fluoro-4-methoxyphenyl)-7-(2-(2-methylpyrimidin-5-yl) ethyl)quinoline-2-carboxamide], allosteric interactions have been observed with a mGlu ${ }_{2}$ receptor PAM radioligand, indicative of multiple allosteric sites within the 7TM (O'Brien et al., 2018) or possibly more complex interactions due to the dimeric nature of mGlu receptors as has been noted for other class C GPCR allosteric modulators (Gregory et al., 2018).

\section{F. Group III PAMs and NAMs}

A list of the some of the commercially available allosteric modulators of group III mGlu receptors is provided in Table 5. The first identified group III mGlu receptor-selective allosteric modulator was $N$-phenyl-7(hydroxyimino)cyclopropa[b]chromen-1a-carboxamide (PHCCC) (Maj et al., 2003; Marino et al., 2003b) (Fig. 8). PHCCC acts as an $\mathrm{mGlu}_{4}$ receptor PAM, increasing potency and efficacy of glutamate or L-AP4 in cell-based assays. PHCCC is closely related to the $\mathrm{mGlu}_{1}$ receptorselective NAM CPCCOEt (Annoura et al., 1996) (Fig. 7). Although it has weak potency and poor solubility, PHCCC provided a very useful tool to demonstrate the therapeutic potential of targeting $\mathrm{mGlu}_{4}$ receptors in Parkinson's disease and paving the way to drug candidates (Charvin, 2018). Indeed, PHCCC potentiated the inhibitory effect of L-AP4 on transmission at the striatopallidal synapse and reversed akinesia in rats (Marino et al., 2003a,b). PHCCC also reduces hyperalgesia in rat models of chronic pain (Goudet et al., 2008). Subsequently, a new $\mathrm{mGlu}_{4}$ receptor PAM named (1R,2S)-2-[(3,5-dichlorophenyl)carbamoyl]cyclohexane1-carboxylic acid (VU0155041) was discovered (Christov et al., 2011), which is more potent and more soluble than PHCCC. Interestingly, VU0155041 is an $\mathrm{mGlu}_{4}$ receptor allosteric agonist (PAM agonist), contrary to the pure PAM profile of PHCCC. PHCCC and VU0155041 do not compete for the same site (Niswender et al., 2008a). Accordingly, two partially overlapping 7TM binding pockets have been identified in $\mathrm{mGlu}_{4}$ receptors, a shallow and a deep pocket (Rovira et al., 2015). Analysis of the pharmacological properties and binding modes of several $\mathrm{mGlu}_{4}$ receptor PAMs, revealed the intrinsic efficacy and cooperativity of $\mathrm{mGlu}_{4}$ PAMs (both affinity and efficacy modulation of L-AP4 and glutamate) correlate with the binding mode (Rovira et al., 2015). PAMs with intrinsic allosteric agonism bind in the shallow pocket, analogous to the pocket of natural agonists of class A GPCRs, whereas PAMs exhibiting the highest cooperativity with orthosteric agonists bind into a deeper pocket, corresponding to that of mavoglurant in the $\mathrm{mGlu}_{5}$ receptor 7TM crystal structure (Doré et al., 2014) and pointing toward a site topographically homologous to the $\mathrm{Na}^{+}$binding pocket of class A GPCRs. In preclinical studies, VU0155041 improves symptoms of Parkinson's disease (Christov et al., 2011), chronic pain (Wang et al., 2011), and autistic-like syndromes (Becker et al., 2014). Foliglurax (PXT002331) is a derivative of $\mathrm{PHCCC}$ with good water solubility and high brain exposure after oral administration (Charvin et al., 2017). It is a potent and selective $\mathrm{mGlu}_{4}$ receptor PAM displaying strong anti-Parkinsonian activity in rodent preclinical models of Parkinson's disease (Charvin et al., 2017) as well as in primates (Charvin et al., 2018a); however, it recently failed to show efficacy in a phase II clinical trial. Several $\mathrm{mGlu}_{4}$ receptor PAMs also exhibit PAM activity on $\mathrm{mGlu}_{6}$ receptors; as yet no selective $\mathrm{mGlu}_{6}$ receptor allosteric modulators have been described.

The first allosteric modulator acting at the $\mathrm{mGlu}_{7}$ receptor to be described was $\mathrm{N}, \mathrm{N}^{\prime}$-dibenzhydrylethane1,2-diamine dihydrochloride (AMN082) (Mitsukawa et al., 2005). Since there was a lack of pharmacological tools to study $\mathrm{mGlu}_{7}$ receptor, this $\mathrm{mGlu}_{7}$ PAM agonist attracted much interest. However, AMN082 presents off-target effects, as it retains activity in $\mathrm{mGlu}_{7}$ receptor knockout mice (Ahnaou et al., 2016b). Indeed, AMN082 is rapidly metabolized in vivo, with the major metabolite being a potent monoamine transporter inhibitor (Sukoff Rizzo et al., 2011). Thus, preclinical results obtained with AMN082 have to be carefully interpreted, since its actions may not be driven solely by $\mathrm{mGlu}_{7}$ receptors. Selective $\mathrm{mGlu}_{7}$ receptor NAMs have been described and may be more adequate for investigating the role of $\mathrm{mGlu}_{7}$ receptor in vivo: 6-(4-methoxyphenyl)-5methyl-3-(4-pyridinyl)-isoxazolo[4,5-c]pyridin-4(5H)-one (MMPIP) (Suzuki et al., 2007b), 6-(2,4-dimethylphenyl)2-ethyl-4,5,6,7-tetrahydro-1,3-benzoxazol-4-one (ADX71743) (Kalinichev et al., 2013), and 7-hydroxy-3-(4-iodophenoxy)4H-chromen-4-one (XAP044) (Gee et al., 2014). Interestingly, the inhibitory activity of MMPIP is context dependent, where MMPIP may not antagonize $\mathrm{mGlu}_{7}$ receptor activity in all cellular contexts. Indeed, MMPIP is unable to block agonist-mediated responses at the Schaffer collateral-CA1 synapse, where $\mathrm{mGlu}_{7}$ receptor is known to modulate neurotransmission (Niswender et al., 2010). ADX71743 is a bioavailable and brain penetrant $\mathrm{mGlu}_{7} \mathrm{NAM}$ that induces a robust anxiolytic effect in rodents (Kalinichev et al., 2013). Most small allosteric modulators described so far act via a binding pocket located within the transmembrane domain; however, XAP044 mediates its action through an interaction with the extracellular domain of $\mathrm{mGlu}_{7}$ receptor (Gee et al., 2014). The exact binding pocket of XAP044 is not known at the moment, with chimeric $\mathrm{mGlu}_{7} / \mathrm{mGlu}_{6}$ receptors used to map its action to the 
extracellular domains (Gee et al., 2014). Recent efforts aiming to identify novel $\mathrm{mGlu}_{7}$ receptor PAM scaffolds have turned to cheminformatics-based approaches; however, to date these have yielded low potency potentiators (Tresadern et al., 2017).

Only few $\mathrm{mGlu}_{8}$ receptor allosteric modulators have been identified. 2-[[(4-Bromophenyl)methyl]sulfanyl]$\mathrm{N}$-[4-(butan-2-yl)phenyl]acetamide (AZ12216052) is an $\mathrm{mGlu}_{8}$ receptor PAM agonist of glutamate at $\mathrm{mGlu}_{8}$ receptors (Duvoisin et al., 2010). In vivo, AZ12216052 is anxiolytic in apolipoprotein E-deficient mice, which show increased levels of anxiety-like behaviors (Duvoisin et al., 2010). AZ12216052 also displays analgesic activity after injection into the dorsal striatum of neuropathic rats (Rossi et al., 2014). However, AZ12216052 possesses some off-target effects since it retains anxiolytic activity in $\mathrm{mGlu}_{8}^{-1-}$ mice (Duvoisin et al., 2011). Another useful pharmacological tool for $\mathrm{mGlu}_{8}$ receptor is VU6005649, a brain penetrant PAM of $\mathrm{mGlu}_{7}$ and $\mathrm{mGlu}_{8}$ receptors that displays in vivo efficacy in a mouse contextual fear conditioning model (Abe et al., 2017). The pan-group III receptor PAM, N-[3-chloro-4-[(5chloro-2-pyridinyl)oxy]phenyl]-2-pyridinecarboxamide (VU0422288), which has similar affinity for $\mathrm{mGlu}_{4}$, $\mathrm{mGlu}_{7}$, and $\mathrm{mGlu}_{8}$ receptors (Jalan-Sakrikar et al., 2014), rescues deficits (synaptic plasticity and behavioral phenotypes) in a mouse model of Rett syndrome (Gogliotti et al., 2017). VU0422288 also shows probe dependence with respect to both its apparent affinity and magnitude of positive cooperativity (Jalan-Sakrikar et al., 2014). Whether targeting one or multiple of the group III receptor subtypes will best treat this neurologic disorder remains to be explored with subtypeselective pharmacological agents.

\section{G. Allosteric Modulators Progressing to Clinical Trials}

With respect to clinical translation of promising preclinical efficacy for mGlu receptor allosteric modulators, $\mathrm{mGlu}_{5}$ NAMs have demonstrated the most progress with multiple agents reaching phase II trials for a variety of indications. Prior to elucidation of its mechanism of action, fenobam was assessed in a small double-blind placebo-controlled study as a single agent for treatment of anxiety compared with diazepam and was reported to have fewer adverse effects (Pecknold et al., 1982). Subsequently, fenobam was trialed in an open-label pilot study for treatment of fragile $\mathrm{X}$ syndrome behavioral deficits, where it was well tolerated but lacked efficacy (Berry-Kravis et al., 2009). Multiple phase II trials of $\mathrm{mGlu}_{5}$ receptor NAMs in patients with fragile $\mathrm{X}$ syndrome (both adolescents and adults) have now been completed, with basimglurant (RO4917523) and mavoglurant (AFQ056) being well tolerated, improving behavioral symptoms but failing to meet primary outcomes (Jacquemont et al., 2011; Bailey et al., 2016; Berry-Kravis et al., 2016; Youssef et al., 2018). These failures may in part be attributable to the difficulties associated with study design for indications lacking rigorous criteria for assessment of behavioral symptoms, or a need to stratify patient populations. Basimglurant also failed to show efficacy in primary clinician assessed measures for major depressive disorder, although patient-reported outcomes suggested an antidepressive effect (Quiroz et al., 2016). Mavoglurant entered phase II trials for obsessive-compulsive disorder but was terminated early due to lack of efficacy and a higher incidence of adverse effects (Rutrick et al., 2017). Beyond psychiatric indications, mavoglurant lacked efficacy in treating patients with levodopa-induced dyskinesia (LID) in PD or chorea in patients with Huntington's disease (Reilmann et al., 2015; Trenkwalder et al., 2016). In contrast, dipraglurant (ADX48621) was also assessed in a phase IIa trial for PD-LID, showing promising indications of antidyskinetic efficacy (Tison et al., 2016). Raseglurant (ADX10059) showed antireflux efficacy for gastroesophageal reflux disease (Keywood et al., 2009; Zerbib et al., 2010, 2011), but further development was ultimately discontinued due to liver toxicity concerns.

With respect to modulators of other mGlu receptor subtypes, building on preclinical efficacy in addiction models for nicotine and methamphetamine (Caprioli et al., 2015; Justinova et al., 2015; Li et al., 2016), AZD8529, an $\mathrm{mGlu}_{2}$ receptor PAM, is currently in phase II trials for smoking cessation but failed to demonstrate efficacy as an antipsychotic or for negative symptoms in patients with schizophrenia (Litman et al., 2016). Another $\mathrm{mGlu}_{2}$ receptor PAM, ADX71149/JNJ-40411813, was also trialed for smoking cessation and found to improve attention and memory as well as reverse effects of ketamine (Salih et al., 2015). In a phase II trial of major depressive disorder patients with significant anxiety, JNJ-40411813 as an adjunct therapy to standard of care failed to relieve anxiety (Kent et al., 2016). A group II receptor NAM, decoglurant, also commenced trials for major depressive disorder; results are yet to be posted, but development was discontinued. The efficacy

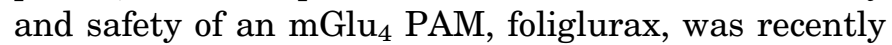
evaluated in a phase II clinical trials in patients with PD treated with levodopa, experiencing end-of-dose wearing off and levodopa-induced dyskinesia (Charvin et al., 2017, 2018a), but the program was discontinued due to insufficient efficacy. The varying degrees of success and failures with clinical translation for $\mathrm{mGlu}$ receptor allosteric modulators are in keeping with high attrition rates for neuroscience targets. Moreover, these results speak to a need to better understand the pharmacological properties of allosteric modulators and harness novel modes of action and activity.

\section{H. Secondary Allosteric Sites within the VFT and 7TM Domains}

The vast majority of allosteric modulators for mGlu receptors are believed to interact with a common allosteric site within the 7TM domain, analogous to the 
biogenic amine orthosteric binding pocket of class A GPCRs. However, allosteric modulators interacting at alternate allosteric sites may offer the means to engender unique pharmacological profiles and increased subtype selectivity. Within the context of a full-length dimeric mGlu receptor, there are multiple possible sites to exploit for allosteric ligands. Multiple subtypeselective single domain antibodies, also referred to as nanobodies, have now been described that recognize epitopes within the VFT. For $\mathrm{mGlu}_{2}$, three nanobodies that recognize overlapping epitopes but have different pharmacological properties have been described (Scholler et al., 2017). DN1 recognizes both active and inactive $\mathrm{mGlu}_{2}$ receptors, whereas $\mathrm{DN} 10$ and $\mathrm{DN} 13$ require active homodimeric $\mathrm{mGlu}_{2}$ receptor states to bind and potentiate orthosteric agonist activity (Scholler et al., 2017). Similarly, a nanobody that recognizes a loop region with lobe 1 of the $\mathrm{mGlu}_{5}$ receptor VFT potentiates agonist binding and function but can also recognize both active and inactive receptor states (Koehl et al., 2019). To date, nanobodies have been used to facilitate structural studies or as biosensors for active receptor conformations. For $\mathrm{mGlu}_{7}$ receptors, a monoclonal antibody, MB1/28, binds to the dimeric VFT, inhibiting receptor activation but is able to induce receptor internalization (Ullmer et al., 2012). Beyond antibodies, the naturally sourced sweet protein monellin was recently revealed as an $\mathrm{mGlu}_{5}$ receptor allosteric agonist that is also thought to interact with the $\mathrm{N}$ terminus, and interacts allosterically with small molecule allosteric modulators (Chen et al., 2020).

Multiple allosteric sites have been postulated for the $\mathrm{mGlu}_{5}$ receptor 7TM domain; however, the precise location of these secondary allosteric sites has proven elusive (Chen et al., 2008; Hammond et al., 2010; Noetzel et al., 2013). With the recent publication of a full-length cryo-electron microscopy structure of $\mathrm{mGlu}_{5}$ receptors, we now appreciate that the cysteinerich domain is a stalk that holds the VFT above the 7TM domains and interacts with the second extracellular loop to transmit conformational changes (Koehl et al., 2019). It is tempting to speculate that the inability to identify these secondary pockets may have been due to a monomeric view of the 7TM domain. However, biophysical studies as well as the new structures demonstrate that the 7TM domains themselves dimerize when activated (El Moustaine et al., 2012; Doumazane et al., 2013; Xue et al., 2015; Koehl et al., 2019). Appreciation of the full-length dimeric structure offers the possibility of identifying new allosteric sites to exploit through targeting these newly appreciated interfaces.

\section{Evolving Concepts}

\section{A. Biased Modulators}

Allosteric modulators elicit potentiation or inhibition through stabilizing different receptor conformations than can be achieved with an orthosteric ligand alone.
Therefore, there is the potential that these conformations can give rise to biased pharmacology. Where intrinsic efficacy differs between pathways relative to a reference agonist, this is referred to as biased agonism and can be quantified as discussed previously for biased orthosteric agonists. For mGlu ${ }_{5}$ receptors, PAM agonists from diverse scaffolds are biased agonists relative to DHPG in both recombinant and native cells; however, the bias profile differs between scaffolds, with 5-[2-(2-(3-fluorophenyl)ethynyl]- $N$-[(1R)-2-hydroxy1,2-dimethylpropyl]-2-pyridinecarboxamide, DPFE, and [6,7-dihydro-2-(phenoxymethyl)oxazolo[5,4-c]pyridin-5(4H)yl](fluorophenyl)methanone (VU0409551) each exhibiting different bias profiles for $\mathrm{mGlu}_{5}$ receptor signaling and receptor desensitization (Sengmany et al., 2017; Hellyer et al., 2019). However, biased modulation is also possible, where the direction or magnitude of modulation of the same agonist differs between pathways and may manifest either as differential apparent affinity or cooperativity (Sengmany et al., 2019). For structurally diverse $\mathrm{mGlu}_{5}$ receptor PAMs (VU0360172, DPFE, VU0409551), the magnitude of cooperativity with DHPG was lower when measured in $\mathrm{IP}_{1}$ accumulation compared with $\mathrm{iCa}^{2+}$ mobilization (Sengmany et al., 2017). Moreover, DPFE and CDPPB inhibit mGlu receptor orthosteric agonist stimulated ERK1/2 phosphorylation in primary cultures (Zhang et al., 2005; Sengmany et al., 2017). Importantly, biased allosteric agonism and potentiation of $\mathrm{mGlu}_{5}$ receptors, as well as probe dependence, are mediated via dynamic interactions within the common allosteric pocket (Hellyer et al., 2020; Sengmany et al., 2020). Allosteric interactions with distinct binding sites would be expected to offer further diversity in these biased pharmacological fingerprints. Furthermore, differential cooperativity has also been noted for $\mathrm{mGlu}_{5}$ receptor NAMs, where $\mathrm{N}$-(3-chloro-2-fluorophenyl)-3-cyano-5-fluoro-benzamide inhibits $\mathrm{iCa}^{2+}$ mobilization but is an NAL with respect to $\mathrm{IP}_{1}$ accumulation (Sengmany et al., 2019). Recent studies indicate biased agonism and modulation of $\mathrm{mGlu}_{5}$ receptor NAMs and PAMs extends to receptor regulatory processes such as internalization and desensitization (Hellyer et al., 2019; Arsova et al., 2020). Another contributing factor to biased modulator pharmacology at $\mathrm{mGlu}_{5}$ receptors may be ligand binding kinetics as suggested by two recent studies on structurally diverse NAMs (Sengmany et al., 2019; Arsova et al., 2020).

Biased pharmacology of $\mathrm{mGlu}_{5}$ receptor PAMs extends beyond second messenger signaling in cultures to intact circuitry. VU0409551 potentiates mGlu -de- $^{-}$ pendent long-term depression in the hippocampus (Rook et al., 2015b), prefrontal cortex (Ghoshal et al., 2017), and nucleus accumbens (Turner et al., 2018) but unlike other PAMs is unable to potentiate DHPG stimulation of $\mathrm{mGlu}_{5}$ receptor-mediated modulation of NMDA receptor currents (Rook et al., 2015b). Differential potentiation of $\mathrm{mGlu}_{5}$ receptor-dependent synaptic 
plasticity and modulation of NMDA receptor activity in the hippocampus has also been noted for mGlu ${ }_{5}$ receptor PAMs structurally unrelated to VU0409551. $N$ (1,3-Diphenyl-1H-pyrazolo-5-yl)-4-nitrobenzamide (VU29) potentiates hippocampal long-term potentiation but not NMDA receptor currents (Xiang et al., 2019). Within these brain slice electrophysiology experiments, mGlu $_{5}$ receptor PAM effects are mediated via enhancement of endogenous glutamate tone, or exogenously applied DHPG, suggesting that similar to VU0409551, VU29 has biased cooperativity between these two measures of $\mathrm{mGlu}_{5}$ activity. Another structurally unrelated $\mathrm{mGlu}_{5}$ receptor PAM, N-[4-chloro-2-[(4-fluoro-1,3-dioxoisoindol-2-yl)methyl]phenyl]pyridine-2-carboxamide, failed

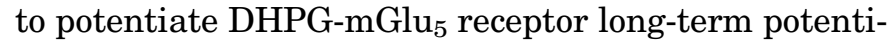
ation and long-term depression in brain slice electrophysiology experiments (Noetzel et al., 2013). For mGlu receptor allosteric ligands, biased pharmacology may offer the means to selectively modulate therapeutically beneficial effects while avoiding those linked to adverse effects. To realize this potential, there remains a need to better understand how different $\mathrm{mGlu}_{5}$ receptor signaling and cellular responses are linked to behavioral effects in the whole animal and the translation of these effects to the clinic. By example, it was recently demonstrated for a series of $\mathrm{mGlu}_{5}$ receptor PAMs closely related to VU0409551 that cooperativity with glutamate (for $\mathrm{iCa}^{2+}$ mobilization) rather than ligand affinity was predictive of relative efficacy in rats for reversing amphetamine-induced hyperlocomotion (Gregory et al., 2019). However, whether $\mathrm{mGlu}_{5}$ receptor PAM affinity, cooperativity, bias, or agonism proves to be the best predictor for therapeutic efficacy and safety across multiple in vivo measures for different scaffolds remains to be rigorously tested.

Beyond $\mathrm{mGlu}_{5}$ receptors, the $\mathrm{mGlu}_{7}$ receptor-targeting monoclonal antibody, MB1/28, is an NAM for orthosteric agonist inhibition of cAMP accumulation but has intrinsic efficacy for inducing receptor internalization (Ullmer et al., 2012). At $\mathrm{mGlu}_{2}$ receptors, the PAM agonist BINA was found to be a biased agonist relative to LY354740, favoring ERK1/2 phosphorylation over coupling to $\mathrm{G} \alpha_{15}$ (Hellyer et al., 2020). Furthermore, biased pharmacology can contribute to apparent selectivity of allosteric ligands. Reportedly, selective allosteric ligands across the class C GPCR family were recently shown to either have neutral cooperativity for mGlu receptors or have biased pharmacology (Hellyer et al., 2018). Collectively, the potential for biased allosteric ligand pharmacology highlights the importance of considering the assay/system/approach used to define mechanism of action, classify pharmacological effects, and selectivity.

\section{B. Location- and Context-Dependent Pharmacology}

Related to the phenomenon of biased allosteric agonism and modulation is the concept of context-dependent pharmacology, including the contribution of "location bias." Quite simply put, the observed pharmacological effect is influenced by the cellular context within which it is studied. The first report for context-dependent pharmacology was for the $\mathrm{mGlu}_{7}$ receptor NAM, MMPIP, which shows different magnitudes of inhibition of $\mathrm{mGlu}_{7}$ receptor activity for the same agonist between different recombinant cell lines (Niswender et al., 2010). For $\mathrm{mGlu}_{5}$ receptor modulators, both PAMs and NAMs have context-dependent pharmacology, manifested as distinct biased agonism profiles (Sengmany et al., 2017; Hellyer et al., 2019) or differential apparent affinities (Sengmany et al., 2019) or potencies (Jong et al., 2019) of NAMs between recombinant and native cells from different brain regions. Quantitative pharmacological differences between cell types may be a consequence of different stimulus-response coupling efficiencies, the presence or absence of receptor interacting proteins (other GPCRs, transducers, or scaffolding partners), or differences in receptor subcellular compartmentalization and relative accessibility by different ligands.

Group I mGlu receptors associate with different lipid microdomains (Burgueño et al., 2003; Francesconi et al., 2009b), the balance of which can be altered by receptor activation or membrane cholesterol content, which in turn can modulate signaling to ERK1/2 phosphorylation (Kumar et al., 2008, 2013). Furthermore, mGlu $_{5}$ is also found on intracellular membranes (e.g., nucleus; Jong et al., 2005), with signaling arising from these intracellular sites differing from that elicited by plasma membrane receptors (Jong et al., 2009; Kumar et al., 2012; Purgert et al., 2014). Subcellular compartmentalization of mGlu receptors can shape the physiologic responses to orthosteric agonists, particularly for surrogate agonists that cannot access intracellular receptors. Such effects may contribute to observations of probe dependence by allosteric ligands. To date, the influence of allosteric ligands on GPCR subcellular localization (and vice versa) has been relatively unexplored. However, Ca-sensing receptor allosteric ligands can act as "pharmacochaperones" to increase Ca-sensing receptor cell surface expression (White et al., 2009), suggesting that mGlu receptor allosteric ligands may also have the potential to alter receptor location.

In addition to physiologic context differences, the disease state can also impact mGlu receptor signaling and subsequently ligand pharmacology. In the setting of melanoma, $\mathrm{mGlu}_{1}$ loses the capacity to signal via classic $G$ protein pathways; however, glutamate retains the ability to stimulate $\mathrm{mGlu}_{1}$ internalization, which promotes melanoma cell survival (Gelb et al., 2015b). In a preclinical model of Huntington's disease, the balance of $\mathrm{mGlu}_{5}$ receptor signaling pathways are perturbed, where $\mathrm{IP}_{1}$ accumulation is reduced, but $\mathrm{iCa}^{2+}$ mobilization, Akt, and ERK1/2 phosphorylation are increased (Ribeiro et al., 2010). Brain region-specific changes in group I mGlu receptor signaling have also been noted 
after chronic cocaine administration, such that the $\mathrm{mGlu}_{5}$ receptor NAM MPEP becomes an agonist for inducing cAMP response element-binding protein phosphorylation in the nucleus accumbens but not striatum (Hoffmann et al., 2017). Changes in the balance of intracellular signaling responses specific to the disease setting could be exploited by the development of biased ligands; however, they also offer another layer of complexity with respect to designing appropriate discovery pipelines for the translation of biased ligands. Indeed, VU0409551, which does not potentiate mGlu receptor modulation of NMDA receptor currents in wild-type animals, does potentiate these responses in a genetic model of schizophrenia (Balu et al., 2016). These data highlight the paucity in our understanding of how different diseases and pathologic processes reshape the intracellular responses to mGlu receptors. Parsing out these differences will offer the prospect for rational discovery efforts to tailor therapeutic efficacy to the pathway level to restore neurotransmission to the healthy setting.

\section{Heteromerization of mGlu Receptors}

Historically, mGlu receptors were believed to form strict homodimers, unlike other class C GPCRs such as the obligatory heterodimeric $\mathrm{GABA}_{\mathrm{B}}$ or sweet or umami taste receptors. The first evidence of $\mathrm{mGlu}$ receptor heteromers came from the demonstration that group I receptors can assemble and function together when cotransfected in HEK293 cells but cannot assemble with either group II or group III receptors (Doumazane et al., 2011). This same study demonstrated that heteromers are also formed among group II and group III receptors, prompting investigations into the existence and function of native mGlu receptors heteromers in vivo.

One anticipates that the formation of heterocomplexes by mGlu receptors, where natively expressed in the same cells, should result in specific pharmacological signatures that differ from homomeric receptors. Intriguing pharmacological responses aroused suspicion on the existence of $\mathrm{mGlu}_{2}-\mathrm{mGlu}_{4}$ receptor heteromers in rat dorsal striatum where the well established $\mathrm{mGlu}_{4}$ receptor PAM PHCCC failed to potentiate $\mathrm{mGlu}_{4}$ receptor activity at corticostriatal synapses in rat dorsal striatum, whereas VU0155041 retained its expected $\mathrm{mGlu}_{4}$ receptor PAM activity (Yin et al., 2014). In vitro studies confirmed that $\mathrm{mGlu}_{2}-\mathrm{mGlu}_{4}$ receptor heteromers are differentially potentiated by $\mathrm{mGlu}_{4}$ receptor PAMs from different scaffolds (Kammermeier, 2012; Yin et al., 2014; Niswender et al., 2016). At the mechanistic level, these pharmacological differences arise from complex asymmetric functioning of $\mathrm{mGlu}_{2}$ $\mathrm{mGlu}_{4}$ receptor heteromers. Indeed, after orthosteric agonist activation, the signaling of $\mathrm{mGlu}_{2}-\mathrm{mGlu}_{4}$ receptor heteromer only occurs through the transmembrane domain of $\mathrm{mGlu}_{4}$ receptor (Liu et al., 2017); however, the $\mathrm{mGlu}_{2}$ receptor subunit can signal if potentiated by an $\mathrm{mGlu}_{2}$ receptor PAM. This is reminiscent of previous studies showing that only one subunit is active at a time in an mGlu receptor homodimer (Goudet et al., 2005; Hlavackova et al., 2005). Evidence of the presence of $\mathrm{mGlu}_{2}-\mathrm{mGlu}_{4}$ receptor heteromers at this corticostriatal synapses were reinforced by immunoprecipitation studies showing the presence of protein complexes containing $\mathrm{mGlu}_{2}$ and $\mathrm{mGlu}_{4}$ receptors in striatum (Yin et al., 2014). Pharmacological evidence of $\mathrm{mGlu}_{2}-\mathrm{mGlu}_{4}$ receptor heteromers has also been detected in lateral perforant path terminals in rat hippocampus (Moreno Delgado et al., 2017).

Recently, $\mathrm{mGlu}_{2}-\mathrm{mGlu}_{7}$ receptor heteromers were reported in the hippocampus and $\mathrm{mGlu}_{3}-\mathrm{mGlu}_{7}$ receptor heteromers in the cortex (Habrian et al., 2019). Interestingly, further in vitro investigations using a single molecular Förster resonance energy transfer approach revealed that the glutamate affinity and efficacy at $\mathrm{mGlu}_{7}$ receptors are greatly enhanced when associated to an $\mathrm{mGlu}_{2}$ receptor subunit, as compared with the $\mathrm{mGlu}_{7}$ receptor homodimer. Also, association with $\mathrm{mGlu}_{2}$ receptors confers to the $\mathrm{mGlu}_{7}$ receptor subunit the ability to be fully activated by the selective group III agonist LSP4-2022. Of note, previous neuroanatomical study revealed that $\mathrm{mGlu}_{7}$ and $\mathrm{mGlu}_{8}$ receptors may be expressed in the same boutons in the hippocampus (Ferraguti et al., 2005), raising the possibility the $\mathrm{mGlu}_{7}-\mathrm{mGlu}_{8}$ receptor heteromers may also be of relevance in the hippocampus. Heterodimerization of $\mathrm{mGlu}_{7}$ receptors with other mGlu receptor subtypes may provide a means to enhance the range of glutamate concentrations sensed by the $\mathrm{mGlu}_{7}$ receptor, which is otherwise insensitive to low glutamate levels.

There is also evidence of $\mathrm{mGlu}_{1}$ and $\mathrm{mGlu}_{5}$ receptors forming complexes in mouse hippocampus and cortex as shown by a knockout-controlled interaction proteomics strategy and further confirmed by immunoprecipitation and superresolution microscopy imaging of hippocampal primary neurons revealing $\mathrm{mGlu}_{1}-\mathrm{mGlu}_{5}$ receptor coexpression at the synaptic level (Pandya et al., 2016). Indeed, these data are in keeping with evidence that blockade of both group I receptor subtypes is required to ablate DHPG-induced long-term depression in the hippocampus (Volk et al., 2006). More recently, a single-cell RNA sequencing study revealed the coexpression of different mGlu subtypes within the same cell in the adult mouse cortex (Lee et al., 2020). Notably, most pyramidal cells contained at least four receptor subtypes. Probing the propensity of different mGlu receptors to coassemble by fluorescent-based complementation assays, the authors concluded that $\mathrm{mGlu}_{2}$ and $\mathrm{mGlu}_{3}$ receptors are particularly prone to form heteromers when coexpressed in heterologous cells (Lee et al., 2020). Other prominent mGlu receptor pairs included $\mathrm{mGlu}_{2 / 4}, \mathrm{mGlu}_{1 / 5}, \mathrm{mGlu}_{3 / 4}$, and $\mathrm{mGlu}_{3 / 7}$. The coexpression of native $\mathrm{mGlu}_{2}$ and $\mathrm{mGlu}_{3}$ receptors in mouse frontal cortex was confirmed by in situ 
hybridization and coimmunoprecipitation (Lee et al., 2020). The prevalence of heteromerization between mGlu receptors adds considerable complexity to understanding and interpreting molecular pharmacological properties of ligands and particularly the notion of selectivity.

Beyond heteromerization with other mGlu receptor subtypes, increasing evidence suggests mGlu receptors form heteromers and larger-order complexes with class A GPCRs, including mGlu ${ }_{2}-5-\mathrm{HT}_{2 \mathrm{~A}}$ receptors (GonzálezMaeso et al., 2008; Fribourg et al., 2011; Delille et al., 2013; Moreno et al., 2013; Moreno et al., 2016; Felsing et al., 2018), group I receptors with multiple adenosine receptor subtypes (Ciruela et al., 2001; Ferré et al., 2002; Nishi et al., 2003; Domenici et al., 2004; Rodrigues et al., 2005), $\mathrm{mGlu}_{5}$-dopamine $\mathrm{D}_{1}$ receptors (Sebastianutto et al.,

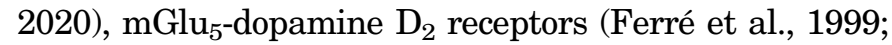
Popoli et al., 2001), and $\mathrm{mGlu}_{5}$-dopamine $\mathrm{D}_{2}$-adenosine $\mathrm{A}_{2 \mathrm{~A}}$ receptors (Díaz-Cabiale et al., 2002; Cabello et al., 2009). For each pairing with a class A GPCR, the functional responses arising when receptors are coactivated or coincidentally inhibited changes the pharmacological profile to when mGlu receptor is activated in isolation. By example, heteromerization with dopamine $\mathrm{D}_{1}$ receptors enhances the proportion of $\mathrm{mGlu}_{5}$ receptors in active states, elevating basal $\mathrm{G}_{\mathrm{q}}$ coupling and signaling toward $\mathrm{iCa}^{2+}$ mobilization over cAMP pathways (Sebastianutto et al., 2020). Heteromerization is often observed in a cell type- or brain region-specific fashion. In this respect, selectively targeting mGlu receptor heteromers offers the intriguing prospect of achieving tissue-level selectivity of drug action. The study of mGlu receptors heteromers is still in its infancy, and it is clear that further investigations will be needed to better understand its functional consequences in brain function and therapeutic potential.

\section{Optical Tools to Probe and Control mGlu Receptors}

Irreversible ligands or photoaffinity probes have been widely used to study ligand-receptor interactions and aid structural determinations across diverse protein targets. Such tools have not been available for mGlu receptors; however, recent efforts exploiting selective allosteric chemotypes have proven successful. The first in class were bifunctional $\mathrm{mGlu}_{5}$ receptor NAMs that included a photoactivatable moiety to irreversibly bind receptors and a click chemistry handle to allow secondary attachment of clickable reporter (e.g., fluorophore) for identification (Gregory et al., 2016). Installation of a covalent or photoreactive moiety has been successfully achieved within three distinct $\mathrm{mGlu}_{2}$ receptor PAM scaffolds (Doornbos et al., 2019; Hellyer et al., 2020). The development of covalent or photoactivatable ligands is not without its challenges. Covalent ligands require proximity to an appropriate amino acid for reactivity. Furthermore, the bifunctional clickable photoprobes for $\mathrm{mGlu}_{2}$ and $\mathrm{mGlu}_{5}$ receptors revealed substantial nonspecific interactions, which may limit how these tools can be applied. There has been considerable interest in alternative approaches to optically control mGlu receptor function. Two main strategies exist to control mGlu receptors by light: an optogenetic pharmacology approach based on attached photoswitchable ligands (Fig. 9) and a photopharmacology approach based on freely diffusible light-operated ligands (Fig. 10) (Goudet et al., 2018). The aim of both strategies is to use light to achieve precise spatiotemporal control over receptor activity.
A

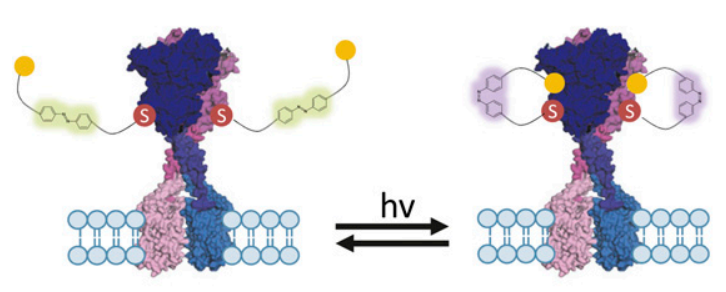

C

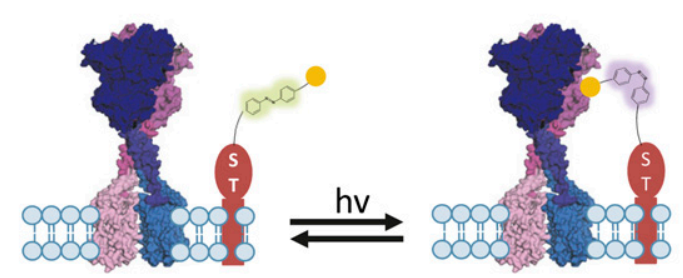

B

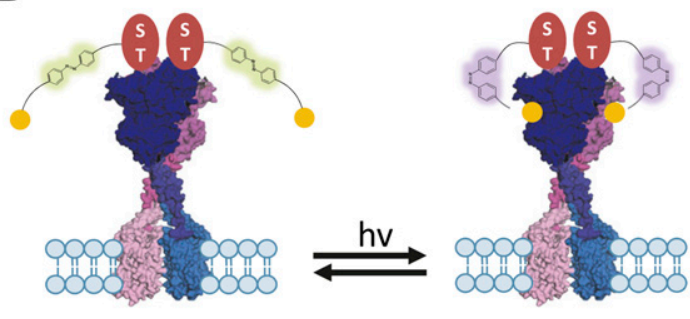

D

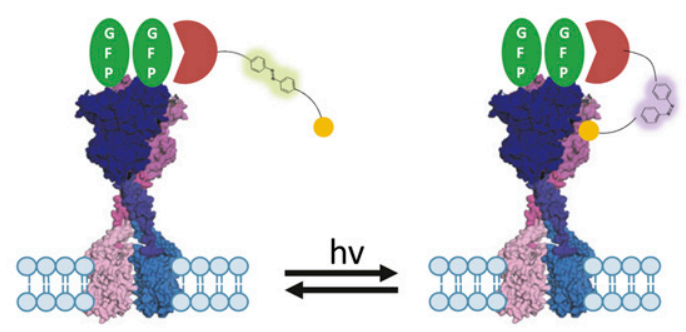

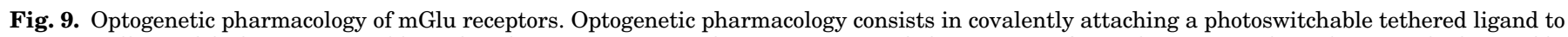

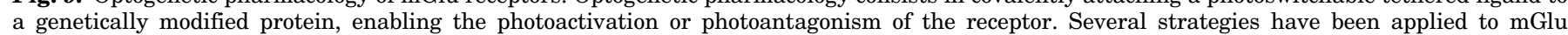

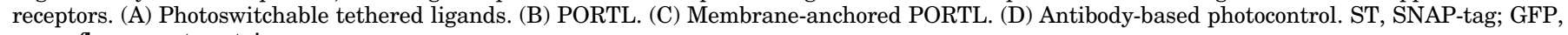
green fluorescent protein. 
A

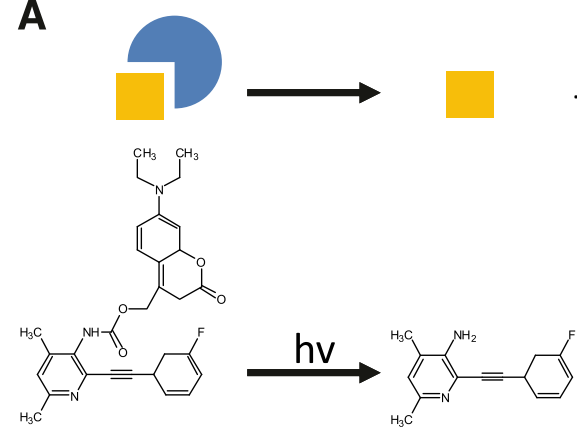

Caged-raseglurant

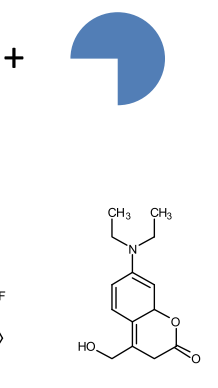

DEACM

B

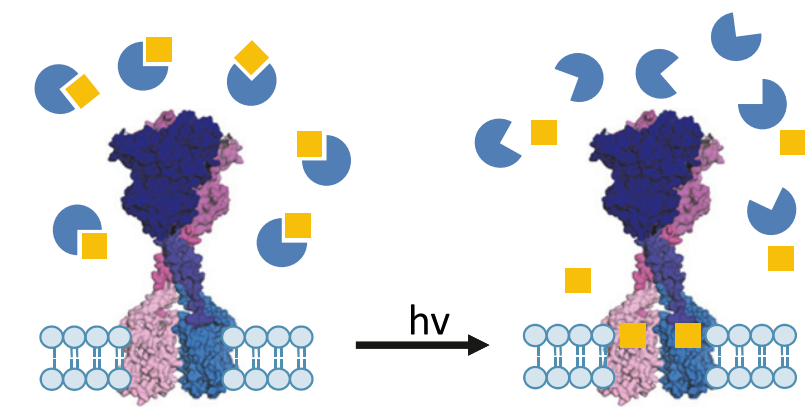

C

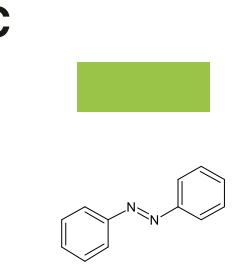

Trans-Azobenzene

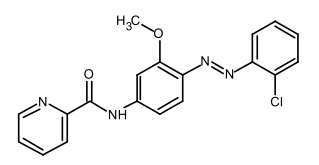

Trans-Optogluram
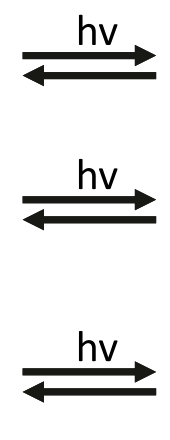

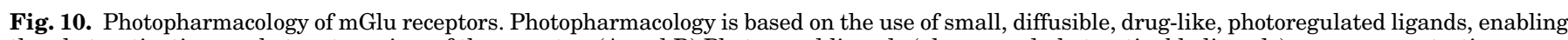

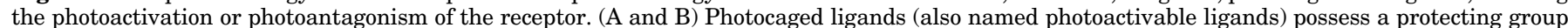

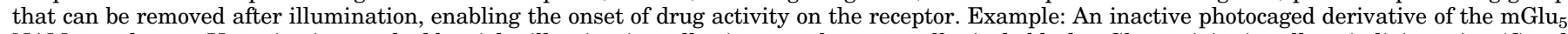

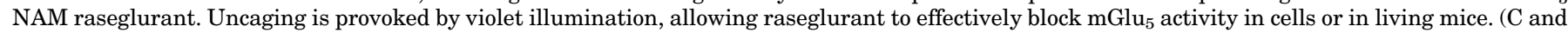

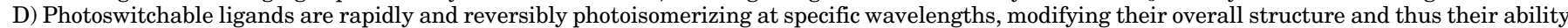

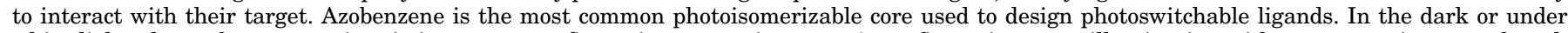

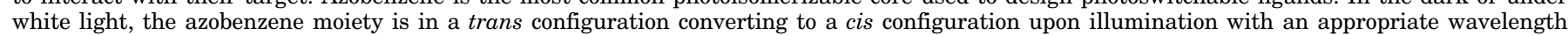
(usually in the UV range). Example: Optogluram, a photoswitchable mGlu 4 PAM. DEACM, 7-(diethylamino)coumarin-4-yl]methyl.

1. Optogenetic Pharmacology. Optogenetic pharmacology consists of covalently attaching a photoswitchable tethered ligand to a genetically modified protein (Kramer et al., 2013), which will then enable the photoactivation or photoantagonism of the receptor. In most cases, the receptor itself is modified to allow anchoring of the photoswitchable ligand, but several variants of this technique have been developed, using 1) a transmembrane protein at the proximity of the receptor or 2) an antibody targeting the receptor (Fig. 9). Optogenetic pharmacology consists of covalently attaching a photoswitchable tethered ligand to a genetically modified protein (Kramer et al., 2013), which will then enable the photoactivation or photoantagonism of the receptor.

Optogenetic pharmacological approaches allows for greater selectivity for studying the functional roles of a target receptor. The attached photoswitchable ligand can rapidly oscillate between activating and inactivating a receptor, thus being a useful optogenetic tool to understand mGlu receptor activation mechanisms (Levitz et al., 2016) or to study synaptic activity of neural circuits with high spatiotemporal resolution and pharmacological specificity. The drawback is the requirement for genetic manipulation, which can limit in vivo application, but this can be circumvented by using a viral infection approach (Acosta-Ruiz et al., 2020).

The first generation of light-controlled mGlu receptors was based on photoswitchable tethered ligands, which contain glutamate linked via a photoisomerizable azobenzene linker to a maleimide that reacts with a free cysteine within the receptor. These molecules, called MAGs, bind covalently to genetically engineered mGlu receptors that possess geometrically appropriate cysteine attachment points (Fig. 9A). Light-controlled $\mathrm{mGlu}_{2}$, $\mathrm{mGlu}_{3}$, and $\mathrm{mGlu}_{6}$ receptors were designed using this strategy. Precise optical control can be achieved in cells, in mouse brain slices, and in living zebrafish (Levitz et al., 2013). An improved spatiotemporal resolution can also be achieved via two-photon activation of lightcontrolled mGlu receptors (Carroll et al., 2015).

A second generation of light-controlled mGlu receptors has been developed based on photoswitchable orthogonal remotely tethered ligands (PORTL) and the SNAP tag technology (Keppler et al., 2003) (Fig. 9B). The photoswitchable ligands are composed of a glutamate moiety, followed by a long flexible linker 
containing an azobenzene and a benzylguanine that anchor the PORTL to a SNAP tag. The receptor is genetically modified to contain a SNAP tag at the N terminus. Interestingly, the same principle can be applied to CLIP-tagged receptors (Gautier et al., 2008). Since SNAP tag and CLIP tag possess orthogonal substrate specificities, SNAP- and CLIP-tagged proteins can be labeled simultaneously and specifically with different molecular probes in living cells. This has proven to be a very useful approach to analyze cell surface protein complexes and notably led to the discovery of specific heterodimeric mGlu receptors (Doumazane et al., 2011). By combining SNAP- and CLIP-tagged receptors and specific PORTL, Levitz and colleagues have created a family of light-gated group II/III mGlu receptors (Levitz et al., 2017), allowing multiplexed orthogonal optical control within homo or heterodimers. Optimized branched photoswitchable ligands have recently been developed permitting photoagonism of mGlu receptors with near-complete efficiency (Acosta-Ruiz et al., 2020). The PORTL strategy has been applied to $\mathrm{mGlu}_{2}$ receptor permitting light control of $\mathrm{mGlu}_{2}$ receptor-induced excitability in heterologous cells or transfected neurons (Broichhagen et al., 2015) and, more recently, to control working memory in mice (Acosta-Ruiz et al., 2020).

Alternative optogenetic pharmacology approaches have been developed. For example, tethered ligands have been designed to target a genetically modified plasma membrane protein bearing a SNAP tag, rather than directly targeting the receptor. This new approach is named membrane-anchored PORTL (Donthamsetti et al., 2019). These ligands anchor to the SNAP-tagged protein at the plasma membrane and come into close proximity to their target receptor via lateral diffusion to enable interaction (Fig. 9C). An alternative strategy consists in using ligands tethered to a SNAP-tagged antibody or nanobody targeting the receptor of interest (Fig. 9D). Proof of concept has been established using a nanobody recognizing a green fluorescent protein fused to the $\mathrm{N}$ terminus of $\mathrm{mGlu}_{2}$ receptors, allowing photocontrol of the receptor (Farrants et al., 2018).

2. Photopharmacology. Photopharmacology is based on the use of small, diffusible, drug-like, photoregulated ligands to control the function of a given target through light. Two types of freely diffusible photoregulated drugs have been developed for mGlu receptors photopharmacology: photocaged ligands and photoswitchable ligands (Fig. 10). Photopharmacological agents constitute powerful tools to manipulate and explore the function and therapeutic potential of endogenous receptors in living animals. Indeed, one of the main interests of photopharmacology resides in the ability to target endogenous receptors in native environments. Indeed, this technique does not require exogenous expression of light-controlled proteins or genetically modified receptor as with optogenetics or optogenetic pharmacology. Compared with tethered photoswitchable ligands, the pharmacological response kinetics can be slower. As with classic drugs, selectivity, pharmacokinetic, and absorption, distribution, metabolism, and excretion properties are also key considerations (Berizzi and Goudet, 2020). Another limitation is the local delivery of drug and light in vivo; however, this can be achieved using optic fibers coupled to a light source, as recently exemplified (Font et al., 2017; Zussy et al., 2018).

Photocaged ligands, also named photoactivatable ligands, possess a protecting group that can be removed after illumination, enabling the uncaged ligand to bind to its receptor (Fig. 10A). Therefore, these ligands are inactive photocaged ligands that are turned $\mathrm{ON}$ by light, enabling a precise spatiotemporal control of the onset of drug activity. Caged glutamate was developed in the 1990s and was most notably used for mapping neuronal circuits (Callaway and Katz, 1993) or for studying mGlu receptor function (Crawford et al., 1997). However, the use of caged glutamate is somehow limited due to the lack of subtype selectivity, leading to the development of ionotropic and metabotropic glutamate receptor-selective compounds. The first mGlu subtype-selective caged compound is (7-(diethylamino)2-oxo-2H-chromen-4-yl)methyl (2-((3-fluorophenyl)ethynyl)-4,6-dimethylpyridin-3-yl)carbamate, an inactive photocaged derivative of the $\mathrm{mGlu}_{5}$ receptor NAM raseglurant (Font et al., 2017) (Fig. 10B). The release of raseglurant is induced by a violet illumination, effectively blocking $\mathrm{mGlu}_{5}$ receptor activity in cells or in living mice. Interestingly, the caged compound can be injected systemically in preclinical murine models of chronic pain and uncaged locally by illumination, revealing the analgesic potential of $\mathrm{mGlu}_{5}$ blockade in peripheral tissues or in the thalamus (Font et al., 2017).

Photoswitchable ligands are designed to be rapidly and reversibly switched ON and OFF (Fig. 10C). Typical ligand design comprises incorporating a photoswitchable core into an active moiety that is selectively recognized by the target receptor. The photoswitchable core reversibly photoisomerizes at specific wavelengths, modifying the overall structure of the ligand and thus its ability to interact with the target, resulting in the reversible control of a receptor in timed manner driven by light. The most common photoswitchable core used is azobenzene. Azobenzene changes geometry during photoisomerization. In the dark or under white light, the azobenzene moiety is in a trans configuration converting to a cis configuration upon illumination with an appropriate wavelength (usually in the UV range). Relaxation to the thermodynamically more stable trans-isomer can be induced by irradiation or by thermal relaxation.

The first allosteric photoswitchable ligand targeting a GPCR is Alloswitch-1, an mGlu $_{5}$ receptor NAM (Pittolo et al., 2014). An azobenzene was inserted in the core of $\mathrm{N}$-(4-(2-chlorobenzamido)-3-methoxyphenyl) picolinamide, an $\mathrm{mGlu}_{4}$ receptor allosteric ligand having high chemical and structural homology with the 
scaffold present in azobenzene (Engers et al., 2011), to minimally modify the steric occupancy, binding determinants, and physicochemical properties of the parent compounds. Illumination by green or violet light stabilizes either the trans or the cis configuration of the ligand that corresponds to high and low pharmacological activity, respectively, on heterologous or native cells expressing the $\mathrm{mGlu}_{5}$ receptor. In vivo, Alloswitch-1 allows light-dependent control of the motility of Xenopus laevi tadpoles (Pittolo et al., 2014). More recently,

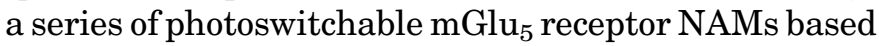
on the phenylazopyridine scaffold was generated (Gómez-Santacana et al., 2017). Most of the transisomers of this series are active both in vitro, inhibiting $\mathrm{mGlu}_{5}$ receptor function in heterologous cells, and in vivo, photocontrolling zebrafish motility. Optogluram is a derivative of Alloswitch-1, which acts as a photoswitchable $\mathrm{mGlu}_{4}$ receptor PAM, enabling for selective, reversible, and repeated optical manipulation of $\mathrm{mGlu}_{4}$ receptor activity (Zussy et al., 2018) (Fig. 10D). Optogluram allows the photocontrol of endogenous $\mathrm{mGlu}_{4}$ receptor activity in specific brain of freely behaving mice, revealing the dynamic control of pain-related sensory and anxiodepressive symptoms by amygdala $\mathrm{mGlu}_{4}$ receptors (Zussy et al., 2018). Since UV light could be potentially damaging to irradiated tissues, designing red-shifted photoswitchable ligands has been of considerable interest. Recently, OptoGluNAM4.1, a blue light-sensitive $\mathrm{mGlu}_{4}$ receptor photoswitchable NAM, was described that is active both in vitro and in vivo, photocontrolling zebrafish larvae mobility or blocking the analgesic activity of an $\mathrm{mGlu}_{4}$ receptor agonist in a mouse model of chronic pain (Rovira et al., 2016). Manipulating mGlu receptor with high spatial and temporal precision holds great promise for exploring physiologic and pathologic functions. As the field is rapidly evolving, the number of optical tools available will likely increase and provide new means to probe the biologic function of $\mathrm{mGlu}$ receptors.

\section{Conclusion}

From the initial identification of mGlu receptors in the early 1990 s, the past 30 years have seen rapid progress, with the discovery of novel pharmacological agents and application of chemical, genetic, and optical biology tools to dissect the molecular properties of the eight subtypes. Each of the individual subtypes offers promise as a potential therapeutic target. Excitingly, the intensive drug discovery efforts have led to multiple candidates reaching clinical trials with varied mechanisms of action. Both orthosteric and allosteric ligands offer considerable complexity in their biologic effects, with biased agonism/modulation, context, and probe dependence, coupled with additional complexity presented by mGlu receptor heteromers. Although it poses a challenge for discovery, harnessing this pharmacological and biologic complexity presents new opportunities to precisely tailor the activity of mGlu receptors to maximize therapeutic efficacy and avoid adverse effect liability.

\section{Authorship Contributions}

Wrote the manuscript and prepared figures: Gregory, Goudet.

\section{References}

Abd-Elrahman KS, Hamilton A, Hutchinson SR, Liu F, Russell RC, and Ferguson SSG (2017) mGluR5 antagonism increases autophagy and prevents disease progression in the $z Q 175$ mouse model of Huntington's disease. Sci Signal 10: eaan6387.

Abe M, Seto M, Gogliotti RG, Loch MT, Bollinger KA, Chang S, Engelberg EM, Luscombe VB, Harp JM, Bubser M, et al. (2017) Discovery of VU6005649, a CNS penetrant $\mathrm{mGlu}_{7 / 8}$ receptor PAM derived from a series of pyrazolo[1,5-a]pyrimidines. ACS Med Chem Lett 8:1110-1115.

Abe T, Sugihara H, Nawa H, Shigemoto R, Mizuno N, and Nakanishi S (1992) Molecular characterization of a novel metabotropic glutamate receptor mGluR5 coupled to inositol phosphate/Ca2+ signal transduction. J Biol Chem 267: 13361-13368.

Abitbol K, McLean H, Bessiron T, and Daniel H (2012) A new signalling pathway for parallel fibre presynaptic type 4 metabotropic glutamate receptors (mGluR4) in the rat cerebellar cortex. J Physiol 590:2977-2994

Abou Farha K, Bruggeman R, and Baljé-Volkers C (2014) Metabotropic glutamate receptor 5 negative modulation in phase I clinical trial: potential impact of circadian rhythm on the neuropsychiatric adverse reactions-do hallucinations matter? ISRN Psychiatry 2014:652750.

Achat-Mendes C, Platt DM, and Spealman RD (2012) Antagonism of metabotropic glutamate 1 receptors attenuates behavioral effects of cocaine and methamphetamine in squirrel monkeys. J Pharmacol Exp Ther 343:214-224.

Acher F, Battaglia G, Bräuner-Osborne H, Conn PJ, Duvoisin R, Ferraguti F, Flor PJ, Goudet C, Gregory KJ, Hampson D, et al. (2019) Metabotropic glutamate receptors (version 2019.4) in the IUPHAR/BPS Guide to Pharmacology Database. IUPHAR/BPS Guide to Pharmacology CITE 4 Available from: 10.2218/gtopdb/ F40/2019.4

Acher FC and Bertrand HO (2005) Amino acid recognition by Venus flytrap domains is encoded in an 8-residue motif. Biopolymers 80:357-366.

Acher FC, Selvam C, Pin JP, Goudet C, and Bertrand HO (2011) A critical pocket close to the glutamate binding site of mGlu receptors opens new possibilities for agonist design. Neuropharmacology 60:102-107.

Acher FC, Tellier FJ, Azerad R, Brabet IN, Fagni L, and Pin J-P (1997) Synthesis and pharmacological characterization of aminocyclopentanetricarboxylic acids: new tools to discriminate between metabotropic glutamate receptor subtypes. J Med Chem 40:3119-3129.

Acosta-Ruiz A, Gutzeit VA, Skelly MJ, Meadows S, Lee J, Parekh P, Orr AG, Liston C, Pleil KE, Broichhagen J, et al. (2020) Branched photoswitchable tethered ligands enable ultra-efficient optical control and detection of G protein-coupled receptors in vivo. Neuron 105:446-463.e13.

Adams DH, Kinon BJ, Baygani S, Millen BA, Velona I, Kollack-Walker S, and Walling DP (2013) A long-term, phase 2, multicenter, randomized, open-label, comparative safety study of pomaglumetad methionil (LY2140023 monohydrate) versus atypical antipsychotic standard of care in patients with schizophrenia. BMC Psychiatry 13:143.

Adams DH, Zhang L, Millen BA, Kinon BJ, and Gomez JC (2014) Pomaglumetad methionil (LY2140023 monohydrate) and aripiprazole in patients with schizophrenia: a phase 3, multicenter, double-blind comparison. Schizophr Res Treatment 2014:758212.

Ade KK, Wan Y, Hamann HC, O'Hare JK, Guo W, Quian A, Kumar S, Bhagat S, Rodriguiz RM, Wetsel WC, et al. (2016) Increased metabotropic glutamate receptor 5 signaling underlies obsessive-compulsive disorder-like behavioral and striatal circuit abnormalities in mice. Biol Psychiatry 80:522-533.

Ahnaou A, Dautzenberg FM, Geys H, Imogai H, Gibelin A, Moechars D, Steckler T, and Drinkenburg WH (2009) Modulation of group II metabotropic glutamate receptor (mGlu2) elicits common changes in rat and mice sleep-wake architecture. Eur J Pharmacol 603:62-72.

Ahnaou A, de Boer P, Lavreysen H, Huysmans H, Sinha V, Raeymaekers L, Van De Casteele T, Cid JM, Van Nueten L, Macdonald GJ, et al. (2016a) Translational neurophysiological markers for activity of the metabotropic glutamate receptor (mGluR2) modulator JNJ-40411813: sleep EEG correlates in rodents and healthy men. Neuropharmacology 103:290-305.

Ahnaou A, Lavreysen H, Tresadern G, Cid JM, and Drinkenburg WH (2015) mGlu2 receptor agonism, but not positive allosteric modulation, elicits rapid tolerance towards their primary efficacy on sleep measures in rats. PLoS One 10:e0144017. Ahnaou A, Raeyemaekers L, Huysmans H, and Drinkenburg WHIM (2016b) Offtarget potential of AMN082 on sleep EEG and related physiological variables: evidence from mGluR7 (-/-) mice. Behav Brain Res 311:287-297.

Alagarsamy S, Saugstad J, Warren L, Mansuy IM, Gereau RW IV, and Conn PJ (2005) NMDA-induced potentiation of mGluR5 is mediated by activation of protein phosphatase 2B/calcineurin. Neuropharmacology 49 (Suppl 1):135-145.

Alexander SPH, Christopoulos A, Davenport AP, Kelly E, Mathie A, Peters JA, Veale EL, Armstrong JF, Faccenda E, Harding SD, et al.; CGTP Collaborators (2019) THE CONCISE GUIDE TO PHARMACOLOGY 2019/20: G protein-coupled receptors. Br J Pharmacol 176 (Suppl 1):S21-S141.

Amalric M, Lopez S, Goudet C, Fisone G, Battaglia G, Nicoletti F, Pin JP, and Acher FC (2013) Group III and subtype 4 metabotropic glutamate receptor agonists: discovery and pathophysiological applications in Parkinson's disease. Neuropharmacology 66:53-64 
Ambrosi G, Armentero MT, Levandis G, Bramanti P, Nappi G, and Blandini F (2010) Effects of early and delayed treatment with an mGluR5 antagonist on motor impairment, nigrostriatal damage and neuroinflammation in a rodent model of Parkinson's disease. Brain Res Bull 82:29-38.

Anderson PM, Pinault D, O'Brien TJ, and Jones NC (2014) Chronic administration of antipsychotics attenuates ongoing and ketamine-induced increases in cortical $\gamma$ oscillations. Int $J$ Neuropsychopharmacol 17:1895-1904.

Annes WF, Long A, Witcher JW, Ayan-Oshodi MA, Knadler MP, Zhang W, Mitchell MI, Cornelissen K, and Hall SD (2015) Relative contributions of presystemic and systemic peptidases to oral exposure of a novel metabotropic glutamate $2 / 3 \mathrm{re}$ ceptor agonist (LY404039) after oral administration of prodrug pomaglumetad methionil (LY2140023). J Pharm Sci 104:207-214.

Annoura H, Fukunaga A, Uesugi M, Tatsuoka T, and Horikawa Y (1996) A novel class of antagonists for metabotropic glutamate receptors, 7-(hydroxyimino)cyclopropa[b]chromen-1a-carboxylates. Bioorg Med Chem Lett 6:763-766.

Ansah OB, Gonçalves L, Almeida A, and Pertovaara A (2009) Enhanced pronociception by amygdaloid group I metabotropic glutamate receptors in nerve-injured animals. Exp Neurol 216:66-74.

Antflick JE, Vetiska S, Baizer JS, Yao Y, Baker GB, and Hampson DR (2009) L-Serine-O-phosphate in the central nervous system. Brain Res 1300:1-13.

Anwyl R (1999) Metabotropic glutamate receptors: electrophysiological properties and role in plasticity. Brain Res Brain Res Rev 29:83-120.

Aramori I and Nakanishi S (1992) Signal transduction and pharmacological characteristics of a metabotropic glutamate receptor, mGluR1, in transfected $\mathrm{CHO}$ cells. Neuron 8:757-765.

Armentero MT, Fancellu R, Nappi G, Bramanti P, and Blandini F (2006) Prolonged blockade of NMDA or mGluR5 glutamate receptors reduces nigrostriatal degeneration while inducing selective metabolic changes in the basal ganglia circuitry in a rodent model of Parkinson's disease. Neurobiol Dis 22:1-9.

Aronica E, Gorter JA, Ijlst-Keizers H, Rozemuller AJ, Yankaya B, Leenstra S, and Troost D (2003) Expression and functional role of mGluR3 and mGluR5 in human astrocytes and glioma cells: opposite regulation of glutamate transporter proteins. Eur J Neurosci 17:2106-2118.

Arsova A, Møller TC, Vedel L, Hansen JL, Foster SR, Gregory KJ, and BräunerOsborne H (2020) Detailed In Vitro Pharmacological Characterization of Clinically Tested Negative Allosteric Modulators of the Metabotropic Glutamate Receptor 5. Mol Pharmacol 98 (1):49-60.

Attwell PJ, Singh Kent N, Jane DE, Croucher MJ, and Bradford HF (1998) Anticonvulsant and glutamate release-inhibiting properties of the highly potent metabotropic glutamate receptor agonist $\left(2 \mathrm{~S}, 2^{\prime} \mathrm{R}, \quad 3^{\prime} \mathrm{R}\right)-2-\left(2^{\prime}, 3^{\prime}\right.$-dicarboxycyclopropyl)glycine (DCG-IV). Brain Res 805:138-143.

Aubrey KR, Drew GM, Jeong HJ, Lau BK, and Vaughan CW (2017) Endocannabinoids control vesicle release mode at midbrain periaqueductal grey inhibitory synapses. J Physiol 595:165-178.

Augier E, Dulman RS, Rauffenbart C, Augier G, Cross AJ, and Heilig M (2016) The mGluR2 positive allosteric modulator, AZD8529, and cue-induced relapse to alcohol seeking in rats. Neuropsychopharmacology 41:2932-2940.

Ayoub MA, Angelicheva D, Vile D, Chandler D, Morar B, Cavanaugh JA, Visscher PM, Jablensky A, Pfleger KD, and Kalaydjieva L (2012) Deleterious GRM1 mutations in schizophrenia. PLoS One 7:e32849.

Bahi A, Fizia K, Dietz M, Gasparini F, and Flor PJ (2012) Pharmacological modulation of mGluR7 with AMN082 and MMPIP exerts specific influences on alcohol consumption and preference in rats. Addict Biol 17:235-247.

Bailey DB Jr., Berry-Kravis E, Wheeler A, Raspa M, Merrien F, Ricart J, Koumaras B, Rosenkranz G, Tomlinson M, von Raison F, et al. (2016) Mavoglurant in adolescents with fragile X syndrome: analysis of Clinical Global ImpressionImprovement source data from a double-blind therapeutic study followed by an open-label, long-term extension study. J Neurodev Disord 8:1.

Balschun D, Zuschratter W, and Wetzel W (2006) Allosteric enhancement of metabotropic glutamate receptor 5 function promotes spatial memory. Neuroscience 142:691-702.

Balu DT, Li Y, Takagi S, Presti KT, Ramikie TS, Rook JM, Jones CK, Lindsley CW, Conn PJ, Bolshakov VY, et al. (2016) An mGlu5-positive allosteric modulator rescues the neuroplasticity deficits in a genetic model of NMDA receptor hypofunction in schizophrenia. Neuropsychopharmacology 41:2052-2061.

Bao WL, Williams AJ, Faden AI, and Tortella FC (2001) Selective mGluR5 receptor antagonist or agonist provides neuroprotection in a rat model of focal cerebral ischemia. Brain Res 922:173-179.

Barton ME, Peters SC, and Shannon HE (2003) Comparison of the effect of glutamate receptor modulators in the $6 \mathrm{~Hz}$ and maximal electroshock seizure models. Epilepsy Res 56:17-26.

Barton ME and Shannon HE (2005) Behavioral and convulsant effects of the (S) enantiomer of the group I metabotropic glutamate receptor agonist 3,5-DHPG in mice. Neuropharmacology 48:779-787.

Battaglia G and Bruno V (2018) Metabotropic glutamate receptor involvement in the pathophysiology of amyotrophic lateral sclerosis: new potential drug targets for therapeutic applications. Curr Opin Pharmacol 38:65-71.

Battaglia G, Busceti CL, Molinaro G, Biagioni F, Storto M, Fornai F, Nicoletti F, and Bruno V (2004) Endogenous activation of mGlu5 metabotropic glutamate receptors contributes to the development of nigro-striatal damage induced by 1 methyl-4-phenyl-1,2,3,6-tetrahydropyridine in mice. $J$ Neurosci 24:828-835.

Battaglia G, Busceti CL, Molinaro G, Biagioni F, Traficante A, Nicoletti F, and Bruno V (2006) Pharmacological activation of mGlu4 metabotropic glutamate receptors reduces nigrostriatal degeneration in mice treated with 1-methyl-4-phenyl-1,2,3,6 tetrahydropyridine. $J$ Neurosci 26:7222-7229.

Battaglia G, Busceti CL, Pontarelli F, Biagioni F, Fornai F, Paparelli A, Bruno V, Ruggieri S, and Nicoletti F (2003) Protective role of group-II metabotropic glutamate receptors against nigro-striatal degeneration induced by 1-methyl-4-phenyl1,2,3,6-tetrahydropyridine in mice. Neuropharmacology 45:155-166.

Battaglia G, Fornai F, Busceti CL, Aloisi G, Cerrito F, De Blasi A, Melchiorri D, and Nicoletti F (2002) Selective blockade of mGlu5 metabotropic glutamate receptors is protective against methamphetamine neurotoxicity. $J$ Neurosci 22:2135-2141.
Battaglia G, Riozzi B, Bucci D, Di Menna L, Molinaro G, Pallottino S, Nicoletti F, and Bruno V (2015) Activation of mGlu3 metabotropic glutamate receptors enhances GDNF and GLT-1 formation in the spinal cord and rescues motor neurons in the SOD-1 mouse model of amyotrophic lateral sclerosis. Neurobiol Dis 74: 126-136.

Becker JA, Clesse D, Spiegelhalter C, Schwab Y, Le Merrer J, and Kieffer BL (2014) Autistic-like syndrome in mu opioid receptor null mice is relieved by facilitated mGluR4 activity. Neuropsychopharmacology 39:2049-2060.

Bellozi PMQ, Gomes GF, da Silva MCM, Lima IVA, Batista CRA, Carneiro Junior WO, Dória JG, Vieira ELM, Vieira RP, de Freitas RP, et al. (2019) A positive allosteric modulator of mGluR5 promotes neuroprotective effects in mouse models of Alzheimer's disease. Neuropharmacology 160:107785.

Benneyworth MA, Xiang Z, Smith RL, Garcia EE, Conn PJ, and Sanders-Bush E (2007) A selective positive allosteric modulator of metabotropic glutamate receptor subtype 2 blocks a hallucinogenic drug model of psychosis. Mol Pharmacol 72: 477-484.

Benquet P, Gee CE, and Gerber U (2002) Two distinct signaling pathways upregulate NMDA receptor responses via two distinct metabotropic glutamate receptor subtypes. J Neurosci 22:9679-9686.

Benvenga MJ, Chaney SF, Baez M, Britton TC, Hornback WJ, Monn JA, and Marek

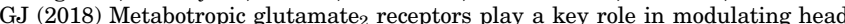
twitches induced by a serotonergic hallucinogen in mice. Front Pharmacol 9:208.

Beqollari D and Kammermeier PJ (2008) The mGlu(4) receptor allosteric modulator N-phenyl-7-(hydroxyimino)cyclopropa[b]chromen-1a-carboxamide acts as a direct agonist at $\mathrm{mGlu}(6)$ receptors. Eur $J$ Pharmacol 589:49-52.

Berizzi AE and Goudet C (2020) Strategies and considerations of G-protein-coupled receptor photopharmacology. Adv Pharmacol 88:143-172.

Berry-Kravis E, Des Portes V, Hagerman R, Jacquemont S, Charles P, Visootsak J, Brinkman M, Rerat K, Koumaras B, Zhu L, et al. (2016) Mavoglurant in fragile X syndrome: results of two randomized, double-blind, placebo-controlled trials. $S c i$ Transl Med 8:321ra5.

Berry-Kravis E, Hessl D, Coffey S, Hervey C, Schneider A, Yuhas J, Hutchison J, Snape M, Tranfaglia M, Nguyen DV, et al. (2009) A pilot open label, single dose trial of fenobam in adults with fragile X syndrome. J Med Genet 46:266-271.

Bertaso F, Zhang C, Scheschonka A, de Bock F, Fontanaud P, Marin P, Huganir RL, Betz H, Bockaert J, Fagni L, et al. (2008) PICK1 uncoupling from mGluR7a causes absence-like seizures. Nat Neurosci 11:940-948.

Bertrand HO, Bessis AS, Pin JP, and Acher FC (2002) Common and selective molecular determinants involved in metabotopic glutamate receptor agonist activity. J Med Chem 45:3171-3183.

Besheer J, Grondin JJ, Cannady R, Sharko AC, Faccidomo S, and Hodge CW (2010) Metabotropic glutamate receptor 5 activity in the nucleus accumbens is required for the maintenance of ethanol self-administration in a rat genetic model of high alcohol intake. Biol Psychiatry 67:812-822

Bessis AS, Bertrand HO, Galvez T, De Colle C, Pin JP, and Acher F (2000) Threedimensional model of the extracellular domain of the type $4 \mathrm{a}$ metabotropic glutamate receptor: new insights into the activation process. Protein Sci 9: 2200-2209.

Bessis AS, Rondard P, Gaven F, Brabet I, Triballeau N, Prezeau L, Acher F, and Pin JP (2002) Closure of the Venus flytrap module of $m$ Glu8 receptor and the activation process: Insights from mutations converting antagonists into agonists. Proc Nat Acad Sci USA 99:11097-11102.

Betts MJ, O'Neill MJ, and Duty S (2012) Allosteric modulation of the group III mGlu4 receptor provides functional neuroprotection in the 6-hydroxydopamine rat model of Parkinson's disease. Br J Pharmacol 166:2317-2330.

Beurrier C, Lopez S, Révy D, Selvam C, Goudet C, Lhérondel M, Gubellini P, Kerkerian-LeGoff L, Acher F, Pin JP, et al. (2009) Electrophysiological and behavioral evidence that modulation of metabotropic glutamate receptor 4 with a new agonist reverses experimental parkinsonism. FASEB J 23:3619-3628.

Bezard E, Pioli EY, Li Q, Girard F, Mutel V, Keywood C, Tison F, Rascol O, and Poli SM (2014) The mGluR5 negative allosteric modulator dipraglurant reduces dyskinesia in the MPTP macaque model. Mov Disord 29:1074-1079.

Bhattacharya M, Babwah AV, Godin C, Anborgh PH, Dale LB, Poulter MO, and Ferguson SS (2004) Ral and phospholipase D2-dependent pathway for constitutive metabotropic glutamate receptor endocytosis. J Neurosci 24:8752-8761.

Bhave G, Karim F, Carlton SM, and Gereau RW IV (2001) Peripheral group I metabotropic glutamate receptors modulate nociception in mice. Nat Neurosci 4: 417-423.

Bigge CF, Drummond JT, Johnson G, Malone T, Probert AW Jr., Marcoux FW, Coughenour LL, and Brahce LJ (1989) Exploration of phenyl-spaced 2-amino-(5-9)phosphonoalkanoic acids as competitive N-methyl-D-aspartic acid antagonists. J Med Chem 32:1580-1590.

Black JW and Leff P (1983) Operational models of pharmacological agonism. Proc $R$ Soc Lond B Biol Sci 220:141-162.

Black YD, Xiao D, Pellegrino D, Kachroo A, Brownell AL, and Schwarzschild MA (2010) Protective effect of metabotropic glutamate mGluR5 receptor elimination in a 6-hydroxydopamine model of Parkinson's disease. Neurosci Lett 486:161-165.

Blednov YA, Walker D, Osterndorf-Kahanek E, and Harris RA (2004) Mice lacking metabotropic glutamate receptor 4 do not show the motor stimulatory effect of ethanol. Alcohol 34:251-259.

Boccella S, Marabese I, Guida F, Luongo L, Maione S, and Palazzo E (2020) The modulation of pain by metabotropic glutamate receptors 7 and 8 in the dorsal striatum. Curr Neuropharmacol 18:34-50.

Bond A, Jones NM, Hicks CA, Whiffin GM, Ward MA, O'Neill MF, Kingston AE, Monn JA, Ornstein PL, Schoepp DD, et al. (2000) Neuroprotective effects of LY379268, a selective mGlu2/3 receptor agonist: investigations into possible mechanism of action in vivo. J Pharmacol Exp Ther 294:800-809.

Bond A, Monn JA, and Lodge D (1997) A novel orally active group 2 metabotropic glutamate receptor agonist: LY354740. Neuroreport 8:1463-1466.

Bond A, Ragumoorthy N, Monn JA, Hicks CA, Ward MA, Lodge D, and O'Neill MJ (1999) LY379268, a potent and selective Group II metabotropic glutamate receptor 
agonist, is neuroprotective in gerbil global, but not focal, cerebral ischaemia. Neurosci Lett 273:191-194.

Bordi F, Marcon C, Chiamulera C, and Reggiani A (1996) Effects of the metabotropic glutamate receptor antagonist MCPG on spatial and context-specific learning. Neuropharmacology 35:1557-1565.

Brabet I, Parmentier ML, De Colle C, Bockaert J, Acher F, and Pin JP (1998) Comparative effect of L-CCG-I, DCG-IV and gamma-carboxy-L-glutamate on all cloned metabotropic glutamate receptor subtypes. Neuropharmacology 37: 1043-1051.

Bradley SJ and Challiss RA (2011) Defining protein kinase/phosphatase isoenzymic

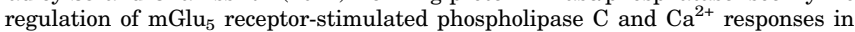
astrocytes. $\mathrm{Br} J$ Pharmacol 164:755-771.

Bradley SJ, Langmead CJ, Watson JM, and Challiss RA (2011) Quantitative analysis reveals multiple mechanisms of allosteric modulation of the mGlu5 receptor in rat astroglia. Mol Pharmacol 79:874-885.

Brody SA, Dulawa SC, Conquet F, and Geyer MA (2004) Assessment of a prepulse inhibition deficit in a mutant mouse lacking mGlu5 receptors. Mol Psychiatry 9: 35-41.

Broichhagen J, Damijonaitis A, Levitz J, Sokol KR, Leippe P, Konrad D, Isacoff EY, and Trauner D (2015) Orthogonal optical control of a G protein-coupled receptor with a SNAP-tethered photochromic ligand. ACS Cent Sci 1:383-393.

Bruno V, Battaglia G, Kingston A, O'Neill MJ, Catania MV, Di Grezia R, and Nicoletti F (1999) Neuroprotective activity of the potent and selective mGlu1a metabotropic glutamate receptor antagonist, (+)-2-methyl-4 carboxyphenylglycine (LY367385): comparison with LY357366, a broader spectrum antagonist with equal affinity for mGlu1a and mGlu5 receptors. Neuropharmacology 38:199-207.

Bruno V, Copani A, Battaglia G, Raffaele R, Shinozaki H, and Nicoletti F (1994) Protective effect of the metabotropic glutamate receptor agonist, DCG-IV, against excitotoxic neuronal death. Eur J Pharmacol 256:109-112.

Bruno V, Sureda FX, Storto M, Casabona G, Caruso A, Knopfel T, Kuhn R and Nicoletti F (1997) The neuroprotective activity of group-II metabotropic glutamate receptors requires new protein synthesis and involves a glial-neurona signaling. J Neurosci 17:1891-1897.

Burgueño J, Enrich C, Canela EI, Mallol J, Lluis C, Franco R, and Ciruela F (2003) Metabotropic glutamate type 1alpha receptor localizes in low-density caveolin-rich plasma membrane fractions. J Neurochem 86:785-791.

Cabello N, Gandía J, Bertarelli DC, Watanabe M, Lluís C, Franco R, Ferré S, Luján R, and Ciruela F (2009) Metabotropic glutamate type 5, dopamine D2 and adenosine A2a receptors form higher-order oligomers in living cells. J Neurochem 109:1497-1507.

Cai Z, Saugstad JA, Sorensen SD, Ciombor KJ, Zhang C, Schaffhauser H, Hubalek F, Pohl J, Duvoisin RM, and Conn PJ (2001) Cyclic AMP-dependent protein kinase phosphorylates group III metabotropic glutamate receptors and inhibits their function as presynaptic receptors. $J$ Neurochem 78:756-766.

Cain SM, Meadows HJ, Dunlop J, and Bushell TJ (2008) mGlu4 potentiation of K(2P) 2.1 is dependant on C-terminal dephosphorylation. Mol Cell Neurosci 37:32-39.

Callaway EM and Katz LC (1993) Photostimulation using caged glutamate reveals functional circuitry in living brain slices. Proc Natl Acad Sci USA 90:7661-7665.

Campo B, Kalinichev M, Lambeng N, El Yacoubi M, Royer-Urios I, Schneider M, Legrand C, Parron D, Girard F, Bessif A, et al. (2011) Characterization of an mGluR2/3 negative allosteric modulator in rodent models of depression. $J$ Neurogenet 25:152-166.

Capogna M (2004) Distinct properties of presynaptic group II and III metabotropic glutamate receptor-mediated inhibition of perforant pathway-CA1 EPSCs. Eur J Neurosci 19:2847-2858.

Caprioli D, Venniro M, Zeric T, Li X, Adhikary S, Madangopal R, Marchant NJ, Lucantonio F, Schoenbaum G, Bossert JM, et al. (2015) Effect of the novel positive allosteric modulator of metabotropic glutamate receptor 2 AZD8529 on incubation of methamphetamine craving after prolonged voluntary abstinence in a rat model. Biol Psychiatry 78:463-473.

Car H and Wiśniewska RJ (2006) Antidepressant-like effects of baclofen and LY367385 in the forced swim test in rats. Pharmacol Rep 58:758-764.

Caraci F, Molinaro G, Battaglia G, Giuffrida ML, Riozzi B, Traficante A, Bruno V, Cannella M, Merlo S, Wang X, et al. (2011) Targeting group II metabotropic glutamate (mGlu) receptors for the treatment of psychosis associated with Alzheimer's disease: selective activation of mGlu2 receptors amplifies beta-amyloid toxicity in cultured neurons, whereas dual activation of mGlu2 and mGlu3 receptors is neuroprotective. Mol Pharmacol 79:618-626.

Carroll EC, Berlin S, Levitz J, Kienzler MA, Yuan Z, Madsen D, Larsen DS, and Isacoff EY (2015) Two-photon brightness of azobenzene photoswitches designed for glutamate receptor optogenetics. Proc Natl Acad Sci USA 112:E776-E785.

Carroll FY, Stolle A, Beart PM, Voerste A, Brabet I, Mauler F, Joly C, Antonicek H, Bockaert J, Müller T, et al. (2001) BAY36-7620: a potent non-competitive mGlu1 receptor antagonist with inverse agonist activity. Mol Pharmacol 59:965-973.

Cartmell J, Adam G, Chaboz S, Henningsen R, Kemp JA, Klingelschmidt A, Metzler V, Monsma F, Schaffhauser H, Wichmann J, et al. (1998) Characterization of [3H] (2S,2'R,3'R)-2-(2',3'-dicarboxy-cyclopropyl)glycine ([3H]-DCG IV) binding to metabotropic mGlu2 receptor-transfected cell membranes. $\mathrm{Br} J$ Pharmacol 123 497-504.

Cartmell J, Monn JA, and Schoepp DD (1999) The metabotropic glutamate $2 / 3 \mathrm{re}$ ceptor agonists LY354740 and LY379268 selectively attenuate phencyclidine versus d-amphetamine motor behaviors in rats. J Pharmacol Exp Ther 291:161-170.

Cartmell J, Monn JA, and Schoepp DD (2000) Tolerance to the motor impairment, but not to the reversal of PCP-induced motor activities by oral administration of the mGlu2/3 receptor agonist, LY379268. Naunyn Schmiedebergs Arch Pharmacol 361:39-46.

Célanire S and Campo B (2012) Recent advances in the drug discovery of metabotropic glutamate receptor 4 (mGluR4) activators for the treatment of CNS and nonCNS disorders. Expert Opin Drug Discov 7:261-280.

Celanire S, Sebhat I, Wichmann J, Mayer S, Schann S, and Gatti S (2015) Novel metabotropic glutamate receptor $2 / 3$ antagonists and their therapeutic applications: a patent review (2005 - present). Expert Opin Ther Pat 25:69-90.
Chae E, Shin YJ, Ryu EJ, Ji MK, Ryune Cho N, Lee KH, Jeong HJ, Kim SJ, Choi Y, Seok Oh K, et al. (2013) Discovery of biological evaluation of pyrazole/imidazole amides as mGlu5 receptor negative allosteric modulators. Bioorg Med Chem Lett 23:2134-2139

Chaki S and Fukumoto K (2018) mGlu receptors as potential targets for novel antidepressants. Curr Opin Pharmacol 38:24-30.

Chapman AG, Nanan K, Williams M, and Meldrum BS (2000) Anticonvulsant activity of two metabotropic glutamate group I antagonists selective for the mGlu5 receptor: 2-methyl-6-(phenylethynyl)-pyridine (MPEP), and (E)-6-methyl-2-styrylpyridine (SIB 1893). Neuropharmacology 39:1567-1574.

Charvin D (2018) $\mathrm{mGlu}_{4}$ allosteric modulation for treating Parkinson's disease. Neuropharmacology 135:308-315.

Charvin D, Di Paolo T, Bezard E, Gregoire L, Takano A, Duvey G, Pioli E, Halldin C, Medori R, and Conquet F (2018a) An mGlu4-positive allosteric modulator alleviates Parkinsonism in primates. Mov Disord 33:1619-1631.

Charvin D, Medori R, Hauser RA, and Rascol O (2018b) Therapeutic strategies for Parkinson disease: beyond dopaminergic drugs. Nat Rev Drug Discov 17:804-822. Charvin D, Pomel V, Ortiz M, Frauli M, Scheffler S, Steinberg E, Baron L, Deshons L, Rudigier R, Thiarc D, et al. (2017) Discovery, structure-activity relationship, and antiparkinsonian effect of a potent and brain-penetrant chemical series of positive allosteric modulators of metabotropic glutamate receptor 4. J Med Chem 60 8515-8537.

Chaudhari N, Landin AM, and Roper SD (2000) A metabotropic glutamate receptor variant functions as a taste receptor. Nat Neurosci 3:113-119.

Chavis P, Mollard P, Bockaert J, and Manzoni O (1998) Visualization of cyclic AMPregulated presynaptic activity at cerebellar granule cells. Neuron 20:773-781.

Chen ANY, Hellyer SD, Trinh PNH, Leach K, and Gregory KJ (2020) Identification of monellin as the first naturally derived proteinaceous allosteric agonist of metabotropic glutamate receptor 5. Basic Clin Pharmacol Toxicol 126 (Suppl 6):104-115.

Chen SR and Pan HL (2005) Distinct roles of group III metabotropic glutamate receptors in control of nociception and dorsal horn neurons in normal and nerveinjured Rats. J Pharmacol Exp Ther 312:120-126.

Chen Y, Goudet C, Pin JP, and Conn PJ (2008) N-4-Chloro-2-[(1,3-dioxo-1,3-dihydro$2 \mathrm{H}$-isoindol-2-yl)methyl]phenyl-2-hydroxybenzamide (CPPHA) acts through a novel site as a positive allosteric modulator of group 1 metabotropic glutamate receptors. Mol Pharmacol 73:909-918.

Chen Y, Nong Y, Goudet C, Hemstapat K, de Paulis T, Pin JP, and Conn PJ (2007) Interaction of novel positive allosteric modulators of metabotropic glutamate receptor 5 with the negative allosteric antagonist site is required for potentiation of receptor responses. Mol Pharmacol 71:1389-1398.

Cheng J, Liu W, Duffney LJ, and Yan Z (2013) SNARE proteins are essential in the potentiation of NMDA receptors by group II metabotropic glutamate receptors. J Physiol 591:3935-3947.

Chiamulera C, Epping-Jordan MP, Zocchi A, Marcon C, Cottiny C, Tacconi S, Corsi M, Orzi F, and Conquet F (2001) Reinforcing and locomotor stimulant effects of cocaine are absent in mGluR5 null mutant mice. Nat Neurosci 4:873-874.

Cho HP, Engers DW, Venable DF, Niswender CM, Lindsley CW, Conn PJ, Emmitte $\mathrm{KA}$, and Rodriguez AL (2014a) A novel class of succinimide-derived negative allosteric modulators of metabotropic glutamate receptor subtype 1 provides insight into a disconnect in activity between the rat and human receptors. ACS Chem Neurosci 5:597-610.

Cho HP, Garcia-Barrantes PM, Brogan JT, Hopkins CR, Niswender CM, Rodriguez AL, Venable DF, Morrison RD, Bubser M, Daniels JS, et al. (2014b) Chemica modulation of mutant mGlu1 receptors derived from deleterious GRM1 mutations found in schizophrenics. ACS Chem Biol 9:2334-2346.

Chojnacka-Wójcik E, Tatarczyńska E, and Pilc A (1996) Anxiolytic-like effects of metabotropic glutamate antagonist (RS)-alpha-methylserine-O-phosphate in rats. Pol J Pharmacol 48:507-509.

Chojnacka-Wójcik E, Tatarczyńska E, and Pilc A (1997) The anxiolytic-like effect of metabotropic glutamate receptor antagonists after intrahippocampal injection in rats. Eur J Pharmacol 319:153-156.

Christoffersen GR, Christensen LH, Hammer P, and Vang M (1999) The class I metabotropic glutamate receptor antagonist, AIDA, improves short-term and impairs long-term memory in a spatial task for rats. Neuropharmacology 38 817-823.

Christopher JA, Aves SJ, Bennett KA, Doré AS, Errey JC, Jazayeri A, Marshall FH, Okrasa K, Serrano-Vega MJ, Tehan BG, et al. (2015) Fragment and structurebased drug discovery for a class C GPCR: discovery of the mGlu5 negative allosteric modulator HTL14242 (3-chloro-5-[6-(5-fluoropyridin-2-yl)pyrimidin-4-yl]benzonitrile). $J$ Med Chem 58:6653-6664.

Christopher JA, Orgován Z, Congreve M, Doré AS, Errey JC, Marshall FH, Mason JS, Okrasa K, Rucktooa P, Serrano-Vega MJ, et al. (2019) Structure-based optimization strategies for G protein-coupled receptor (GPCR) allosteric modulators: a case study from analyses of new metabotropic glutamate receptor $5\left(\mathrm{mGlu}_{5}\right)$ X-ray structures. J Med Chem 62:207-222.

Christov C, González-Bulnes P, Malhaire F, Karabencheva T, Goudet C, Pin JP, Llebaria A, and Giraldo J (2011) Integrated synthetic, pharmacological, and computational investigation of cis-2-(3,5-dichlorophenylcarbamoyl)cyclohexanecarboxylic acid enantiomers as positive allosteric modulators of metabotropic glutamate receptor subtype 4. ChemMedChem 6:131-140.

Ciccarelli R, D'Alimonte I, Ballerini P, D'Auro M, Nargi E, Buccella S, Di Iorio P, Bruno V, Nicoletti F, and Caciagli F (2007) Molecular signalling mediating the protective effect of $\mathrm{A} 1$ adenosine and mGlu3 metabotropic glutamate receptor activation against apoptosis by oxygen/glucose deprivation in cultured astrocytes. Mol Pharmacol 71:1369-1380.

Cid JM, Tresadern G, Duvey G, Lütjens R, Finn T, Rocher JP, Poli S, Vega JA, de Lucas AI, Matesanz E, et al. (2014) Discovery of 1-butyl-3-chloro-4-(4-phenyl-1piperidinyl)-(1H)-pyridone (JNJ-40411813): a novel positive allosteric modulator of the metabotropic glutamate 2 receptor. J Med Chem 57:6495-6512.

Cid JM, Tresadern G, Vega JA, de Lucas AI, Matesanz E, Iturrino L, Linares ML Garcia A, Andrés JI, Macdonald GJ, et al. (2012) Discovery of 3-cyclopropylmethyl- 
7-(4-phenylpiperidin-1-yl)-8-trifluoromethyl[1,2,4]triazolo[4,3-a]pyridine (JNJ42153605): a positive allosteric modulator of the metabotropic glutamate 2 receptor. J Med Chem 55:8770-8789.

Cieślik P, Woźniak M, Kaczorowska K, Brański P, Burnat G, Chocyk A, Bobula B, Gruca P, Litwa E, Pałucha-Poniewiera A, et al. (2018) Negative allosteric modulators of $\mathrm{mGlu}_{7}$ receptor as putative antipsychotic drugs. Front Mol Neurosci 11:316.

Cippitelli A, Damadzic R, Frankola K, Goldstein A, Thorsell A, Singley E, Eskay RL, and Heilig M (2010) Alcohol-induced neurodegeneration, suppression of transforming growth factor-beta, and cognitive impairment in rats: prevention by group II metabotropic glutamate receptor activation. Biol Psychiatry 67:823-830.

Ciruela F, Escriche M, Burgueno J, Angulo E, Casado V, Soloviev MM, Canela EI, Mallol J, Chan WY, Lluis C, et al. (2001) Metabotropic glutamate 1alpha and adenosine A1 receptors assemble into functionally interacting complexes. J Biol Chem 276:18345-18351.

Clark BP, Baker SR, Goldsworthy J, Harris JR, and Kingston AE (1997) (+)-2Methyl-4-carboxyphenylglycine (LY367385) selectively antagonises metabotropic glutamate mGluR1 receptors. Bioorg Med Chem Lett 7:2777-2780.

Clark M, Johnson BG, Wright RA, Monn JA, and Schoepp DD (2002) Effects of the mGlu2/3 receptor agonist LY379268 on motor activity in phencyclidine-sensitized rats. Pharmacol Biochem Behav 73:339-346.

Cleva RM, Hicks MP, Gass JT, Wischerath KC, Plasters ET, Widholm JJ, and Olive MF (2011) mGluR5 positive allosteric modulation enhances extinction learning following cocaine self-administration. Behav Neurosci 125:10-19.

Cleva RM, Watterson LR, Johnson MA, and Olive MF (2012) Differential modulation of thresholds for intracranial self-stimulation by mGlu5 positive and negative allosteric modulators: implications for effects on drug self-administration. Front Pharmacol 2:93.

Clifton NE, Morisot N, Girardon S, Millan MJ, and Loiseau F (2013) Enhancement of social novelty discrimination by positive allosteric modulators at metabotropic glutamate 5 receptors: adolescent administration prevents adult-onset deficits induced by neonatal treatment with phencyclidine. Psychopharmacology (Berl) 225:579-594.

Commare B, Rigault D, Lemasson IA, Deschamps P, Tomas A, Roussel P, Brabet I, Goudet C, Pin JP, Leroux FR, et al. (2015) Determination of the absolute configuration of phosphinic analogues of glutamate. Org Biomol Chem 13:1106-1112.

Conde-Ceide S, Martínez-Viturro CM, Alcázar J, Garcia-Barrantes PM, Lavreysen H, Mackie C, Vinson PN, Rook JM, Bridges TM, Daniels JS, et al. (2015) Discovery of VU0409551/JNJ-46778212: an mGlu5 positive allosteric modulator clinical candidate targeting schizophrenia. ACS Med Chem Lett 6:716-720.

Conti P, De Amici M, Bräuner-Osborne H, Madsen U, Toma L, and De Micheli C (2002) Synthesis and pharmacology of 3-hydroxy-delta2-isoxazoline-cyclopentane analogues of glutamic acid. Farmaco 57:889-895.

Copani A, Bruno V, Battaglia G, Leanza G, Pellitteri R, Russo A, Stanzani S, and Nicoletti F (1995) Activation of metabotropic glutamate receptors protects cultured neurons against apoptosis induced by beta-amyloid peptide. Mol Pharmacol 47:890-897.

Corti C, Aldegheri L, Somogyi P, and Ferraguti F (2002) Distribution and synaptic localisation of the metabotropic glutamate receptor 4 (mGluR4) in the rodent CNS. Neuroscience 110:403-420.

Corti C, Battaglia G, Molinaro G, Riozzi B, Pittaluga A, Corsi M, Mugnaini M, Nicoletti F, and Bruno V (2007) The use of knock-out mice unravels distinct roles for mGlu2 and mGlu3 metabotropic glutamate receptors in mechanisms of neurodegeneration/neuroprotection. J Neurosci 27:8297-8308.

Corti C, Restituito S, Rimland JM, Brabet I, Corsi M, Pin JP, and Ferraguti F (1998) Cloning and characterization of alternative mRNA forms for the rat metabotropic glutamate receptors mGluR7 and mGluR8. Eur J Neurosci 10:3629-3641.

Cosford ND, Roppe J, Tehrani L, Schweiger EJ, Seiders TJ, Chaudary A, Rao S, and Varney MA (2003) [3H]-methoxymethyl-MTEP and [3H]-methoxy-PEPy: potent and selective radioligands for the metabotropic glutamate subtype 5 (mGlu5) receptor. Bioorg Med Chem Lett 13:351-354.

Crawford JH, Wootton JF, Seabrook GR, and Scott RH (1997) Activation of Ca2+dependent currents in dorsal root ganglion neurons by metabotropic glutamate receptors and cyclic ADP-ribose precursors. J Neurophysiol 77:2573-2584.

Cross AJ, Anthenelli R, and Li X (2018) Metabotropic glutamate receptors 2 and 3 as targets for treating nicotine addiction. Biol Psychiatry 83:947-954.

Crupi R, Impellizzeri D, and Cuzzocrea S (2019) Role of metabotropic glutamate receptors in neurological disorders. Front Mol Neurosci 12:20.

Cryan JF, Kelly PH, Neijt HC, Sansig G, Flor PJ, and van Der Putten H (2003) Antidepressant and anxiolytic-like effects in mice lacking the group III metabotropic glutamate receptor mGluR7. Eur J Neurosci 17:2409-2417.

Cuomo D, Martella G, Barabino E, Platania P, Vita D, Madeo G, Selvam C, Goudet C, Oueslati N, Pin JP, et al. (2009) Metabotropic glutamate receptor subtype 4 selectively modulates both glutamate and GABA transmission in the striatum: implications for Parkinson's disease treatment. J Neurochem 109:1096-1105.

Dale LB, Bhattacharya M, Anborgh PH, Murdoch B, Bhatia M, Nakanishi S, and Ferguson SS (2000) G protein-coupled receptor kinase-mediated desensitization of metabotropic glutamate receptor 1A protects against cell death. J Biol Chem 275:38213-38220.

Dalton JAR, Pin JP, and Giraldo J (2017) Analysis of positive and negative allosteric modulation in metabotropic glutamate receptors 4 and 5 with a dual ligand. Sci Rep 7:4944.

D'Amore V, Raaijmakers RH, Santolini I, van Rijn CM, Ngomba RT, Nicoletti F, and van Luijtelaar G (2016) The anti-absence effect of mGlu5 receptor amplification with VU0360172 is maintained during and after antiepileptogenesis. Phar macol Biochem Behav 146-147:50-59.

D'Amore V, Santolini I, Celli R, Lionetto L, De Fusco A, Simmaco M, van Rijn CM, Vieira E, Stauffer SR, Conn PJ, et al. (2014) Head-to head comparison of mGlu1 and mGlu5 receptor activation in chronic treatment of absence epilepsy in WAG/Rij rats. Neuropharmacology 85:91-103.

D'Amore V, Santolini I, van Rijn CM, Biagioni F, Molinaro G, Prete A, Conn PJ, Lindsley CW, Zhou Y, Vinson PN, et al. (2013) Potentiation of mGlu5 receptors with the novel enhancer, VU0360172, reduces spontaneous absence seizures in WAG/Rij rats. Neuropharmacology 66:330-338

D'Amore V, von Randow C, Nicoletti F, Ngomba RT, and van Luijtelaar G (2015) Anti-absence activity of mGlu1 and mGlu5 receptor enhancers and their interaction with a GABA reuptake inhibitor: effect of local infusions in the somatosensory cortex and thalamus. Epilepsia 56:1141-1151.

de Esch CE, van den Berg WE, Buijsen RA, Jaafar IA, Nieuwenhuizen-Bakker IM, Gasparini F, Kushner SA, and Willemsen R (2015) Fragile X mice have robust mGluR5-dependent alterations of social behaviour in the Automated Tube Test. Neurobiol Dis 75:31-39.

Dekundy A, Gravius A, Hechenberger M, Pietraszek M, Nagel J, Tober C, van der Elst M, Mela F, Parsons CG, and Danysz W (2011) Pharmacological characterization of MRZ-8676, a novel negative allosteric modulator of subtype 5 metabotropic glutamate receptors (mGluR5): focus on L: -DOPA-induced dyskinesia. $J$ Neural Transm (Vienna) 118:1703-1716.

Delille HK, Mezler M, and Marek GJ (2013) The two faces of the pharmacological interaction of mGlu2 and $5-\mathrm{HT}_{2} \mathrm{~A}$ - relevance of receptor heterocomplexes and interaction through functional brain pathways. Neuropharmacology 70:296-305.

de Paulis T, Hemstapat K, Chen Y, Zhang Y, Saleh S, Alagille D, Baldwin RM, Tamagnan GD, and Conn PJ (2006) Substituent effects of N-(1,3-diphenyl-1Hpyrazol-5-yl)benzamides on positive allosteric modulation of the metabotropic glutamate-5 receptor in rat cortical astrocytes. J Med Chem 49:3332-3344.

De Vry J, Horváth E, and Schreiber R (2001) Neuroprotective and behavioral effects of the selective metabotropic glutamate mGlu(1) receptor antagonist BAY 36-7620. Eur J Pharmacol 428:203-214.

Díaz-Cabiale Z, Vivó M, Del Arco A, O’Connor WT, Harte MK, Müller CE, Martínez E, Popoli P, Fuxe K, and Ferré S (2002) Metabotropic glutamate mGlu5 receptor-mediated modulation of the ventral striopallidal GABA pathway in rats. Interactions with adenosine $\mathrm{A}(2 \mathrm{~A})$ and dopamine $\mathrm{D}(2)$ receptors. Neurosci Lett 324:154-158.

Di Menna L, Joffe ME, Iacovelli L, Orlando R, Lindsley CW, Mairesse J, Gressèns P, Cannella M, Caraci F, Copani A, et al. (2018) Functional partnership between mGlu3 and mGlu5 metabotropic glutamate receptors in the central nervous system. Neuropharmacology 128:301-313.

DiRaddo JO, Miller EJ, Bowman-Dalley C, Wroblewska B, Javidnia M, Grajkowska E, Wolfe BB, Liotta DC, and Wroblewski JT (2015) Chloride is an agonist of group II and III metabotropic glutamate receptors. Mol Pharmacol 88:450-459.

DiRaddo JO, Pshenichkin S, Gelb T, and Wroblewski JT (2013) Two newly identified exons in human GRM1 express a novel splice variant of metabotropic glutamate 1 receptor. Gene 519:367-373.

Doherty AJ, Palmer MJ, Henley JM, Collingridge GL, and Jane DE (1997) (RS)-2chloro-5-hydroxyphenylglycine (CHPG) activates mGlu5, but no mGlu1, receptors expressed in CHO cells and potentiates NMDA responses in the hippocampus. Neuropharmacology 36:265-267.

Dölen G and Bear MF (2008) Role for metabotropic glutamate receptor 5 (mGluR5) in the pathogenesis of fragile X syndrome. J Physiol 586:1503-1508.

Domenici MR, Pepponi R, Martire A, Tebano MT, Potenza RL, and Popoli P (2004) Permissive role of adenosine $\mathrm{A} 2 \mathrm{~A}$ receptors on metabotropic glutamate receptor 5 (mGluR5)-mediated effects in the striatum. J Neurochem 90:1276-1279.

Domin H, Gołembiowska K, Jantas D, Kamińska K, Zięba B, and Smiałowska M (2014) Group III mGlu receptor agonist, ACPT-I, exerts potential neuroprotective effects in vitro and in vivo. Neurotox Res 26:99-113.

Domin H, Przykaza Ł, Jantas D, Kozniewska E, Boguszewski PM, and Śmiałowska M (2016) Neuroprotective potential of the group III mGlu receptor agonist ACPT-I in animal models of ischemic stroke: in vitro and in vivo studies. Neuropharmacology 102:276-294.

Domin H, Przykaza Ł, Kozniewska E, Boguszewski PM, and Śmiałowska M (2018) Neuroprotective effect of the group III mGlu receptor agonist ACPT-I after ischemic stroke in rats with essential hypertension. Prog Neuropsychopharmacol Biol Psychiatry 84:93-101.

Donthamsetti PC, Broichhagen J, Vyklicky V, Stanley C, Fu Z, Visel M, Levitz JL, Javitch JA, Trauner D, and Isacoff EY (2019) Genetically targeted optical control of an endogenous G protein-coupled receptor. J Am Chem Soc 141: $11522-11530$

Doornbos ML, Pérez-Benito L, Tresadern G, Mulder-Krieger T, Biesmans I, Trabanco AA, Cid JM, Lavreysen H, IJzerman AP, and Heitman LH (2016) Molecular mechanism of positive allosteric modulation of the metabotropic glutamate receptor 2 by JNJ-46281222. Br J Pharmacol 173:588-600.

Doornbos MLJ, Cid JM, Haubrich J, Nunes A, van de Sande JW, Vermond SC, Mulder-Krieger T, Trabanco AA, Ahnaou A, Drinkenburg WH, et al. (2017) Discovery and kinetic profiling of 7-Aryl-1,2,4-triazolo[4,3-a]pyridines: positive allosteric modulators of the metabotropic glutamate receptor $2 . J \mathrm{Med}$ Chem 60: $6704-6720$.

Doornbos MLJ, Wang X, Vermond SC, Peeters L, Pérez-Benito L, Trabanco AA, Lavreysen H, Cid JM, Heitman LH, Tresadern G, et al. (2019) Covalent allosteric probe for the metabotropic glutamate receptor 2 : design, synthesis, and pharmacological characterization. J Med Chem 62:223-233.

Doré AS, Okrasa K, Patel JC, Serrano-Vega M, Bennett K, Cooke RM, Errey JC, Jazayeri A, Khan S, Tehan B, et al. (2014) Structure of class C GPCR metabotropic glutamate receptor 5 transmembrane domain. Nature 511:557-562.

Doria JG, de Souza JM, Andrade JN, Rodrigues HA, Guimaraes IM, Carvalho TG, Guatimosim C, Dobransky T, and Ribeiro FM (2015) The mGluR5 positive allosteric modulator, CDPPB, ameliorates pathology and phenotypic signs of a mouse model of Huntington's disease. Neurobiol Dis 73:163-173.

Doria JG, de Souza JM, Silva FR, Olmo IG, Carvalho TG, Alves-Silva J, FerreiraVieira TH, Santos JT, Xavier CQS, Silva NC, et al. (2018) The mGluR5 positive allosteric modulator VU0409551 improves synaptic plasticity and memory of a mouse model of Huntington's disease. J Neurochem 147:222-239.

Doria JG, Silva FR, de Souza JM, Vieira LB, Carvalho TG, Reis HJ, Pereira GS, Dobransky T, and Ribeiro FM (2013) Metabotropic glutamate receptor 5 positive allosteric modulators are neuroprotective in a mouse model of Huntington's disease. Br J Pharmacol 169:909-921. 
Doumazane E, Scholler P, Fabre L, Zwier JM, Trinquet E, Pin JP, and Rondard P (2013) Illuminating the activation mechanisms and allosteric properties of metabotropic glutamate receptors. Proc Natl Acad Sci USA 110:E1416-E1425.

Doumazane E, Scholler P, Zwier JM, Trinquet E, Rondard P, and Pin JP (2011) A new approach to analyze cell surface protein complexes reveals specific heterodimeric metabotropic glutamate receptors. FASEB J 25:66-77.

Downing AM, Kinon BJ, Millen BA, Zhang L, Liu L, Morozova MA, Brenner R, Rayle TJ, Nisenbaum L, Zhao F, et al. (2014) A double-blind, placebo-controlled comparator study of LY2140023 monohydrate in patients with schizophrenia. BMC Psychiatry 14:351.

Dravolina OA, Zvartau E, Danysz W, and Bespalov AY (2017) mGlu1 receptor as a drug target for treatment of substance use disorders: time to gather stones together? Psychopharmacology (Berl) 234:1333-1345.

Dryja TP, McGee TL, Berson EL, Fishman GA, Sandberg MA, Alexander KR, Derlacki DJ, and Rajagopalan AS (2005) Night blindness and abnormal cone electroretinogram ON responses in patients with mutations in the GRM6 gene encoding mGluR6. Proc Natl Acad Sci USA 102:4884-4889.

Dunayevich E, Erickson J, Levine L, Landbloom R, Schoepp DD, and Tollefson GD (2008) Efficacy and tolerability of an mGlu2/3 agonist in the treatment of generalized anxiety disorder. Neuropsychopharmacology 33:1603-1610.

Durand D, Carniglia L, Caruso C, and Lasaga M (2011) Reduced cAMP, Akt activation and p65-c-Rel dimerization: mechanisms involved in the protective effects of mGluR3 agonists in cultured astrocytes. PLoS One 6:e22235.

Dutar P, Vu HM, and Perkel DJ (1999) Pharmacological characterization of an unusual mGluR-evoked neuronal hyperpolarization mediated by activation of GIRK channels. Neuropharmacology 38:467-475.

Duvoisin RM, Pfankuch T, Wilson JM, Grabell J, Chhajlani V, Brown DG, Johnson E, and Raber J (2010) Acute pharmacological modulation of mGluR8 reduces measures of anxiety. Behav Brain Res 212:168-173.

Duvoisin RM, Villasana L, Davis MJ, Winder DG, and Raber J (2011) Opposing roles of mGluR8 in measures of anxiety involving non-social and social challenges. Behav Brain Res 221:50-54.

Duvoisin RM, Zhang C, Pfankuch TF, O'Connor H, Gayet-Primo J, Quraishi S, and Raber J (2005) Increased measures of anxiety and weight gain in mice lacking the group III metabotropic glutamate receptor mGluR8. Eur $J$ Neurosci 22 425-436.

Duvoisin RM, Zhang C, and Ramonell K (1995) A novel metabotropic glutamate receptor expressed in the retina and olfactory bulb. $J$ Neurosci 15:3075-3083.

East SP and Gerlach K (2010) mGluR4 positive allosteric modulators with potential for the treatment of Parkinson's disease: WO09010455. Expert Opin Ther Pat 20: $441-445$.

Elia J, Ungal G, Kao C, Ambrosini A, De Jesus-Rosario N, Larsen L, Chiavacci R, Wang T, Kurian C, Titchen K, et al. (2018) Fasoracetam in adolescents with ADHD and glutamatergic gene network variants disrupting mGluR neurotransmitter signaling. Nat Commun 9:4.

El-Kouhen O, Lehto SG, Pan JB, Chang R, Baker SJ, Zhong C, Hollingsworth PR, Mikusa JP, Cronin EA, Chu KL, et al. (2006) Blockade of mGluR1 receptor results in analgesia and disruption of motor and cognitive performances: effects of A841720, a novel non-competitive mGluR1 receptor antagonist. $B r$ J Pharmacol 149 : 761-774.

El Moustaine D, Granier S, Doumazane E, Scholler P, Rahmeh R, Bron P, Mouillac B, Banères JL, Rondard P, and Pin JP (2012) Distinct roles of metabotropic glutamate receptor dimerization in agonist activation and G-protein coupling. Proc Natl Acad Sci USA 109:16342-16347.

Emery AC, Diraddo JO, Miller E, Hathaway HA, Pshenichkin S, Takoudjou GR, Grajkowska E, Yasuda RP, Wolfe BB, and Wroblewski J (2012) Ligand bias at metabotropic glutamate 1a receptors: molecular determinants that distinguish $\beta$-arrestin-mediated from G protein-mediated signaling. Mol Pharmacol 82: 291-301.

Emery AC, Pshenichkin S, Takoudjou GR, Grajkowska E, Wolfe BB, and Wroblewski JT (2010) The protective signaling of metabotropic glutamate receptor 1 is mediated by sustained, beta-arrestin-1-dependent ERK phosphorylation. $J$ Biol Chem 285:26041-26048.

Emile L, Mercken L, Apiou F, Pradier L, Bock MD, Menager J, Clot J, Doble A, and Blanchard JC (1996) Molecular cloning, functional expression, pharmacological characterization and chromosomal localization of the human metabotropic glutamate receptor type 3. Neuropharmacology 35:523-530.

Eng AG, Kelver DA, Hedrick TP, and Swanson GT (2016) Transduction of group I mGluR-mediated synaptic plasticity by $\beta$-arrestin 2 signalling. Nat Commun 7 : 13571.

Engers DW, Blobaum AL, Gogliotti RD, Cheung YY, Salovich JM, Garcia-Barrantes PM, Daniels JS, Morrison R, Jones CK, Soars MG, et al. (2016) Discovery, synthesis, and preclinical characterization of $\mathrm{N}$-(3-chloro-4-fluorophenyl)-1H-pyrazolo [4,3-b]pyridin-3-amine (VU0418506), a novel positive allosteric modulator of the metabotropic glutamate receptor 4 (mGlu4). ACS Chem Neurosci 7:1192-1200.

Engers DW, Field JR, Le U, Zhou Y, Bolinger JD, Zamorano R, Blobaum AL, Jones CK, Jadhav S, Weaver CD, et al. (2011) Discovery, synthesis, and structure-activity relationship development of a series of $\mathrm{N}$-(4-acetamido)phenylpicolinamides as positive allosteric modulators of metabotropic glutamate receptor 4 (mGlu(4)) with CNS exposure in rats. $J$ Med Chem 54:1106-1110.

Engers DW, Gentry PR, Williams R, Bolinger JD, Weaver CD, Menon UN, Conn PJ, Lindsley CW, Niswender CM, and Hopkins CR (2010) Synthesis and SAR of novel, 4-(phenylsulfamoyl)phenylacetamide mGlu4 positive allosteric modulators (PAMs) identified by functional high-throughput screening (HTS). Bioorg Med Chem Lett 20:5175-5178

Engers DW, Niswender CM, Weaver CD, Jadhav S, Menon UN, Zamorano R, Conn PJ, Lindsley CW, and Hopkins CR (2009) Synthesis and evaluation of a series of heterobiarylamides that are centrally penetrant metabotropic glutamate receptor 4 (mGluR4) positive allosteric modulators (PAMs). J Med Chem 52:4115-4118.

Engers JL, Bollinger KA, Weiner RL, Rodriguez AL, Long MF, Breiner MM, Chang S, Bollinger SR, Bubser M, Jones CK, et al. (2017) Design and synthesis of $N$-aryl phenoxyethoxy pyridinones as highly selective and CNS penetrant mGlu $\mathrm{NAMs}_{3}$ ACS Med Chem Lett 8:925-930.

Engers JL, Rodriguez AL, Konkol LC, Morrison RD, Thompson AD, Byers FW, Blobaum AL, Chang S, Venable DF, Loch MT, et al. (2015) Discovery of a selective and CNS penetrant negative allosteric modulator of metabotropic glutamate receptor subtype 3 with antidepressant and anxiolytic activity in rodents. $J \mathrm{Med}$ Chem 58:7485-7500.

Erdmann E, Rupprecht V, Matthews E, Kukley M, Schoch S, and Dietrich D (2012) Depression of release by mGluR8 alters $\mathrm{Ca} 2+$ dependence of release machinery. Cereb Cortex 22:1498-1509.

Faden AI, Ivanova SA, Yakovlev AG, and Mukhin AG (1997) Neuroprotective effects of group III mGluR in traumatic neuronal injury. J Neurotrauma 14:885-895.

Faden AI, O'Leary DM, Fan L, Bao W, Mullins PG, and Movsesyan VA (2001) Selective blockade of the mGluR1 receptor reduces traumatic neuronal injury in vitro and improvesoOutcome after brain trauma. Exp Neurol 167:435-444

Fallarino F, Volpi C, Fazio F, Notartomaso S, Vacca C, Busceti C, Bicciato S, Battaglia G, Bruno V, Puccetti P, et al. (2010) Metabotropic glutamate receptor-4 modulates adaptive immunity and restrains neuroinflammation. Nat Med 16:897-902.

Farinha A, Lavreysen H, Peeters L, Russo B, Masure S, Trabanco AA, Cid J, and Tresadern G (2015) Molecular determinants of positive allosteric modulation of the human metabotropic glutamate receptor 2. Br J Pharmacol 172:2383-2396.

Farrants H, Gutzeit VA, Acosta-Ruiz A, Trauner D, Johnsson K, Levitz J, and Broichhagen J (2018) SNAP-tagged nanobodies enable reversible optical control of a $\mathrm{G}$ protein-coupled receptor via a remotely tethered photoswitchable ligand. ACS Chem Biol 13:2682-2688.

Fazio F, Lionetto L, Curto M, Iacovelli L, Copeland CS, Neale SA, Bruno V, Battaglia G, Salt TE, and Nicoletti F (2017) Cinnabarinic acid and xanthurenic acid: two kynurenine metabolites that interact with metabotropic glutamate receptors. Neuropharmacology 112:365-372.

Fazio F, Lionetto L, Molinaro G, Bertrand HO, Acher F, Ngomba RT, Notartomaso S, Curini M, Rosati O, Scarselli P, et al. (2012) Cinnabarinic acid, an endogenous metabolite of the kynurenine pathway, activates type 4 metabotropic glutamate receptors. Mol Pharmacol 81:643-656.

Fazio F, Zappulla C, Notartomaso S, Busceti C, Bessede A, Scarselli P, Vacca C, Gargaro M, Volpi C, Allegrucci M, et al. (2014) Cinnabarinic acid, an endogenous agonist of type-4 metabotropic glutamate receptor, suppresses experimental autoimmune encephalomyelitis in mice. Neuropharmacology 81:237-243.

Feenstra MG, Botterblom MH, and van Uum JF (1998) Local activation of metabotropic glutamate receptors inhibits the handling-induced increased release of dopamine in the nucleus accumbens but not that of dopamine or noradrenaline in the prefrontal cortex: comparison with inhibition of ionotropic receptors. J Neurochem 70:1104-1113.

Felder CC, Schober DA, Tu Y, Quets A, Xiao H, Watt M, Siuda E, Nisenbaum E, Xiang C, Heinz B, et al. (2017) Translational pharmacology of the metabotropic glutamate 2 receptor-preferring agonist LY2812223 in the animal and human brain. J Pharmacol Exp Ther 361:190-197.

Fell MJ, Katner JS, Johnson BG, Khilevich A, Schkeryantz JM, Perry KW, and Svensson KA (2010) Activation of metabotropic glutamate (mGlu)2 receptors suppresses histamine release in limbic brain regions following acute ketamine challenge. Neuropharmacology 58:632-639.

Fell MJ, Svensson KA, Johnson BG, and Schoepp DD (2008) Evidence for the role of metabotropic glutamate (mGlu) 2 not mGlu3 receptors in the preclinical antipsychotic pharmacology of the mGlu2/3 receptor agonist (-)-(1R,4S,5S,6S)-4-amino-2sulfonylbicyclo[3.1.0] hexane-4,6-dicarboxylic acid (LY404039). J Pharmacol Exp Ther 326:209-217.

Felsing DE, Anastasio NC, Miszkiel JM, Gilbertson SR, Allen JA, and Cunningham KA (2018) Biophysical validation of serotonin 5-HT2A and 5-HT2C receptor interaction. PLoS One 13:e0203137.

Felts AS, Rodriguez AL, Morrison RD, Venable DF, Manka JT, Bates BS, Blobaum AL, Byers FW, Daniels JS, Niswender CM, et al. (2013) Discovery of VU0409106: a negative allosteric modulator of mGlu 5 with activity in a mouse model of anxiety. Bioorg Med Chem Lett 23:5779-5785.

Felts AS, Rodriguez AL, Smith KA, Engers JL, Morrison RD, Byers FW, Blobaum AL, Locuson CW, Chang S, Venable DF, et al. (2015) Design of 4-Oxo-1-aryl-1,4dihydroquinoline-3-carboxamides as selective negative allosteric modulators of metabotropic glutamate receptor subtype 2. J Med Chem 58:9027-9040.

Fendt M, Bürki H, Imobersteg S, van der Putten H, McAllister K, Leslie JC, Shaw D, and Hölscher C (2010) The effect of mGlu8 deficiency in animal models of psychiatric diseases. Genes Brain Behav 9:33-44.

Fendt M, Imobersteg S, Peterlik D, Chaperon F, Mattes C, Wittmann C, Olpe HR, Mosbacher J, Vranesic I, van der Putten H, et al. (2013) Differential roles of $\mathrm{mGlu}(7)$ and mGlu(8) in amygdala-dependent behavior and physiology. Neuropharmacology 72:215-223.

Ferraguti F (2018) Metabotropic glutamate receptors as targets for novel anxiolytics. Curr Opin Pharmacol 38:37-42.

Ferraguti F, Klausberger T, Cobden P, Baude A, Roberts JD, Szucs P, Kinoshita A, Shigemoto R, Somogyi P, and Dalezios Y (2005) Metabotropic glutamate receptor 8 expressing nerve terminals target subsets of GABAergic neurons in the hippocampus. J Neurosci 25:10520-10536.

Ferraguti F and Shigemoto R (2006) Metabotropic glutamate receptors. Cell Tissue Res 326:483-504

Ferré S, Karcz-Kubicha M, Hope BT, Popoli P, Burgueño J, Gutiérrez MA, Casadó V, Fuxe K, Goldberg SR, Lluis C, et al. (2002) Synergistic interaction between adenosine A2A and glutamate mGlu5 receptors: implications for striatal neuronal function. Proc Natl Acad Sci USA 99:11940-11945.

Ferré S, Popoli P, Rimondini R, Reggio R, Kehr J, and Fuxe K (1999) Adenosine A2A and group I metabotropic glutamate receptors synergistically modulate the binding characteristics of dopamine D2 receptors in the rat striatum. Neuropharmacology 38:129-140.

Fisher NM, Seto M, Lindsley CW, and Niswender CM (2018) Metabotropic glutamate receptor 7: a new therapeutic target in neurodevelopmental disorders. Front Mol Neurosci 11:387. 
Flajolet M, Rakhilin S, Wang H, Starkova N, Nuangchamnong N, Nairn AC, and Greengard P (2003) Protein phosphatase 2C binds selectively to and dephosphorylates metabotropic glutamate receptor 3. Proc Natl Acad Sci USA 100: $16006-16011$

Flavin HJ, Jin XT, and Daw NW (2000) 2R,4R-4-Aminopyrrolidine-2,4-dicarboxylate (APDC) attenuates cortical EPSPs. Brain Res 873:212-217.

Flor PJ, Lindauer K, Püttner I, Rüegg D, Lukic S, Knöpfel T, and Kuhn R (1995a) Molecular cloning, functional expression and pharmacological characterization of the human metabotropic glutamate receptor type 2. Eur J Neurosci 7 $622-629$.

Flor PJ, Lukic S, Rüegg D, Leonhardt T, Knöpfel T, and Kuhn R (1995b) Molecular cloning, functional expression and pharmacological characterization of the human metabotropic glutamate receptor type 4. Neuropharmacology 34:149-155.

Flor PJ, Van Der Putten H, Rüegg D, Lukic S, Leonhardt T, Bence M, Sansig G, Knöpfel T, and Kuhn R (1997) A novel splice variant of a metabotropic glutamate receptor, human mGluR7b. Neuropharmacology 36:153-159.

Font J, López-Cano M, Notartomaso S, Scarselli P, Di Pietro P, Bresolí-Obach R, Battaglia G, Malhaire F, Rovira X, Catena J, et al. (2017) Optical control of pain in vivo with a photoactive $\mathrm{mGlu}_{5}$ receptor negative allosteric modulator [published correction appears in eLife (2018) 7:e34752]. eLife 6:e23545.

Fotuhi M, Standaert DG, Testa CM, Penney JB Jr., and Young AB (1994) Differential expression of metabotropic glutamate receptors in the hippocampus and entorhinal cortex of the rat. Brain Res Mol Brain Res 21:283-292.

Francesconi A and Duvoisin RM (1998) Role of the second and third intracellular loops of metabotropic glutamate receptors in mediating dual signal transduction activation. J Biol Chem 273:5615-5624.

Francesconi A and Duvoisin RM (2000) Opposing effects of protein kinase C and protein kinase A on metabotropic glutamate receptor signaling: selective desensitization of the inositol trisphosphate/Ca2+ pathway by phosphorylation of the receptor-G protein-coupling domain. Proc Natl Acad Sci USA 97:6185-6190.

Francesconi A and Duvoisin RM (2002) Alternative splicing unmasks dendritic and axonal targeting signals in metabotropic glutamate receptor 1 . J Neurosci 22 $2196-2205$.

Francesconi A, Kumari R, and Zukin RS (2009a) Proteomic analysis reveals novel binding partners of metabotropic glutamate receptor $1 . J$ Neurochem 108: $1515-1525$

Francesconi A, Kumari R, and Zukin RS (2009b) Regulation of group I metabotropic glutamate receptor trafficking and signaling by the caveolar/lipid raft pathway. J Neurosci 29:3590-3602.

Frank RA, McRae AF, Pocklington AJ, van de Lagemaat LN, Navarro P, Croning MD, Komiyama NH, Bradley SJ, Challiss RA, Armstrong JD, et al. (2011) Clustered coding variants in the glutamate receptor complexes of individuals with schizophrenia and bipolar disorder. PLoS One 6:e19011.

Fribourg M, Moreno JL, Holloway T, Provasi D, Baki L, Mahajan R, Park G, Adney SK, Hatcher C, Eltit JM, et al. (2011) Decoding the signaling of a GPCR heteromeric complex reveals a unifying mechanism of action of antipsychotic drugs. Cell 147:1011-1023.

Fujinaga M, Yamasaki T, Kawamura K, Kumata K, Hatori A, Yui J, Yanamoto K, Yoshida Y, Ogawa M, Nengaki N, et al. (2011) Synthesis and evaluation of 6-[1-(2[(18)F]fluoro-3-pyridyl)-5-methyl-1H-1,2,3-triazol-4-yl]quinoline for positron emission tomography imaging of the metabotropic glutamate receptor type 1 in brain. Bioorg Med Chem 19:102-110.

Fukuda H, Tanaka T, Kaijima M, Nakai H, and Yonemasu Y (1985) Quisqualic acidinduced hippocampal seizures in unanesthetized cats. Neurosci Lett 59:53-59.

Fukuda J, Suzuki G, Kimura T, Nagatomi Y, Ito S, Kawamoto H, Ozaki S, and Ohta $\mathrm{H}$ (2009) Identification of a novel transmembrane domain involved in the negative modulation of mGluR1 using a newly discovered allosteric mGluR1 antagonist, 3cyclohexyl-5-fluoro-6-methyl-7-(2-morpholin-4-ylethoxy)-4H-chromen-4-one. Neuropharmacology 57:438-445.

Fukunaga I, Yeo CH, and Batchelor AM (2007) Potent and specific action of the mGlu1 antagonists YM-298198 and JNJ16259685 on synaptic transmission in rat cerebellar slices. Br J Pharmacol 151:870-876.

Fuzzati-Armentero MT, Cerri S, Levandis G, Ambrosi G, Montepeloso E, Antoninetti G, Blandini F, Baqi Y, Müller CE, Volpini R, et al. (2015) Dual target strategy: combining distinct non-dopaminergic treatments reduces neuronal cell loss and synergistically modulates L-DOPA-induced rotational behavior in a rodent model of Parkinson's disease. J Neurochem 134:740-747.

Gabra BH, Smith FL, Navarro HA, Carroll FI, and Dewey WL (2008) mGluR5 antagonists that block calcium mobilization in vitro also reverse (S)-3,5-DHPGinduced hyperalgesia and morphine antinociceptive tolerance in vivo. Brain Res 1187:58-66.

Galici R, Echemendia NG, Rodriguez AL, and Conn PJ (2005) A selective allosteric potentiator of metabotropic glutamate (mGlu) 2 receptors has effects similar to an orthosteric mGlu2/3 receptor agonist in mouse models predictive of antipsychotic activity. J Pharmacol Exp Ther 315:1181-1187.

Galici R, Jones CK, Hemstapat K, Nong Y, Echemendia NG, Williams LC, de Paulis T, and Conn PJ (2006) Biphenyl-indanone A, a positive allosteric modulator of the metabotropic glutamate receptor subtype 2 , has antipsychotic- and anxiolytic-like effects in mice. $J$ Pharmacol Exp Ther 318:173-185.

Gandhi RM, Kogan CS, and Messier C (2014) 2-Methyl-6-(phenylethynyl) pyridine (MPEP) reverses maze learning and PSD-95 deficits in Fmr1 knock-out mice. Front Cell Neurosci 8:70.

Gantois I, Pop AS, de Esch CE, Buijsen RA, Pooters T, Gomez-Mancilla B, Gasparin F, Oostra BA, D'Hooge R, and Willemsen R (2013) Chronic administration of AFQ056/Mavoglurant restores social behaviour in Fmr1 knockout mice. Behav Brain Res 239:72-79.

Garcia-Barrantes PM, Cho HP, Blobaum AL, Niswender CM, Conn PJ, and Lindsley CW (2015a) Lead optimization of the VU0486321 series of mGlu1 PAMs. Part 1 SAR of modifications to the central aryl core. Bioorg Med Chem Lett 25:5107-5110.

Garcia-Barrantes PM, Cho HP, Blobaum AL, Niswender CM, Conn PJ, and Lindsley CW (2016a) Lead optimization of the VU0486321 series of mGlu1 PAMs. Part 3.
Engineering plasma stability by discovery and optimization of isoindolinone analogs. Bioorg Med Chem Lett 26:1869-1872.

Garcia-Barrantes PM, Cho HP, Niswender CM, Byers FW, Locuson CW, Blobaum AL, Xiang Z, Rook JM, Conn PJ, and Lindsley CW (2015b) Development of novel, CNS penetrant positive allosteric modulators for the metabotropic glutamate receptor subtype 1 (mGlu1), based on an N-(3-Chloro-4-(1,3-dioxoisoindolin-2-yl) phenyl)-3-methylfuran-2-carboxamide scaffold, that potentiate wild type and mutant mGlu1 receptors found in schizophrenics. J Med Chem 58:7959-7971.

Garcia-Barrantes PM, Cho HP, Starr TM, Blobaum AL, Niswender CM, Conn PJ,

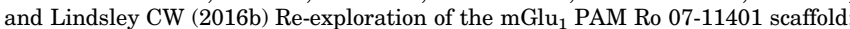
discovery of analogs with improved CNS penetration despite steep SAR. Bioorg Med Chem Lett 26:2289-2292.

García-Bea A, Bermudez I, Harrison PJ, and Lane TA (2017) A group II metabotropic glutamate receptor 3 (mGlu3, GRM3) isoform implicated in schizophrenia interacts with canonical mGlu3 and reduces ligand binding. J Psychopharmacol 31:1519-1526.

Gasparini F, Bruno V, Battaglia G, Lukic S, Leonhardt T, Inderbitzin W, Laurie D, Sommer B, Varney MA, Hess SD, et al. (1999a) (R,S)-4-phosphonophenylglycine, a potent and selective group III metabotropic glutamate receptor agonist, is anticonvulsive and neuroprotective in vivo. J Pharmacol Exp Ther 289:1678-1687.

Gasparini F, Lingenhöhl K, Stoehr N, Flor PJ, Heinrich M, Vranesic I, Biollaz M, Allgeier H, Heckendorn R, Urwyler S, et al. (1999b) 2-Methyl-6-(phenylethynyl)pyridine (MPEP), a potent, selective and systemically active mGlu5 receptor antagonist. Neuropharmacology 38:1493-1503.

Gass JT, McGonigal JT, and Chandler LJ (2017) Deficits in the extinction of ethanolseeking behavior following chronic intermittent ethanol exposure are attenuated with positive allosteric modulation of mGlu5. Neuropharmacology 113:198-205

Gass JT and Olive MF (2009) Role of protein kinase C epsilon (PKCvarepsilon) in the reduction of ethanol reinforcement due to mGluR5 antagonism in the nucleus accumbens shell. Psychopharmacology (Berl) 204:587-597.

Gass JT, Osborne MP, Watson NL, Brown JL, and Olive MF (2009) mGluR5 antagonism attenuates methamphetamine reinforcement and prevents reinstatement of methamphetamine-seeking behavior in rats. Neuropsychopharmacology 34:820-833.

Gastambide F, Cotel MC, Gilmour G, O'Neill MJ, Robbins TW, and Tricklebank MD (2012) Selective remediation of reversal learning deficits in the neurodevelopmental MAM model of schizophrenia by a novel mGlu5 positive allosteric modulator. Neuropsychopharmacology 37:1057-1066.

Gastambide F, Gilmour G, Robbins TW, and Tricklebank MD (2013) The mGlu positive allosteric modulator LSN2463359 differentially modulates motor, instrumental and cognitive effects of NMDA receptor antagonists in the rat. Neuropharmacology 64:240-247.

Gautier A, Juillerat A, Heinis C, Corrêa IR Jr., Kindermann M, Beaufils F, and Johnsson K (2008) An engineered protein tag for multiprotein labeling in living cells. Chem Biol 15:128-136.

Gawel K, Jenda-Wojtanowska M, Gibula-Bruzda E, Kedzierska E, Filarowska J, Marszalek-Grabska M, Wojtanowski KK, Komsta L, Talarek S, and Kotlinska JH (2018) The influence of AMN082, metabotropic glutamate receptor 7 (mGlu7) allosteric agonist on the acute and chronic antinociceptive effects of morphine in the tail-immersion test in mice: Comparison with mGlu5 and mGlu2/3 ligands. Physiol Behav 185:112-120.

Gee CE, Peterlik D, Neuhäuser C, Bouhelal R, Kaupmann K, Laue G, UscholdSchmidt N, Feuerbach D, Zimmermann K, Ofner S, et al. (2014) Blocking metabotropic glutamate receptor subtype 7 (mGlu7) via the Venus flytrap domain (VFTD) inhibits amygdala plasticity, stress, and anxiety-related behavior. J Biol Chem 289:10975-10987.

Gelb T, Pshenichkin S, Hathaway HA, Grajkowska E, Dalley CB, Wolfe BB, and Wroblewski JT (2015a) Atypical signaling of metabotropic glutamate receptor 1 in human melanoma cells. Biochem Pharmacol 98:182-189.

Gelb T, Pshenichkin S, Rodriguez OC, Hathaway HA, Grajkowska E, DiRaddo JO, Wroblewska B, Yasuda RP, Albanese C, Wolfe BB, et al. (2015b) Metabotropic glutamate receptor 1 acts as a dependence receptor creating a requirement for glutamate to sustain the viability and growth of human melanomas. Oncogene $\mathbf{3 4}$ $2711-2720$

Gereau RW IV and Conn PJ (1995) Multiple presynaptic metabotropic glutamate receptors modulate excitatory and inhibitory synaptic transmission in hippocampal area CA1. J Neurosci 15:6879-6889.

Gereau RW IV and Heinemann SF (1998) Role of protein kinase C phosphorylation in rapid desensitization of metabotropic glutamate receptor 5. Neuron 20:143-151.

Gerlai R, Adams B, Fitch T, Chaney S, and Baez M (2002) Performance deficits of mGluR8 knockout mice in learning tasks: the effects of null mutation and the background genotype. Neuropharmacology 43:235-249.

Gerlai R, Roder JC, and Hampson DR (1998) Altered spatial learning and memory in mice lacking the mGluR4 subtype of metabotropic glutamate receptor. Behav Neurosci 112:525-532.

Ghoshal A, Moran SP, Dickerson JW, Joffe ME, Grueter BA, Xiang Z, Lindsley CW, Rook JM, and Conn PJ (2017) Role of $\mathrm{mGlu}_{5}$ receptors and inhibitory neurotransmission in $\mathrm{M}_{1}$ dependent muscarinic LTD in the prefrontal cortex: implications in schizophrenia. ACS Chem Neurosci 8:2254-2265.

Gilmour G, Broad LM, Wafford KA, Britton T, Colvin EM, Fivush A, Gastambide F, Getman B, Heinz BA, McCarthy AP, et al. (2013) In vitro characterisation of the novel positive allosteric modulators of the mGlu $_{5}$ receptor, LSN2463359 and LSN2814617, and their effects on sleep architecture and operant responding in the rat. Neuropharmacology 64:224-239.

Girard B, Tuduri P, Moreno MP, Sakkaki S, Barboux C, Bouschet T, Varrault A, Vitre J, McCort-Tranchepain I, Dairou J, et al. (2019) The mGlu7 receptor provides protective effects against epileptogenesis and epileptic seizures. Neurobiol Dis 129 $13-28$

Goddyn H, Callaerts-Vegh Z, and D'Hooge R (2015) Functional dissociation of group III metabotropic glutamate receptors revealed by direct comparison between the behavioral profiles of knockout mouse lines. Int J Neuropsychopharmacol 18: pyv053. 
Goeldner C, Ballard TM, Knoflach F, Wichmann J, Gatti S, and Umbricht D (2013) Cognitive impairment in major depression and the mGlu2 receptor as a therapeutic target. Neuropharmacology 64:337-346.

Gogliotti RG, Senter RK, Fisher NM, Adams J, Zamorano R, Walker AG, Blobaum AL, Engers DW, Hopkins CR, Daniels JS, et al. (2017) $\mathrm{mGlu}_{7}$ potentiation rescues cognitive, social, and respiratory phenotypes in a mouse model of Rett syndrome. Sci Transl Med 9:eaai7459.

Golubeva AV, Moloney RD, O'Connor RM, Dinan TG, and Cryan JF (2016) Metabotropic glutamate receptors in central nervous system diseases. Curr Drug Targets 17:538-616.

Gómez-Santacana X, Pittolo S, Rovira X, Lopez M, Zussy C, Dalton JA, Faucherre A Jopling C, Pin JP, Ciruela F, et al. (2017) Illuminating phenylazopyridines to photoswitch metabotropic glutamate receptors: from the flask to the animals. ACS Cent Sci 3:81-91.

González-Maeso J, Ang RL, Yuen T, Chan P, Weisstaub NV, López-Giménez JF, Zhou M, Okawa Y, Callado LF, Milligan G, et al. (2008) Identification of a serotonin/glutamate receptor complex implicated in psychosis. Nature 452:93-97.

Goudet C, Chapuy E, Alloui A, Acher F, Pin JP, and Eschalier A (2008) Group III metabotropic glutamate receptors inhibit hyperalgesia in animal models of inflammation and neuropathic pain. Pain 137:112-124.

Goudet C, Kniazeff J, Hlavackova V, Malhaire F, Maurel D, Acher F, Blahos J, Prézeau L, and Pin JP (2005) Asymmetric functioning of dimeric metabotropic glutamate receptors disclosed by positive allosteric modulators. J Biol Chem $\mathbf{2 8 0}$ 24380-24385.

Goudet C, Rovira X, and Llebaria A (2018) Shedding light on metabotropic glutamate receptors using optogenetics and photopharmacology. Curr Opin Pharmacol 38: $8-15$

Goudet C, Vilar B, Courtiol T, Deltheil T, Bessiron T, Brabet I, Oueslati N, Rigault D, Bertrand HO, McLean H, et al. (2012) A novel selective metabotropic glutamate receptor 4 agonist reveals new possibilities for developing subtype selective ligands with therapeutic potential. FASEB J 26:1682-1693.

Gould RW, Amato RJ, Bubser M, Joffe ME, Nedelcovych MT, Thompson AD, Nickols HH, Yuh JP, Zhan X, Felts AS, et al. (2016) Partial mGlu 5 negative allosteric modulators attenuate cocaine-mediated behaviors and lack psychotomimetic-like effects. Neuropsychopharmacology 41:1166-1178.

Greco B, Invernizzi RW, and Carli M (2005) Phencyclidine-induced impairment in attention and response control depends on the background genotype of mice: reversal by the mGLU(2/3) receptor agonist LY379268. Psychopharmacology (Berl) 179:68-76.

Gregg LC, Jung KM, Spradley JM, Nyilas R, Suplita RL II, Zimmer A, Watanabe M, Mackie K, Katona I, Piomelli D, et al. (2012) Activation of type 5 metabotropic glutamate receptors and diacylglycerol lipase- $\alpha$ initiates 2-arachidonoylglycerol formation and endocannabinoid-mediated analgesia. J Neurosci 32:9457-9468.

Gregory KJ, Bridges TM, Gogliotti RG, Stauffer SR, Noetzel MJ, Jones CK, Lindsley CW, Conn PJ, and Niswender CM (2019) In vitro to in vivo translation of allosteric modulator concentration-effect relationships: implications for drug discovery. ACS Pharmacol Transl Sci 2:442-452.

Gregory KJ, Giraldo J, Diao J, Christopoulos A, and Leach K (2020) Evaluation of operational models of agonism and allosterism at receptors with multiple orthosteric binding sites. Mol Pharmacol 97:35-45.

Gregory KJ, Herman EJ, Ramsey AJ, Hammond AS, Byun NE, Stauffer SR, Manka JT, Jadhav S, Bridges TM, Weaver CD, et al. (2013a) N-aryl piperazine metabotropic glutamate receptor 5 positive allosteric modulators possess efficacy in preclinical models of NMDA hypofunction and cognitive enhancement. $J$ Pharmacol Exp Ther 347:438-457.

Gregory KJ, Kufareva I, Keller AN, Khajehali E, Mun H-C, Goolam MA, Mason RS, Capuano B, Conigrave AD, Christopoulos A, et al. (2018) Dual action calciumsensing receptor modulator unmasks novel mode-switching mechanism. ACS Pharmacol Transl Sci 1:96-109.

Gregory KJ, Malosh C, Turlington M, Morrison R, Vinson P, Daniels JS, Jones C, Niswender CM, Conn PJ, Lindsley CW, et al. (2010a) Identification of a high affinity MPEP-site silent allosteric modulator (SAM) for the metabotropic glutamate subtype 5 receptor (mGlu5), Probe Reports from the NIH Molecular Libraries Program, National Center for Biotechnology Information (US), Bethesda, MD.

Gregory KJ, Nguyen ED, Malosh C, Mendenhall JL, Zic JZ, Bates BS, Noetzel MJ, Squire EF, Turner EM, Rook JM, et al. (2014) Identification of specific ligandreceptor interactions that govern binding and cooperativity of diverse modulators to a common metabotropic glutamate receptor 5 allosteric site. ACS Chem Neurosci 5:282-295.

Gregory KJ, Nguyen ED, Reiff SD, Squire EF, Stauffer SR, Lindsley CW, Meiler J, and Conn PJ (2013b) Probing the metabotropic glutamate receptor $5\left(\mathrm{mGlu}_{5}\right)$ positive allosteric modulator (PAM) binding pocket: discovery of point mutations that engender a "molecular switch" in PAM pharmacology. Mol Pharmacol 83 991-1006.

Gregory KJ, Noetzel MJ, Rook JM, Vinson PN, Stauffer SR, Rodriguez AL, Emmitte KA, Zhou Y, Chun AC, Felts AS, et al. (2012) Investigating metabotropic glutamate receptor 5 allosteric modulator cooperativity, affinity, and agonism: enriching structurefunction studies and structure-activity relationships. Mol Pharmacol 82:860-875.

Gregory KJ, Sexton PM, and Christopoulos A (2010b) Overview of receptor allosterism. Curr Protoc Pharmacol Chapter 1:Unit 1.21.

Gregory KJ, Velagaleti R, Thal DM, Brady RM, Christopoulos A, Conn PJ, and Lapinsky DJ (2016) Clickable photoaffinity ligands for metabotropic glutamate receptor 5 based on select acetylenic negative allosteric modulators. ACS Chem Biol 11:1870-1879.

Griebel G, Pichat P, Boulay D, Naimoli V, Potestio L, Featherstone R, Sahni S, Defex H, Desvignes C, Slowinski F, et al. (2016) The mGluR2 positive allosteric modulator, SAR218645, improves memory and attention deficits in translational models of cognitive symptoms associated with schizophrenia. Sci Rep 6:35320.

Grillon C, Cordova J, Levine LR, and Morgan CA III (2003) Anxiolytic effects of a novel group II metabotropic glutamate receptor agonist (LY354740) in the fearpotentiated startle paradigm in humans. Psychopharmacology (Berl) 168:446-454.
Gulia R, Sharma R, and Bhattacharyya S (2017) A critical role for ubiquitination in the endocytosis of glutamate receptors. J Biol Chem 292:1426-1437.

Gutzeit VA, Thibado J, Stor DS, Zhou Z, Blanchard SC, Andersen OS, and Levitz J (2019) Conformational dynamics between transmembrane domains and allosteric modulation of a metabotropic glutamate receptor. eLife 8:e45116.

Haas HS, Pfragner R, Siegl V, Ingolic E, Heintz E, Schraml E, and Schauenstein K (2007) The non-competitive metabotropic glutamate receptor-1 antagonist CPCCOEt inhibits the in vitro growth of human melanoma. Oncol Rep 17: 1399-1404

Haas LT, Salazar SV, Smith LM, Zhao HR, Cox TO, Herber CS, Degnan AP, Balakrishnan A, Macor JE, Albright CF, et al (2017) Silent allosteric modulation of mGluR5 maintains glutamate signaling while rescuing Alzheimer's mouse phenotypes. Cell Rep 20:76-88.

Habrian CH, Levitz J, Vyklicky V, Fu Z, Hoagland A, McCort-Tranchepain I, Acher F, and Isacoff EY (2019) Conformational pathway provides unique sensitivity to a synaptic mGluR. Nat Commun 10:5572

Hackler EA, Byun NE, Jones CK, Williams JM, Baheza R, Sengupta S, Grier MD, Avison M, Conn PJ, and Gore JC (2010) Selective potentiation of the metabotropic glutamate receptor subtype 2 blocks phencyclidine-induced hyperlocomotion and brain activation. Neuroscience 168:209-218.

Hajasova Z, Canestrelli C, Acher F, Noble F, and Marie N (2018) Role of mGlu7 receptor in morphine rewarding effects is uncovered by a novel orthosteric agonist. Neuropharmacology 131:424-430.

Halberstadt AL, van der Zee JVF, Chatha M, Geyer MA, and Powell SB (2019) Chronic treatment with a metabotropic mGlu2/3 receptor agonist diminishes behavioral response to a phenethylamine hallucinogen. Psychopharmacology (Berl) 236:821-830.

Hall DA (2013) Application of receptor theory to allosteric modulation of receptors. Prog Mol Biol Transl Sci 115:217-290.

Hall DA and Giraldo J (2018) A method for the quantification of biased signalling at constitutively active receptors. Br J Pharmacol 175:2046-2062.

Hamilton A, Vasefi M, Vander Tuin C, McQuaid RJ, Anisman H, and Ferguson SS (2016) Chronic pharmacological mGluR5 inhibition prevents cognitive impairment and reduces pathogenesis in an Alzheimer disease mouse model. Cell Rep $\mathbf{1 5}$ $1859-1865$

Hammond AS, Rodriguez AL, Townsend SD, Niswender CM, Gregory KJ, Lindsley CW, and Conn PJ (2010) Discovery of a novel chemical class of mGlu(5) allosteric ligands with distinct modes of pharmacology. ACS Chem Neurosci 1:702-716.

Hanak TJ, Libbey JE, Doty DJ, Sim JT, DePaula-Silva AB, and Fujinami RS (2019) Positive modulation of mGluR5 attenuates seizures and reduces TNF- $\alpha^{+}$macrophages and microglia in the brain in a murine model of virus-induced temporal lobe epilepsy. Exp Neurol 311:194-204.

Hanna L, Ceolin L, Lucas S, Monn J, Johnson B, Collingridge G, Bortolotto Z, and Lodge D (2013) Differentiating the roles of mGlu2 and mGlu3 receptors using LY541850, an mGlu2 agonist/mGlu3 antagonist. Neuropharmacology 66:114-121. Harich S, Gross G, and Bespalov A (2007) Stimulation of the metabotropic glutamate $2 / 3$ receptor attenuates social novelty discrimination deficits induced by neonata phencyclidine treatment. Psychopharmacology (Berl) 192:511-519.

Harvey BD, Siok CJ, Kiss T, Volfson D, Grimwood S, Shaffer CL, and Hajós M (2013) Neurophysiological signals as potential translatable biomarkers for modulation of metabotropic glutamate 5 receptors. Neuropharmacology 75:19-30.

Harvey J and Collingridge GL (1993) Signal transduction pathways involved in the acute potentiation of NMDA responses by $1 \mathrm{~S}, 3 \mathrm{R}-\mathrm{ACPD}$ in rat hippocampal slices. Br J Pharmacol 109:1085-1090.

Hathaway HA, Pshenichkin S, Grajkowska E, Gelb T, Emery AC, Wolfe BB, and Wroblewski JT (2015) Pharmacological characterization of mGlu1 receptors in cerebellar granule cells reveals biased agonism. Neuropharmacology 93:199-208.

Hayashi Y, Momiyama A, Takahashi T, Ohishi H, Ogawa-Meguro R, Shigemoto R, Mizuno N, and Nakanishi S (1993) Role of a metabotropic glutamate receptor in synaptic modulation in the accessory olfactory bulb. Nature 366:687-690.

Hellyer SD, Aggarwal S, Chen ANY, Leach K, Lapinsky DJ, and Gregory KJ (2020) Development of clickable photoaffinity ligands for metabotropic glutamate receptor 2 based on two positive allosteric modulator chemotypes. ACS Chem Neurosci 11 1597-1609.

Hellyer SD, Albold S, Sengmany K, Singh J, Leach K, and Gregory KJ (2019) Metabotropic glutamate receptor $5\left(\mathrm{mGlu}_{5}\right)$-positive allosteric modulators differentially induce or potentiate desensitization of $\mathrm{mGlu}_{5}$ signaling in recombinant cells and neurons. J Neurochem 151:301-315

Hellyer SD, Albold S, Wang T, Chen ANY, May LT, Leach K, and Gregory KJ (2018) "Selective" class C G protein-coupled receptor modulators are neutral or biased $\mathrm{mGlu}_{5}$ allosteric ligands. Mol Pharmacol 93:504-514.

Hellyer SD, Sengmany K, Keller AN, Christopoulos A, Leach K, and Gregory KJ (2020) Probe dependence and biased potentiation of metabotropic glutamate receptor 5 is mediated by differential ligand interactions in the common allosteric binding site. Biochem Pharmacol 177:114013.

Helton DR, Tizzano JP, Monn JA, Schoepp DD, and Kallman MJ (1997) LY354740: a metabotropic glutamate receptor agonist which ameliorates symptoms of nicotine withdrawal in rats. Neuropharmacology 36:1511-1516.

Helton DR, Tizzano JP, Monn JA, Schoepp DD, and Kallman MJ (1998) Anxiolytic and side-effect profile of LY354740: a potent, highly selective, orally active agonist for group II metabotropic glutamate receptors. J Pharmacol Exp Ther 284: 651-660.

Hemstapat K, Da Costa H, Nong Y, Brady AE, Luo Q, Niswender CM, Tamagnan GD, and Conn PJ (2007) A novel family of potent negative allosteric modulators of group II metabotropic glutamate receptors. J Pharmacol Exp Ther 322:254-264.

Hemstapat K, de Paulis T, Chen Y, Brady AE, Grover VK, Alagille D, Tamagnan GD, and Conn PJ (2006) A novel class of positive allosteric modulators of metabotropic glutamate receptor subtype 1 interact with a site distinct from that of negative allosteric modulators. Mol Pharmacol 70:616-626.

Henrich-Noack P, Hatton CD, and Reymann KG (1998) The mGlu receptor ligand (S) $4 \mathrm{C} 3 \mathrm{HPG}$ protects neurons after global ischaemia in gerbils. Neuroreport 9:985-988. 
Henrich-Noack P and Reymann KG (1999) (1S,3R)-ACPD, a metabotropic glutamate receptor agonist, enhances damage after global ischaemia. Eur J Pharmacol 365:55-58.

Hermans E, Nahorski SR, and Challiss RA (1998) Reversible and non-competitive antagonist profile of CPCCOEt at the human type 1alpha metabotropic glutamate receptor. Neuropharmacology 37:1645-1647.

Hikichi H, Kaku A, Karasawa J, and Chaki S (2013) Stimulation of metabotropic glutamate $(\mathrm{mGlu}) 2$ receptor and blockade of mGlu1 receptor improve social memory impairment elicited by MK-801 in rats. J Pharmacol Sci 122:10-16.

Hikichi H, Murai T, Okuda S, Maehara S, Satow A, Ise S, Nishino M, Suzuki G, Takehana H, Hata M, et al. (2010a) Effects of a novel metabotropic glutamate receptor 7 negative allosteric modulator, 6-(4-methoxyphenyl)-5-methyl-3-pyridin4-ylisoxazonolo[4,5-c]pyridin-4(5H)-one (MMPIP), on the central nervous system in rodents. Eur J Pharmacol 639:106-114.

Hikichi H, Nishino M, Fukushima M, Satow A, Maehara S, Kawamoto H, and Ohta H (2010b) Pharmacological effects of metabotropic glutamate receptor ligands on prepulse inhibition in DBA/2J mice. Eur J Pharmacol 639:99-105.

Hinoi E, Ogita K, Takeuchi Y, Ohashi H, Maruyama T, and Yoneda Y (2000) Direct radiolabeling by $[3 \mathrm{H}]$ quisqualic acid of group I metabotropic glutamate receptor in rat brain synaptic membranes. Brain Res 881:199-203.

Hirbec H, Perestenko O, Nishimune A, Meyer G, Nakanishi S, Henley JM, and Dev KK (2002) The PDZ proteins PICK1, GRIP, and syntenin bind multiple glutamate receptor subtypes. Analysis of PDZ binding motifs. J Biol Chem 277:15221-15224.

Hiyoshi T, Marumo T, Hikichi H, Tomishima Y, Urabe H, Tamita T, Iida I, Yasuhara A, Karasawa J, and Chaki S (2014) Neurophysiologic and antipsychotic profiles of TASP0433864, a novel positive allosteric modulator of metabotropic glutamate 2 receptor. J Pharmacol Exp Ther 351:642-653

Hlavackova V, Goudet C, Kniazeff J, Zikova A, Maurel D, Vol C, Trojanova J, Prézeau L, Pin JP, and Blahos J (2005) Evidence for a single heptahelical domain being turned on upon activation of a dimeric GPCR. EMBO J 24:499-509.

Hodge CW, Miles MF, Sharko AC, Stevenson RA, Hillmann JR, Lepoutre V, Besheer J, and Schroeder JP (2006) The mGluR5 antagonist MPEP selectively inhibits the onset and maintenance of ethanol self-administration in C57BL/6J mice. Psychopharmacology (Berl) 183:429-438.

Hoffmann HM, Crouzin N, Moreno E, Raivio N, Fuentes S, McCormick PJ, Ortiz J, and Vignes M (2017) Long-lasting impairment of mGluR5-activated intracellular pathways in the striatum after withdrawal of cocaine self-administration. Int $J$ Neuropsychopharmacol 20:72-82.

Holmes GL, Thurber SJ, Liu Z, Stafstrom CE, Gatt A, and Mikati MA (1993) Effects of quisqualic acid and glutamate on subsequent learning, emotionality, and seizure susceptibility in the immature and mature animal. Brain Res 623:325-328.

Hong J, Lee J, Song K, Ha GE, Yang YR, Ma JS, Yamamoto M, Shin HS, Suh PG, and Cheong E (2016) The thalamic mGluR1-PLC $\beta 4$ pathway is critical in sleep architecture. Mol Brain 9:100.

Horio M, Fujita Y, and Hashimoto K (2013) Therapeutic effects of metabotropic glutamate receptor 5 positive allosteric modulator CDPPB on phencyclidineinduced cognitive deficits in mice. Fundam Clin Pharmacol 27:483-488.

Hou L and Klann E (2004) Activation of the phosphoinositide 3-kinase-Akt-mammalian target of rapamycin signaling pathway is required for metabotropic glutamate receptor-dependent long-term depression. J Neurosci 24:6352-6361.

Hsieh M-H, Ho S-C, Yeh K-Y, Pawlak CR, Chang H-M, Ho Y-J, Lai T-J, and Wu F-Y (2012) Blockade of metabotropic glutamate receptors inhibits cognition and neurodegeneration in an MPTP-induced Parkinson's disease rat model. Pharmacol Biochem Behav 102:64-71.

Hu JH, Yang L, Kammermeier PJ, Moore CG, Brakeman PR, Tu J, Yu S, Petralia RS, Li Z, Zhang PW, et al. (2012) Preso1 dynamically regulates group I metabotropic glutamate receptors. Nat Neurosci 15:836-844.

Hu YJ, Sun Q, Zhang WH, Huo YJ, Xu CJ, and Liu JF (2019) Specific activation of mGlu2 induced IGF-1R transactivation in vitro through FAK phosphorylation. Acta Pharmacol Sin 40:460-467.

Huang CC, Yang PC, Lin HJ, and Hsu KS (2007) Repeated cocaine administration impairs group II metabotropic glutamate receptor-mediated long-term depression in rat medial prefrontal cortex. $J$ Neurosci 27:2958-2968.

Huang Y, Shu H, Li L, Zhen T, Zhao J, Zhou X, and Luo W (2018) L-DOPA-induced motor impairment and overexpression of corticostriatal synaptic components are improved by the mGluR5 antagonist MPEP in 6-OHDA-lesioned rats. ASN Neuro 10: 1759091418811021.

Hughes ZA, Neal SJ, Smith DL, Sukoff Rizzo SJ, Pulicicchio CM, Lotarski S, Lu S, Dwyer JM, Brennan J, Olsen M, et al. (2013) Negative allosteric modulation of metabotropic glutamate receptor 5 results in broad spectrum activity relevant to treatment resistant depression. Neuropharmacology 66:202-214.

Hullinger R, O'Riordan K, and Burger C (2015) Environmental enrichment improves learning and memory and long-term potentiation in young adult rats through a mechanism requiring mGluR5 signaling and sustained activation of p70s6k Neurobiol Learn Mem 125:126-134.

Iacovelli L, Arcella A, Battaglia G, Pazzaglia S, Aronica E, Spinsanti P, Caruso A, De Smaele E, Saran A, Gulino A, et al. (2006) Pharmacological activation of mGlu4 metabotropic glutamate receptors inhibits the growth of medulloblastomas. J Neurosci 26:8388-8397.

Iacovelli L, Bruno V, Salvatore L, Melchiorri D, Gradini R, Caricasole A, Barletta E, De Blasi A, and Nicoletti F (2002) Native group-III metabotropic glutamate receptors are coupled to the mitogen-activated protein kinase/phosphatidylinositol3-kinase pathways. $J$ Neurochem 82:216-223.

Iacovelli L, Molinaro G, Battaglia G, Motolese M, Di Menna L, Alfiero M, Blahos J, Matrisciano F, Corsi M, Corti C, et al. (2009) Regulation of group II metabotropic glutamate receptors by $\mathrm{G}$ protein-coupled receptor kinases: mGlu2 receptors are resistant to homologous desensitization. Mol Pharmacol 75:991-1003.

Iacovelli L, Salvatore L, Capobianco L, Picascia A, Barletta E, Storto M, Mariggiò S, Sallese M, Porcellini A, Nicoletti F, et al. (2003) Role of G protein-coupled receptor kinase 4 and beta-arrestin 1 in agonist-stimulated metabotropic glutamate receptor 1 internalization and activation of mitogen-activated protein kinases. J Biol Chem 278:12433-12442.
Iderberg H, Maslava N, Thompson AD, Bubser M, Niswender CM, Hopkins CR, Lindsley CW, Conn PJ, Jones CK, and Cenci MA (2015) Pharmacological stimulation of metabotropic glutamate receptor type 4 in a rat model of Parkinson's disease and L-DOPA-induced dyskinesia: comparison between a positive allosteric modulator and an orthosteric agonist. Neuropharmacology $\mathbf{9 5}$ 121-129.

Isherwood SN, Robbins TW, Nicholson JR, Dalley JW, and Pekcec A (2017) Selective and interactive effects of $D_{2}$ receptor antagonism and positive allosteric mGluR4 modulation on waiting impulsivity. Neuropharmacology 123:249-260.

Ishikawa K, Nash SR, Nishimune A, Neki A, Kaneko S, and Nakanishi S (1999) Competitive interaction of seven in absentia homolog-1A and $\mathrm{Ca} 2+/$ calmodulin with the cytoplasmic tail of group 1 metabotropic glutamate receptors. Genes Cells 4:381-390.

Itil T, Seaman B, Huque M, Mukhopadhyay S, Blasucci D, Nq K, and Ciccone P (1978) The clinical and quantitative EEG effects and plasma levels of fenobam (McN-3377) in subjects with anxiety: an open rising dose tolerance and efficacy study. Curr Ther Res Clin Exp 24:708-724.

Ito S, Hirata Y, Nagatomi Y, Satoh A, Suzuki G, Kimura T, Satow A, Maehara S, Hikichi H, Hata M, et al. (2009) Discovery and biological profile of isoindolinone derivatives as novel metabotropic glutamate receptor 1 antagonists: a potential treatment for psychotic disorders. Bioorg Med Chem Lett 19:5310-5313.

Jacob W, Gravius A, Pietraszek M, Nagel J, Belozertseva I, Shekunova E, Malyshkin A, Greco S, Barberi C, and Danysz W (2009) The anxiolytic and analgesic properties of fenobam, a potent mGlu5 receptor antagonist, in relation to the impairment of learning. Neuropharmacology 57:97-108.

Jacquemont S, Curie A, des Portes V, Torrioli MG, Berry-Kravis E, Hagerman RJ, Ramos FJ, Cornish K, He Y, Paulding C, et al. (2011) Epigenetic modification of the FMR1 gene in fragile $\mathrm{X}$ syndrome is associated with differential response to the mGluR5 antagonist AFQ056. Sci Transl Med 3:64ra1.

Jalan-Sakrikar N, Field JR, Klar R, Mattmann ME, Gregory KJ, Zamorano R, Engers DW, Bollinger SR, Weaver CD, Days EL, et al. (2014) Identification of positive allosteric modulators VU0155094 (ML397) and VU0422288 (ML396) reveals new insights into the biology of metabotropic glutamate receptor 7. ACS Chem Neurosci 5:1221-1237.

Jenda M, Gawel K, Marszalek M, Komsta L, and Kotlinska JH (2015) AMN082, a metabotropic glutamate receptor 7 allosteric agonist, attenuates locomotor sensitization and cross-sensitization induced by cocaine and morphine in mice. Prog Neuropsychopharmacol Biol Psychiatry 57:166-175.

Jia Z, Lu Y, Henderson J, Taverna F, Romano C, Abramow-Newerly W, Wojtowicz JM, and Roder J (1998) Selective abolition of the NMDA component of long-term potentiation in mice lacking mGluR5. Learn Mem 5:331-343.

Jiang JY, Nagaraju M, Meyer RC, Zhang L, Hamelberg D, Hall RA, Brown EM, Conn PJ, and Yang JJ (2014) Extracellular calcium modulates actions of orthosteric and allosteric ligands on metabotropic glutamate receptor 1 $\alpha$. J Biol Chem 289:1649-1661.

Jin DZ, Guo ML, Xue B, Fibuch EE, Choe ES, Mao LM, and Wang JQ (2013a) Phosphorylation and feedback regulation of metabotropic glutamate receptor 1 by calcium/calmodulin-dependent protein kinase II. J Neurosci 33:3402-3412.

Jin DZ, Guo ML, Xue B, Mao LM, and Wang JQ (2013b) Differential regulation of CaMKII $\alpha$ interactions with mGluR5 and NMDA receptors by $\mathrm{Ca}(2+)$ in neurons. $J$ Neurochem 127:620-631.

Jin DZ, Mao LM, and Wang JQ (2017) An essential role of Fyn in the modulation of metabotropic glutamate receptor 1 in neurons. eNeuro 4-ENEURO 0096-17.2017. Jin DZ, Mao LM, and Wang JQ (2018) The role of extracellular signal-regulated kinases (ERK) in the regulation of mGlu5 receptors in neurons. J Mol Neurosci 66:629-638.

Jin DZ, Xue B, Mao LM, and Wang JQ (2015) Metabotropic glutamate receptor 5 upregulates surface NMDA receptor expression in striatal neurons via CaMKII. Brain Res 1624:414-423.

Jin X, Semenova S, Yang L, Ardecky R, Sheffler DJ, Dahl R, Conn PJ, Cosford ND, and Markou A (2010) The mGluR2 positive allosteric modulator BINA decreases cocaine self-administration and cue-induced cocaine-seeking and counteracts cocaine-induced enhancement of brain reward function in rats. Neuropsychopharmacology 35:2021-2036.

Joffe ME, Santiago CI, Stansley BJ, Maksymetz J, Gogliotti RG, Engers JL, Nicoletti F, Lindsley CW, and Conn PJ (2019) Mechanisms underlying prelimbic prefronta cortex $\mathrm{mGlu}_{3} / \mathrm{mGlu}_{5}$-dependent plasticity and reversal learning deficits following acute stress. Neuropharmacology 144:19-28.

Johnson KA, Mateo Y, and Lovinger DM (2017) Metabotropic glutamate receptor 2 inhibits thalamically-driven glutamate and dopamine release in the dorsal striatum. Neuropharmacology 117:114-123.

Johnson MP, Baez M, Jagdmann GE Jr., Britton TC, Large TH, Callagaro DO, Tizzano JP, Monn JA, and Schoepp DD (2003) Discovery of allosteric potentiators for the metabotropic glutamate 2 receptor: synthesis and subtype selectivity of $\mathrm{N}$ (4-(2-methoxyphenoxy)phenyl)-N-(2,2,2- trifluoroethylsulfonyl)pyrid-3-ylmethylamine. J Med Chem 46:3189-3192.

Johnson MP, Barda D, Britton TC, Emkey R, Hornback WJ, Jagdmann GE, McKinzie DL, Nisenbaum ES, Tizzano JP, and Schoepp DD (2005) Metabotropic glutamate 2 receptor potentiators: receptor modulation, frequency-dependent synaptic activity, and efficacy in preclinical anxiety and psychosis model(s). Psychopharmacology (Berl) 179:271-283.

Johnson PL, Fitz SD, Engleman EA, Svensson KA, Schkeryantz JM, and Shekhar A (2013) Group II metabotropic glutamate receptor type 2 allosteric potentiators prevent sodium lactate-induced panic-like response in panic-vulnerable rats. J Psychopharmacol 27:152-161.

Joly C, Gomeza J, Brabet I, Curry K, Bockaert J, and Pin JP (1995) Molecular, functional, and pharmacological characterization of the metabotropic glutamate receptor type 5 splice variants: comparison with mGluR1. J Neurosci 15:3970-3981.

Jones CK, Bubser M, Thompson AD, Dickerson JW, Turle-Lorenzo N, Amalric M, Blobaum AL, Bridges TM, Morrison RD, Jadhav S, et al. (2012) The metabotropic glutamate receptor 4-positive allosteric modulator VU0364770 produces efficacy alone and in combination with L-DOPA or an adenosine 2A antagonist in preclinical rodent models of Parkinson's disease. J Pharmacol Exp Ther 340:404-421. 
Jones CK, Eberle EL, Peters SC, Monn JA, and Shannon HE (2005) Analgesic effects of the selective group II (mGlu2/3) metabotropic glutamate receptor agonists LY379268 and LY389795 in persistent and inflammatory pain models after acute and repeated dosing. Neuropharmacology 49 (Suppl 1):206-218.

Jong YI, Harmon SK, and O'Malley KL (2019) Location and cell-type-specific bias of metabotropic glutamate receptor, $\mathrm{mGlu}_{5}$, negative allosteric modulators. ACS Chem Neurosci 10:4558-4570.

Jong YJ, Kumar V, Kingston AE, Romano C, and O'Malley KL (2005) Functional metabotropic glutamate receptors on nuclei from brain and primary cultured striatal neurons. Role of transporters in delivering ligand. $J$ Biol Chem 280: 30469-30480.

Jong YJ, Kumar V, and O'Malley KL (2009) Intracellular metabotropic glutamate receptor 5 (mGluR5) activates signaling cascades distinct from cell surface counterparts. J Biol Chem 284:35827-35838.

Julio-Pieper M, Flor PJ, Dinan TG, and Cryan JF (2011) Exciting times beyond the brain: metabotropic glutamate receptors in peripheral and non-neural tissues. Pharmacol Rev 63:35-58.

Jung KM, Mangieri R, Stapleton C, Kim J, Fegley D, Wallace M, Mackie K, and Piomelli D (2005) Stimulation of endocannabinoid formation in brain slice cultures through activation of group I metabotropic glutamate receptors. $\mathrm{Mol}$ Pharmacol 68:1196-1202.

Justinova Z, Panlilio LV, Secci ME, Redhi GH, Schindler CW, Cross AJ, Mrzljak L, Medd A, Shaham Y, and Goldberg SR (2015) The novel metabotropic glutamate receptor 2 positive allosteric modulator, AZD8529, decreases nicotine selfsdministration and relapse in squirrel monkeys. Biol Psychiatry 78:452-462.

Kahl E and Fendt M (2016) Metabotropic glutamate receptors 7 within the nucleus accumbens are involved in relief learning in rats. Curr Neuropharmacol 14:405-412.

Kalinichev M, Le Poul E, Boléa C, Girard F, Campo B, Fonsi M, Royer-Urios I, Browne SE, Uslaner JM, Davis MJ, et al. (2014) Characterization of the novel positive allosteric modulator of the metabotropic glutamate receptor 4 ADX88178 in rodent models of neuropsychiatric disorders. J Pharmacol Exp Ther 350: 495-505

Kalinichev M, Rouillier M, Girard F, Royer-Urios I, Bournique B, Finn T, Charvin D, Campo B, Le Poul E, Mutel V, et al. (2013) ADX71743, a potent and selective negative allosteric modulator of metabotropic glutamate receptor 7: in vitro and in vivo characterization. J Pharmacol Exp Ther 344:624-636.

Kamiya H and Yamamoto C (1997) Phorbol ester and forskolin suppress the presynaptic inhibitory action of group-II metabotropic glutamate receptor at rat hippocampal mossy fibre synapse. Neuroscience 80:89-94.

Kammermeier PJ (2012) Functional and pharmacological characteristics of metabotropic glutamate receptors 2/4 heterodimers. Mol Pharmacol 82:438-447.

Kanumilli S, Toms NJ, Venkateswarlu K, Mellor H, and Roberts PJ (2002) Functional coupling of rat metabotropic glutamate 1a receptors to phospholipase $\mathrm{D}$ in $\mathrm{CHO}$ cells: involvement of extracellular $\mathrm{Ca} 2+$, protein kinase $\mathrm{C}$, tyrosine kinase and Rho-A. Neuropharmacology 42:1-8.

Kawabata S, Tsutsumi R, Kohara A, Yamaguchi T, Nakanishi S, and Okada M (1996) Control of calcium oscillations by phosphorylation of metabotropic glutamate receptors. Nature 383:89-92.

Kawaura K, Karasawa J, and Hikichi H (2016) Stimulation of the metabotropic glutamate (mGlu) 2 receptor attenuates the MK-801-induced increase in the immobility time in the forced swimming test in rats. Pharmacol Rep 68:80-84.

Keck TM, Yang HJ, Bi GH, Huang Y, Zhang HY, Srivastava R, Gardner EL, Newman $\mathrm{AH}$, and Xi ZX (2013) Fenobam sulfate inhibits cocaine-taking and cocaine-seeking behavior in rats: implications for addiction treatment in humans. Psychopharmacology (Berl) 229:253-265.

Kenakin T, Watson C, Muniz-Medina V, Christopoulos A, and Novick S (2012) A simple method for quantifying functional selectivity and agonist bias. ACS Chem Neurosci 3:193-203.

Kent JM, Daly E, Kezic I, Lane R, Lim P, De Smedt H, De Boer P, Van Nueten L, Drevets WC, and Ceusters M (2016) Efficacy and safety of an adjunctive mGlu2 receptor positive allosteric modulator to a SSRI/SNRI in anxious depression. Prog Neuropsychopharmacol Biol Psychiatry 67:66-73.

Keppler A, Gendreizig S, Gronemeyer T, Pick H, Vogel H, and Johnsson K (2003) A general method for the covalent labeling of fusion proteins with small molecules in vivo. Nat Biotechnol 21:86-89.

Keywood C, Wakefield M, and Tack J (2009) A proof-of-concept study evaluating the effect of ADX10059, a metabotropic glutamate receptor-5 negative allosteric modulator, on acid exposure and symptoms in gastro-oesophageal reflux disease. Gut 58:1192-1199.

Kim CH, Braud S, Isaac JT, and Roche KW (2005) Protein kinase C phosphorylation of the metabotropic glutamate receptor mGluR5 on Serine 839 regulates Ca2+ oscillations. J Biol Chem 280:25409-25415.

Kim J, Lee S, Park H, Song B, Hong I, Geum D, Shin K, and Choi S (2007) Blockade of amygdala metabotropic glutamate receptor subtype 1 impairs fear extinction. Biochem Biophys Res Commun 355:188-193.

Kim JH and Vezina P (1998) The metabotropic glutamate receptor antagonist (RS)MCPG produces hyperlocomotion in amphetamine pre-exposed rats. Neurophar macology 37:189-197.

Kim SH, Steele JW, Lee SW, Clemenson GD, Carter TA, Treuner K, Gadient R, Wedel P, Glabe C, Barlow C, et al. (2014) Proneurogenic Group II mGluR antagonist improves learning and reduces anxiety in Alzheimer $\mathrm{A} \beta$ oligomer mouse. Mol Psychiatry 19:1235-1242.

Kingston AE, O’Neill MJ, Lam A, Bales KR, Monn JA, and Schoepp DD (1999) Neuroprotection by metabotropic glutamate receptor glutamate receptor agonists: LY354740, LY379268 and LY389795. Eur J Pharmacol 377:155-165.

Kingston AE, Ornstein PL, Wright RA, Johnson BG, Mayne NG, Burnett JP, Belagaje R, Wu S, and Schoepp DD (1998) LY341495 is a nanomolar potent and selective antagonis of group II metabotropic glutamate receptors. Neuropharmacology 37:1-12.

Kinney GA and Slater NT (1993) Potentiation of NMDA receptor-mediated transmission in turtle cerebellar granule cells by activation of metabotropic glutamate receptors. J Neurophysiol 69:585-594.
Kinney GG, Burno M, Campbell UC, Hernandez LM, Rodriguez D, Bristow LJ, and Conn PJ (2003) Metabotropic glutamate subtype 5 receptors modulate locomotor activity and sensorimotor gating in rodents. J Pharmacol Exp Ther 306: 116-123.

Kinney GG, O’Brien JA, Lemaire W, Burno M, Bickel DJ, Clements MK, Chen TB, Wisnoski DD, Lindsley CW, Tiller PR, et al. (2005) A novel selective positive allosteric modulator of metabotropic glutamate receptor subtype 5 has in vivo activity and antipsychotic-like effects in rat behavioral models. J Pharmacol Exp Ther 313:199-206

Kinon BJ, Millen BA, Zhang L, and McKinzie DL (2015) Exploratory analysis for a targeted patient population responsive to the metabotropic glutamate $2 / 3$ receptor agonist pomaglumetad methionil in schizophrenia. Biol Psychiatry 78:754-762.

Kinon BJ, Zhang L, Millen BA, Osuntokun OO, Williams JE, Kollack-Walker S, Jackson K, Kryzhanovskaya L, and Jarkova N; HBBI Study Group (2011) A multicenter, inpatient, phase 2, double-blind, placebo-controlled dose-ranging study of LY2140023 monohydrate in patients with DSM-IV schizophrenia. J Clin Psychopharmacol 31:349-355.

Kinoshita A, Ohishi H, Neki A, Nomura S, Shigemoto R, Takada M, Nakanishi S, and Mizuno N (1996a) Presynaptic localization of a metabotropic glutamate receptor, mGluR8, in the rhinencephalic areas: a light and electron microscope study in the rat. Neurosci Lett 207:61-64.

Kinoshita A, Ohishi H, Nomura S, Shigemoto R, Nakanishi S, and Mizuno N (1996b) Presynaptic localization of a metabotropic glutamate receptor, mGluR4a, in the cerebellar cortex: a light and electron microscope study in the rat. Neurosci Lett 207:199-202.

Kinoshita A, Shigemoto R, Ohishi H, van der Putten H, and Mizuno N (1998) Immunohistochemical localization of metabotropic glutamate receptors, mGluR7a and mGluR7b, in the central nervous system of the adult rat and mouse: a light and electron microscopic study. J Comp Neurol 393:332-352.

Kinzie JM, Shinohara MM, van den Pol AN, Westbrook GL, and Segerson TP (1997) Immunolocalization of metabotropic glutamate receptor 7 in the rat olfactory bulb. J Comp Neurol 385:372-384.

Kitano J, Kimura K, Yamazaki Y, Soda T, Shigemoto R, Nakajima Y, and Nakanish S (2002) Tamalin, a PDZ domain-containing protein, links a protein complex formation of group 1 metabotropic glutamate receptors and the guanine nucleotide exchange factor cytohesins. J Neurosci 22:1280-1289.

Kłak K, Pałucha A, Brański P, Sowa M, and Pilc A (2007) Combined administration of PHCCC, a positive allosteric modulator of mGlu4 receptors and ACPT-I, mGlu III receptor agonist evokes antidepressant-like effects in rats. Amino Acids 32: 169-172

Klakotskaia D, Ramsey AK, Fowler SW, Serfozo P, Simonyi A, and Schachtman TR (2013) Effects of group II and III metabotropic glutamate receptor ligands on conditioned taste aversion learning. Behav Brain Res 253:9-16.

Kniazeff J, Bessis AS, Maurel D, Ansanay H, Prézeau L, and Pin JP (2004) Closed state of both binding domains of homodimeric mGlu receptors is required for full activity. Nat Struct Mol Biol 11:706-713.

Knoflach F and Kemp JA (1998) Metabotropic glutamate group II receptors activate a $\mathrm{G}$ protein-coupled inwardly rectifying $\mathrm{K}+$ current in neurones of the rat cerebellum. J Physiol 509:347-354.

Knoflach F, Mutel V, Jolidon S, Kew JN, Malherbe P, Vieira E, Wichmann J, and Kemp JA (2001) Positive allosteric modulators of metabotropic glutamate 1 receptor: characterization, mechanism of action, and binding site. Proc Natl Acad Sci USA 98:13402-13407.

Ko SJ, Isozaki K, Kim I, Lee JH, Cho HJ, Sohn SY, Oh SR, Park S, Kim DG, Kim CH, et al. (2012) PKC phosphorylation regulates mGluR5 trafficking by enhancing binding of Siah-1A. J Neurosci 32:16391-16401.

Koehl A, Hu H, Feng D, Sun B, Zhang Y, Robertson MJ, Chu M, Kobilka TS, Laeremans T, Steyaert J, et al. (2019) Structural insights into the activation of metabotropic glutamate receptors. Nature 566:79-84.

Kohara A, Toya T, Tamura S, Watabiki T, Nagakura Y, Shitaka Y, Hayashibe S, Kawabata S, and Okada M (2005) Radioligand binding properties and pharmacological characterization of 6-amino-N-cyclohexyl-N,3-dimethylthiazolo[3,2-a]benzimidazole-2carboxamide (YM-298198), a high-affinity, selective, and noncompetitive antagonist of metabotropic glutamate receptor type 1. J Pharmacol Exp Ther 315:163-169.

Koike C, Obara T, Uriu Y, Numata T, Sanuki R, Miyata K, Koyasu T, Ueno S, Funabiki K, Tani A, et al. (2010) TRPM1 is a component of the retinal ON bipolar cell transduction channel in the mGluR6 cascade. Proc Natl Acad Sci USA 107: 332-337.

Kolczewski S, Adam G, Stadler H, Mutel V, Wichmann J, and Woltering T (1999) Synthesis of heterocyclic enol ethers and their use as group 2 metabotropic glutamate receptor antagonists. Bioorg Med Chem Lett 9:2173-2176.

Konieczny J, Ossowska K, Wolfarth S, and Pilc A (1998) LY354740, a group II metabotropic glutamate receptor agonist with potential antiparkinsonian properties in rats. Naunyn Schmiedebergs Arch Pharmacol 358:500-502.

Kramer RH, Mourot A, and Adesnik H (2013) Optogenetic pharmacology for control of native neuronal signaling proteins. Nat Neurosci 16:816-823.

Kubas H, Meyer U, Hechenberger M, Klein KU, Plitt P, Zemribo R, Spexgoor HW, van Assema SG, and Abel U (2013) Scaffold hopping approach towards various AFQ-056 analogs as potent metabotropic glutamate receptor 5 negative allosteric modulators. Bioorg Med Chem Lett 23:6370-6376.

Kubo Y, Miyashita T, and Murata Y (1998) Structural basis for a Ca2+-sensing function of the metabotropic glutamate receptors. Science 279:1722-1725.

Kubota H, Nagao S, Obata K, and Hirono M (2014) mGluR1-mediated excitation of cerebellar GABAergic interneurons requires both $\mathrm{G}$ protein-dependent and SrcERK1/2-dependent signaling pathways. PLoS One 9:e106316

Kufahl PR, Hood LE, Nemirovsky NE, Barabas P, Halstengard C, Villa A, Moore E Watterson LR, and Olive MF (2012) Positive allosteric modulation of mGluR5 accelerates extinction learning but not relearning following methamphetamine self-administration. Front Pharmacol 3:194

Kumar N, Laferriere A, Yu JS, Poon T, and Coderre TJ (2010) Metabotropic glutamate receptors (mGluRs) regulate noxious stimulus-induced glutamate release in 
the spinal cord dorsal horn of rats with neuropathic and inflammatory pain. $J$ Neurochem 114:281-290.

Kumar R, Hauser RA, Mostillo J, Dronamraju N, Graf A, Merschhemke M, and Kenney C (2016) Mavoglurant (AFQ056) in combination with increased levodopa dosages in Parkinson's disease patients. Int J Neurosci 126:20-24.

Kumar V, Fahey PG, Jong YJ, Ramanan N, and O'Malley KL (2012) Activation of intracellular metabotropic glutamate receptor 5 in striatal neurons leads to upregulation of genes associated with sustained synaptic transmission including Arc/ Arg3.1 protein. J Biol Chem 287:5412-5425.

Kumar V, Jong YJ, and O'Malley KL (2008) Activated nuclear metabotropic glutamate receptor mGlu5 couples to nuclear $\mathrm{Gq} / 11$ proteins to generate inositol 1,4,5trisphosphate-mediated nuclear Ca2+ release. J Biol Chem 283:14072-14083.

Kumari R, Castillo C, and Francesconi A (2013) Agonist-dependent signaling by group I metabotropic glutamate receptors is regulated by association with lipid domains. J Biol Chem 288:32004-32019.

Kumpost J, Syrova Z, Kulihova L, Frankova D, Bologna JC, Hlavackova V, Prezeau L, Kralikova M, Hruskova B, Pin JP, et al. (2008) Surface expression of metabotropic glutamate receptor variants mGluR1a and mGluR1b in transfected HEK293 cells. Neuropharmacology 55:409-418.

Kunishima N, Shimada Y, Tsuji Y, Sato T, Yamamoto M, Kumasaka T, Nakanishi S, Jingami H, and Morikawa K (2000) Structural basis of glutamate recognition by a dimeric metabotropic glutamate receptor. Nature 407:971-977.

Kurita M, Holloway T, García-Bea A, Kozlenkov A, Friedman AK, Moreno JL, Heshmati M, Golden SA, Kennedy PJ, Takahashi N, et al. (2012) HDAC2 regulates atypical antipsychotic responses through the modulation of mGlu2 promoter activity. Nat Neurosci 15:1245-1254

Laurie DJ, Boddeke HW, Hiltscher R, and Sommer B (1996) HmGlu1d, a novel splice variant of the human type I metabotropic glutamate receptor. Eur J Pharmacol 296:R1-R3.

Lavreysen H, Ahnaou A, Drinkenburg W, Langlois X, Mackie C, Pype S, Lütjens R, Le Poul E, Trabanco AA, and Nuñez JM (2015) Pharmacological and pharmacokinetic properties of JNJ-40411813, a positive allosteric modulator of the mGlu2 receptor. Pharmacol Res Perspect 3:e00096.

Lavreysen H, Janssen C, Bischoff F, Langlois X, Leysen JE, and Lesage AS (2003) [3H]R214127: a novel high-affinity radioligand for the mGlu1 receptor reveals a common binding site shared by multiple allosteric antagonists. Mol Pharmacol 63:1082-1093.

Lavreysen H, Langlois X, Ahnaou A, Drinkenburg W, te Riele P, Biesmans I, Van der Linden I, Peeters L, Megens A, Wintmolders C, et al. (2013) Pharmacological characterization of JNJ-40068782, a new potent, selective, and systemically active positive allosteric modulator of the mGlu2 receptor and its radioligand [3H]JNJ40068782. J Pharmacol Exp Ther 346:514-527.

Lavreysen H, Wouters R, Bischoff F, Nóbrega Pereira S, Langlois X, Blokland S, Somers M, Dillen L, and Lesage AS (2004) JNJ16259685, a highly potent, selective and systemically active mGlu1 receptor antagonist. Neuropharmacology 47 961-972.

Lax NC, George DC, Ignatz C, and Kolber BJ (2014) The mGluR5 antagonist fenobam induces analgesic conditioned place preference in mice with spared nerve injury. PLoS One 9:e103524

Leach K and Gregory KJ (2017) Molecular insights into allosteric modulation of Class C G protein-coupled receptors. Pharmacol Res 116:105-118.

Leach K, Sexton PM, and Christopoulos A (2007) Allosteric GPCR modulators: taking advantage of permissive receptor pharmacology. Trends Pharmacol Sci 28:382-389.

Lebois EP (2008) Neither typical nor atypical: LY404039 provides proof of concept that selective targeting of mGluR2/3 receptors is a valid mechanism for obtaining antipsychotic efficacy. Curr Top Med Chem 8:1480-1481.

Lebourgeois S, Vilpoux C, Jeanblanc J, Acher F, Marie N, Noble F, and Naassila M (2018) Pharmacological activation of mGlu4 and mGlu7 receptors, by LSP2-9166, reduces ethanol consumption and relapse in rat. Neuropharmacology 133:163-170.

Lee J, Munguba H, Gutzeit VA, Singh DR, Kristt M, Dittman JS, and Levitz J (2020) Defining the homo- and heterodimerization propensities of metabotropic glutamate receptors. Cell Rep 31:107605.

Lee JH, Lee J, Choi KY, Hepp R, Lee JY, Lim MK, Chatani-Hinze M, Roche PA, Kim DG, Ahn YS, et al. (2008) Calmodulin dynamically regulates the trafficking of the metabotropic glutamate receptor mGluR5. Proc Natl Acad Sci USA 105: $12575-12580$.

Lee JY, Choe ES, Yang CH, Choi KH, Cheong JH, Jang CG, Seo JW, and Yoon SS (2016) The mGluR5 antagonist MPEP suppresses the expression and reinstatement, but not the acquisition, of the ethanol-conditioned place preference in mice. Pharmacol Biochem Behav 140:33-38.

Lennon SM, Rivero G, Matharu A, Howson PA, Jane DE, Roberts PJ, and Kelly E (2010) Metabotropic glutamate receptor mGlu2 is resistant to homologous agonistinduced desensitization but undergoes protein kinase C-mediated heterologous desensitization. Eur J Pharmacol 649:29-37.

Le Poul E, Boléa C, Girard F, Poli S, Charvin D, Campo B, Bortoli J, Bessif A, Luo B Koser AJ, et al. (2012) A potent and selective metabotropic glutamate receptor 4 positive allosteric modulator improves movement in rodent models of Parkinson's disease. J Pharmacol Exp Ther 343:167-177.

Lesage F, Terrenoire C, Romey G, and Lazdunski M (2000) Human TREK2, a 2 P domain mechano-sensitive $\mathrm{K}+$ channel with multiple regulations by polyunsaturated fatty acids, lysophospholipids, and Gs, Gi, and Gq protein-coupled receptors. J Biol Chem 275:28398-28405.

Levitz J, Broichhagen J, Leippe P, Konrad D, Trauner D, and Isacoff EY (2017) Dual optical control and mechanistic insights into photoswitchable group II and III metabotropic glutamate receptors. Proc Natl Acad Sci USA 114:E3546-E3554.

Levitz J, Habrian C, Bharill S, Fu Z, Vafabakhsh R, and Isacoff EY (2016) Mechanism of assembly and cooperativity of homomeric and heteromeric metabotropic glutamate receptors. Neuron 92:143-159.

Levitz J, Pantoja C, Gaub B, Janovjak H, Reiner A, Hoagland A, Schoppik D, Kane B Stawski P, Schier AF, et al. (2013) Optical control of metabotropic glutamate receptors. Nat Neurosci 16:507-516.
Li ML, Yang SS, Xing B, Ferguson BR, Gulchina Y, Li YC, Li F, Hu XQ, and Gao WJ (2015) LY395756, an mGluR2 agonist and mGluR3 antagonist, enhances NMDA receptor expression and function in the normal adult rat prefrontal cortex, but fails to improve working memory and reverse MK801-induced working memory impairment. Exp Neurol 273:190-201.

Li X, D’Souza MS, Niño AM, Doherty J, Cross A, and Markou A (2016) Attenuation of nicotine-taking and nicotine-seeking behavior by the mGlu2 receptor positive allosteric modulators AZD8418 and AZD8529 in rats. Psychopharmacology (Berl) 233:1801-1814

Li X, Li J, Gardner EL, and Xi ZX (2010) Activation of mGluR7s inhibits cocaineinduced reinstatement of drug-seeking behavior by a nucleus accumbens glutamate-mGluR2/3 mechanism in rats. $J$ Neurochem 114:1368-1380.

Li X, Li J, Peng XQ, Spiller K, Gardner EL, and Xi ZX (2009) Metabotropic glutamate receptor 7 modulates the rewarding effects of cocaine in rats: involvement of a ventra pallidal GABAergic mechanism. Neuropsychopharmacology 34:1783-1796.

Li X, Peng XQ, Jordan CJ, Li J, Bi GH, He Y, Yang HJ, Zhang HY, Gardner EL, and Xi ZX (2018) mGluR5 antagonism inhibits cocaine reinforcement and relapse by elevation of extracellular glutamate in the nucleus accumbens via a CB1 receptor mechanism. Sci Rep 8:3686

Liberatore F, Bucci D, Mascio G, Madonna M, Di Pietro P, Beneventano M, Puliti AM, Battaglia G, Bruno V, Nicoletti F, et al. (2017) Permissive role for mGlu1 metabotropic glutamate receptors in excitotoxic retinal degeneration. Neuroscience 363:142-149.

Liechti ME and Markou A (2007) Interactive effects of the mGlu5 receptor antagonist MPEP and the mGlu2/3 receptor antagonist LY341495 on nicotine selfadministration and reward deficits associated with nicotine withdrawal in rats. Eur J Pharmacol 554:164-174.

Lindemann L, Jaeschke G, Michalon A, Vieira E, Honer M, Spooren W, Porter R, Hartung T, Kolczewski S, Büttelmann B, et al. (2011) CTEP: a novel, potent, longacting, and orally bioavailable metabotropic glutamate receptor 5 inhibitor. $J$ Pharmacol Exp Ther 339:474-486.

Linden AM, Bergeron M, Baez M, and Schoepp DD (2003) Systemic administration of the potent mGlu8 receptor agonist (S)-3,4-DCPG induces c-Fos in stress-related brain regions in wild-type, but not mGlu8 receptor knockout mice. Neuropharmacology 45:473-483.

Lindsley CW, Emmitte KA, Hopkins CR, Bridges TM, Gregory KJ, Niswender CM, and Conn PJ (2016) Practical strategies and concepts in GPCR allosteric modulator discovery: recent advances with metabotropic glutamate receptors. Chem Rev 116: 6707-6741.

Lindsley CW, Wisnoski DD, Leister WH, O'brien JA, Lemaire W, Williams DL Jr., Burno M, Sur C, Kinney GG, Pettibone DJ, et al. (2004) Discovery of positive allosteric modulators for the metabotropic glutamate receptor subtype 5 from a series of $\mathrm{N}$-(1,3-diphenyl-1H- pyrazol-5-yl)benzamides that potentiate receptor function in vivo. $J$ Med Chem 47:5825-5828.

Litman RE, Smith MA, Doherty JJ, Cross A, Raines S, Gertsik L, and Zukin SR (2016) AZD8529, a positive allosteric modulator at the mGluR2 receptor, does not improve symptoms in schizophrenia: a proof of principle study. Schizophr Res $\mathbf{1 7 2}$ $152-157$.

Litschig S, Gasparini F, Rueegg D, Stoehr N, Flor PJ, Vranesic I, Prézeau L, Pin JP Thomsen C, and Kuhn R (1999) CPCCOEt, a noncompetitive metabotropic glutamate receptor 1 antagonist, inhibits receptor signaling without affecting glutamate binding. Mol Pharmacol 55:453-461.

Littman L, Chase LA, Renzi M, Garlin AB, Koerner JF, Johnson RL, and Robinson MB (1995) Effects of quisqualic acid analogs on metabotropic glutamate receptors coupled to phosphoinositide hydrolysis in rat hippocampus. Neuropharmacology 34:829-841.

Liu F, Grauer S, Kelley C, Navarra R, Graf R, Zhang G, Atkinson PJ, Popiolek M, Wantuch C, Khawaja X, et al. (2008) ADX47273 [S-(4-fluoro-phenyl)-\{3-[3-(4-fluorophenyl)-[1,2,4]-oxadiazol-5-yl]-piperidin-1-yl\}-methanone]: a novel metabotropic glutamate receptor 5 -selective positive allosteric modulator with preclinical antipsychotic-like and procognitive activities. J Pharmacol Exp Ther 327:827-839.

Liu J, Zhang Z, Moreno-Delgado D, Dalton JA, Rovira X, Trapero A, Goudet C, Llebaria A, Giraldo J, Yuan Q, et al. (2017) Allosteric control of an asymmetric transduction in a G protein-coupled receptor heterodimer. eLife 6:e26985.

Liu XH, Han M, Zhu JX, Sun N, Tang JS, Huo FQ, Li J, Xu FY, and Du JQ (2012) Metabotropic glutamate subtype 7 and 8 receptors oppositely modulate cardiac nociception in the rat nucleus tractus solitarius. Neuroscience 220:322-329.

Loane DJ, Stoica BA, Tchantchou F, Kumar A, Barrett JP, Akintola T, Xue F, Conn PJ, and Faden AI (2014) Novel mGluR5 positive allosteric modulator improves functional recovery, attenuates neurodegeneration, and alters microglial polarization after experimental traumatic brain injury. Neurotherapeutics 11:857-869.

Lopez S, Turle-Lorenzo N, Acher F, De Leonibus E, Mele A, and Amalric M (2007) Targeting group III metabotropic glutamate receptors produces complex behavioral effects in rodent models of Parkinson's disease. J Neurosci 27:6701-6711.

Lundström L, Bissantz C, Beck J, Dellenbach M, Woltering TJ, Wichmann J, and Gatti S (2016) Pharmacological and molecular characterization of the positive allosteric modulators of metabotropic glutamate receptor 2. Neuropharmacology 111:253-265.

Lundström L, Bissantz C, Beck J, Wettstein JG, Woltering TJ, Wichmann J, and Gatti S (2011) Structural determinants of allosteric antagonism at metabotropic glutamate receptor 2: mechanistic studies with new potent negative allosteric modulators. Br J Pharmacol 164:521-537.

Lundström L, Kuhn B, Beck J, Borroni E, Wettstein JG, Woltering TJ, and Gatti S (2009) Mutagenesis and molecular modeling of the orthosteric binding site of the mGlu2 receptor determining interactions of the group II receptor antagonist (3)HHYDIA. ChemMedChem 4:1086-1094.

Macek TA, Schaffhauser H, and Conn PJ (1998) Protein kinase C and A3 adenosine receptor activation inhibit presynaptic metabotropic glutamate receptor (mGluR) function and uncouple mGluRs from GTP-binding proteins. J Neurosci 18:6138-6146.

MacInnes N, Messenger MJ, and Duty S (2004) Activation of group III metabotropic glutamate receptors in selected regions of the basal ganglia alleviates akinesia in the reserpine-treated rat. $\mathrm{Br} J$ Pharmacol 141:15-22. 
Maejima T, Hashimoto K, Yoshida T, Aiba A, and Kano M (2001) Presynaptic inhibition caused by retrograde signal from metabotropic glutamate to cannabinoid receptors. Neuron 31:463-475.

Mahato PK, Pandey S, and Bhattacharyya S (2015) Differential effects of protein phosphatases in the recycling of metabotropic glutamate receptor 5. Neuroscience 306:138-150.

Maj C, Minelli A, Giacopuzzi E, Sacchetti E, and Gennarelli M (2016) The role of metabotropic glutamate receptor genes in schizophrenia. Curr Neuropharmacol 14: $540-550$

Maj M, Bruno V, Dragic Z, Yamamoto R, Battaglia G, Inderbitzin W, Stoehr N, Stein T, Gasparini F, Vranesic I, et al. (2003) (-)-PHCCC, a positive allosteric modulator of mGluR4: characterization, mechanism of action, and neuroprotection. Neuropharmacology 45:895-906.

Makoff A, Pilling C, Harrington K, and Emson P (1996) Human metabotropic glutamate receptor type 7: molecular cloning and mRNA distribution in the CNS. Brain Res Mol Brain Res 40:165-170.

Makoff AJ, Phillips T, Pilling C, and Emson P (1997) Expression of a novel splice variant of human mGluR1 in the cerebellum. Neuroreport 8:2943-2947.

Malherbe P, Kratochwil N, Knoflach F, Zenner MT, Kew JN, Kratzeisen C, Maerki HP, Adam G, and Mutel V (2003) Mutational analysis and molecular modeling of the allosteric binding site of a novel, selective, noncompetitive antagonist of the metabotropic glutamate 1 receptor. J Biol Chem 278:8340-8347.

Malherbe P, Kratzeisen C, Lundstrom K, Richards JG, Faull RL, and Mutel V (1999) Cloning and functional expression of alternative spliced variants of the human metabotropic glutamate receptor 8. Brain Res Mol Brain Res 67:201-210.

Mao L and Wang JQ (2003a) Metabotropic glutamate receptor 5-regulated Elk-1 phosphorylation and immediate early gene expression in striatal neurons. $J$ Neurochem 85:1006-1017.

Mao L and Wang JQ (2003b) Phosphorylation of cAMP response element-binding protein in cultured striatal neurons by metabotropic glutamate receptor subtype 5. J Neurochem 84:233-243.

Marabese I, Rossi F, Palazzo E, de Novellis V, Starowicz K, Cristino L, Vita D, Gatta L, Guida F, Di Marzo V, et al. (2007) Periaqueductal gray metabotropic glutamate receptor subtype 7 and 8 mediate opposite effects on amino acid release, rostral ventromedial medulla cell activities, and thermal nociception. $J$ Neurophysiol 98:43-53.

Marek GJ, Wright RA, and Schoepp DD (2006) 5-Hydroxytryptamine2A (5-HT2A) receptor regulation in rat prefrontal cortex: interaction of a phenethylamine hallucinogen and the metabotropic glutamate2/3 receptor agonist LY354740. Neurosci Lett 403:256-260.

Marek GJ, Wright RA, Schoepp DD, Monn JA, and Aghajanian GK (2000) Physiological antagonism between 5-hydroxytryptamine(2A) and group II metabotropic glutamate receptors in prefrontal cortex. J Pharmacol Exp Ther 292:76-87.

Marino MJ, Valenti O, O’Brien JA, Williams DL Jr., and Conn PJ (2003a) Modulation of inhibitory transmission in the rat globus pallidus by activation of mGluR4. Ann N Y Acad Sci 1003:435-437.

Marino MJ, Williams DL Jr., O’Brien JA, Valenti O, McDonald TP, Clements MK, Wang R, DiLella AG, Hess JF, Kinney GG, et al. (2003b) Allosteric modulation of group III metabotropic glutamate receptor 4: a potential approach to Parkinson's disease treatment. Proc Natl Acad Sci USA 100:13668-13673.

Marks CR, Shonesy BC, Wang X, Stephenson JR, Niswender CM, and Colbran RJ (2018) Activated CaMKII $\alpha$ binds to the $\mathrm{mGlu}_{5}$ metabotropic glutamate receptor and modulates calcium mobilization. Mol Pharmacol 94:1352-1362.

Marszalek-Grabska M, Gibula-Bruzda E, Bodzon-Kulakowska A, Suder P, Gawel K, Talarek S, Listos J, Kedzierska E, Danysz W, and Kotlinska JH (2018) ADX-47273, a mGlu5 receptor positive allosteric modulator, attenuates deficits in cognitive flexibility induced by withdrawal from 'binge-like' ethanol exposure in rats. Behav Brain Res 338:9-16.

Martín R, Torres M, and Sánchez-Prieto J (2007) mGluR7 inhibits glutamate release through a PKC-independent decrease in the activity of P/Q-type Ca2+ channels and by diminishing cAMP in hippocampal nerve terminals. Eur $J$ Neurosci 26 : $312-322$.

Masilamoni GJ, Bogenpohl JW, Alagille D, Delevich K, Tamagnan G, Votaw JR, Wichmann T, and Smith Y (2011) Metabotropic glutamate receptor 5 antagonist protects dopaminergic and noradrenergic neurons from degeneration in MPTPtreated monkeys. Brain 134:2057-2073.

Masu M, Iwakabe H, Tagawa Y, Miyoshi T, Yamashita M, Fukuda Y, Sasaki H, Hiroi K, Nakamura Y, and Shigemoto R (1995) Specific deficit of the ON response in visual transmission by targeted disruption of the mGluR6 gene. Cell 80:757-765.

Matrisciano F, Zusso M, Panaccione I, Turriziani B, Caruso A, Iacovelli L, Noviello L, Togna G, Melchiorri D, Debetto P, et al. (2008) Synergism between fluoxetine and the mGlu2/3 receptor agonist, LY379268, in an in vitro model for antidepressant drug-induced neurogenesis. Neuropharmacology 54:428-437.

Mazur JE (1995) Conditioned reinforcement and choice with delayed and uncertain primary reinforcers. J Exp Anal Behav 63:139-150.

Mazzitelli M, Palazzo E, Maione S, and Neugebauer V (2018) Group II metabotropic glutamate receptors: role in pain mechanisms and pain modulation. Front Mol Neurosci 11:383.

McColm J, Brittain C, Suriyapperuma S, Swanson S, Tauscher-Wisniewski S, Foster J, Soon D, and Jackson K (2017) Evaluation of single and multiple doses of a novel mGlu2 agonist, a potential antipsychotic therapy, in healthy subjects. $\mathrm{Br} J \mathrm{Clin}$ Pharmacol 83:1654-1667.

McCool BA, Pin JP, Brust PF, Harpold MM, and Lovinger DM (1996) Functional coupling of rat group II metabotropic glutamate receptors to an omega-conotoxin GVIA-sensitive calcium channel in human embryonic kidney 293 cells. Mol Pharmacol 50:912-922.

Megens AA, Hendrickx HM, Hens KA, Talloen WJ, and Lavreysen H (2014) mGlu(2) receptor-mediated modulation of conditioned avoidance behavior in rats. Eur $J$ Pharmacol 727:130-139.

Mehta MV, Gandal MJ, and Siegel SJ (2011) mGluR5-antagonist mediated reversal of elevated stereotyped, repetitive behaviors in the VPA model of autism. PLoS One 6: 26077 .
Meldrum BS (2000) Glutamate as a neurotransmitter in the brain: review of physiology and pathology. J Nutr 130 (Suppl):1007S-1015S.

Metcalf CS, Klein BD, Smith MD, Pruess T, Ceusters M, Lavreysen H, Pype S, Van Osselaer N, Twyman R, and White HS (2017) Efficacy of mGlu $_{2}$-positive allosteric modulators alone and in combination with levetiracetam in the mouse $6 \mathrm{~Hz}$ model of psychomotor seizures. Epilepsia 58:484-493.

Michalon A, Bruns A, Risterucci C, Honer M, Ballard TM, Ozmen L, Jaeschke G, Wettstein JG, von Kienlin M, Künnecke B, et al. (2014) Chronic metabotropic glutamate receptor 5 inhibition corrects local alterations of brain activity and improves cognitive performance in fragile X mice. Biol Psychiatry 75:189-197.

Michalon A, Sidorov M, Ballard TM, Ozmen L, Spooren W, Wettstein JG, Jaeschke G, Bear MF, and Lindemann L (2012) Chronic pharmacological mGlu5 inhibition corrects fragile $\mathrm{X}$ in adult mice. Neuron 74:49-56.

Micheli F, Fabio RD, Cavanni P, Rimland JM, Capelli AM, Chiamulera C, Corsi M, Corti C, Donati D, Feriani A, et al. (2003) Synthesis and pharmacological characterisation of 2,4-dicarboxy-pyrroles as selective non-competitive mGluR1 antagonists. Bioorg Med Chem 11:171-183.

Millán C, Luján R, Shigemoto R, and Sánchez-Prieto J (2002a) The inhibition of glutamate release by metabotropic glutamate receptor 7 affects both $[\mathrm{Ca} 2+] \mathrm{c}$ and cAMP: evidence for a strong reduction of $\mathrm{Ca} 2+$ entry in single nerve terminals. J Biol Chem 277:14092-14101.

Millán C, Luján R, Shigemoto R, and Sánchez-Prieto J (2002b) Subtype-specific expression of group III metabotropic glutamate receptors and $\mathrm{Ca} 2+$ channels in single nerve terminals. J Biol Chem 277:47796-47803.

Minakami R, Iida K, Hirakawa N, and Sugiyama H (1995) The expression of two splice variants of metabotropic glutamate receptor subtype 5 in the rat brain and neuronal cells during development. J Neurochem 65:1536-1542.

Minakami R, Katsuki F, and Sugiyama H (1993) A variant of metabotropic glutamate receptor subtype 5: an evolutionally conserved insertion with no termination codon. Biochem Biophys Res Commun 194:622-627.

Minakami R, Katsuki F, Yamamoto T, Nakamura K, and Sugiyama H (1994) Molecular cloning and the functional expression of two isoforms of human metabotropic glutamate receptor subtype 5. Biochem Biophys Res Commun 199:1136-1143.

Mion S, Corti C, Neki A, Shigemoto R, Corsi M, Fumagalli G, and Ferraguti F (2001) Bidirectional regulation of neurite elaboration by alternatively spliced metabotropic glutamate receptor 5 (mGluR5) isoforms. Mol Cell Neurosci 17:957-972.

Mitsukawa K, Yamamoto R, Ofner S, Nozulak J, Pescott O, Lukic S, Stoehr N, Mombereau C, Kuhn R, McAllister KH, et al. (2005) A selective metabotropic glutamate receptor 7 agonist: activation of receptor signaling via an allosteric site modulates stress parameters in vivo. Proc Natl Acad Sci USA 102:18712-18717.

Miyamoto M, Ishida M, and Shinozaki H (1997) Anticonvulsive and neuroprotective actions of a potent agonist (DCG-IV) for group II metabotropic glutamate receptors against intraventricular kainate in the rat. Neuroscience 77:131-140.

Miyashita T and Kubo Y (2000a) Extracellular Ca2+ sensitivity of mGluR1alpha associated with persistent glutamate response in transfected $\mathrm{CHO}$ cells. Receptors Channels 7:25-40.

Miyashita T and Kubo Y (2000b) Extracellular Ca2+ sensitivity of mGluR1alpha induces an increase in the basal cAMP level by direct coupling with Gs protein in transfected CHO cells. Receptors Channels 7:77-91.

Moldrich RX, Apricó K, Diwakarla S, O’Shea RD, and Beart PM (2002) Astrocyte $\mathrm{mGlu}(2 / 3)$-mediated cAMP potentiation is calcium sensitive: studies in murine neuronal and astrocyte cultures. Neuropharmacology 43:189-203.

Molinaro G, Traficante A, Riozzi B, Di Menna L, Curto M, Pallottino S, Nicoletti F, Bruno V, and Battaglia G (2009) Activation of mGlu2/3 metabotropic glutamate receptors negatively regulates the stimulation of inositol phospholipid hydrolysis mediated by 5 -hydroxytryptamine2A serotonin receptors in the frontal cortex of living mice. Mol Pharmacol 76:379-387.

Moloney RD, Golubeva AV, O'Connor RM, Kalinichev M, Dinan TG, and Cryan JF (2015) Negative allosteric modulation of the mGlu7 receptor reduces visceral hypersensitivity in a stress-sensitive rat strain. Neurobiol Stress 2:28-33.

Monn JA, Valli MJ, Massey SM, Hansen MM, Kress TJ, Wepsiec JP, Harkness AR Grutsch JL Jr., Wright RA, Johnson BG, et al. (1999) Synthesis, pharmacological characterization, and molecular modeling of heterobicyclic amino acids related to (+)-2-aminobicyclo[3.1.0] hexane-2,6-dicarboxylic acid (LY354740): identification of two new potent, selective, and systemically active agonists for group II metabotropic glutamate receptors. J Med Chem 42:1027-1040.

Monn JA, Henry SS, Massey SM, Clawson DK, Chen Q, Diseroad BA, Bhardwaj RM, Atwell S, Lu F, Wang J, et al. (2018) Synthesis and pharmacological characterization of $\mathrm{C} 4_{\beta}$-amide-substituted 2-aminobicyclo[3.1.0]hexane-2,6-dicarboxylates. Identification of (1 S,2 S,4 S,5 R,6 S)-2-amino-4-[(3-methoxybenzoyl)aminolbicyclo [3.1.0] hexane-2,6-dicarboxylic acid (LY2794193), a highly potent and selective $\mathrm{mGlu}_{3}$ receptor agonist. J Med Chem 61:2303-2328.

Monn JA, Prieto L, Taboada L, Hao J, Reinhard MR, Henry SS, Beadle CD, Walton L, Man T, Rudyk H, et al. (2015a) Synthesis and pharmacological characterization of C4-(Thiotriazolyl)-substituted-2-aminobicyclo[3.1.0]hexane-2,6-dicarboxylates. Identification of (1R,2S,4R,5R,6R)-2-amino-4-(1H-1,2,4-triazol-3-ylsulfanyl)bicyclo [3.1.0]hexane-2,6-dicarboxylic acid (LY2812223), a highly potent, functionally selective mGlu2 receptor agonist. J Med Chem 58:7526-7548.

Monn JA, Prieto L, Taboada L, Pedregal C, Hao J, Reinhard MR, Henry SS, Goldsmith PJ, Beadle CD, Walton L, et al. (2015b) Synthesis and pharmacological characterization of C4-disubstituted analogs of 1S,2S,5R,6S-2-aminobicyclo[3.1.0] hexane-2,6-dicarboxylate: identification of a potent, selective metabotropic glutamate receptor agonist and determination of agonist-bound human mGlu2 and mGlu3 amino terminal domain structures. $J$ Med Chem 58:1776-1794.

Monn JA, Valli MJ, Massey SM, Wright RA, Salhoff CR, Johnson BG, Howe T, Alt CA, Rhodes GA, Robey RL, et al. (1997) Design, synthesis, and pharmacological characterization of (+)-2-aminobicyclo[3.1.0]hexane-2,6-dicarboxylic acid (LY354740): a potent, selective, and orally active group 2 metabotropic glutamate receptor agonist possessing anticonvulsant and anxiolytic properties. J Med Chem 40:528-537.

Montana MC, Cavallone LF, Stubbert KK, Stefanescu AD, Kharasch ED, and Gereau RW IV (2009) The metabotropic glutamate receptor subtype 5 antagonist fenobam 
is analgesic and has improved in vivo selectivity compared with the prototypical antagonist 2-methyl-6-(phenylethynyl)-pyridine. J Pharmacol Exp Ther 330: 834-843.

Montana MC, Conrardy BA, Cavallone LF, Kolber BJ, Rao LK, Greco SC, and Gereau RW IV (2011) Metabotropic glutamate receptor 5 antagonism with fenobam: examination of analgesic tolerance and side effect profile in mice. Anesthesiology 115 : $1239-1250$.

Morè L, Gravius A, Pietraszek M, Belozertseva I, Malyshkin A, Shekunova E, Barberi C, Schaefer D, Schmidt WJ, and Danysz W (2007) Comparison of the mGluR1 antagonist A-841720 in rat models of pain and cognition. Behav Pharmacol 18:273-281.

Moreno JL, Holloway T, Rayannavar V, Sealfon SC, and González-Maeso J (2013) Chronic treatment with LY341495 decreases 5-HT(2A) receptor binding and hallucinogenic effects of LSD in mice. Neurosci Lett 536:69-73.

Moreno JL, Miranda-Azpiazu P, García-Bea A, Younkin J, Cui M, Kozlenkov A, BenEzra A, Voloudakis G, Fakira AK, Baki L, et al. (2016) Allosteric signaling through an mGlu2 and 5-HT2A heteromeric receptor complex and its potential contribution to schizophrenia. Sci Signal 9:ra5.

Moreno Delgado D, Møller TC, Ster J, Giraldo J, Maurel D, Rovira X, Scholler P, Zwier JM, Perroy J, Durroux T, et al. (2017) Pharmacological evidence for a metabotropic glutamate receptor heterodimer in neuronal cells. eLife 6:e25233.

Moroni F, Lombardi G, Thomsen C, Leonardi P, Attucci S, Peruginelli F, Torregrossa SA, Pellegrini-Giampietro DE, Luneia R, and Pellicciari R (1997) Pharmacological characterization of 1-aminoindan-1,5-dicarboxylic acid, a potent mGluR1 antagonist. J Pharmacol Exp Ther 281:721-729.

Motolese M, Mastroiacovo F, Cannella M, Bucci D, Gaglione A, Riozzi B, Lütjens R, Poli SM, Celanire S, Bruno V, et al. (2015) Targeting type-2 metabotropic glutamate receptors to protect vulnerable hippocampal neurons against ischemic damage. Mol Brain 8:66.

Moult PR, Gladding CM, Sanderson TM, Fitzjohn SM, Bashir ZI, Molnar E, and Collingridge GL (2006) Tyrosine phosphatases regulate AMPA receptor trafficking during metabotropic glutamate receptor-mediated long-term depression. $J$ Neurosci 26:2544-2554.

Moyanova SG, Mastroiacovo F, Kortenska LV, Mitreva RG, Fardone E, Santolini I, Sobrado M, Battaglia G, Bruno V, Nicoletti F, et al. (2011) Protective role for type 4 metabotropic glutamate receptors against ischemic brain damage. J Cereb Blood Flow Metab 31:1107-1118.

Mundell SJ, Pula G, More JC, Jane DE, Roberts PJ, and Kelly E (2004) Activation of cyclic AMP-dependent protein kinase inhibits the desensitization and internalization of metabotropic glutamate receptors $1 \mathrm{a}$ and 1b. Mol Pharmacol 65:1507-1516.

Murat S, Bigot M, Chapron J, Konig GM, Kostenis E, Battaglia G, Nicoletti F, Bourinet E, Bockaert J, Marin P, et al. (2019) 5-HT2A receptor-dependent phosphorylation of mGlu2 receptor at Serine 843 promotes mGlu2 receptor-operated Gi/ o signaling. Mol Psychiatry 24:1610-1626.

Mutel V, Ellis GJ, Adam G, Chaboz S, Nilly A, Messer J, Bleuel Z, Metzler V, Malherbe P, Schlaeger EJ, et al. (2000) Characterization of $[(3) \mathrm{H}]$ Quisqualate binding to recombinant rat metabotropic glutamate $1 \mathrm{a}$ and $5 \mathrm{a}$ receptors and to rat and human brain sections. J Neurochem 75:2590-2601.

Muto T, Tsuchiya D, Morikawa K, and Jingami H (2007) Structures of the extracellular regions of the group II/III metabotropic glutamate receptors. Proc Natl Acad Sci USA 104:3759-3764.

Nadlewska A, Car H, Oksztel R, and Wiśniewski K (2002) Effect of (S)-3,5-DHPG on learning, exploratory activity and anxiety in rats with experimental hypoxia. Pol J Pharmacol 54:11-18.

Nakajima Y, Iwakabe H, Akazawa C, Nawa H, Shigemoto R, Mizuno N, and Nakanishi S (1993) Molecular characterization of a novel retinal metabotropic glutamate receptor mGluR6 with a high agonist selectivity for L-2-amino-4-phosphonobutyrate. J Biol Chem 268:11868-11873.

Nakamura M, Kawakita Y, Yasuhara A, Fukasawa Y, Yoshida K, Sakagami K, and Nakazato A (2006) In vitro and in vivo evaluation of the metabolism and bioavailability of ester prodrugs of mgs0039 (3-(3,4-dichlorobenzyloxy)-2-amino-6 fluorobicyclo[3.1.0] hexane-2,6-dicarboxylic acid), a potent metabotropic glutamate receptor antagonist. Drug Metab Dispos 34:369-374.

Namkoong J, Martino JJ, and Chen S (2006) From existing therapies to novel targets: a current view on melanoma. Front Biosci 11:2081-2092.

Naples MA and Hampson DR (2001) Pharmacological profiles of the metabotropic glutamate receptor ligands. Neuropharmacology 40:170-177.

Nardecchia F, Orlando R, Iacovelli L, Colamartino M, Fiori E, Leuzzi V, Piccinin S, Nistico R, Puglisi-Allegra S, Di Menna L, et al. (2018) Targeting mGlu5 metabotropic glutamate receptors in the treatment of cognitive dysfunction in a mouse model of phenylketonuria. Front Neurosci 12:154.

Nasrallah C, Rottier K, Marcellin R, Compan V, Font J, Llebaria A, Pin JP, Banères JL, and Lebon G (2018) Direct coupling of detergent purified human mGlu $\mathrm{re}_{5}$ ceptor to the heterotrimeric G proteins Gq and Gs. Sci Rep 8:4407.

Neto A and Ceol CJ (2018) Melanoma-associated GRM3 variants dysregulate melanosome trafficking and cAMP signaling. Pigment Cell Melanoma Res 31:115-119.

Neugebauer V (2002) Metabotropic glutamate receptors--important modulators of nociception and pain behavior. Pain 98:1-8.

Neugebauer V, Chen PS, and Willis WD (1999) Role of metabotropic glutamate receptor subtype mGluR1 in brief nociception and central sensitization of primate STT cells. J Neurophysiol 82:272-282.

Ngomba RT, Ferraguti F, Badura A, Citraro R, Santolini I, Battaglia G, Bruno V, De Sarro G, Simonyi A, van Luijtelaar G, et al. (2008) Positive allosteric modulation of metabotropic glutamate 4 (mGlu4) receptors enhances spontaneous and evoked absence seizures. Neuropharmacology 54:344-354.

Nickols HH, Yuh JP, Gregory KJ, Morrison RD, Bates BS, Stauffer SR, Emmitte KA, Bubser M, Peng W, Nedelcovych MT, et al. (2016) VU0477573: partial negative allosteric modulator of the subtype 5 metabotropic glutamate receptor with in vivo efficacy. J Pharmacol Exp Ther 356:123-136.

Nicodemo AA, Pampillo M, Ferreira LT, Dale LB, Cregan T, Ribeiro FM, and Ferguson SS (2010) Pyk2 uncouples metabotropic glutamate receptor G protein signaling but facilitates ERK1/2 activation. Mol Brain 3:4.
Nicoletti F, Orlando R, Di Menna L, Cannella M, Notartomaso S, Mascio G, Iacovelli L, Matrisciano F, Fazio F, Caraci F, et al. (2019) Targeting mGlu receptors for optimization of antipsychotic activity and disease-modifying effect in schizophrenia. Front Psychiatry 10:49.

Nicoletti F, Wroblewski JT, Iadarola MJ, and Costa E (1986) Serine-O-phosphate, an endogenous metabolite, inhibits the stimulation of inositol phospholipid hydrolysis elicited by ibotenic acid in rat hippocampal slices. Neuropharmacology 25:335-338.

Nikiforuk A, Popik P, Drescher KU, van Gaalen M, Relo AL, Mezler M, Marek G, Schoemaker H, Gross G, and Bespalov A (2010) Effects of a positive allosteric modulator of group II metabotropic glutamate receptors, LY487379, on cognitive flexibility and impulsive-like responding in rats. $J$ Pharmacol Exp Ther 335: $665-673$.

Nishi A, Liu F, Matsuyama S, Hamada M, Higashi H, Nairn AC, and Greengard P (2003) Metabotropic mGlu5 receptors regulate adenosine A2A receptor signaling. Proc Natl Acad Sci USA 100:1322-1327.

Niswender CM and Conn PJ (2010) Metabotropic glutamate receptors: physiology, pharmacology, and disease. Annu Rev Pharmacol Toxicol 50:295-322.

Niswender CM, Johnson KA, Miller NR, Ayala JE, Luo Q, Williams R, Saleh S, Orton D, Weaver CD, and Conn PJ (2010) Context-dependent pharmacology exhibited by negative allosteric modulators of metabotropic glutamate receptor 7. Mol Pharmacol 77:459-468.

Niswender CM, Johnson KA, Weaver CD, Jones CK, Xiang Z, Luo Q, Rodriguez AL, Marlo JE, de Paulis T, Thompson AD, et al. (2008a) Discovery, characterization, and antiparkinsonian effect of novel positive allosteric modulators of metabotropic glutamate receptor 4. Mol Pharmacol 74:1345-1358.

Niswender CM, Jones CK, Lin X, Bubser M, Thompson Gray A, Blobaum AL, Engers DW, Rodriguez AL, Loch MT, Daniels JS, et al. (2016) Development and antiparkinsonian activity of VU0418506, a selective positive allosteric modulator of metabotropic glutamate receptor 4 homomers without activity at mGlu2/4 heteromers. ACS Chem Neurosci 7:1201-1211.

Niswender CM, Lebois EP, Luo Q, Kim K, Muchalski H, Yin H, Conn PJ, and Lindsley CW (2008b) Positive allosteric modulators of the metabotropic glutamate receptor subtype 4 (mGluR4): Part I. Discovery of pyrazolo[3,4-d]pyrimidines as novel mGluR4 positive allosteric modulators. Bioorg Med Chem Lett 18:5626-5630.

Noetzel MJ, Gregory KJ, Vinson PN, Manka JT, Stauffer SR, Lindsley CW, Niswender CM, Xiang Z, and Conn PJ (2013) A novel metabotropic glutamate receptor 5 positive allosteric modulator acts at a unique site and confers stimulus bias to mGlu5 signaling. Mol Pharmacology 83:835-847.

Noetzel MJ, Rook JM, Vinson PN, Cho HP, Days E, Zhou Y, Rodriguez AL, Lavreysen H, Stauffer SR, Niswender CM, et al. (2012) Functional impact of allosteric agonist activity of selective positive allosteric modulators of metabotropic glutamate receptor subtype 5 in regulating central nervous system function. Mol Pharmacol 81: $120-133$.

Nomura A, Shigemoto R, Nakamura Y, Okamoto N, Mizuno N, and Nakanishi S (1994) Developmentally regulated postsynaptic localization of a metabotropic glutamate receptor in rat rod bipolar cells. Cell 77:361-369.

O'Brien DE, Shaw DM, Cho HP, Cross AJ, Wesolowski SS, Felts AS, Bergare J, Elmore CS, Lindsley CW, Niswender CM, et al. (2018) Differential pharmacology and binding of $\mathrm{mGlu}_{2}$ receptor allosteric modulators. Mol Pharmacol 93:526-540.

O'Brien JA, Lemaire W, Chen TB, Chang RS, Jacobson MA, Ha SN, Lindsley CW, Schaffhauser HJ, Sur C, Pettibone DJ, et al. (2003) A family of highly selective allosteric modulators of the metabotropic glutamate receptor subtype 5 . Mol Pharmacol 64:731-740.

O'Brien JA, Lemaire W, Wittmann M, Jacobson MA, Ha SN, Wisnoski DD, Lindsley CW, Schaffhauser HJ, Rowe B, Sur C, et al. (2004) A novel selective allosteric modulator potentiates the activity of native metabotropic glutamate receptor subtype 5 in rat forebrain. J Pharmacol Exp Ther 309:568-577.

O'Connor E, Allen LE, Bradshaw K, Boylan J, Moore AT, and Trump D (2006) Congenital stationary night blindness associated with mutations in GRM6 encoding glutamate receptor MGluR6. $\mathrm{Br} \mathrm{J}$ Ophthalmol 90:653-654.

O'Connor RM and Cryan JF (2013) The effects of $\mathrm{mGlu}_{7}$ receptor modulation in behavioural models sensitive to antidepressant action in two mouse strains. Behav Pharmacol 24:105-113.

O'Connor RM, Finger BC, Flor PJ, and Cryan JF (2010) Metabotropic glutamate receptor 7: at the interface of cognition and emotion. Eur J Pharmacol 639:123-131.

Okamoto N, Hori S, Akazawa C, Hayashi Y, Shigemoto R, Mizuno N, and Nakanishi S (1994) Molecular characterization of a new metabotropic glutamate receptor mGluR7 coupled to inhibitory cyclic AMP signal transduction. J Biol Chem 269:1231-1236.

Olivero G, Bonfiglio T, Vergassola M, Usai C, Riozzi B, Battaglia G, Nicoletti F, and Pittaluga A (2017) Immuno-pharmacological characterization of group II metabotropic glutamate receptors controlling glutamate exocytosis in mouse cortex and spinal cord. Br J Pharmacol 174:4785-4796.

Ornstein PL, Arnold MB, Bleisch TJ, Wright RA, Wheeler WJ, and Schoepp DD (1998) [3H]LY341495, a highly potent, selective and novel radioligand for labeling Group II metabotropic glutamate receptors. Bioorg Med Chem Lett 8:1919-1922. Ossowska K, Konieczny J, Wardas J, Pietraszek M, Kuter K, Wolfarth S, and Pilc A (2007) An influence of ligands of metabotropic glutamate receptor subtypes on parkinsonian-like symptoms and the striatopallidal pathway in rats. Amino Acids 32:179-188

Otani S, Daniel H, Takita M, and Crépel F (2002) Long-term depression induced by postsynaptic group II metabotropic glutamate receptors linked to phospholipase C and intracellular calcium rises in rat prefrontal cortex. $J$ Neurosci 22:3434-3444

Page G, Khidir FA, Pain S, Barrier L, Fauconneau B, Guillard O, Piriou A, and Hugon J (2006) Group I metabotropic glutamate receptors activate the p70S6 kinase via both mammalian target of rapamycin (mTOR) and extracellular signalregulated kinase (ERK 1/2) signaling pathways in rat striatal and hippocampal synaptoneurosomes. Neurochem Int 49:413-421.

Palazzo E, Fu Y, Ji G, Maione S, and Neugebauer V (2008) Group III mGluR7 and mGluR8 in the amygdala differentially modulate nocifensive and affective pain behaviors. Neuropharmacology 55:537-545. 
Palazzo E, Marabese I, de Novellis V, Rossi F, and Maione S (2016) Metabotropic glutamate receptor 7: from synaptic function to therapeutic implications. Curr Neuropharmacol 14:504-513.

Palazzo E, Romano R, Luongo L, Boccella S, De Gregorio D, Giordano ME, Rossi F, Marabese I, Scafuro MA, de Novellis V, et al. (2015) MMPIP, an mGluR7-selective negative allosteric modulator, alleviates pain and normalizes affective and cognitive behavior in neuropathic mice. Pain 156:1060-1073.

Palucha A, Klak K, Branski P, van der Putten H, Flor PJ, and Pilc A (2007) Activation of the mGlu7 receptor elicits antidepressant-like effects in mice. Psychopharmacology (Berl) 194:555-562.

Pałucha A, Tatarczyńska E, Brański P, Szewczyk B, Wierońska JM, Kłak K, Chojnacka-Wójcik E, Nowak G, and Pilc A (2004) Group III mGlu receptor agonists produce anxiolytic- and antidepressant-like effects after central administration in rats. Neuropharmacology 46:151-159.

Pałucha-Poniewiera A, Kłodzińska A, Stachowicz K, Tokarski K, Hess G, Schann S, Frauli M, Neuville P, and Pilc A (2008) Peripheral administration of group III mGlu receptor agonist ACPT-I exerts potential antipsychotic effects in rodents. Neuropharmacology 55:517-524

Pałucha-Poniewiera A and Pilc A (2013) A selective mGlu7 receptor antagonist MMPIP reversed antidepressant-like effects of AMN082 in rats. Behav Brain Res 238:109-112.

Panaccione I, Iacovelli L, di Nuzzo L, Nardecchia F, Mauro G, Janiri D, De Blasi A, Sani G, Nicoletti F, and Orlando R (2017) Paradoxical sleep deprivation in rats causes a selective reduction in the expression of type- 2 metabotropic glutamate receptors in the hippocampus. Pharmacol Res 117:46-53.

Pandey S, Mahato PK, and Bhattacharyya S (2014) Metabotropic glutamate receptor 1 recycles to the cell surface in protein phosphatase 2A-dependent manner in nonneuronal and neuronal cell lines. $J$ Neurochem 131:602-614.

Pandya NJ, Klaassen RV, van der Schors RC, Slotman JA, Houtsmuller A, Smit AB, and $\mathrm{Li} \mathrm{KW} \mathrm{(2016)} \mathrm{Group} 1$ metabotropic glutamate receptors 1 and 5 form a protein complex in mouse hippocampus and cortex. Proteomics 16:2698-2705.

Park EH, Lee SW, Moon SW, Suh HR, Kim YI, and Han HC (2019) Activation of peripheral group III metabotropic glutamate receptors inhibits pain transmission by decreasing neuronal excitability in the CFA-inflamed knee joint. Neurosci Lett 694:111-115.

Parmentier-Batteur S, Hutson PH, Menzel K, Uslaner JM, Mattson BA, O'Brien JA, Magliaro BC, Forest T, Stump CA, Tynebor RM, et al. (2014) Mechanism based neurotoxicity of mGlu5 positive allosteric modulators--development challenges for a promising novel antipsychotic target. Neuropharmacology 82:161-173.

Parmentier-Batteur S, O'Brien JA, Doran S, Nguyen SJ, Flick RB, Uslaner JM, Chen H, Finger EN, Williams TM, Jacobson MA, et al. (2012) Differential effects of the mGluR5 positive allosteric modulator CDPPB in the cortex and striatum following repeated administration. Neuropharmacology 62:1453-1460.

Patel JB, Martin C, and Malick JB (1982) Differential antagonism of the anticonflict effects of typical and atypical anxiolytics. Eur J Pharmacol 86:295-298.

Patil ST, Zhang L, Martenyi F, Lowe SL, Jackson KA, Andreev BV, Avedisova AS, Bardenstein LM, Gurovich IY, Morozova MA, et al. (2007) Activation of mGlu2/3 receptors as a new approach to treat schizophrenia: a randomized Phase 2 clinical trial. Nat Med 13:1102-1107.

Pecknold JC, McClure DJ, Appeltauer L, Wrzesinski L, and Allan T (1982) Treatment of anxiety using fenobam (a nonbenzodiazepine) in a double-blind standard (diazepam) placebo-controlled study. J Clin Psychopharmacol 2:129-133.

Pekhletski R, Gerlai R, Overstreet LS, Huang XP, Agopyan N, Slater NT, AbramowNewerly W, Roder JC, and Hampson DR (1996) Impaired cerebellar synaptic plasticity and motor performance in mice lacking the mGluR4 subtype of metabotropic glutamate receptor. $J$ Neurosci 16:6364-6373.

Pellegrini-Giampietro DE, Cozzi A, Peruginelli F, Leonardi P, Meli E, Pellicciari R, and Moroni F (1999) 1-Aminoindan-1,5-dicarboxylic acid and (S)-(+)-2-(3'-carboxybicyclo[1.1.1] pentyl)-glycine, two mGlu1 receptor-preferring antagonists, reduce neuronal death in in vitro and in vivo models of cerebral ischaemia. Eur J Neurosci 11:3637-3647.

Pellicciari R, Luneia R, Costantino G, Marinozzi M, Natalini B, Jakobsen P, Kanstrup A, Lombardi G, Moroni F, and Thomsen C (1995) 1-Aminoindan-1,5dicarboxylic acid: a novel antagonist at phospholipase C-linked metabotropic glutamate receptors. J Med Chem 38:3717-3719.

Pereira V and Goudet C (2019) Emerging trends in pain modulation by metabotropic glutamate receptors. Front Mol Neurosci 11:464.

Perez-Benito L, Doornbos MLJ, Cordomi A, Peeters L, Lavreysen H, Pardo L, and Tresadern G (2017) Molecular switches of allosteric modulation of the metabotropic glutamate 2 receptor. Structure 25:1153-1162.e4.

Perez-Garcia G, De Gasperi R, Gama Sosa MA, Perez GM, Otero-Pagan A, Tschiffely A, McCarron RM, Ahlers ST, Elder GA, and Gandy S (2018) PTSD-related behavioral traits in a rat model of blast-induced mTBI are reversed by the mGluR2/3 receptor antagonist BCI-838. eNeuro 5:ENEURO.0357-17.2018.

Perroy J, El Far O, Bertaso F, Pin JP, Betz H, Bockaert J, and Fagni L (2002) PICK1 is required for the control of synaptic transmission by the metabotropic glutamate receptor 7. EMBO J 21:2990-2999.

Perroy J, Prezeau L, De Waard M, Shigemoto R, Bockaert J, and Fagni L (2000) Selective blockade of $\mathrm{P} / \mathrm{Q}$-type calcium channels by the metabotropic glutamate receptor type 7 involves a phospholipase C pathway in neurons. J Neurosci 20:7896-7904.

Perry CJ, Reed F, Zbukvic IC, Kim JH, and Lawrence AJ (2016) The metabotropic glutamate 5 receptor is necessary for extinction of cocaine-associated cues. $\mathrm{Br}$ $J$ Pharmacol 173:1085-1094.

Peterlik D, Stangl C, Bauer A, Bludau A, Keller J, Grabski D, Killian T, Schmidt D, Zajicek F, Jaeschke G, et al. (2017) Blocking metabotropic glutamate receptor subtype 5 relieves maladaptive chronic stress consequences. Brain Behav Immun 59:79-92.

Pietraszek M, Rogóz Z, Wolfarth S, and Ossowska K (2004) Opposite influence of MPEP, an mGluR5 antagonist, on the locomotor hyperactivity induced by PCP and amphetamine. J Physiol Pharmacol 55:587-593.

Pin JP and Bettler B (2016) Organization and functions of mGlu and GABA $\mathrm{B}_{\mathrm{B}}$ receptor complexes. Nature 540:60-68.
Pin JP, De Colle C, Bessis AS, and Acher F (1999) New perspectives for the development of selective metabotropic glutamate receptor ligands. Eur J Pharmacol 375:277-294.

Pin JP, Waeber C, Prezeau L, Bockaert J, and Heinemann SF (1992) Alternative splicing generates metabotropic glutamate receptors inducing different patterns of calcium release in Xenopus oocytes. Proc Natl Acad Sci USA 89:10331-10335.

Pitsch J, Schoch S, Gueler N, Flor PJ, van der Putten H, and Becker AJ (2007) Functional role of mGluR1 and mGluR4 in pilocarpine-induced temporal lobe epilepsy. Neurobiol Dis 26:623-633.

Pitsikas N, Kaffe E, and Markou A (2012) The metabotropic glutamate 2/3 receptor antagonist LY341495 differentially affects recognition memory in rats. Behav Brain Res 230:374-379.

Pittaluga A (2016) Presynaptic release-regulating mGlu1 receptors in central nervous system. Front Pharmacol 7:295

Pittolo S, Gómez-Santacana X, Eckelt K, Rovira X, Dalton J, Goudet C, Pin JP, Llobet A, Giraldo J, Llebaria A, et al. (2014) An allosteric modulator to control endogenous G protein-coupled receptors with light. Nat Chem Biol 10:813-815.

Pizzi M, Benarese M, Boroni F, Goffi F, Valerio A, and Spano PF (2000) Neuroprotection by metabotropic glutamate receptor agonists on kainate-induced degeneration of motor neurons in spinal cord slices from adult rat. Neuropharmacology 39:903-910.

Platt DM, Rowlett JK, and Spealman RD (2008) Attenuation of cocaine selfadministration in squirrel monkeys following repeated administration of the mGluR5 antagonist MPEP: comparison with dizocilpine. Psychopharmacology (Berl) 200:167-176.

Podkowa K, Pilc A, Podkowa A, Sałat K, Marciniak M, and Pałucha-Poniewiera A (2018) The potential antidepressant action and adverse effects profile of scopolamine co-administered with the mGlu7 receptor allosteric agonist AMN082 in mice. Neuropharmacology 141:214-222.

Podkowa K, Pochwat B, Brański P, Pilc A, and Pałucha-Poniewiera A (2016) Group II mGlu receptor antagonist LY341495 enhances the antidepressant-like effects of ketamine in the forced swim test in rats. Psychopharmacology (Berl) 233 2901-2914.

Podkowa K, Rzeźniczek S, Marciniak M, Acher F, Pilc A, and Pałucha-Poniewiera A (2015) A novel mGlu4 selective agonist LSP4-2022 increases behavioral despair in mouse models of antidepressant action. Neuropharmacology 97:338-345.

Ponnazhagan R, Harms AS, Thome AD, Jurkuvenaite A, Gogliotti R, Niswender CM, Conn PJ, and Standaert DG (2016) The metabotropic glutamate receptor 4 positive allosteric modulator ADX88178 inhibits inflammatory responses in primary microglia. J Neuroimmune Pharmacol 11:231-237.

Popoli P, Pèzzola A, Torvinen M, Reggio R, Pintor A, Scarchilli L, Fuxe K, and Ferré S (2001) The selective mGlu(5) receptor agonist CHPG inhibits quinpirole-induced turning in 6-hydroxydopamine-lesioned rats and modulates the binding characteristics of dopamine $\mathrm{D}(2)$ receptors in the rat striatum: interactions with adenosine A(2a) receptors. Neuropsychopharmacology 25:505-513.

Porter RH, Jaeschke G, Spooren W, Ballard TM, Büttelmann B, Kolczewski S, Peters JU, Prinssen E, Wichmann J, Vieira E, et al. (2005) Fenobam: a clinically validated nonbenzodiazepine anxiolytic is a potent, selective, and noncompetitive mGlu5 receptor antagonist with inverse agonist activity. J Pharmacol Exp Ther 315: $711-721$.

Poutiainen P, Kil KE, Zhang Z, Kuruppu D, Tannous B, and Brownell AL (2015) Cooperative binding assay for the characterization of mGlu4 allosteric modulators. Neuropharmacology 97:142-148.

Prickett TD, Wei X, Cardenas-Navia I, Teer JK, Lin JC, Walia V, Gartner J, Jiang J, Cherukuri PF, Molinolo A, et al. (2011) Exon capture analysis of G protein-coupled receptors identifies activating mutations in GRM3 in melanoma. Nat Genet 43: $1119-1126$

Pula G, Mundell SJ, Roberts PJ, and Kelly E (2004) Agonist-independent internalization of metabotropic glutamate receptor 1a is arrestin- and clathrin-dependent and is suppressed by receptor inverse agonists. $J$ Neurochem 89:1009-1020.

Purgert CA, Izumi Y, Jong YJ, Kumar V, Zorumski CF, and O'Malley KL (2014) Intracellular mGluR5 can mediate synaptic plasticity in the hippocampus. $J$ Neurosci 34:4589-4598.

Quiroz JA, Tamburri P, Deptula D, Banken L, Beyer U, Rabbia M, Parkar N, Fontoura P, and Santarelli L (2016) Efficacy and safety of basimglurant as adjunctive therapy for major depression: a randomized clinical trial. JAMA Psychiatry 73:675-684.

Rahman S and Neuman RS (1996) Characterization of metabotropic glutamate receptor-mediated facilitation of N-methyl-D-aspartate depolarization of neocortical neurones. Br J Pharmacol 117:675-683.

Raka F, Di Sebastiano AR, Kulhawy SC, Ribeiro FM, Godin CM, Caetano FA, Angers $\mathrm{S}$, and Ferguson SS (2015) $\mathrm{Ca}(2+) /$ calmodulin-dependent protein kinase II interacts with group I metabotropic glutamate and facilitates receptor endocytosis and ERK1/2 signaling: role of $\beta$-amyloid. Mol Brain 8:21.

Rao AM, Hatcher JF, and Dempsey RJ (2000) Neuroprotection by group I metabotropic glutamate receptor antagonists in forebrain ischemia of gerbil. Neurosci Lett 293:1-4.

Reilmann R, Rouzade-Dominguez ML, Saft C, Süssmuth SD, Priller J, Rosser A, Rickards H, Schöls L, Pezous N, Gasparini F, et al. (2015) A randomized, placebocontrolled trial of AFQ056 for the treatment of chorea in Huntington's disease. Mov Disord 30:427-431.

Ribeiro FM, Paquet M, Ferreira LT, Cregan T, Swan P, Cregan SP, and Ferguson SS (2010) Metabotropic glutamate receptor-mediated cell signaling pathways are altered in a mouse model of Huntington's disease. J Neurosci 30:316-324

Ribeiro FM, Vieira LB, Pires RG, Olmo RP, and Ferguson SS (2017) Metabotropic glutamate receptors and neurodegenerative diseases. Pharmacol Res 115:179-191. Ritter-Makinson SL, Paquet M, Bogenpohl JW, Rodin RE, Chris Yun C, Weinman EJ, Smith Y, and Hall RA (2017) Group II metabotropic glutamate receptor interactions with NHERF scaffold proteins: implications for receptor localization in brain. Neuroscience 353:58-75.

Robbe D, Kopf M, Remaury A, Bockaert J, and Manzoni OJ (2002) Endogenous cannabinoids mediate long-term synaptic depression in the nucleus accumbens. Proc Natl Acad Sci USA 99:8384-8388. 
Roche D, Gil D, and Giraldo J (2014) Mathematical modeling of G protein-coupled receptor function: what can we learn from empirical and mechanistic models? Adv Exp Med Biol 796:159-181.

Roche KW, Tu JC, Petralia RS, Xiao B, Wenthold RJ, and Worley PF (1999) Homer $1 \mathrm{~b}$ regulates the trafficking of group I metabotropic glutamate receptors. $\mathrm{J} \mathrm{Biol}$ Chem 274:25953-25957.

Rodd ZA, McKinzie DL, Bell RL, McQueen VK, Murphy JM, Schoepp DD, and McBride WJ (2006) The metabotropic glutamate 2/3 receptor agonist LY404039 reduces alcohol-seeking but not alcohol self-administration in alcoholpreferring (P) rats. Behav Brain Res 171:207-215.

Rodrigues RJ, Alfaro TM, Rebola N, Oliveira CR, and Cunha RA (2005) Colocalization and functional interaction between adenosine $\mathrm{A}(2 \mathrm{~A})$ and metabotropic group 5 receptors in glutamatergic nerve terminals of the rat striatum. J Neurochem 92:433-441.

Rodriguez AL, Grier MD, Jones CK, Herman EJ, Kane AS, Smith RL, Williams R, Zhou Y, Marlo JE, Days EL, et al. (2010) Discovery of novel allosteric modulators of metabotropic glutamate receptor subtype 5 reveals chemical and functional diversity and in vivo activity in rat behavioral models of anxiolytic and antipsychotic activity. Mol Pharmacol 78:1105-1123.

Rodriguez AL, Nong Y, Sekaran NK, Alagille D, Tamagnan GD, and Conn PJ (2005) A close structural analog of 2-methyl-6-(phenylethynyl)-pyridine acts as a neutral allosteric site ligand on metabotropic glutamate receptor subtype 5 and blocks the effects of multiple allosteric modulators. Mol Pharmacol 68:1793-1802.

Rook JM, Noetzel MJ, Pouliot WA, Bridges TM, Vinson PN, Cho HP, Zhou Y, Gogliotti RD, Manka JT, Gregory KJ, et al. (2013) Unique signaling profiles of positive allosteric modulators of metabotropic glutamate receptor subtype 5 determine differences in in vivo activity. Biol Psychiatry 73:501-509.

Rook JM, Tantawy MN, Ansari MS, Felts AS, Stauffer SR, Emmitte KA, Kessler RM Niswender CM, Daniels JS, Jones CK, et al. (2015a) Relationship between in vivo receptor occupancy and efficacy of metabotropic glutamate receptor subtype 5 allosteric modulators with different in vitro binding profiles. Neuropsychopharmacology 40:755-765

Rook JM, Xiang Z, Lv X, Ghoshal A, Dickerson JW, Bridges TM, Johnson KA, Foster DJ, Gregory KJ, Vinson PN, et al. (2015b) Biased mGlu5-positive allosteric modulators provide in vivo efficacy without potentiating mGlu5 modulation of NMDAR currents. Neuron 86:1029-1040.

Rorick-Kehn LM, Johnson BG, Burkey JL, Wright RA, Calligaro DO, Marek GJ Nisenbaum ES, Catlow JT, Kingston AE, Giera DD, et al. (2007a) Pharmacological and pharmacokinetic properties of a structurally novel, potent, and selective metabotropic glutamate $2 / 3$ receptor agonist: in vitro characterization of agonist (-)-(1R,4S,5S,6S)-4-amino-2-sulfonylbicyclo[3.1.0]-hexane-4,6-dicarboxylic acid (LY404039). J Pharmacol Exp Ther 321:308-317.

Rorick-Kehn LM, Johnson BG, Knitowski KM, Salhoff CR, Witkin JM, Perry KW, Griffey KI, Tizzano JP, Monn JA, McKinzie DL, et al. (2007b) In vivo pharmacological characterization of the structurally novel, potent, selective mGlu2/3 receptor agonist LY404039 in animal models of psychiatric disorders. Psychopharmacology (Berl) 193:121-136.

Rorick-Kehn LM, Perkins EJ, Knitowski KM, Hart JC, Johnson BG, Schoepp DD, and McKinzie DL (2006) Improved bioavailability of the mGlu2/3 receptor agonist LY354740 using a prodrug strategy: in vivo pharmacology of LY544344. J Phar macol Exp Ther 316:905-913.

Rossi F, Marabese I, De Chiaro M, Boccella S, Luongo L, Guida F, De Gregorio D, Giordano C, de Novellis V, Palazzo E, et al. (2014) Dorsal striatum metabotropic glutamate receptor 8 affects nocifensive responses and rostral ventromedial medulla cell activity in neuropathic pain conditions. J Neurophysiol 111:2196-2209.

Rouzade-Dominguez ML, Pezous N, David OJ, Tutuian R, Bruley des Varannes S, Tack J, Malfertheiner P, Allescher HD, Ufer M, and Rühl A (2017) The selective metabotropic glutamate receptor 5 antagonist mavoglurant (AFQ056) reduces the incidence of reflux episodes in dogs and patients with moderate to severe gastroesophageal reflux disease. Neurogastroenterol Motil 29 Available from: 10.1111/ nmo.13058

Rovira X, Malhaire F, Scholler P, Rodrigo J, Gonzalez-Bulnes P, Llebaria A, Pin JP, Giraldo J, and Goudet C (2015) Overlapping binding sites drive allosteric agonism and positive cooperativity in type 4 metabotropic glutamate receptors. FASEB $J$ 29:116-130.

Rovira X, Trapero A, Pittolo S, Zussy C, Faucherre A, Jopling C, Giraldo J, Pin JP, Gorostiza P, Goudet C, et al. (2016) OptoGluNAM4.1, a photoswitchable allosteric antagonist for real-time control of mGlu4 aeceptor activity. Cell Chem Biol 23:929-934

Rowe BA, Schaffhauser H, Morales S, Lubbers LS, Bonnefous C, Kamenecka TM, McQuiston J, and Daggett LP (2008) Transposition of three amino acids transforms the human metabotropic glutamate receptor (mGluR)-3-positive allosteric modulation site to mGluR2, and additional characterization of the mGluR2-positive allosteric modulation site. J Pharmacol Exp Ther 326:240-251.

Rutrick D, Stein DJ, Subramanian G, Smith B, Fava M, Hasler G, Cha JH, Gasparin F, Donchev T, Ocwieja M, et al. (2017) Mavoglurant augmentation in OCD patients resistant to selective serotonin reuptake inhibitors: a proof-of-concept, randomized, placebo-controlled, phase 2 study. Adv Ther 34:524-541.

Saini SM, Mancuso SG, Mostaid MS, Liu C, Pantelis C, Everall IP, and Bousman CA (2017) Meta-analysis supports GWAS-implicated link between GRM3 and schizophrenia risk. Transl Psychiatry 7:e1196.

Salih H, Anghelescu I, Kezic I, Sinha V, Hoeben E, Van Nueten L, De Smedt H, and De Boer P (2015) Pharmacokinetic and pharmacodynamic characterisation of JNJ-40411813, a positive allosteric modulator of mGluR2, in two randomised, double-blind phase-I studies. $J$ Psychopharmacol 29:414-425.

Sallese M, Salvatore L, D’Urbano E, Sala G, Storto M, Launey T, Nicoletti F, Knöpfel T, and De Blasi A (2000) The G-protein-coupled receptor kinase GRK4 mediates homologous desensitization of metabotropic glutamate receptor 1 . FASEB $J \mathbf{1 4}$ : $2569-2580$.

Salling MC, Faccidomo S, and Hodge CW (2008) Nonselective suppression of operant ethanol and sucrose self-administration by the mGluR7 positive allosteric modulator AMN082. Pharmacol Biochem Behav 91:14-20.
Salt TE and Eaton SA (1995) Distinct presynaptic metabotropic receptors for L-AP4 and CCG1 on GABAergic terminals: pharmacological evidence using novel alphamethyl derivative mGluR antagonists, MAP4 and MCCG, in the rat thalamus in vivo. Neuroscience 65:5-13.

Sanders SJ, Murtha MT, Gupta AR, Murdoch JD, Raubeson MJ, Willsey AJ, ErcanSencicek AG, DiLullo NM, Parikshak NN, Stein JL, et al. (2012) De novo mutations revealed by whole-exome sequencing are strongly associated with autism. Nature 485:237-241.

Sansig G, Bushell TJ, Clarke VR, Rozov A, Burnashev N, Portet C, Gasparini F, Schmutz M, Klebs K, Shigemoto R, et al. (2001) Increased seizure susceptibility in mice lacking metabotropic glutamate receptor 7. J Neurosci 21:8734-8745.

Sartorius LJ, Nagappan G, Lipska BK, Lu B, Sei Y, Ren-Patterson R, Li Z, Weinberger DR, and Harrison PJ (2006) Alternative splicing of human metabotropic glutamate receptor 3. J Neurochem 96:1139-1148.

Satow A, Suzuki G, Maehara S, Hikichi H, Murai T, Murai T, Kawagoe-Takaki H, Hata M, Ito S, Ozaki S, et al. (2009) Unique antipsychotic activities of the selective metabotropic glutamate receptor 1 allosteric antagonist 2-cyclopropyl-5-[1-(2-fluoro-3-pyridinyl)-5-methyl-1H-1,2,3-triazol-4-yl]-2,3-dihydro-1H-isoindol-1-one. $J$ Pharmacol Exp Ther 330:179-190.

Saugstad JA, Kinzie JM, Mulvihill ER, Segerson TP, and Westbrook GL (1994) Cloning and expression of a new member of the L-2-amino-4-phosphonobutyric acid-sensitive class of metabotropic glutamate receptors. Mol Pharmacol 45: 367-372

Saugstad JA, Kinzie JM, Shinohara MM, Segerson TP, and Westbrook GL (1997) Cloning and expression of rat metabotropic glutamate receptor 8 reveals a distinct pharmacological profile. Mol Pharmacol 51:119-125.

Saugstad JA, Segerson TP, and Westbrook GL (1996) Metabotropic glutamate receptors activate G-protein-coupled inwardly rectifying potassium channels in Xenopus oocytes. J Neurosci 16:5979-5985.

Saunders R, Nahorski SR, and Challiss RA (1998) A modulatory effect of extracellular $\mathrm{Ca} 2+$ on type 1alpha metabotropic glutamate receptor-mediated signalling. Neuropharmacology 37:273-276.

Scandroglio P, Brusa R, Lozza G, Mancini I, Petrò R, Reggiani A, and Beltramo M (2010) Evaluation of cannabinoid receptor 2 and metabotropic glutamate receptor 1 functional responses using a cell impedance-based technology. J Biomol Screen 15: 1238-1247.

Schaffhauser H, Cai Z, Hubalek F, Macek TA, Pohl J, Murphy TJ, and Conn PJ (2000) cAMP-dependent protein kinase inhibits mGluR2 coupling to G-proteins by direct receptor phosphorylation. J Neurosci 20:5663-5670.

Schaffhauser H, Knoflach F, Pink JR, Bleuel Z, Cartmell J, Goepfert F, Kemp JA, Richards JG, Adam G, and Mutel V (1998) Multiple pathways for regulation of the $\mathrm{KCl}$-induced $[3 \mathrm{H}]-\mathrm{GABA}$ release by metabotropic glutamate receptors, in primary rat cortical cultures. Brain Res 782.91-104.

Schaffhauser H, Rowe BA, Morales S, Chavez-Noriega LE, Yin R, Jachec C, Rao SP, Bain G, Pinkerton AB, Vernier JM, et al. (2003) Pharmacological characterization and identification of amino acids involved in the positive modulation of metabotropic glutamate receptor subtype 2. Mol Pharmacol 64:798-810.

Scherer SW, Soder S, Duvoisin RM, Huizenga JJ, and Tsui LC (1997) The human metabotropic glutamate receptor 8 (GRM8) gene: a disproportionately large gene located at 7q31.3-q32.1. Genomics 44:232-236.

Schkeryantz JM, Kingston AE, and Johnson MP (2007) Prospects for metabotropic glutamate 1 receptor antagonists in the treatment of neuropathic pain. $J \mathrm{Med}$ Chem 50:2563-2568.

Schoepp DD (2001) Unveiling the functions of presynaptic metabotropic glutamate receptors in the central nervous system. J Pharmacol Exp Ther 299:12-20.

Schoepp DD, Goldsworthy J, Johnson BG, Salhoff CR, and Baker SR (1994) 3,5-dihydroxyphenylglycine is a highly selective agonist for phosphoinositide-linked metabotropic glutamate receptors in the rat hippocampus. J Neurochem 63:769-772

Schoepp DD, Johnson BG, Wright RA, Salhoff CR, Mayne NG, Wu S, Cockerman SL, Burnett JP, Belegaje R, Bleakman D, et al. (1997) LY354740 is a potent and highly selective group II metabotropic glutamate receptor agonist in cells expressing human glutamate receptors. Neuropharmacology 36:1-11.

Schoepp DD, Wright RA, Levine LR, Gaydos B, and Potter WZ (2003) LY354740, an mGlu2/3 receptor agonist as a novel approach to treat anxiety/stress. Stress 6:189-197.

Scholler P, Nevoltris D, de Bundel D, Bossi S, Moreno-Delgado D, Rovira X, Møller TC, El Moustaine D, Mathieu M, Blanc E, et al. (2017) Allosteric nanobodies uncover a role of hippocampal mGlu2 receptor homodimers in contextual fear consolidation. Nat Commun 8:1967.

Schreiber R, Lowe D, Voerste A, and De Vry J (2000) LY354740 affects startle responding but not sensorimotor gating or discriminative effects of phencyclidine. Eur $J$ Pharmacol 388:R3-R4.

Schröder UH, Müller T, Schreiber R, Stolle A, Zuschratter W, Balschun D, Jork R, and Reymann KG (2008) The potent non-competitive mGlu1 receptor antagonist BAY 36-7620 differentially affects synaptic plasticity in area cornu ammonis 1 of rat hippocampal slices and impairs acquisition in the water maze task in mice. Neuroscience 157:385-395.

Schroeder JP, Overstreet DH, and Hodge CW (2005) The mGluR5 antagonist MPEP decreases operant ethanol self-administration during maintenance and after repeated alcohol deprivations in alcohol-preferring $(\mathrm{P})$ rats. Psychopharmacology (Berl) 179:262-270.

Schulz HL, Stohr H, and Weber BH (2002) Characterization of three novel isoforms of the metabotrobic glutamate receptor 7 (GRM7). Neurosci Lett 326:37-40.

Sebastianutto I, Goyet E, Andreoli L, Font-Ingles J, Moreno-Delgado D, Bouquier N, Jahannault-Talignani C, Moutin E, Di Menna L, Maslava N, et al. (2020) D1mGlu5 heteromers mediate noncanonical dopamine signaling in Parkinson's disease. J Clin Invest 130:1168-1184

Seebahn A, Rose M, and Enz R (2008) RanBPM is expressed in synaptic layers of the mammalian retina and binds to metabotropic glutamate receptors. FEBS Lett $\mathbf{5 8 2}$ $2453-2457$.

Seeman P (2013) An agonist at glutamate and dopamine D2 receptors, LY404039. Neuropharmacology 66:87-88. 
Sekiyama N, Hayashi Y, Nakanishi S, Jane DE, Tse HW, Birse EF, and Watkins JC (1996) Structure-activity relationships of new agonists and antagonists of different metabotropic glutamate receptor subtypes. Br J Pharmacol 117:1493-1503.

Selvam C, Goudet C, Oueslati N, Pin JP, and Acher FC (2007) L-(+)-2-Amino-4thiophosphonobutyric acid (L-thioAP4), a new potent agonist of group III metabotropic glutamate receptors: increased distal acidity affords enhanced potency. $J$ Med Chem 50:4656-4664.

Selvam C, Lemasson IA, Brabet I, Oueslati N, Karaman B, Cabaye A, Tora AS, Commare B, Courtiol T, Cesarini S, et al. (2018) Increased potency and selectivity for group III metabotropic glutamate receptor agonists binding at dual sites. J Med Chem 61:1969-1989.

Sengmany K, Hellyer SD, Albold S, Wang T, Conn PJ, May LT, Christopoulos A Leach K, and Gregory KJ (2019) Kinetic and system bias as drivers of metabotropic glutamate receptor 5 allosteric modulator pharmacology. Neuropharmacology 149 83-96.

Sengmany K, Singh J, Stewart GD, Conn PJ, Christopoulos A, and Gregory KJ (2017) Biased allosteric agonism and modulation of metabotropic glutamate receptor 5: implications for optimizing preclinical neuroscience drug discovery. Neurophar macology 115:60-72.

Sengmany K, Hellyer SD, Christopoulos A, Lapinsky DJ, Leach K, and Gregory KJ (2020) Differential contribution of metabotropic glutamate receptor 5 common allosteric binding site residues to biased allosteric agonism. Biochem Pharmacol 177: 114011.

Sergeeva OA, Doreulee N, Chepkova AN, Kazmierczak T, and Haas HL (2007) Longterm depression of cortico-striatal synaptic transmission by DHPG depends on endocannabinoid release and nitric oxide synthesis. Eur J Neurosci 26:1889-1894.

Servitja JM, Masgrau R, Pardo R, Sarri E, von Eichel-Streiber C, Gutkind JS and Picatoste F (2003) Metabotropic glutamate receptors activate phospholipase D in astrocytes through a protein kinase C-dependent and Rho-independent pathway. Neuropharmacology 44:171-180.

Servitja JM, Masgrau R, Sarri E, and Picatoste F (1999) Group I metabotropic glutamate receptors mediate phospholipase $\mathrm{D}$ stimulation in rat cultured astrocytes. $J$ Neurochem 72:1441-1447.

Shah UH and González-Maeso J (2019) Serotonin and glutamate interactions in preclinical schizophrenia models. ACS Chem Neurosci 10:3068-3077.

Shannon HE, Peters SC, and Kingston AE (2005) Anticonvulsant effects of LY456236, a selective mGlu1 receptor antagonist. Neuropharmacology 49 (Supp 1):188-195.

Sharko AC and Hodge CW (2008) Differential modulation of ethanol-induced sedation and hypnosis by metabotropic glutamate receptor antagonists in C57BL/6J mice. Alcohol Clin Exp Res 32:67-76.

Sharon D, Vorobiov D, and Dascal N (1997) Positive and negative coupling of the metabotropic glutamate receptors to a $\mathrm{G}$ protein-activated $\mathrm{K}+$ channel, GIRK, in Xenopus oocytes. J Gen Physiol 109:477-490.

Sheffler DJ and Conn PJ (2008) Allosteric potentiators of metabotropic glutamate receptor subtype 1a differentially modulate independent signaling pathways in baby hamster kidney cells. Neuropharmacology 55:419-427.

Sheffler DJ, Wenthur CJ, Bruner JA, Carrington SJS, Vinson PN, Gogi KK, Blobaum AL, Morrison RD, Vamos M, Cosford NDP, et al. (2012) Development of a novel, CNS-penetrant, metabotropic glutamate receptor 3 (mGlu3) NAM probe (ML289) derived from a closely related mGlu5 PAM. Bioorg Med Chem Lett 22:3921-3925.

Shen Y, Rampino MA, Carroll RC, and Nawy S (2012) G-protein-mediated inhibition of the Trp channel TRPM1 requires the G $\beta \gamma$ dimer. Proc Natl Acad Sci USA 109 $8752-8757$

Shigemoto R, Kinoshita A, Wada E, Nomura S, Ohishi H, Takada M, Flor PJ, Neki A, Abe T, Nakanishi S, et al. (1997) Differential presynaptic localization of metabotropic glutamate receptor subtypes in the rat hippocampus. $J$ Neurosci 17:7503-7522.

Shinozaki H and Shibuya I (1974) A new potent excitant, quisqualic acid: effects on crayfish neuromuscular junction. Neuropharmacology 13:665-672.

Silverman JL, Tolu SS, Barkan CL, and Crawley JN (2010) Repetitive self-grooming behavior in the BTBR mouse model of autism is blocked by the mGluR5 antagonist MPEP. Neuropsychopharmacology 35:976-989.

Silverstein FS, Chen R, and Johnston MV (1986) The glutamate analogue quisqualic acid is neurotoxic in striatum and hippocampus of immature rat brain. Neurosci Lett 71:13-18.

Slack RJ and Hall DA (2012) Development of operational models of receptor activation including constitutive receptor activity and their use to determine the efficacy of the chemokine CCL17 at the CC chemokine receptor CCR4. Br J Pharmacol 166:1774-1792

Sławińska A, Wierońska JM, Stachowicz K, Marciniak M, Lasoń-Tyburkiewicz M, Gruca P, Papp M, Kusek M, Tokarski K, Doller D, et al. (2013) The antipsychoticlike effects of positive allosteric modulators of metabotropic glutamate mGlu4 receptors in rodents. Br J Pharmacol 169:1824-1839

Smith FL, Smith PA, Dewey WL, and Javed RR (2004) Effects of mGlu1 and mGlu5 metabotropic glutamate antagonists to reverse morphine tolerance in mice. Eur $J$ Pharmacol 492:137-142.

Snyder EM, Philpot BD, Huber KM, Dong X, Fallon JR, and Bear MF (2001) Internalization of ionotropic glutamate receptors in response to mGluR activation. Nat Neurosci 4:1079-1085.

Soloviev MM, Ciruela F, Chan WY, and McIlhinney RA (1999) Identification, cloning and analysis of expression of a new alternatively spliced form of the metabotropic glutamate receptor mGluR1 mRNA1. Biochim Biophys Acta 1446:161-166.

Song Z, He CD, Liu J, Sun C, Lu P, Li L, Gao L, Zhang Y, Xu Y, Shan L, et al. (2012) Blocking glutamate-mediated signalling inhibits human melanoma growth and migration. Exp Dermatol 21:926-931.

Spampinato SF, Copani A, Nicoletti F, Sortino MA, and Caraci F (2018) Metabotropic glutamate receptors in glial cells: a new potential target for neuroprotection? Front Mol Neurosci 11:414.

Stachowicz K, Chojnacka-Wójcik E, Kłak K, and Pilc A (2006) Anxiolytic-like effects of group III mGlu receptor ligands in the hippocampus involve GABAA signaling. Pharmacol Rep 58:820-826.
Stachowicz K, Kłak K, Kłodzińska A, Chojnacka-Wojcik E, and Pilc A (2004) Anxiolytic-like effects of PHCCC, an allosteric modulator of mGlu4 receptors, in rats. Eur J Pharmacol 498:153-156.

Steckler T, Lavreysen H, Oliveira AM, Aerts N, Van Craenendonck H, Prickaerts J, Megens A, and Lesage AS (2005a) Effects of mGlu1 receptor blockade on anxietyrelated behaviour in the rat lick suppression test. Psychopharmacology (Berl) 179: 198-206.

Steckler T, Oliveira AF, Van Dyck C, Van Craenendonck H, Mateus AM, Langlois X, Lesage AS, and Prickaerts J (2005b) Metabotropic glutamate receptor 1 blockade impairs acquisition and retention in a spatial Water maze task. Behav Brain Res 164:52-60.

Stocchi F, Rascol O, Destee A, Hattori N, Hauser RA, Lang AE, Poewe W, Stacy M, Tolosa E, Gao H, et al. (2013) AFQ056 in Parkinson patients with levodopainduced dyskinesia: 13-week, randomized, dose-finding study. Mov Disord 28 $1838-1846$

Sugiyama H, Ito I, and Hirono C (1987) A new type of glutamate receptor linked to inositol phospholipid metabolism. Nature 325:531-533.

Suh YH, Park JY, Park S, Jou I, Roche PA, and Roche KW (2013) Regulation of metabotropic glutamate receptor 7 (mGluR7) internalization and surface expression by Ser/Thr protein phosphatase 1. J Biol Chem 288:17544-17551.

Sukoff Rizzo SJ, Leonard SK, Gilbert A, Dollings P, Smith DL, Zhang MY, Di L, Platt BJ, Neal S, Dwyer JM, et al. (2011) The metabotropic glutamate receptor 7 allosteric modulator AMN082: a monoaminergic agent in disguise? J Pharmacol Exp Ther 338:345-352.

Suzuki G, Kawagoe-Takaki H, Inoue T, Kimura T, Hikichi H, Murai T, Satow A, Hata M, Maehara S, Ito S, et al. (2009) Correlation of receptor occupancy of metabotropic glutamate receptor subtype 1 (mGluR1) in mouse brain with in vivo activity of allosteric mGluR1 antagonists. J Pharmacol Sci 110:315-325.

Suzuki G, Kimura T, Satow A, Kaneko N, Fukuda J, Hikichi H, Sakai N, Maehara S, Kawagoe-Takaki H, Hata M, et al. (2007a) Pharmacological characterization of a new, orally active and potent allosteric metabotropic glutamate receptor 1 antagonist, 4-[1-(2-fluoropyridin-3-yl)-5-methyl-1H-1,2,3-triazol-4-yl]-N-isopropyl-Nmethyl-3,6-dihydropyridine-1(2H)-carboxamide (FTIDC). J Pharmacol Exp Ther 321:1144-1153.

Suzuki G, Satow A, and Ohta H (2010) Effect of CFMTI, an allosteric metabotropic glutamate receptor 1 antagonist with antipsychotic activity, on Fos expression in regions of the brain related to schizophrenia. Neuroscience 168:787-796.

Suzuki G, Tsukamoto N, Fushiki H, Kawagishi A, Nakamura M, Kurihara H, Mitsuya $\mathrm{M}$, Ohkubo $\mathrm{M}$, and Ohta $\mathrm{H}$ (2007b) In vitro pharmacological characterization of novel isoxazolopyridone derivatives as allosteric metabotropic glutamate receptor 7 antagonists. J Pharmacol Exp Ther 323:147-156.

Swedberg MD, Ellgren M, and Raboisson P (2014) mGluR5 antagonist-induced psychoactive properties: MTEP drug discrimination, a pharmacologically selective non-NMDA effect with apparent lack of reinforcing properties. J Pharmacol Exp Ther 349:155-164.

Swedberg MD and Raboisson P (2014) AZD9272 and AZD2066: selective and highly central nervous system penetrant mGluR5 antagonists characterized by their discriminative effects. $J$ Pharmacol Exp Ther 350:212-222.

Szczurowska E and Mareš P (2012) Positive allosteric modulator of mGluR4 PHCCC exhibits proconvulsant action in three models of epileptic seizures in immature rats. Physiol Res 61:619-628.

Tanabe Y, Masu M, Ishii T, Shigemoto R, and Nakanishi S (1992) A family of metabotropic glutamate receptors. Neuron 8:169-179.

Tassin V, Girard B, Chotte A, Fontanaud P, Rigault D, Kalinichev M, Perroy J, Acher F, Fagni L, and Bertaso F (2016) Phasic and Tonic mGlu7 receptor activity modulates the thalamocortical network. Front Neural Circuits 10:31.

Tatarczyńska E, Kłodzińska A, Kroczka B, Chojnacka-Wójcik E, and Pilc A (2001) The antianxiety-like effects of antagonists of group I and agonists of group II and III metabotropic glutamate receptors after intrahippocampal administration. Psychopharmacology (Berl) 158:94-99.

Tatarczyńska E, Pałucha A, Szewczyk B, Chojnacka-Wójcik E, Wierońska J, and Pilc A (2002) Anxiolytic- and antidepressant-like effects of group III metabotropic glutamate agonist (1S,3R,4S)-1-aminocyclopentane-1,3,4-tricarboxylic acid (ACPTI) in rats. Pol J Pharmacol 54:707-710.

Tateyama M and Kubo Y (2008) Regulatory role of C-terminus in the G-protein coupling of the metabotropic glutamate receptor 1 . J Neurochem 107:1036-1046.

Techlovská Š, Chambers JN, Dvořáková M, Petralia RS, Wang YX, Hájková A, Nová A, Franková D, Prezeau L, and Blahos J (2014) Metabotropic glutamate receptor 1 splice variants mGluR1a and mGluR1b combine in mGluR1a/b dimers in vivo. Neuropharmacology 86:329-336.

Testa CM, Standaert DG, Young AB, and Penney JB Jr. (1994) Metabotropic glutamate receptor mRNA expression in the basal ganglia of the rat. J Neurosci 14 3005-3018.

Thomas NK, Jane DE, Tse HW, and Watkins JC (1996) alpha-Methyl derivatives of serine-O-phosphate as novel, selective competitive metabotropic glutamate receptor antagonists. Neuropharmacology 35:637-642.

Thomsen C (1997) The L-AP4 receptor. Gen Pharmacol 29:151-158.

Thomsen C and Dalby NO (1998) Roles of metabotropic glutamate receptor subtypes in modulation of pentylenetetrazole-induced seizure activity in mice. Neuropharmacology 37:1465-1473.

Thomsen C, Kristensen P, Mulvihill E, Haldeman B, and Suzdak PD (1992) L-2amino-4-phosphonobutyrate (L-AP4) is an agonist at the type IV metabotropic glutamate receptor which is negatively coupled to adenylate cyclase. Eur J Pharmacol 227:361-362.

Thomsen C, Pekhletski R, Haldeman B, Gilbert TA, O'Hara P, and Hampson DR (1997) Cloning and characterization of a metabotropic glutamate receptor mGluR4b. Neuropharmacology 36:21-30.

Thomsen C and Suzdak PD (1993) Serine-O-phosphate has affinity for type IV, but not type I, metabotropic glutamate receptor. Neuroreport 4:1099-1101.

Tison F, Keywood C, Wakefield M, Durif F, Corvol JC, Eggert K, Lew M, Isaacson S, Bezard E, Poli SM, et al. (2016) A phase 2A trial of the novel mGluR5-negative 
allosteric modulator dipraglurant for levodopa-induced dyskinesia in Parkinson's disease. Mov Disord 31:1373-1380.

Tizzano JP, Griffey KI, and Schoepp DD (1995) Induction or protection of limbic seizures in mice by mGluR subtype selective agonists. Neuropharmacology 34: 1063-1067.

Tizzano JP, Griffey KI, and Schoepp DD (2002) The anxiolytic action of mGlu2/3 receptor agonist, LY354740, in the fear-potentiated startle model in rats is mechanistically distinct from diazepam. Pharmacol Biochem Behav 73:367-374.

Tomita N, Murata M, Watanabe H, Ichikawa T, Washiyama K, Kumanishi T, and Takahashi Y (2000) The effects of DCG-IV and L-CCG-1 upon phencyclidine (PCP)induced locomotion and behavioral changes in mice. Ann N Y Acad Sci 914:284-291.

Toms NJ, Jane DE, Kemp MC, Bedingfield JS, and Roberts PJ (1996) The effects of (RS)-alpha-cyclopropyl-4-phosphonophenylglycine ((RS)-CPPG), a potent and selective metabotropic glutamate receptor antagonist. Br J Pharmacol 119:851-854

Tora AS, Rovira X, Cao AM, Cabayé A, Olofsson L, Malhaire F, Scholler P, Baik H, Van Eeckhaut A, Smolders I, et al. (2018) Chloride ions stabilize the glutamateinduced active state of the metabotropic glutamate receptor 3. Neuropharmacology 140:275-286.

Tora AS, Rovira X, Dione I, Bertrand HO, Brabet I, De Koninck Y, Doyon N, Pin JP, Acher F, and Goudet C (2015) Allosteric modulation of metabotropic glutamate receptors by chloride ions. FASEB J 29:4174-4188.

Trenkwalder C, Stocchi F, Poewe W, Dronamraju N, Kenney C, Shah A, von Raison F, and Graf A (2016) Mavoglurant in Parkinson's patients with l-Dopa-induced dyskinesias: Two randomized phase 2 studies. Mov Disord 31:1054-1058.

Tresadern G, Trabanco AA, Pérez-Benito L, Overington JP, van Vlijmen HWT, and van Westen GJP (2017) Identification of allosteric modulators of metabotropic glutamate 7 receptor using proteochemometric modeling. J Chem Inf Model 57:2976-2985.

Trombley PQ and Westbrook GL (1992) L-AP4 inhibits calcium currents and synaptic transmission via a G-protein-coupled glutamate receptor. $J$ Neurosci 12 2043-2050.

Tsuchiya D, Kunishima N, Kamiya N, Jingami H, and Morikawa K (2002) Structural views of the ligand-binding cores of a metabotropic glutamate receptor complexed with an antagonist and both glutamate and Gd3+. Proc Natl Acad Sci USA 99: $2660-2665$.

Tu JC, Xiao B, Naisbitt S, Yuan JP, Petralia RS, Brakeman P, Doan A, Aakalu VK Lanahan AA, Sheng M, et al. (1999) Coupling of mGluR/Homer and PSD-95 complexes by the Shank family of postsynaptic density proteins. Neuron 23 $583-592$

Turner BD, Rook JM, Lindsley CW, Conn PJ, and Grueter BA (2018) mGlu and $_{1}$ $\mathrm{mGlu}_{5}$ modulate distinct excitatory inputs to the nucleus accumbens shell. $\mathrm{Neu}$ ropsychopharmacology 43:2075-2082.

Tyszkiewicz JP, Gu Z, Wang X, Cai X, and Yan Z (2004) Group II metabotropic glutamate receptors enhance NMDA receptor currents via a protein kinase C-dependent mechanism in pyramidal neurones of rat prefrontal cortex. J Physiol 554:765-777.

Uematsu K, Heiman M, Zelenina M, Padovan J, Chait BT, Aperia A, Nishi A and Greengard P (2015) Protein kinase A directly phosphorylates metabotropic glutamate receptor 5 to modulate its function. $J$ Neurochem 132:677-686.

Ullmer C, Zoffmann S, Bohrmann B, Matile H, Lindemann L, Flor P, and Malherbe P (2012) Functional monoclonal antibody acts as a biased agonist by inducing in ternalization of metabotropic glutamate receptor 7. Br J Pharmacol 167:1448-1466.

Um JW, Kaufman AC, Kostylev M, Heiss JK, Stagi M, Takahashi H, Kerrisk ME Vortmeyer A, Wisniewski T, Koleske AJ, et al. (2013) Metabotropic glutamate receptor 5 is a coreceptor for Alzheimer a $\beta$ oligomer bound to cellular prion protein. Neuron 79:887-902.

Valenti O, Conn PJ, and Marino MJ (2002) Distinct physiological roles of the Gqcoupled metabotropic glutamate receptors Co-expressed in the same neuronal populations. J Cell Physiol 191:125-137.

Valerio A, Ferraboli S, Paterlini M, Spano P, and Barlati S (2001a) Identification of novel alternatively-spliced mRNA isoforms of metabotropic glutamate receptor 6 gene in rat and human retina. Gene 262:99-106.

Valerio A, Zoppi N, Ferraboli S, Paterlini M, Ferrario M, Barlati S, and Spano P (2001b) Alternative splicing of mGlu6 gene generates a truncated glutamate receptor in rat retina. Neuroreport 12:2711-2715.

van Wyk M, Pielecka-Fortuna J, Löwel S, and Kleinlogel S (2015) Restoring the ON switch in blind retinas: opto-mGluR6, a next-generation, cell-tailored optogenetic tool. PLoS Biol 13:e1002143.

Vardi N, Duvoisin R, Wu G, and Sterling P (2000) Localization of mGluR6 to dendrites of ON bipolar cells in primate retina. J Comp Neurol 423:402-412.

Varnäs K, Cselényi Z, Arakawa R, Nag S, Stepanov V, Moein MM, Johnström P, Kingston L, Elmore CS, Halldin C, et al. (2020) The pro-psychotic metabotropic glutamate receptor compounds fenobam and AZD9272 share binding sites with monoamine oxidase-B inhibitors in humans. Neuropharmacology 162:107809.

Varty GB, Grilli M, Forlani A, Fredduzzi S, Grzelak ME, Guthrie DH, Hodgson RA, Lu SX, Nicolussi E, Pond AJ, et al. (2005) The antinociceptive and anxiolytic-like effects of the metabotropic glutamate receptor 5 (mGluR5) antagonists, MPEP and MTEP, and the mGluR1 antagonist, LY456236, in rodents: a comparison of efficacy and side-effect profiles. Psychopharmacology (Berl) 179:207-217.

Vergouts M, Doyen PJ, Peeters M, Opsomer R, Michiels T, and Hermans E (2017) PKC epsilon-dependent calcium oscillations associated with metabotropic glutamate receptor 5 prevent agonist-mediated receptor desensitization in astrocytes. $J$ Neurochem 141:387-399.

Verma A and Moghaddam B (1998) Regulation of striatal dopamine release by metabotropic glutamate receptors. Synapse 28:220-226.

Vernon AC, Zbarsky V, Datla KP, Dexter DT, and Croucher MJ (2007) Selective activation of group III metabotropic glutamate receptors by L-(+)-2-amino-4phosphonobutryic acid protects the nigrostriatal system against 6hydroxydopamine toxicity in vivo. J Pharmacol Exp Ther 320:397-409.

Vilar B, Busserolles J, Ling B, Laffray S, Ulmann L, Malhaire F, Chapuy E, Aissouni Y, Etienne M, Bourinet E, et al. (2013) Alleviating pain hypersensitivity through activation of type 4 metabotropic glutamate receptor. J Neurosci 33:18951-18965.
Vinueza Veloz MF, Buijsen RA, Willemsen R, Cupido A, Bosman LW, Koekkoek SK, Potters JW, Oostra BA, and De Zeeuw CI (2012) The effect of an mGluR5 inhibitor on procedural memory and avoidance discrimination impairments in Fmr1 KO mice. Genes Brain Behav 11:325-331.

Volk LJ, Daly CA, and Huber KM (2006) Differential roles for group $1 \mathrm{mGluR}$ subtypes in induction and expression of chemically induced hippocampal long-term depression. $J$ Neurophysiol 95:2427-2438.

Volpi C, Fallarino F, Mondanelli G, Macchiarulo A, and Grohmann U (2018) Opportunities and challenges in drug discovery targeting metabotropic glutamate receptor 4. Expert Opin Drug Discov 13:411-423.

Volpi C, Mondanelli G, Pallotta MT, Vacca C, Iacono A, Gargaro M, Albini E, Bianchi R, Belladonna ML, Celanire S, et al. (2016) Allosteric modulation of metabotropic glutamate receptor 4 activates IDO1-dependent, immunoregulatory signaling in dendritic cells. Neuropharmacology 102:59-71.

Vranesic I, Ofner S, Flor PJ, Bilbe G, Bouhelal R, Enz A, Desrayaud S, McAllister K, Kuhn R, and Gasparini F (2014) AFQ056/mavoglurant, a novel clinically effective mGluR5 antagonist: identification, SAR and pharmacological characterization. Bioorg Med Chem 22:5790-5803.

Wada E, Shigemoto R, Kinoshita A, Ohishi H, and Mizuno N (1998) Metabotropic glutamate receptor subtypes in axon terminals of projection fibers from the main and accessory olfactory bulbs: a light and electron microscopic immunohistochemical study in the rat. J Comp Neurol 393:493-504.

Wagner KV, Hartmann J, Labermaier C, Häusl AS, Zhao G, Harbich D, Schmid B, Wang XD, Santarelli S, Kohl C, et al. (2015) Homer1/mGluR5 activity moderates vulnerability to chronic social stress. Neuropsychopharmacology 40:1222-1233.

Walker AG and Conn PJ (2015) Group I and group II metabotropic glutamate receptor allosteric modulators as novel potential antipsychotics. Curr Opin Pharmacol 20:40-45.

Walker AG, Wenthur CJ, Xiang Z, Rook JM, Emmitte KA, Niswender CM, Lindsley CW, and Conn PJ (2015) Metabotropic glutamate receptor 3 activation is required for long-term depression in medial prefrontal cortex and fear extinction. Proc Natl Acad Sci USA 112:1196-1201.

Wang H, Jiang W, Yang R, and Li Y (2011) Spinal metabotropic glutamate receptor 4 is involved in neuropathic pain. Neuroreport 22:244-248.

Wang H, Westin L, Nong Y, Birnbaum S, Bendor J, Brismar H, Nestler E, Aperia A, Flajolet M, and Greengard P (2009) Norbin is an endogenous regulator of metabotropic glutamate receptor 5 signaling. Science 326:1554-1557.

Watkins J and Collingridge G (1994) Phenylglycine derivatives as antagonists of metabotropic glutamate receptors. Trends Pharmacol Sci 15:333-342.

Watson LM, Bamber E, Schnekenberg RP, Williams J, Bettencourt C, Lickiss J, Jayawant S, Fawcett K, Clokie S, Wallis Y, et al. (2017) Dominant mutations in GRM1 cause spinocerebellar ataxia Type 44. Am J Hum Genet 101:451-458.

Watterson LR, Kufahl PR, Nemirovsky NE, Sewalia K, Hood LE, and Olive MF (2013) Attenuation of reinstatement of methamphetamine-, sucrose-, and foodseeking behavior in rats by fenobam, a metabotropic glutamate receptor 5 negative allosteric modulator. Psychopharmacology (Berl) 225:151-159.

Wellendorph $\mathrm{P}$ and Bräuner-Osborne $\mathrm{H}$ (2009) Molecular basis for amino acid sensing by family C G-protein-coupled receptors. $\mathrm{Br} J$ Pharmacol 156:869-884.

Wenthur CJ, Morrison RD, Daniels JS, Conn PJ, and Lindsley CW (2014) Synthesis and SAR of substituted pyrazolo[1,5-a]quinazolines as dual $\mathrm{mGlu}(2) / \mathrm{mGlu}(3)$ NAMs. Bioorg Med Chem Lett 24:2693-2698.

Westmark PR, Dekundy A, Gravius A, Danysz W, and Westmark CJ (2018) Rescue of Fmr1 $1^{\mathrm{KO}}$ phenotypes with $\mathrm{mGluR}_{5}$ inhibitors: MRZ-8456 versus AFQ-056. Neurobiol Dis 119:190-198.

White E, McKenna J, Cavanaugh A, and Breitwieser GE (2009) Pharmacochaperonemediated rescue of calcium-sensing receptor loss-of-function mutants. Mol Endocrinol 23:1115-1123.

Wierońska JM, Stachowicz K, Acher F, Lech T, and Pilc A (2012a) Opposing efficacy of group III mGlu receptor activators, LSP1-2111 and AMN082, in animal models of positive symptoms of schizophrenia. Psychopharmacology (Berl) 220: 481-494.

Wierońska JM, Stachowicz K, Brański P, Pałucha-Poniewiera A, and Pilc A (2012b) On the mechanism of anti-hyperthermic effects of LY379268 and LY487379, group II mGlu receptors activators, in the stress-induced hyperthermia in singly housed mice. Neuropharmacology 62:322-331.

Wierońska JM, Stachowicz K, Pałucha-Poniewiera A, Acher F, Brański P, and Pilc A (2010) Metabotropic glutamate receptor 4 novel agonist LSP1-2111 with anxiolytic, but not antidepressant-like activity, mediated by serotonergic and GABAergic systems. Neuropharmacology 59:627-634.

Wiśniewski K and Car H (2002) (S)-3,5-DHPG: a review. CNS Drug Rev 8:101-116.

Witkin JM, Monn JA, Schoepp DD, Li X, Overshiner C, Mitchell SN, Carter G, Johnson B, Rasmussen K, and Rorick-Kehn LM (2016) The rapidly acting antidepressant ketamine and the mGlu2/3 receptor antagonist LY341495 rapidly engage dopaminergic mood circuits. $J$ Pharmacol Exp Ther 358:71-82.

Woltering TJ, Wichmann J, Goetschi E, Adam G, Kew JN, Knoflach F, Ballard TM, Huwyler J, Mutel V, and Gatti S (2008) Synthesis and characterization of 1,3dihydro-benzo[b][1,4]diazepin-2-one derivatives: Part 3. New potent noncompetitive metabotropic glutamate receptor 2/3 antagonists. Bioorg Med Chem Lett 18:2725-2729.

Woltering TJ, Wichmann J, Goetschi E, Knoflach F, Ballard TM, Huwyler J, and Gatti S (2010) Synthesis and characterization of 1,3-dihydro-benzo[b] $[1,4]$ diazepin-2-one derivatives: Part 4. In vivo active potent and selective noncompetitive metabotropic glutamate receptor 2/3 antagonists. Bioorg Med Chem Lett 20:6969-6974.

Wood MR, Hopkins CR, Brogan JT, Conn PJ, and Lindsley CW (2011) "Molecular switches" on mGluR allosteric ligands that modulate modes of pharmacology. Biochemistry 50:2403-2410.

Woolley ML, Pemberton DJ, Bate S, Corti C, and Jones DN (2008) The mGlu2 but not the mGlu3 receptor mediates the actions of the mGluR2/3 agonist, LY379268, in mouse models predictive of antipsychotic activity. Psychopharmacology (Berl) 196: $431-440$. 
Woźniak M, Gołembiowska K, Noworyta-Sokołowska K, Acher F, Cieślik P, Kusek M, Tokarski K, Pilc A and Wierońska JM (2017) Neurochemical and behavioral studies on the $5-\mathrm{HT}_{1 \mathrm{~A}}$-dependent antipsychotic action of the $\mathrm{mGlu}_{4}$ receptor agonist LSP4-2022. Neuropharmacology 115:149-165.

Wright RA, Johnson BG, Zhang C, Salhoff C, Kingston AE, Calligaro DO, Monn JA, Schoepp DD, and Marek GJ (2013) CNS distribution of metabotropic glutamate 2 and 3 receptors: transgenic mice and $\left[{ }^{3} \mathrm{H}\right] \mathrm{LY} 459477$ autoradiography. Neuropharmacology 66:89-98.

Wroblewska B, Wegorzewska IN, Bzdega T, Olszewski RT, and Neale JH (2006) Differential negative coupling of type 3 metabotropic glutamate receptor to cyclic GMP levels in neurons and astrocytes. J Neurochem 96:1071-1077.

Wu H, Wang C, Gregory KJ, Han GW, Cho HP, Xia Y, Niswender CM, Katritch V, Meiler J, Cherezov V, et al. (2014) Structure of a class C GPCR metabotropic glutamate receptor 1 bound to an allosteric modulator. Science 344:58-64.

Wu S, Wright RA, Rockey PK, Burgett SG, Arnold JS, Rosteck PR Jr., Johnson BG, Schoepp DD, and Belagaje RM (1998) Group III human metabotropic glutamate receptors 4, 7 and 8: molecular cloning, functional expression, and comparison of pharmacological properties in RGT cells. Brain Res Mol Brain Res 53:88-97.

Xi D, Li YC, Snyder MA, Gao RY, Adelman AE, Zhang W, Shumsky JS, and Gao WJ (2011) Group II metabotropic glutamate receptor agonist ameliorates MK801induced dysfunction of NMDA receptors via the Akt/GSK-3 $\beta$ pathway in adult rat prefrontal cortex. Neuropsychopharmacology 36:1260-1274.

Xiang Z, Lv X, Maksymetz J, Stansley BJ, Ghoshal A, Gogliotti RG, Niswender CM, Lindsley CW, and Conn PJ (2019) $\mathrm{mGlu}_{5}$ Positive allosteric modulators facilitate long-term potentiation via disinhibition mediated by $\mathrm{mGlu}_{5}$-endocannabinoid signaling. ACS Pharmacol Transl Sci 2:198-209.

Xing B, Han G, Wang MJ, Snyder MA, and Gao WJ (2018) Juvenile treatment with mGluR2/3 agonist prevents schizophrenia-like phenotypes in adult by acting through GSK3 $\beta$. Neuropharmacology 137:359-371.

Xu H, Staszewski L, Tang H, Adler E, Zoller M, and Li X (2004) Different functional roles of T1R subunits in the heteromeric taste receptors. Proc Natl Acad Sci USA 101:14258-14263.

Xu J, Zhu Y, Contractor A, and Heinemann SF (2009) mGluR5 has a critical role in inhibitory learning. $J$ Neurosci 29:3676-3684.

Xu W, Tse YC, Dobie FA, Baudry M, Craig AM, Wong TP, and Wang YT (2013) Simultaneous monitoring of presynaptic transmitter release and postsynaptic receptor trafficking reveals an enhancement of presynaptic activity in metabotropic glutamate receptor-mediated long-term depression. J Neurosci 33:5867-5877.

Xue L, Rovira X, Scholler P, Zhao H, Liu J, Pin JP, and Rondard P (2015) Major ligand-induced rearrangement of the heptahelical domain interface in a GPCR dimer. Nat Chem Biol 11:134-140.

Yamasaki T, Fujinaga M, Kawamura K, Yui J, Hatori A, Ohya T, Xie L, Wakizaka H, Yoshida Y, Fukumura T, et al. (2012) In vivo measurement of the affinity and density of metabotropic glutamate receptor subtype 1 in rat brain using 18F-FITM in small-animal PET. J Nucl Med 53:1601-1607.

Yamasue H, Aran A, and Berry-Kravis E (2019) Emerging pharmacological therapies in fragile X syndrome and autism. Curr Opin Neurol 32:635-640.

Yang JH, Mao LM, Choe ES, and Wang JQ (2017) Synaptic ERK2 phosphorylates and regulates metabotropic glutamate receptor 1 in vitro and in neurons. Mol Neurobiol 54:7156-7170.

Yang L, Mao L, Chen H, Catavsan M, Kozinn J, Arora A, Liu X, and Wang JQ (2006) A signaling mechanism from $\mathrm{G}$ alpha q-protein-coupled metabotropic glutamate receptors to gene expression: role of the c-Jun N-terminal kinase pathway. J Neurosci 26:971-980

Yin S, Noetzel MJ, Johnson KA, Zamorano R, Jalan-Sakrikar N, Gregory KJ, Conn PJ, and Niswender CM (2014) Selective actions of novel allosteric modulators reveal functional heteromers of metabotropic glutamate receptors in the CNS. J Neurosci 34:79-94.

Yin S, Zamorano R, Conn PJ, and Niswender CM (2013) Functional selectivity induced by mGlu4 receptor positive allosteric modulation and concomitant activation of Gq coupled receptors. Neuropharmacology 66:122-132.

Yohn SE, Foster DJ, Covey DP, Moehle MS, Galbraith J, Garcia-Barrantes PM, Cho $\mathrm{HP}$, Bubser M, Blobaum AL, Joffe ME, et al. (2018) Activation of the mGlu metabotropic glutamate receptor has antipsychotic-like effects and is required for efficacy of $\mathrm{M}_{4}$ muscarinic receptor allosteric modulators. Mol Psychiatry DOI: 10 1038/s41380-018-0206-2 [published ahead of print]

Yoshioka H, Sugita M, and Kinouchi H (2009) Neuroprotective effects of group II metabotropic glutamate receptor agonist DCG-IV on hippocampal neurons in transient forebrain ischemia. Neurosci Lett 461:266-270.

Young MR, Fleetwood-Walker SM, Dickinson T, Blackburn-Munro G, Sparrow H, Birch PJ, and Bountra C (1997) Behavioural and electrophysiological evidence supporting a role for group I metabotropic glutamate receptors in the mediation of nociceptive inputs to the rat spinal cord. Brain Res 777:161-169.

Young MR, Fleetwood-Walker SM, Mitchell R, and Dickinson T (1995) The involvement of metabotropic glutamate receptors and their intracellular signalling pathways in sustained nociceptive transmission in rat dorsal horn neurons. Neuropharmacology 34:1033-1041.

Youssef EA, Berry-Kravis E, Czech C, Hagerman RJ, Hessl D, Wong CY, Rabbia M Deptula D, John A, Kinch R, et al.; FragXis Study Group (2018) Effect of the mGluR5-NAM basimglurant on behavior in adolescents and adults with Fragile X Syndrome in a randomized, double-blind, placebo-controlled trial: FragXis phase 2 results. Neuropsychopharmacology 43:503-512.

Zalewska A and Wiśniewski K (1997) Behavioral activity of 1S,3R-ACPD, an agonist of metabotropic glutamate receptors. Pol J Pharmacol 49:239-248.

Zalewska-Wińska A and Wiśniewski K (2000) Behavioural activity of (S)-3,5-DHPG, a selective agonist of group I metabotropic glutamate receptors. Pharmacol Res 42: 239-245.

Zeitz C, Forster U, Neidhardt J, Feil S, Kälin S, Leifert D, Flor PJ, and Berger W (2007) Night blindness-associated mutations in the ligand-binding, cysteine-rich, and intracellular domains of the metabotropic glutamate receptor 6 abolish protein trafficking. Hum Mutat 28:771-780.

Zeitz C, Robson AG, and Audo I (2015) Congenital stationary night blindness: an analysis and update of genotype-phenotype correlations and pathogenic mechanisms. Prog Retin Eye Res 45:58-110.

Zeleznikow-Johnston AM, Renoir T, Churilov L, Li S, Burrows EL, and Hannan AJ (2018) Touchscreen testing reveals clinically relevant cognitive abnormalities in a mouse model of schizophrenia lacking metabotropic glutamate receptor 5 . Sci Rep 8:16412

Zerbib F, Bruley des Varannes S, Roman S, Tutuian R, Galmiche JP, Mion F, Tack J, Malfertheiner P, and Keywood C (2011) Randomised clinical trial: effects of monotherapy with ADX10059, a mGluR5 inhibitor, on symptoms and reflux events in patients with gastro-oesophageal reflux disease. Aliment Pharmacol Ther 33:911-921.

Zerbib F, Keywood C, and Strabach G (2010) Efficacy, tolerability and pharmacokinetics of a modified release formulation of ADX10059, a negative allosteric modulator of metabotropic glutamate receptor 5: an esophageal $\mathrm{pH}$-impedance study in healthy subjects. Neurogastroenterol Motil 22:859-865, e231.

Zhang Y, Rodriguez AL, and Conn PJ (2005) Allosteric potentiators of metabotropic glutamate receptor subtype 5 have differential effects on different signaling pathways in cortical astrocytes. J Pharmacol Exp Ther 315:1212-1219.

Zhang Z, Liu Y, Wang K, Zhu K, Zheng X, Wang L, Luan Y, Wang X, Lu H, Wu K, et al. (2019) Activation of type 4 metabotropic glutamate receptor promotes cell apoptosis and inhibits proliferation in bladder cancer. J Cell Physiol 234:2741-2755.

Zhang ZY, Sun BL, Liu JK, Yang MF, Li DW, Fang J, Zhang S, Yuan QL, and Huang SL (2015) Activation of mGluR5 attenuates microglial activation and neuronal apoptosis in early brain injury after experimental subarachnoid hemorrhage in rats. Neurochem Res 40:1121-1132.

Zheng GZ, Bhatia P, Daanen J, Kolasa T, Patel M, Latshaw S, El Kouhen OF, Chang R, Uchic ME, Miller L, et al. (2005) Structure-activity relationship of triazafluorenone derivatives as potent and selective mGluR1 antagonists. $J$ Med Chem 48:7374-7388.

Zhou F, Yao HH, Wu JY, Yang YJ, Ding JH, Zhang J, and Hu G (2006) Activation of Group II/III metabotropic glutamate receptors attenuates LPS-induced astroglial neurotoxicity via promoting glutamate uptake. J Neurosci Res 84:268-277.

Zhu CZ, Baker S, EI-Kouhen O, Lehto SG, Hollingsworth PR, Gauvin DM, Hernandez G, Zheng G, Chang R, Moreland RB, et al. (2008) Analgesic activity of metabotropic glutamate receptor 1 antagonists on spontaneous post-operative pain in rats. Eur $J$ Pharmacol 580:314-321.

Zhu PJ, Chen CJ, Mays J, Stoica L, and Costa-Mattioli M (2018) mTORC2, but not mTORC1, is required for hippocampal mGluR-LTD and associated behaviors. Nat Neurosci 21:799-802.

Zolkowska D, Kondrat-Wrobel MW, Florek-Luszczki M, and Luszczki JJ (2016) Influence of MPEP (a selective mGluR5 antagonist) on the anticonvulsant action of novel antiepileptic drugs against maximal electroshock-induced seizures in mice. Prog Neuropsychopharmacol Biol Psychiatry 65:172-178.

Zuena AR, Iacovelli L, Orlando R, Di Menna L, Casolini P, Alemà GS, Di Cicco G, Battaglia G, and Nicoletti F (2018) In vivo non-radioactive assessment of mGlu5 receptor-activated polyphosphoinositide hydrolysis in response to systemic administration of a positive allosteric modulator. Front Pharmacol 9:804.

Zussy C, Gómez-Santacana X, Rovira X, De Bundel D, Ferrazzo S, Bosch D, Asede D Malhaire F, Acher F, Giraldo J, et al. (2018) Dynamic modulation of inflammatory pain-related affective and sensory symptoms by optical control of amygdala metabotropic glutamate receptor 4. Mol Psychiatry 23:509-520. 